\title{
Molecular mechanisms of synaptic vesicle recycling with a focus on Endophilin A and Rabconnectin-3a
}

\author{
PhD Thesis \\ in partial fulfilment of the requirements \\ for the degree "Doctor of Philosophy (PhD)" \\ in the Neuroscience Program \\ at the Georg August University Göttingen, \\ Faculty of Biology
}

submitted by

SINDHUJA GOWRISANKARAN

born in

CHENNAI, INDIA

Göttingen, September 2018 
Thesis committee members:

Supervisor, reviewer: Dr. Ira Milosevic

Synaptic Vesicle Dynamics

European Neuroscience Institute

University Medical Center Göttingen

Göttingen, Germany

PhD committee member, reviewer: Prof. Dr. Reinhard Jahn

Department of Neurobiology

Max-Planck Institute for Biophysical Chemistry

Göttingen, Germany

PhD committee member: Prof. Dr. Nils Brose

Department of Molecular Neurobiology

Max Planck Institute for Experimental Medicine

Göttingen, Germany

Extended committee members:

Prof. Dr. Tobias Moser

University Medical Center

Institute for Auditory Neuroscience \& InnerEarLab

Göttingen, Germany

Nuno Raimundo, PhD

Institute of Cellular Biochemistry

University Medical Center

Göttingen, Germany

Dr. Christian Vogl

Institute for Auditory Neuroscience \& InnerEarLab

University Medical Center

Göttingen, Germany

Date of the oral examination: 06.11.2018 


\section{Table of Contents}

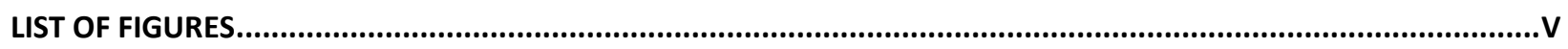

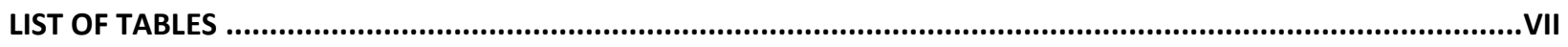

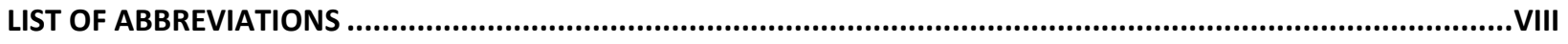

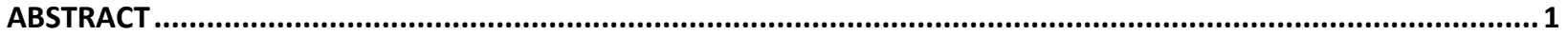

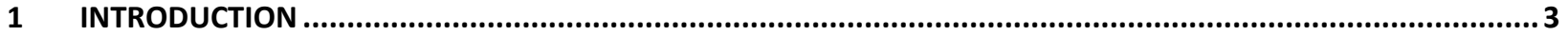

$1.1 \quad$ NEURONAL COMMUNICATION THROUGH SYNAPTIC TRANSMISSION .................................................................

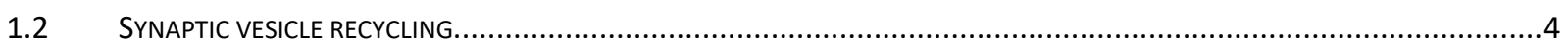

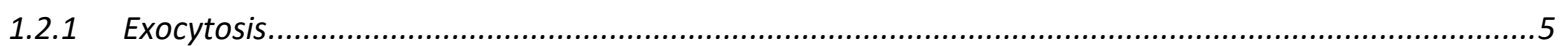

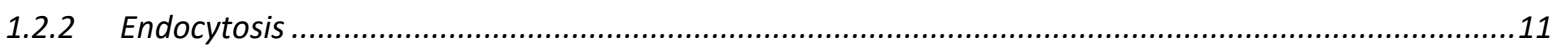

1.2.3 Vesicle acidification and neurotransmitter uptake .......................................................................20

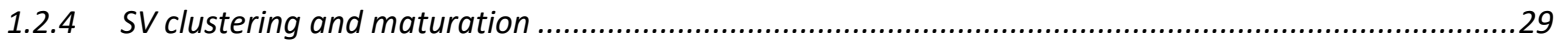

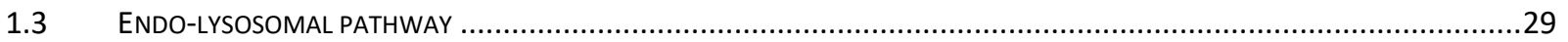

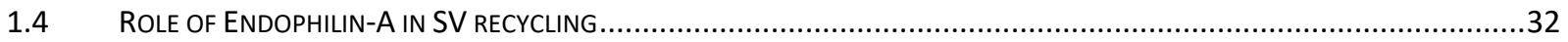

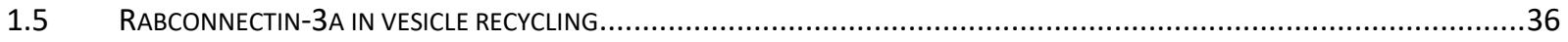

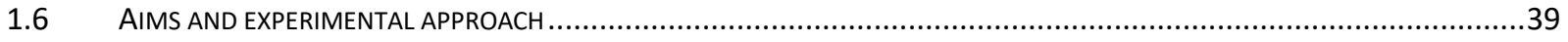

1.6.1 Chromaffin cell as a model system to study regulated exocytosis................................................39

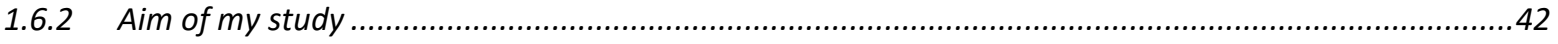

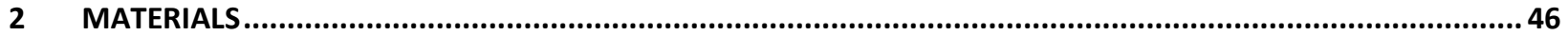

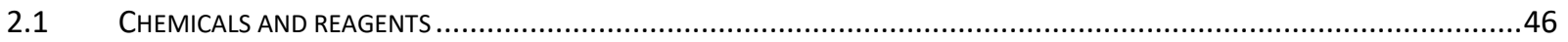

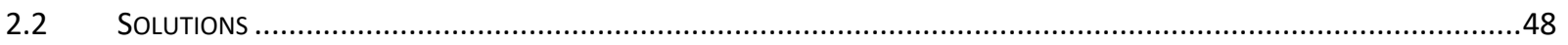

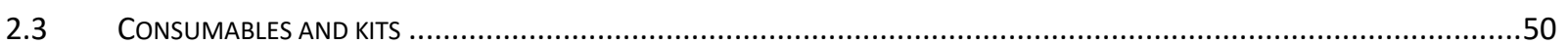

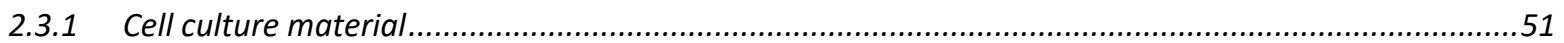

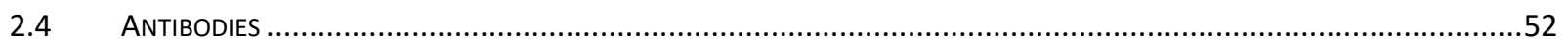

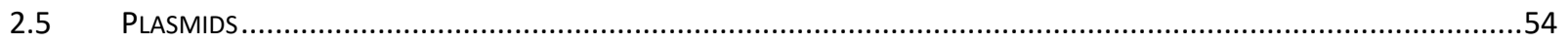

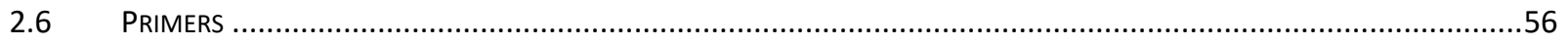

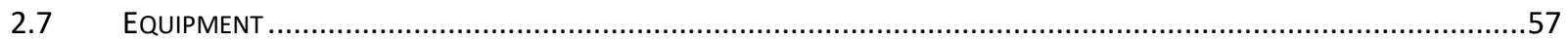

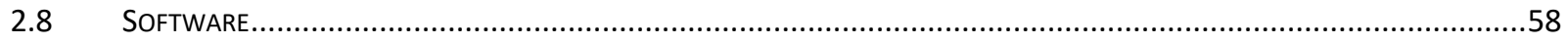

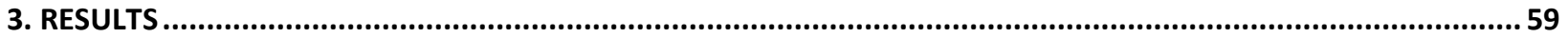


3.1 ENDOPHILIN-A CONTROLS RECRUITMENT, PRIMING AND FUSION OF NEUROSECRETORY VESICLES

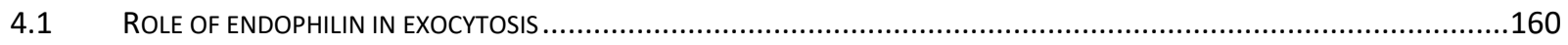

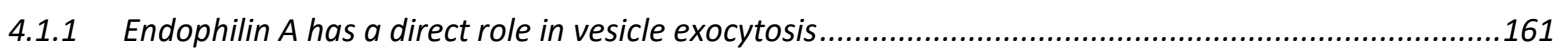

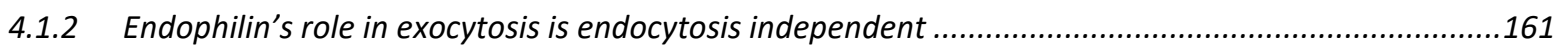

4.1.3 Mechanism of endophilin's action in exocytosis: BAR domain vs SH3 domain .................................162

4.1.4 Role of endophilin and dynamin in neurosecretory vesicle fusion ...............................................163

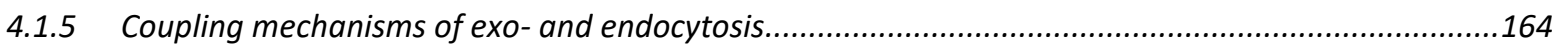

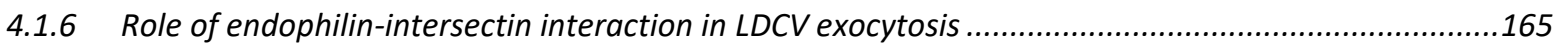

4.2 RBCN-3A IS A PUTATIVE V-ATPASE REGULATOR THAT LINKS ENDOCYTOSIS, ACIDIFICATION AND SV MATURATION .............166

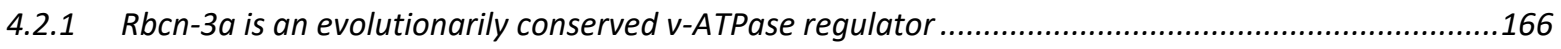

4.2.2 Links between endocytosis and vesicle acidification...............................................................167

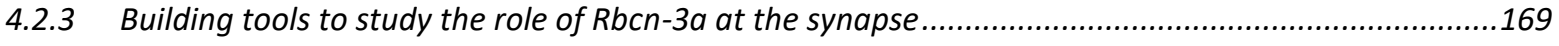

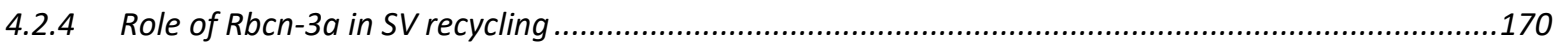

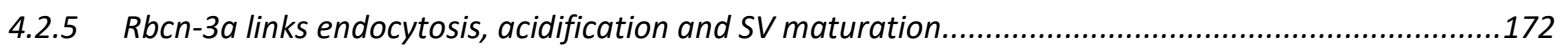

4.2.6 V-ATPase and its regulator at the cross road between cellular trafficking and metabolic pathways .175

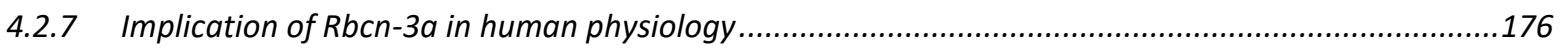

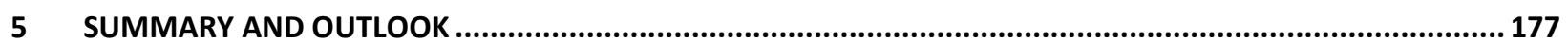

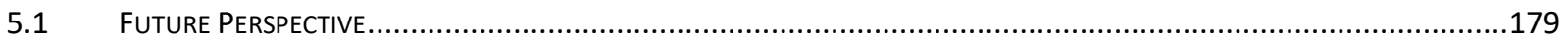

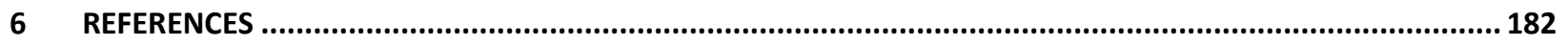

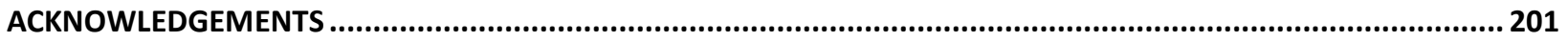




\section{List of figures}

Figure 1 Neurotransmission at the chemical synapse ......................................... 4

Figure 2 SV recycling and architecture of site for vesicle exocytosis.......................... 6

Figure 3 Pathways of endocytosis at the neuronal synapse ................................. 13

Figure 4 Model of clathrin mediated endocytosis.......................................... 16

Figure 5 Structure of V-ATPase and its regulation during SV recycling ....................... 23

Figure 6 Cellular membrane trafficking pathway........................................... 31

Figure 7 Structure and function of Endophilin A in SV recycling.............................. 35

Figure 8 Molecular interactions of Rabconnectin-3a...................................... 37

Figure 9 Regulated exocytosis measurements in adrenal chromaffin cells.................. 41

\section{Chapter 3.1}

Figure 1. Endophilins are present in adrenal chromaffin cells and enriched at the

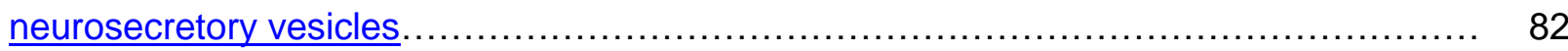

Figure 2. Lack of endophilins reduces exocytosis in mouse chromaffin cells................. 84

Figure 3. Expression of endophilin 1 and endophilin 2 rescued exocytosis in endophilin

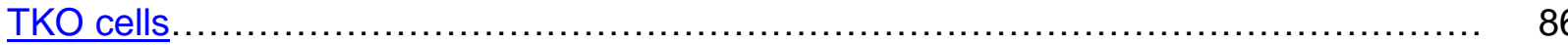

Figure 4. Number and size of LDCVs were not altered in endophilin TKO chromaffin cells, but less LDCVs was found in the plasma membrane proximity ............................ 88

Figure 5. Exocytic machinery is unaltered in the chromaffin cells without endophilin........ 90

Figure 6. Endocytic defects in the absence of endophilin in chromaffin cells................. 92

Figure 7. Endophilin 1 BAR and endophilin 2 BAR-domains are not sufficient to mediate exocytic release from chromaffin cells.

Figure 8. Endophilin's role in exocytosis is mediated, at least in part, through its interaction with intersectin

Figure S1. Endophilin on isolated LDCVs and re-distribution of endophilin upon chromaffin cell stimulation. 105

Figure S2. Double-log plot shows correlation between the lower time constant of the RRP and the lower pool size of the RRP

Figure S3. Expression of endophilin 1 and endophilin 2 rescued exocytosis in endophilin TKO cells ( $2^{\text {nd }}$ stimulus) 
Figure S4. Levels of key exocytic proteins were unaltered in mouse adrenal gland homogenates.

Figure S5. Characterization of uptake of mCling-Atto647 and cholera toxin-B-A594 endocyctic assays in adrenal chromaffin cells. 109

Figure S6. Levels of intersectin-1 protein were not altered in endophilin TKO cells. 110

Figure S7. F-actin network is altered in the endophilin TKO cells. 111

Figure S8. Model of endophilin and intersectin's role in exocytosis. 112

\section{Chapter 3.2}

Figure 1. SV-associated protein Rbcn-3a is found on CCVs and localized at CCPs together with v-ATPase. 133

Figure 2. Rbcn-3a KO neurons in culture formed synapses, but the total levels of integral SV proteins were reduced. 135

Figure 3. Neurons lacking Rbcn-3a had altered synapse morphology and impaired synaptic transmission......

Figure 4. SV recycling was impaired due to a delayed post stimulus recovery in Rbcn-3a KOs. 139

Figure 5. Compensatory endocytosis was not changed in Rbcn-3a KO neurons 141

Figure 6. SV membrane potential was reduced in the absence of Rbcn-3a. 142

Figure 7. Loss of Rbcn-3a reduced the Rab3 stability and recruitment on SVs at the synapses. 143

Figure 8. Rbcn-3a KO neurons accumulated lysosome-like structures 144

Figure S1. Rbcn-3a and v-ATPase are localized to clathrin coated pits (CCP) in fibroblasts

Figure S2. Rbcn-3a KO strategy and validation in neurons. 155

Figure S3. Synaptic vesicles isolated by immuno-isolation were functionally active. 156

Figure 10 Hypothetical model proposed to explain the role of Rbcn-3a in SV recycling pathway. 


\section{List of tables}

Table 1 - List of chemicals, their supplier and catalog number 46

Table 2 - List of buffers and solutions used in this study 48

Table 3 - Kits and consumable products used in this study 50

Table 4 - List of cell culture material 51

Table 5 - Primary antibodies used for immunohistochemistry 52

Table 6 - Secondary antibodies used for immunohistochemistry 53

Table 7 - Primary antibodies used for western blot analysis 53

Table 8 - Secondary antibodies used for western blot analysis $\quad 54$

Table 9 - Plasmid used in the study 55

Table 10 - Primers used for genotyping samples $\quad 56$

Table 11 - Primers used for quantitative PCR 56

Table 12 - List of equipments used in this study 57

Table 13 - List of software programs 58 


\section{List of abbreviations}

\begin{tabular}{ll}
${ }^{\circ} \mathrm{C}$ & degree celcius \\
aa & amino acid \\
AP2 & adaptor protein 2 \\
ATP & adenosine 5'- triphosphate \\
BAR domain & (Bin/Amphiphysin/RVS)-domain \\
BSA & Bovine serum albumin \\
CCP & clathrin coated pits \\
CCV & clathrin coated vesicle \\
CIE & clathrin-independent endocytosis \\
CME & clathrin-mediated endocytosis \\
CMV & human cytomegalovirus \\
CNS & central nervous system \\
DIV & days-in-vitro \\
DMEM & Dulbecco's modified eagle's medium \\
DMXL2 & DMX-like protein 2 \\
DNA & Deoxyribonucleic acid \\
EE & early endosomes \\
EEA1 & Early endosomal antigen 1 \\
eGFP & enhanced green fluorescent protein \\
EPSC & excitatory postsynaptic current \\
FBS & Fetal bovine serum \\
GABA & Y-Amino butyric Acid \\
GAP & GTPase-activating protein \\
GDI & Rab GDP dissociation inhibitor \\
GDP & guanosine diphosphate \\
GEF & GDP/GTP exchange protein \\
GFP & Green fluorescent protein \\
GnRH & gonadotropin-releasing hormone \\
GTP & guanosine-5'-triphosphate \\
HBSS & Hank's Balanced Salt solution \\
HEPES & 4-(2-hydroxyethyl)-1-piperazineethanesulfonic acid \\
IPSC & inhibitory postsynaptic current \\
ITSN & intersectin \\
kDa & kilo daltons \\
LAMP1 & lysosomal-associated membrane protein 1 \\
LDCV & large dense core vesicles \\
LE & late endosome \\
& \\
\hline
\end{tabular}




\begin{tabular}{|c|c|}
\hline MAP2 & Microtubule-associated protein 2 \\
\hline mTORC1 & mammalian/mechanistic Target of Rapamycin \\
\hline NT & Neurotransmitter \\
\hline PBS & phosphate buffered saline \\
\hline PEPNS & polyendocrine-polyneuropathy syndrome \\
\hline PFA & paraformaldehyde \\
\hline $\mathrm{PI}(4,5) \mathrm{P} 2$ & Phosphatidylinositol 4,5-bisphosphate \\
\hline PI3P & phosphatidylinositol 3-phosphate \\
\hline PLL & Poly-L-Lysine \\
\hline PM & plasma membrane \\
\hline PMSF & Phenylmethylsulfonyl fluoride \\
\hline RAVE & regulator of $\mathrm{H}^{+}$-ATPase of vacuolar and endosomal membrane \\
\hline Rbcn-3a & rabconnectin-3a \\
\hline Rbcn-3b & rabconnectin-3b \\
\hline RFP & red fluorescent protein \\
\hline RIM & Rab3-interacting molecule \\
\hline RPM & revolutions per minute \\
\hline $\mathrm{RRP}$ & readily releasable pool \\
\hline RT & room temperature \\
\hline SDS-PAGE & Sodium dodecyl sulfate polyacrylamide gel electrophoresis \\
\hline $\mathrm{SH} 3$ & Src-homology 3 \\
\hline SNARE & Soluble N-ethylmaleimide-sensitive factor attachment protein receptors \\
\hline SRP & slowly releasable pool \\
\hline SV & synaptic vesicle \\
\hline SVs & synaptic vesicles \\
\hline TBS-T & Tris-Buffered Saline and Tween 20 \\
\hline TIRF & total internal reflection fluorescence microscope \\
\hline TKO & triple knock-out \\
\hline v-ATPase & vacuolar-type $\mathrm{H}^{+}$-adenosine 5 '-triphosphatase \\
\hline vGLUT & vesicular glutamate transporter \\
\hline WD & Trp-Asp (W-D) dipeptide containing domain \\
\hline
\end{tabular}




\section{Abstract}

Synaptic vesicle (SV) recycling at the synapse is fundamental to neurotransmission and it sustains unimpaired neuronal communication. It is a complex cell biological process, where the molecular steps are finely coordinated and intensively studied in the last three decades. The basic principles and the regulatory mechanisms underlying key steps like exocytosis and endocytosis have been broadly understood. Yet, there are several missing links in SV recycling process. Some of the open questions that are not well understood include the mechanism of how exocytosis is coupled with endocytic retrieval, how a newly formed vesicle (through endocytosis) is transformed into a fusion-competent vesicle for the next round of events, and how the SV recycling process is regulated. Several major molecular players have been identified and their roles characterized in specific steps of the recycling process. But, how specific proteins or factors are made available at the right time for the precise step in the process is not fully understood.

In this study, we explore some of the missing links in the SV recycling process. The first study reports a new role for an endocytic protein called endophilin-A (henceforth endophilin) in exocytosis and potential link to the coupling mechanism between exocytosis and endocytosis. In the second study, we characterized the role of a protein called rabconnectin-3a in SV recycling process and how it potentially acts as a link between several steps: endocytosis, vesicle acidification and maturation of newly formed vesicles.

Endophilin is a well-studied endocytic adaptor protein. We found a direct role for endophilin in large dense-core vesicle (LDCV) exocytosis, independent of its role in endocytosis, using the neurosecretory adrenal chromaffin cell and mouse model lacking all three endophilins. The lack of endophilin affects the vesicle recruitment, priming and fusion during the exocytic process. These exocytic defects could be rescued by both endophilin A1 and endophilin A2. The reduced exocytosis was not due to a reduction in the number of vesicles, since the LDCV number per cell, or cell area, was unaltered in the absence of endophilin. The absence of endophilin did not majorly affect protein and membrane recycling by endocytic mechanisms. Further, none of the major exocytic or endocytic factors were affected in its absence. SH3 domain of endophilin was important for its function in exocytosis. The role of endophilin in vesicle recruitment and release, at least in part was mediated through the interaction with a scaffolding protein, called intersectin. 
This study offers an example of how proteins can assist several steps in vesicle recycling process and how protein-protein interactions help to keep endocytic proteins compartmentalized, to make them available at the right moment for their action in the vesicle cycle.

In the second study, we focused on how the process of endocytosis is linked to vesicle acidification and refilling of neurotransmitters (NT) to form a fusion-competent vesicle. Rabconnectin-3a (Rbcn-3a) is a putative regulator of v-ATPase, the proton pump on the vesicle that is responsible for acidification process. We characterized the role of Rbcn-3a in SV recycling in neurons. Rbcn-3a is present on both synaptic vesicles, as well as clathrin-coated vesicles. Although the mice without Rbcn-3a die in the early embryonic stage, neurons developed normally and formed synapses. Yet, in the absence of Rbcn-3a, the number of SVs was reduced at synapses and neuronal activity was impaired. Presynaptic activity monitored using pHluorin tagged to vGLUT1 showed that the recovery after stimulation is delayed in the absence of Rbcn3a. This delay was not due to a fault in endocytic retrieval, but due to a defective acidification of the vesicles. As a consequence of defective recycling, recruitment of Rab3 on SVs was reduced and its stability on the vesicles was impaired in Rbcn-3a knock-out neurons. Neurons also accumulated lysosome-like structure, suggesting a role for Rbcn-3a in membrane trafficking pathways in neurons. This study reveals a role for Rbcn-3a in regulation of vesicle acidification and how it links endocytosis to acidification and recruitment of peripheral proteins for vesicle maturation process during recycling.

The findings reported in my doctoral thesis work offer some information on the missing links in the vesicle recycling pathway. It also reinstates how the recycling process is tightly coordinated: the molecular players can participate in more than one step of the recycling process and link them. 


\section{Introduction}

\subsection{Neuronal communication through synaptic transmission}

Communication between neurons forms the basis of thinking, motor control, cognitive processing and memory among other functions. In order to comprehend such complex human behavior, we need to understand the molecular mechanisms occurring in the fundamental building blocks of the brain. Brain function depends on diverse cell types, especially, neurons and glia. Neurons form the structural and functional unit and are electrically excitable cells of the brain (Kandel et al., 2012). Glial cells, which outnumber the neurons in their count, provide structural support to the neurons as well as help in maintaining homeostasis (Marina et al., 2018). A neuron has a cell body or soma and processes (dendrite and axon). The soma or perikaryon contains the nucleus and the organelles embedded in the cytoplasm. Dendrites are highly branched structures, and along with the soma, receive input from the other cells. Axon is a single specialized projection of the neuron, involved in the flow of information from the soma to other neurons (Brodal, 2010) (Figure 1a).

The axonal terminal forms a structure called synapse, which are juxtapositions formed between an axon of one neuron (presynaptic cell) and the soma/dendrite of another neuron (postsynaptic cell). The intercellular space at the contact point of a synapse is called synaptic cleft (Brodal, 2010) (Figure 1a and b). Synapses facilitate fast signal transduction through chemical or electrical neurotransmission. In electrical synapses, the nerve impulses travel rapidly along gap junctions, where voltage changes in one cell can induce a voltage change in the opposing cell at the junction (Kandel et al., 2012). Importantly, synapses not only help in information propagation, but also transform it.

Neurotransmission at chemical synapses involves the release of chemical signals called neurotransmitters (NTs) by the presynaptic neuron upon receiving an electro-chemical excitation called action potential. NTs are stored inside small, spherical, membrane delimited structures called synaptic vesicles (SVs) at the bouton (Figure 1b). NTs are released in discrete amounts called quanta. The released NTs in the cleft further bind to their receptors on the membrane of the post synaptic cell and inflict an effect on neuron by two possible ways. NT binding to the receptor can open a voltage gated ion channel (ionotropic receptor), leading to change in 
membrane permeability to some ions. Alternatively, binding of NT to the receptor (metabotropic receptors) can initiate a second messenger cascade, which can induce changes to the postsynaptic neuron. Both of these events trigger changes in the net movement of charged species in postsynaptic neuron, which further propagates the signal.

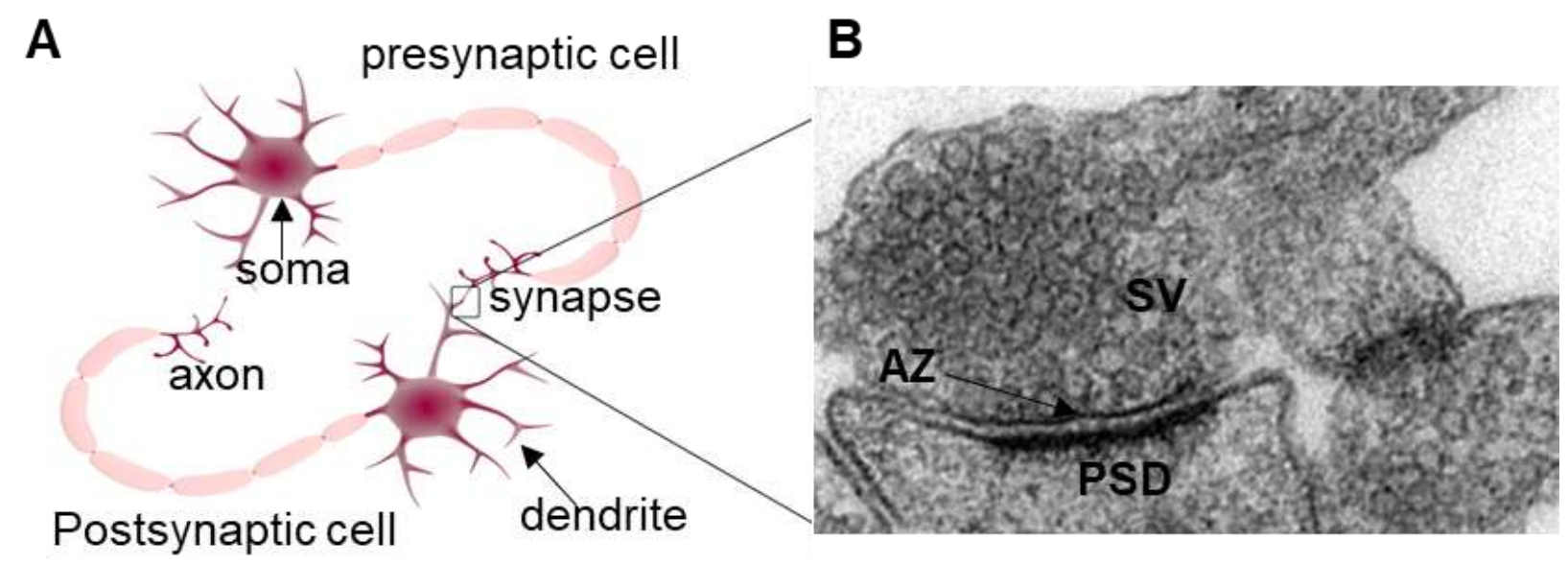

Figure 1 Neurotransmission at the chemical synapse

(A) Illustration of neurons with cell body (soma), dendritic branches and a specialized projection that allows flow of information (axon). A synapse (box) formed by an axon of one neuron (presynaptic cell) and dendrite of another neurons (postsynaptic cell) is depicted here. (B) Ultrastructure of a synapse obtained using electron microscope. Numerous synaptic vesicles (SV) are present at the bouton, which release NT at designated sites called the active zone (AZ). The released NT bind to receptor on the postsynaptic cell organized at specialized zones called postsynaptic density (PSD) (exemplary EM image for illustration).

\subsection{Synaptic vesicle recycling}

At the presynaptic terminal, a sequence of events leading to fusion of vesicles is triggered upon receiving an action potential. SVs are recruited to the active zone, an electron-dense region at the plasma membrane (PM, sites of fusion at the central synapse), upon influx of calcium due to opening of voltage-gated calcium channels. Vesicles undergo SNARE dependent fusion process, called exocytosis (Figure 2) (reviewed in (Südhof and Rizo, 2011) and (Jahn and Fasshauer, 2012). SVs accumulate at the synaptic terminals, where they store and secrete different types of NTs. The demand for SVs during exocytosis at a synapse over any given time exceeds the supply of vesicles from the cell soma. Nerve terminals have developed efficient ways to recycle the 
excess SV membrane and protein added during exocytosis to PM by the process of endocytosis (Figure 3) (Saheki and De Camilli, 2012). Endocytosis helps to maintain the total surface area a constant and support repeated rounds of fusion, apart from sustaining high rate of transmission. New SVs are formed from the internalized material; undergo further steps like filling with NT, recruitment of peripheral proteins to become competent to participate in subsequent cycles of fusion (Purves et al., 2018). This complete cycle of events, called SV recycling (Heuser and Reese, 1973), is crucial to sustain uninterrupted communication during neuronal activity. In the next section, the various steps in SV recycling are outlined.

\subsubsection{Exocytosis}

Eukaryotic cells have characteristic fusion of membrane-delimited organelle, which forms the basis of several key processes including synaptic transmission and immunity. Regulated exocytosis is fundamental to neurotransmission and involves series of protein-protein interaction, resulting in the release of vesicular content (NT and secretory hormones). Many key proteins central to neuronal exocytosis and neuroendocrine secretory system have been identified. They orchestrate steps, including mobilization of vesicle to the release site, docking and priming of vesicles, $\mathrm{Ca}^{2+}$ influx triggered vesicle fusion, finally leading to the release of vesicular content by fusion with the target PM (Figure 2) (reviewed in (Südhof and Rizo, 2011) and (Jahn and Fasshauer, 2012)).

The functions of the main molecular machines have been elucidated in the last three decades using advanced imaging, electrophysiological measurements, biochemical and biophysical methods (Jahn and Fasshauer, 2012). Additionally, release properties like number of docked and primed vesicles, kinetics of fusion process, release probability and calcium dependence have been studied in depth using model systems like Calyx of held (glutamatergic central synapse involved in the auditory pathway), adrenal chromaffin cell (catecholamine release), and hippocampal synapses (NT release) (Neher, 2006), among other in-vitro systems and model organisms. Although the fundamental mechanism of operation and release is similar between different synapses, they differ not only in the vesicular content, but also in basic synaptic properties and computational function (Südhof, 2012). 

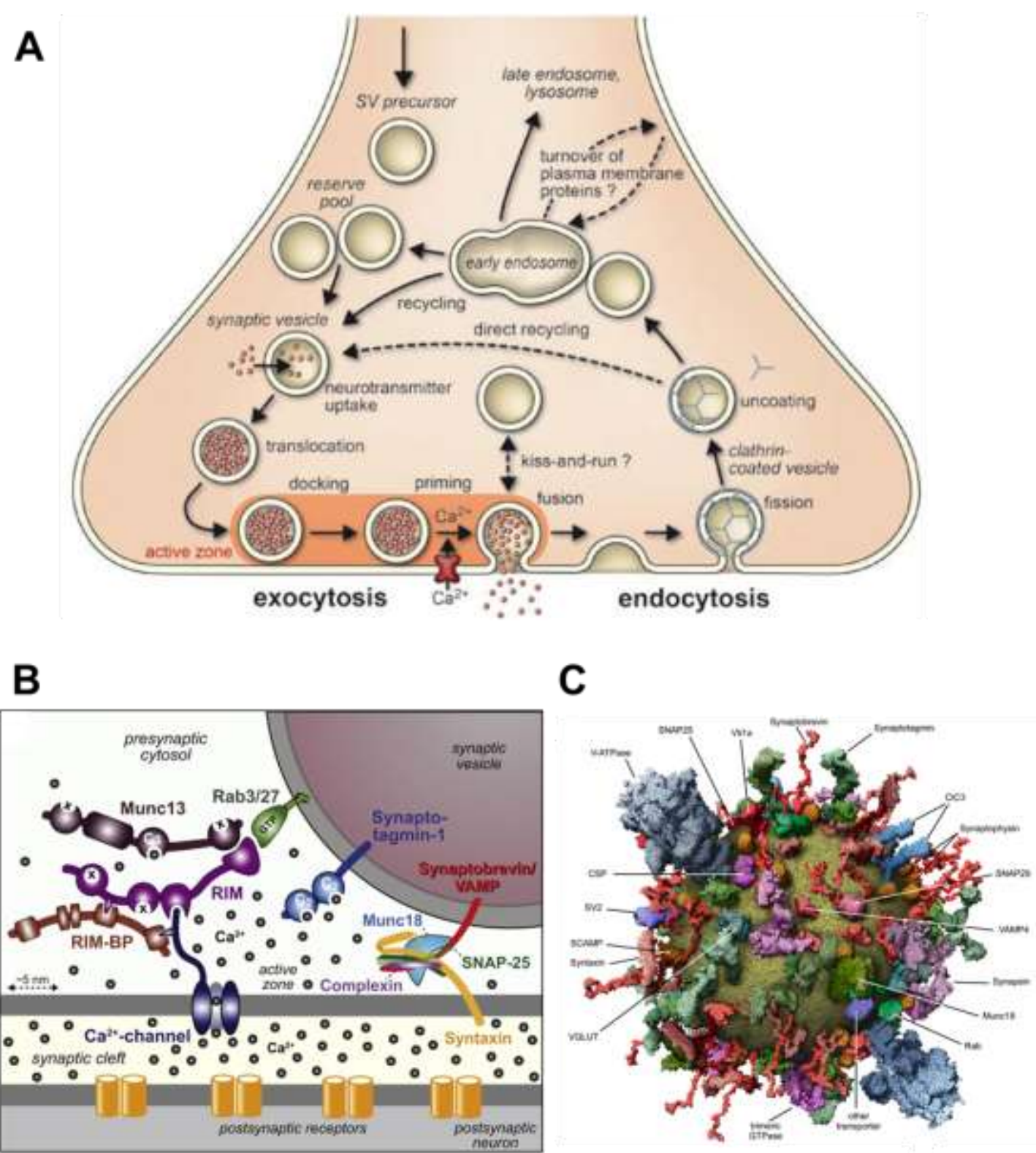

\section{Figure $2 \mathrm{SV}$ recycling and architecture of site for vesicle exocytosis}

(A) Neurotransmission is mediated by the release of NT stored inside organelles called synaptic vesicles (SVs) by the process of exocytosis. It involves a sequence of steps including mobilization of NT filled SV to specialized sites for release called the active zone, where the vesicles are docked, followed by the activation of release machinery by priming and finally undergoes calcium-triggered exocytosis. The excessive membrane and proteins are retrieved by endocytosis; new vesicles are formed subsequently and filled with NT again to participate in the next cycle of fusion events (adapted from Jahn and Fasshauer, 2012). (B) Architecture of the release sites showing the protein machinery that orchestrates the regulated 
exocytosis process. Proteins from different families like Rabs, SM proteins and CATCHR orchestrate steps leading to the fusion mediated by the SNARE proteins (adapted from Südhof, 2013). (C) Molecular model of an average synaptic vesicle showing the crowded protein density on them (adapted from (Takamori et al., 2006)).

In the following section, I will briefly summarize the key steps in the exocytosis process and describe the molecular machines involved in these steps, with a focus on central synapses and neurosecretory model system (adrenal chromaffin cell).

\subsubsection{Mobilization of vesicles to the release sites}

SVs are $\sim 40 \mathrm{~nm}$ in diameter, uniformly sized organelles, primarily involved in neurotransmitter release (Figure 2). Presynaptic terminal in the brain typically contains hundreds of vesicles, organized in functionally distinct pools called vesicle clusters or vesicle pools (Rizzoli and Betz, 2005). Vesicles are held together to form pools at a resting state by a protein-protein meshwork involving synapsins, intersectin, Rabs and $\mathrm{SH} 3$ domain containing proteins (Pechstein and Shupliakov, 2010; Milovanovic and De Camilli, 2017). The vesicles are mobilized between the different pools within a synapse, as well as exchanged with the neighboring synapses, to facilitate proper transmission depending on the need during activity.

Majority of the vesicles at a terminal do not participate in the recycling process actively. Recycling vesicles support release upon moderate stimulation and include a small fraction of vesicles. The most active among these vesicle form a pool called readily releasable pool (RRP) and available immediately for release process (Rosenmund and Stevens, 1996). At resting state, only a few vesicles (belonging to the RRP) are in the state that can undergo fusion instantly (Alabi and Tsien, 2012). Near the PM of the terminal, an electron-dense cytomatrix formed by multi-domain proteins of the active zone organize the site of release where SV are recruited to undergo fusion. The core proteins that form this protein complex include RIM (Rab3 interacting molecule), Munc13, ELKS, CAST, Liprin, Bassoon and Piccolo. Active zone proteins couple the vesicles in release-ready state with calcium channels in the PM. They are also responsible for precise localization of preand postsynaptic specialization and mediate short- and long-term presynaptic plasticity. The first step in calcium $\left(\mathrm{Ca}^{2+}\right)$ triggered vesicle exocytosis involves mobilization of vesicle to the active zone and is influenced by the active zone proteins (reviewed in Südhof, 2012). For example, RRP 
reloading is accelerated during activity by a scaffolding protein, Bassoon (Hallermann et al., 2010).

Unlike neuronal synapse, neurosecretory chromaffin cells lack special active zone morphologically and the vesicle release is polarized in the adrenal medulla (not in cell culture). Protein clusters (syntaxin-1, calcium channel) on the PM of cultured chromaffin cells were found to assist in organizing release of certain LDCVs (Lopez-Font et al., 2010). A pool of mature LDCVs localized in the close proximity of PM is involved in exocytic release. LDCVs are kept in a meshwork of actin filaments. Increase of $\mathrm{Ca}^{2+}$ upon stimulation leads to shift in depolymerizationrepolymerization balance of actin filaments and helps to mobilize the LDCVs closer to PM as well as make the regulatory molecules for release available (Gil et al., 2000). This step in chromaffin cell model is distinct from the SV at central synapses, wherein actin network has a scaffolding function towards regulatory proteins (Sankaranarayanan et al., 2003). In Chapter 3.1, our findings contribute to further understanding of this step, where we attribute a role for endophilin (likely through its interaction with intersectin) in LDCV mobilization for release.

\subsubsection{Preparation of vesicle for the fusion process}

Vesicles mobilized to the release site undergo regulated fusion upon activation of the fusion machinery. Some vesicles of the readily releasable pool have their membrane in contact with the $\mathrm{PM}$ at the free release sites and are morphologically defined as docked vesicles (Verhage and Sørensen, 2008). Vesicles are further made fusion competent by a process called priming that involves complex set of reversible interactions leading to the activation of release machinery. Both these steps are controlled by multiple protein interactions and it is difficult to assign any particular protein to only one of these steps (Jahn and Fasshauer, 2012). The main proteins mediating regulated exocytosis belong to the conserved protein families like SNAREs, SM (Sec1/Munc18like) proteins, Rabs and tethering factors called CATCHR (complex associated with tethering containing helical rods) (reviewed in Südhof and Rizo, 2011; Jahn and Fasshauer, 2012).

SMproteins are evolutionarily conserved players of vesicular trafficking besides the SNARE (soluble N-ethylmaleimide-sensitive factor (NSF) attachment protein receptors) proteins (Südhof and Rothman, 2009). Munc18 belongs to SM-protein family and is involved in SNARE-mediated 
vesicle fusion (Hata et al., 1993). Munc18 binds syntaxin-1 in closed configuration and controls SNARE complex formation (Dulubova et al., 1999). Complete loss of Munc18 leads to a complete block of exocytosis (Verhage et al., 2000), suggesting a positive regulatory role apart from preventing futile SNARE complex formation during trafficking to the terminal and at resting state.

Active zone proteins like Munc13 and RIM are also involved in docking and priming of SVs (Brose et al., 1995; Wang and Südhof, 2003). The binding of Munc13 and RIM is considered to be important for the priming step (Betz et al., 2001). RIM1, among other active zone proteins help to anchor the vesicle by binding to Rab3 and Rab27 (Dulubova et al., 2005), as well as to synaptotagmin-1 on the vesicle (Dulubova et al., 2005; Wang et al., 1997), and finally tethers the docked/primed vesicle with $\mathrm{Ca}^{2+}$ channels (Coppola et al., 2001; Kaeser et al., 2011) (Figure 2B) (reviewed in (Südhof and Rizo, 2011)). Munc13 has a catalytic Mun domain, which is important for priming process. Munc13 can bind syntaxin-1, SNARE complex and Munc18 and the consequence of these weak, yet important interactions could be implicated in partially opening syntaxin-1 from closed state in Munc18/syntaxin-1 dimers (Wojcik and Brose, 2007). Absence of Munc13 leads to a complete block of NT release and suggest that the protein is pivotal for neuronal exocytosis (Augustin et al., 1999). Another Mun domain containing protein involved in the priming process is CAPS, which additionally has a PH (pleckstrin homology) domain. CAPS protein is important for the priming process in neuroendocrine cells (James and Martin, 2013). It can also bind SNARE complex and open syntaxin-1 from its closed configuration, similar to that of Munc13 (Khodthong et al., 2011). Although they are both involved in opening of syntaxin-1 to a more open configuration to facilitate fusion complex formation, they contain additional domains (PH domain in CAPS versus $\mathrm{C} 1$-and $\mathrm{C} 2$ domain in Munc13) which could define their other regulatory roles (Südhof and Rizo, 2011).

\subsubsection{Calcium triggering and SNARE mediated fusion}

SNARE proteins are central components for the fusion process (Jahn et al., 2003). An alpha helix bundle is formed by a Qa, Qb, Qc and R SNARE protein to mediate fusion of the vesicle with the target membrane (Kloepper et al., 2007; Stein et al., 2009). SNARE proteins alone are able to complete fusion process in-vitro (Weber et al., 1998). However, the process is complex in-vivo 
and regulatory proteins orchestrate/regulate the SNARE mediated fusion process (Jahn and Fasshauer, 2012).

Exocytosis at the neuronal synapse is mediated by syntaxin-1 and SNAP-25 on the target PM and the vesicular SNARE protein synaptobrevin/VAMP2 (Söllner et al., 1993). Syntaxin-1 (Qa SNARE) is attached to the PM and can form clusters on the PM (Lopez-Font et al., 2010). It has a C-terminal transmembrane domain, SNARE motif, $\mathrm{H}_{\mathrm{abc}}$ domain and $\mathrm{N}$-peptide. $\mathrm{H}_{\mathrm{abc}}$ domain can bind the SNARE motif as well as with Munc18. This keeps syntaxin-1 in closed configuration until fusion process is ready to occur. A switch to an active open conformation drives the trans-SNARE complex formation for fusion (Misura et al., 2000). SNAP-25 (Qb, c) has two SNARE motifs and can attach to the PM through the linker domain via plamitoylation (Veit et al., 1996). Two isoforms of SNAP-25 is found in mammals, SNAP-25a is predominantly found in neuroendocrine cells and is important for embryonic development, while more efficient SNAP-25b is prominent in adulthood (Sørensen et al., 2003). SNAP-25 interacts with syntaxin-1 and forms the acceptor complex to bind the vesicular R-SNARE protein synaptobrevin 2/VAMP2 (Blasi et al., 1993a, 1993b; Söllner et al., 1993). $\mathrm{Ca}^{2+}$ binding to synaptotagmin triggers the completion of partially assembled SNARE complex zippering reaction that generates the force needed to pull SV and presynaptic membranes together or fusion can happen spontaneously (Jahn and Fasshauer, 2012).

SNARE mediated fusion is regulated SM and CATCHR family of proteins. Both Munc13 and 18 play a pivotal role in accurate trans-SNARE complex formation and implicated in all fusion events. The unique property of calcium triggered exocytosis can be attributed to specialized regulatory proteins like synaptotagmins and complexins (Südhof and Rizo, 2011). Synaptotagmin-1 (Syt1) is a vesicular protein with a single trans-membrane domain that anchors the protein to the membrane and has two characteristic C2 domains (C2A and C2B). Synaptotagmin 1 and 2 are the major calcium sensors for fast-triggered fusion (Geppert et al., 1991, 1994; Perin et al., 1990). The calcium affinity of $\mathrm{C} 2$ domain increases upon binding to acid phospholipid membranes. In the presence of calcium, Syt1 binds to membrane enriched in $\mathrm{PI}(4,5) \mathrm{P}_{2}$ (Chapman, 2008). The binding of calcium to $\mathrm{C} 2$ domain possibly acts as a switch to trigger the fast-fusion process. While Syt1 and 2 are involved in fast synchronous release, other synaptotagmins like 3, 6 and 7 are implicated in the slow, asynchronous release at lower $\mathrm{Ca}^{2+}$ concentration (reviewed in Südhof, 2012). Complexins are a family of small cytosolic proteins, which are not highly structured 
(McMahon et al., 1995). They bind to the SNARE complex via a central helix and this interaction is essential to exert its function in the fusion process (Glick and Rothman, 1987). Complexins have a dual inhibitory and stimulatory role in the fusion process. They act as a clamp to prevent the premature release of primed vesicles, as well as exert a positive regulatory effect by facilitating the fusion process. Calcium binding to synaptotagmin can release the clamp by complexin, thereby completing the fusion process (reviewed in (Trimbuch and Rosenmund, 2016).

After $\mathrm{Ca}^{2+}$ triggered fusion and release of the vesicular content, SNARE complex is still present on the PM in the cis-SNARE configuration. Disassembly of the complex and recycling of the SNARE proteins is mediated by NSF (AAA+ protein) and its co-factor a-SNAP (Clary et al., 1990; Littleton et al., 2001; Söllner et al., 1993). SNAREs and other proteins are retrieved from the PM to continue synaptic vesicle cycle and new vesicles are formed subsequently, which can support another round of exocytosis.

\subsubsection{Endocytosis}

Endocytosis is a fundamental process to internalize macromolecules and proteins such as channels and receptors, while some virus and bacterial toxins exploit this pathway to gain access into the cell. Internalized receptor from the cell surface is either trafficked back to PM to sustain signaling or to lysosome for degradation. Nerve terminals have developed efficient ways to remove excessive membrane and proteins added during exocytosis to continue the process of SV recycling and sustain high rate of transmission (Heuser and Reese, 1973; Saheki and De Camilli, 2012). After SV fusion and release of NT, the process of endocytosis from the PM (Figure 3) retrieves the membrane as well as the components of SVs. Compensatory endocytosis ensures that the total membrane surface area is maintained during activity and SV are locally regenerated with high efficiency and fidelity. It is widely accepted that endocytosis happens in the near vicinity of the active zone or release sites, called periactive zone. Apart from retrieving components of the vesicles, site clearance by endocytosis may be important to clear the release sites to make them free for the next fusion events (Neher, 2010). This suggest for a possible mechanism to link exo- and endocytosis, with the scaffolding proteins at the active and periactive zone linking vesicle fusion with release site clearance by endocytosis (reviewed in Haucke et al., 
2011). It is not yet understood if the recently added vesicular proteins stay in a cluster or diffuse in the PM before being recycled.

SVs are homogenous in size with unique protein composition and this warrants endocytic machinery to precisely fission and shape them during recycling. The process of endocytosis is plastic and the pathway taken at any instant in a cell depends on the activity state, mode of stimulation and the molecular machinery involved. Although it was first shown over four decades ago that the SVs were locally recycled, the precise pathway taken during vesicle recycling is still being debated and intensively studied (Heuser and Reese, 1973). The different endocytic pathways discussed so far include clathrin mediate endocytosis, bulk endocytosis, clathrin independent fast endocytosis, ultrafast endocytosis and direct retrieval of vesicle via fast closure of fusion pore (kiss-and-run) (Figure 3) (Soykan et al., 2016).

Clathrin-mediated endocytosis (CME) is one of the best characterized endocytic pathway which involves vesicle formation mediated by coat assembly (Saheki and De Camilli, 2012; Milosevic, 2018). However, CME process may be slow and cannot cope with internalization during certain physiological processes. Following a strong stimulus burst or certain specialized systems like ribbon synapse (Paillart et al., 2003), the excessive PM is removed from the surface by a different pathway called bulk endocytosis. This pathway involved internalization of endocytic vacuole in the first step followed by formation of SVs (Holt et al., 2003; Wu and Wu, 2007). Another pathway involves direct reformation of vesicles by a fast closure of fusion pore without full collapse of the vesicle membrane and is called kiss-and-run pathway (Gandhi and Stevens, 2003; Zhang et al., 2009). 


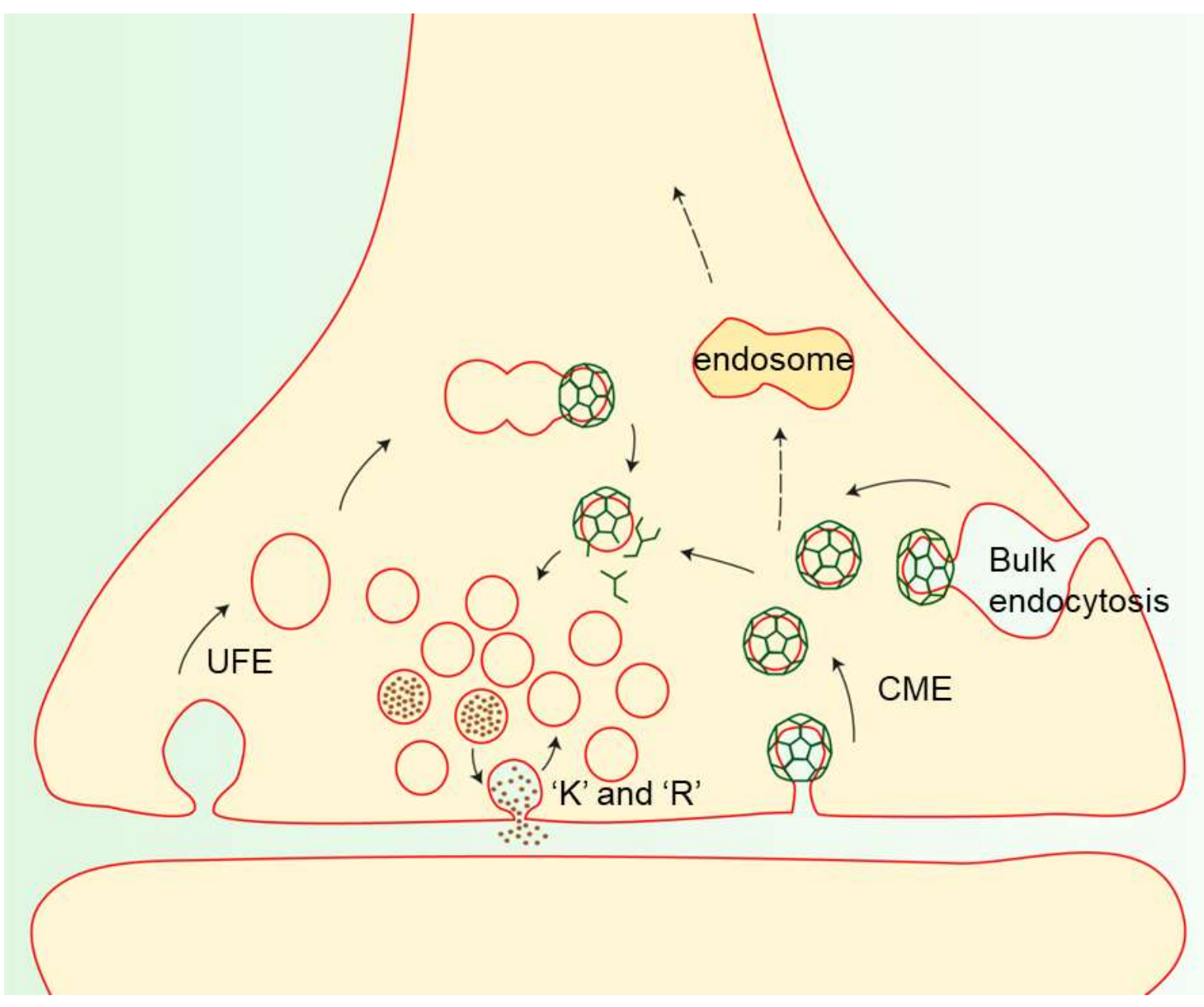

Figure 3 Pathways of endocytosis at the neuronal synapse

Endocytosis at the neuronal synapse following a fusion event can take different routes depending on the need of the cell, activity state and machineries involved in the process. Classically, SV proteins added by full fusion on the PM are recognized by the adaptors and retrieved by clathrin mediated endocytic pathway, which may take up to 30s. A strong stimulus results in bulk endocytosis wherein the excessive membrane is first internalized and then vesicles are formed from the endocytic intermediate (this may take 10s-1min and can result in non-homogenous SVs). Instead of full collapse, SVs can release NT and retrieved by a rapid closure of fusion pore (kiss-and-run pathway). Finally, ultrafast endocytosis, a newly suggested pathway mediates fast removal of the membrane $(\sim 100 \mathrm{~ms})$ from the surface followed by clathrin-mediated formation of SVs from endosomal intermediates (original illustration).

In situations which necessitate rapid cellular response, following a stress dependent hormone release or SV exocytosis, fast endocytic mechanisms like clathrin independent fast endocytosis 
(CIFE) and ultrafast endocytosis (UFE) mechanisms have been identified and their molecular machines are currently being revealed (Watanabe and Boucrot, 2017). The existence of a slow and fast endocytic mechanism has been proposed for a while now, supported by electrophysiological studies in neurons and chromaffin cells (von Gersdorff and Matthews, 1994; Artalejo et al., 1995). The fast recovery mechanism after a short stimulus occurs in the time scale of 1-2 seconds and suggested to be mediated by a clathrin-independent mechanism, whereas the slow form was observed after a strong stimulation, in the time scale of 15-20s and dependent on the clathrin machinery (von Gersdorff and Matthews, 1994; Smith et al., 2008). In neuroendocrine cells, where local recycling is not as essential as at the synapse, recycled vesicular proteins are mostly trafficked back to the Golgi, where new vesicles are formed again and trafficked to the PM for release. The contribution of CME at the presynaptic terminal under physiological conditions is currently being debated, although a role for clathrin-mediated reformation of vesicles from an endocytic intermediate, once the excessive membrane and proteins are engulfed is not excluded. I will briefly summarize the different endocytic pathways and effector molecules mediating the process in the following section.

\subsubsection{Clathrin-mediated endocytosis}

Clathrin mediated endocytosis signify the main pathway in many cell types with housekeeping function in membrane trafficking (Figure 3, 4). Most common mechanism of vesicle formation is budding induced by coat formation/protein complexes e.g clathrin at the synaptic terminal, COPI and COPII at the Golgi). CME is important for constitutive recycling of receptors in cells during trafficking. Various line of evidence argues for a role of $\mathrm{CME}$ in vesicle recycling process. Clathrincoated structures are less commonly spotted by EM at a resting terminal; however their numbers increase following stimulation that promotes vesicle recycling (Edeling et al., 2006; Owen et al., 2004). Clathrin, adaptor proteins like AP2 and other factors involved in the CME pathway are enriched at the terminal, where they are predominantly in cytosol and are recruited upon stimulation. Further, the composition of clathrin-coated vesicles isolated from the brain is similar to that of synaptic vesicles (Maycox et al., 1992). Perturbation of synaptic vesicle recycling experimentally also leads to a build of clathrin coated structures (Ferguson et al., 2007; Milosevic et al., 2011; Raimondi et al., 2011). At the neuronal synapses, the added proteins have to be 
sorted to make SV with precise protein composition. So, there must be a mechanism, to facilitate this protein sorting by endocytosis and clathrin mediated process is the most favorable candidate. It is yet to be understood at which point clathrin mediated vesicle formation comes into action (at PM or endosomal level at the synapse), but various evidences taken together suggests strongly for a role of this mechanism in regenerating SV locally at the synapse.

The formation of clathrin-coated vesicles is a highly dynamic process, which is orchestrated by spatially and temporally regulated protein-protein and protein-lipid interaction (reviewed in (Saheki and De Camilli, 2012), Figure 4). For simplicity, it can be considered a five steps process involving nucleation of the pit, cargo selection, coat assembly, vesicle scission followed by vesicle uncoating process. Nucleation of the pit involves interaction of the clathrin adaptor proteins and their accessory factors with $\mathrm{PI}(4,5) \mathrm{P}_{2}$ enriched on the PM and cargo proteins (cytosolic domains of SV proteins with sorting motifs) (Edeling et al., 2006; Owen et al., 2004). FCHo proteins (FBAR proteins) at the PM interacts with Eps 15 and intersectin, which subsequently recruits adaptor protein 2 (AP2) to the nucleation site (Henne et al., 2010). AP2 recruits several other adaptor proteins including AP180 (adaptor for VAMP2), stonin (along with AP2 helps to internalize synaptotagmin-1) and epsin, as well as accessory factors to form an adaptor protein hub which recruits cargo to their hub (cargo selection) (Diril et al., 2006; Ford et al., 2001, 2002). Adaptor protein hub further recruits clathrin light and heavy chain in the form of triskelia and their selfpolymerization leads to the formation of a distinctive lattice around the nucleation site (coat assembly) (Kirchhausen, 2000). Clathrin-coat, along with accessory proteins that can sense and induce curvature and cytoskeletal factors helps in the invagination of the coated pit and eventually leads to the formation of coated bud linked to the PM through a narrow neck. Coated vesicle is pinched off by a fission reaction mediated by GTPase protein dynamin (Ferguson and Camilli, 2012), recruited to the neck of the coated pit by BAR-domain containing proteins (Itoh and De Camilli, 2006). Dynamin undergoes self-polymerization and induce membrane scission upon GTP hydrolysis resulting in the formation of coated vesicle. Vesicle uncoating is rapidly initiated by the action of phosphatase synaptojanin-1, which hydrolyses $\mathrm{PI}(4,5) \mathrm{P}_{2}$ leading to the removal of adaptor proteins (Cremona et al., 1999; Schuske et al., 2003; Verstreken et al., 2003). Clathrin coat is disassembled by the action of auxilin which recruits ATPase Hsc70, leading to the formation of coat free vesicle, which contribute to formation of new SVs (Guan et al., 2010; Xing et al., 2010). 


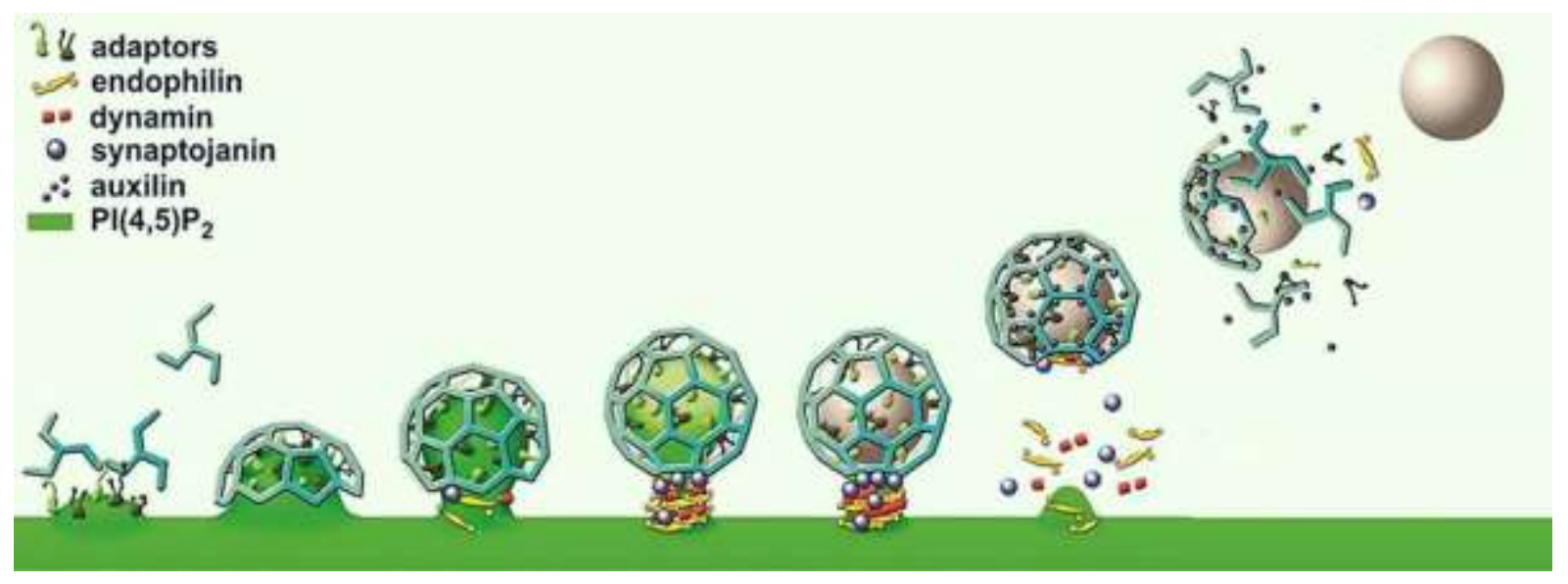

\section{Figure 4 Model of clathrin mediated endocytosis}

Clathrin mediated endocytic pathway depends on the adaptor proteins for cargo recognition and selection, followed by clathrin coat assembly resulting in an invaginated clathrin coated pit (CCP). CCPs are pinched by the GTPase enzyme dynamin, which is recruited to the neck by BAR domain containing protein like endophilinA, resulting in the formation of a coated vesicle (CCV). CCVs undergo rapid uncoating mediated by phosphatase synaptojanin-1, auxilin and Hsc70 resulting in the formation of a SV (adapted from Milosevic, 2018).

The fate of the uncoated vesicle was initially suggested to fuse with an endosome, from which new SV would be formed (Heuser and Reese, 1973). However, a direct recycling mechanism where SVs were derived by uncoating of CCV was also proposed (Takei, 1996). Newly formed vesicles/precursors of SVs (membrane and proteins) were suggested to merge with the recycling vesicles to form a single pool of proteins and membrane from which new SVs were regenerated at the synapse (Murthy and Camilli, 2003). Exocytosis of a set of vesicles does not necessarily lead to endocytosis of the same. The precise mechanism of how SVs are formed from uncoated vesicles is still not yet clear. 


\subsubsection{Bulk endocytosis}

This form of endocytosis occurs most commonly when a strong stimulation results in the fusion of number of vesicles within a short time span (Hayashi et al., 2008). The clathrin machinery would fail to buffer enough protein for efficient recycling after such an event. So, the excess membrane added is recaptured from the PM by membrane infoldings by a rather non-selective mechanism (Figure 3). However, their fission from PM and formation of intracellular cisternae (endocytic intermediates) implies it occurs by an active mechanism. Endosome-like-intermediates disappear eventually while new SVs are formed (Clayton et al., 2008).

The molecular mechanism of bulk endocytosis is not yet completely understood. Fission of the large cisternae from the PM seems to be independent of Dynamin, since such mechanisms occur at Dynamin1 KO terminals, where CME is severely compromised (Ferguson et al., 2007). The potential candidates that could mediate such a fission process include the actin cytoskeleton and BAR superfamily of proteins involved in membrane deformation (Itoh and De Camilli, 2006). Several scenarios for the conversion of cisternae like endocytic intermediates into SVs have been proposed, but the exact mechanism of action is not yet known (Saheki and De Camilli, 2012). SVs could be formed from the intermediates by a coat-mediated mechanism. However, it is known now that clathrin coat mediated vesicle formation requires $\mathrm{PI}(4,5) \mathrm{P}_{2}$, but, whether this machinery can be employed at the endocytic intermediate compartment remains to be explored. Other alternative mechanisms proposed includes formation of SVs by a novel protein- or lipid-mediated process independent of the clathrin coat, or by CME after the endosome fuses with the PM again. Formation of vesicles independent of classical coats raises the question about how the cargo can be sorted to form new SVs with correct protein composition.

\subsubsection{Clathrin-independent fast endocytosis}

CME is insufficient to support the demand during high intensity stimulation and the coat-mediated process is typically slow for certain kind of stimuli. This is not because of the low concentration of available clathrin in the cytosol, but is limited due to the rate of cargo selection, adaptor and triskelia activation rate and recruitment rate from the cytosol. Several forms of clathrin 
independent fast endocytosis have been reported till date, but an understanding of their mechanism of action lags behind mainly because of the following reasons: (1) Clathrin independent endocytic pathway is activated upon specific stimuli and is not constitutively active in the cell. (2). Proteins involved may not be specific to this pathway (3). CIE may be too fast to be recorded by classical methods used to study endocytosis so far (Watanabe and Boucrot, 2017).

Among the several described fast endocytic pathways, fast endophilin-mediated endocytosis (FEME) is a novel clathrin-independent mechanism, which involves the endocytic protein endophilin in the formation of vesicles quickly in the time scale of 1-10s (Boucrot et al., 2015). It is not constitutively active and initiated upon activation of specific receptors and toxins, but the mechanism of internalization is likely not identical. FEME is supported by specific protein machinery and membrane domain in the cells. The process is characterized by the speed with which endocytic intermediate following receptor activation are formed, transient nature of their activity and most importantly, coat-less tubule-vesicular morphology. Endophilin is enriched on certain membrane domains in the cell, which can support a rapid response upon receptor activation and in the absence of stimulus, and subsequently the foci disassemble within a few seconds (Renard et al., 2015). Endophilin can bind to an activated receptor as well as accessory proteins like dynamin and synaptojanin through its SH3 domain. BAR domain mediates curvature sensing as well as membrane deformation, apart from assisting dynamin in the scission process. Endophilin's role is supported by the upstream action of actin polymerization, mediated by Rac1 and Cdc42, leading to enrichment of endophilin and priming the cell for FEME (Boucrot et al., 2015). A comprehensive understanding of this pathway that supports specific stimuli and targeted receptor uptake and the detailed mechanism are yet to be identified.

\subsubsection{Ultra-fast endocytosis}

CME was suggested as the predominant pathway at the synaptic terminal; however, the recent works using an innovative electron microscopic technique suggests an alternative rapid pathway for recycling called ultra-fast endocytosis (Watanabe et al., 2013a, 2013b). The experimental technique involves optogenetic stimulation of the neuron to induce transmission followed by rapid 
freezing at defined time points to capture the membrane dynamics induced by the stimulus. Findings from this line of research suggest that the membrane is removed within $100 \mathrm{~ms}$ following a single stimulus (in the physiological range). The amount of membrane internalized is similar to that added during exocytosis, suggesting that this pathway is likely compensatory. This pathway is independent of action of clathrin at the PM (Watanabe et al., 2013a, 2013b, 2014) (Watanabe 2013 a, b, 2014). The rapidly internalized membrane fuses with the endosome, where clathrin acts to form SVs from endosomal intermediates (Watanabe et al., 2014) (Figure 3).

The molecular mechanisms of UFE are not well understood. Some of the key factors and molecular determinants of the pathway have been discovered so far. Calcium influx alone was not sufficient to induce UFE at the synapse and addition of membrane is a pre-requisite to initiate UFE, as observed in exocytosis-deficient munc13 mutants. F-actin is also important for the process, however, it is not clear if it is important to maintain the surface tension or an active polymerization is essential at the site of endocytosis Vesicle is internalized after a fission process that is dynamin dependent in UFE (Watanabe et al., 2013a, 2013b). UFE is initiated at specific sites lateral to fusion sites, probably at specialized membrane sites with specific lipid or protein organizations (Watanabe et al., 2013b). Recent evidences also show that endophilin and phosphatase synaptojanin are involved in UFE pathway of vesicle recycling (Watanabe et al., 2018).

Over the years, many pathways of vesicle recycling have been identified. This depends on the model organism, protein machinery, advancement in experimental techniques etc. Although the term SV recycling was coined almost four decades ago, the precise pathway taken by the vesicle following a particular stimulus is still being debated to-date. Nevertheless, the consensus is towards viewing the recycling mechanism as a two-step process, where SV membrane retrieval precedes SV formation. The process of membrane retrieval from PM can be clathrin dependent (classical CME) as well as independent of clathrin (as in the case of FEME and UFE). In the successive step, SVs are formed from endocytic intermediates via clathrin and this process can happen at the PM or from endosomes (Soykan et al., 2016; Watanabe et al., 2018). 


\subsubsection{Vesicle acidification and neurotransmitter uptake}

The next step in the recycling process is filling of the newly formed vesicles from endocytosis with cargo molecule (NTs or hormones), which helps to maintain the signal transduction process (Südhof, 2012). Irrespective of the pathway taken by the vesicle for endocytosis and SV formation, SVs have to load thousands of NT molecules to sustain neurotransmission. SVs are filled with excitatory NT like glutamate or inhibitory NTs like y-amino butyric acid (GABA) or glycine (Edwards, 2007; Takamori et al., 2000). NT present in the cytosol at the terminal is accumulated in the SVs for the release process. The refilling step of the vesicle formed by endocytosis includes two components namely vacuolar $\mathrm{H}^{+}$-ATPase (v-ATPase) and vesicular neurotransmitter transporters. The electrochemical gradient $\left(\Delta \mu \mathrm{H}^{+}\right)$is created by the action of $v$-ATPase, which is the proton pump on the vesicle (Hnasko and Edwards, 2012). V-ATPase essentially pumps the proton $\left(\mathrm{H}^{+}\right)$into the vesicle lumen, in an ATP dependent mechanism. Transporters of different NTs use this gradient created by the v-ATPase to pump the NTs into the vesicle. Although described as two-step process, it is likely occurring simultaneously under physiological conditions. The process of NT refilling is probably even overlapping with the endocytic process; however, it remains to be understood how the processes are linked and regulated. I will describe about these two steps in the next section, briefly discussing about the NT uptake mechanism and focus mainly on the v-ATPase and regulation of vesicle acidification in the recycling process due to relevance for chapter 3.2 of the thesis.

\subsubsection{Vesicle acidification by v-ATPase and its regulation}

\subsection{Electrochemical gradient}

Proton influx from the cytoplasm into the SV lumen generates a $\mathrm{pH}$ gradient across the membrane $(\Delta \mathrm{pH})$ that accounts for the more acidic environment of the SVs. It also results in the formation of membrane potential $(\Delta \Psi)$, which results in a positive voltage inside the vesicle. The two components combined together are referred to as the electrochemical gradient $\left(\Delta \mu \mathrm{H}^{+}\right)$or the proton motive force (pmf). Vacuolar $\mathrm{H}^{+}$-ATPase ( $\mathrm{v}$-ATPase) is an electrogenic pump and actively pumps the proton inside the vesicle by overcoming the opposing energy from the two components of $\Delta \mu \mathrm{H}^{+}$(Farsi et al., 2017). The electrochemical gradient across SV changes during the SV 
recycling process and $\Delta \mu \mathrm{H}^{+}$is made and dissipated during the exo-endocytic cycles. Net gradient is determined as a balance between $\Delta \Psi$ and $\Delta \mathrm{pH}$ in the vesicle and is influenced by other ion transports as well. $\mathrm{Cl}^{-}$ions affect the balance between the two components. In the absence of any $\mathrm{Cl}^{-}, \Delta \Psi$ is dominant as accumulation of $\mathrm{H}^{+}$leads to build up of a positive voltage in the vesicles. However, influx of $\mathrm{Cl}^{-}$counteracts the $\mathrm{H}^{+}$, thereby supporting the influx of more $\mathrm{H}^{+}$and thereby a net increase in $\Delta \mathrm{pH}$ (Blakely and Edwards, 2012). Both $\Delta \Psi$ and $\Delta \mathrm{pH}$ can be monitored by using fluorescent dyes. Also, the components of the $\Delta \mu \mathrm{H}^{+}$can be manipulated with ionophores like valinomycin, FCCP, CCCP, nigercin and ammonia (NH3) (Egashira et al., 2015).

The lumen of SVs is acidified to a pH of $\sim 5.7$ by the v-ATPase (Miesenböck et al., 1998). Considering the size of SVs, only a few protons are enough to establish the $\Delta \mu \mathrm{H}+$ across the vesicular membrane. However, other factors affect the net proton movement across the membrane and membrane potential. Regulatory factors that influence $\Delta \mu \mathrm{H}+$ include proton efflux from the vesicle lumen, gradients of other ions across the membrane among others (reviewed in Farsi et al., 2017). Ion gradient, apart from that of proton across the membrane affect the $\Delta \mu \mathrm{H}+$. This gradient is created by extracellular ions trapped inside the vesicles after endocytosis, ion exchange by transporters as well as ion-proton exchangers found on vesicles. Commonly found exchangers include $\mathrm{Na}^{+} / \mathrm{H}^{+}$exchanger (NHE) and $\mathrm{Cl}^{-} / \mathrm{H}^{+}$exchanger (CICs) (Grønborg et al., 2010). Immobile charged species could be contributed by the amino acid side chain groups of SV protein from their luminal domain or the cargo protein itself (eg chromogranin in chromaffin granules) and affect the charge distribution across the membrane (Johnson, 1988). Vesicular lumen probably has a high buffering capacity since acidification process in reality involves pumping of more than 1000 protons, whereas only a single proton molecule can establish the required gradient, considering the small size of vesicles. It was shown that the buffering capacity of vesicles increases with decreasing $\mathrm{pH}$ (Farsi et al., 2016). During the acidification process, as the $\mathrm{pH}$ of the vesicle approaches 5.7, the ratio of proton influx to ATP hydrolyzed reduces. Vesicle ceases to acidify further, although proton is still pumped into the vesicle, suggesting that the balance is achieved between the influx and efflux pathway. Acidification of an organelle stops when the proton influx is in equilibrium with the efflux (Farsi et al., 2017). Thus the steady state $\mathrm{pH}$ of an organelle is determined by the proton permeability and offers an explanation for different organelles achieving different steady state $\mathrm{pH}$, despite having the same v-ATPase pump (Van Dyke and Belcher, 1994). 


\subsection{Structure of v-ATPase}

Vacuolar $\mathrm{H}^{+}$-ATPase (v-ATPase) are large multi-subunit complexes that act as ATP-driven proton pumps. They are present on the membranes of all organelles that acidify as well as transport protons across the PM (Forgac, 2007). V-ATPase complex can be divided into two functional units, namely cytosolic $V_{1}$ domain and the membrane bound $V_{0}$ domain. The multi-protein complex contains 14 sub-units, some of which are regulatory sub-units with differential expression in different cell type and tissues (Forgac, 2007). Also, the isoform specificity helps to target the complex to the correct membrane. $V_{1}$ unit of v-ATPase support ATP hydrolysis while the integral $V_{0}$ domain helps to translocate proton across the membrane (Toei et al., 2010). The structure of v-ATPase is evolutionarily conserved and resembles mitochondrial $F_{0} / F_{1}$ ATPase. The $V_{1}$ sector consists of 8 sub-units $(A-H)$, among which 3 copies each of sub-unit $A$ and $B$ form the catalytic hexamer and their interface provide the site for ATP hydrolysis. $V_{0}$ domain has 6 sub-units (a, c, $c^{\prime}, c^{\prime \prime}, \mathrm{d}$ and e) and forms a ring with the proteolipid subunits through which $\mathrm{H}^{+}$are moved (Marshansky et al., 2014) (see Figure 5A). The peripheral stalk (formed by the accessory subunits $\mathrm{C}, \mathrm{H}, \mathrm{E}$ and $\mathrm{G}$ ) helps to hold the $\mathrm{V}_{1}$ complex of the holoenzyme in place (Muench et al., 2009). A central rotational stalk (made up of the ring along with sub-units $d$ of $V_{0}$ and $D, F$ of $V_{1}$ ) connects the two functional units and forms the rotatory unit of the complex (Arata et al., 2002; Iwata et al., 2004). Hydrolysis of three ATP molecules generates the free energy to transport six protons into the vesicle lumen. The free energy of hydrolysis drives rotation of the catalytic unit, which is coupled to the membrane bound channel-forming subunits, thereby leading to $\mathrm{H}^{+}$ translocation. The subunits undergo transition between three rotational states and their conformational changes orchestrate the $\mathrm{H}^{+}$translocation at the expense of ATP (Zhao et al., 2015). The number of $\mathrm{H}^{+}$effectively transported into the lumen upon ATP hydrolysis was variable and also dependent on $\Delta \mathrm{pH}$ (Kettner et al., 2003). 
A

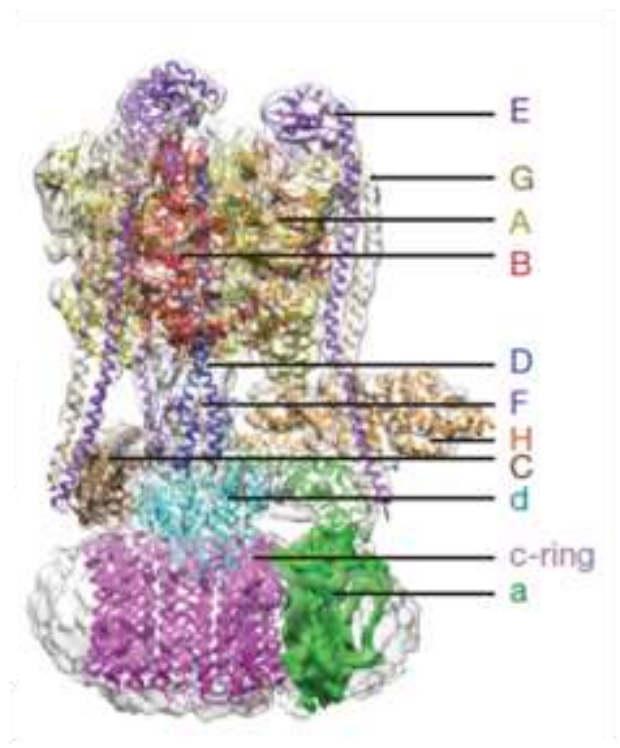

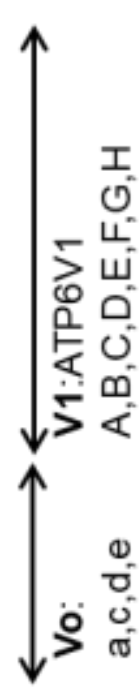

B

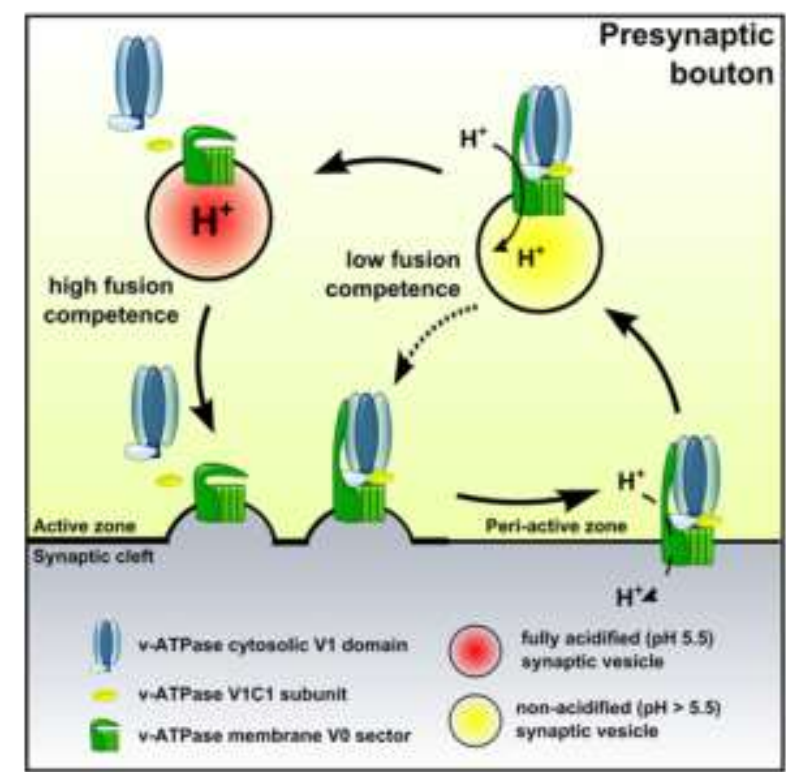

C

\section{vATPase is inactive in the presence of clathrin coat \\ vATPase is active upon vesicle uncoating}

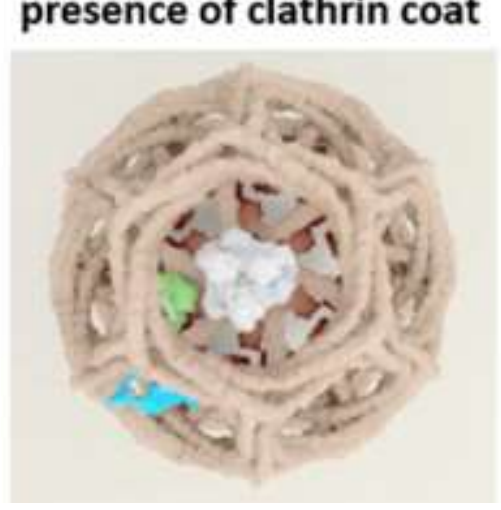

\section{assembly of clathrin coat inactivates vATPase}

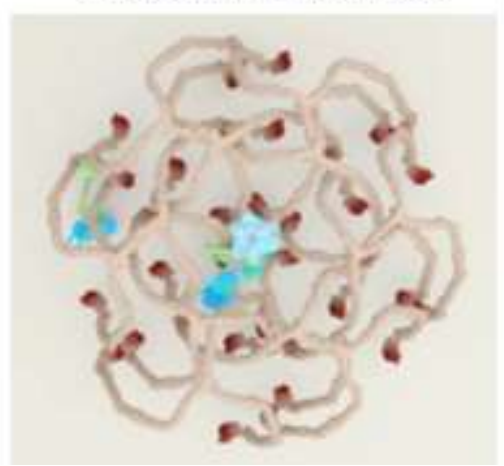

Figure 5 Structure of v-ATPase and its regulation during SV recycling

Structure of v-ATPase showing the various sub-units that form the complex on the vesicles (docked atomic models of the subunits). Cytosolic V1 complex includes ATP6V1 A, B, C, D, E, F, G, H subunits, whereas the membrane bound Vo comprises of a, c, $d$ and e subunits (Zhao et al., 2015). (B) A proposed model for $v$ ATPase disassembly/assembly coupled to exo- and endocytosis. V-ATPase pumps proton into the SV lumen to generate the electrochemical gradient for NT (re-)filling. When a vesicle is fully filled and become fusion competent, V1 sector as well as V1C1 diassembles from Vo sector and these vesicle preferentially undergo fusion at the PM. Non-acidified SVs with the completely assembled V-ATPase complex were suggested to have a low fusion competence (adapted from Bodzęta et al., 2017). (C) Model of v-ATPase 
activity regulation during SV recycling by the clathrin coat. The solved structures of v-ATPase, clathrin triskelia and AP2 complex were used to find out v-ATPase fits within the holes in the clathrin lattice. (Plasma membrane is indicated in light beige, clathrin triskelia in dark beige/brown; v-ATPase complex in gray (when inactive), light blue (when active) and dark green (V1H-subunit); AP2 complex in purple/blue/light green). Recognition of cargo by AP2 changes its conformation and recruits clathrin, which starts to build the clathrin coat around the v-ATPase complex. Insertion of the last triskelion of clathrin ring would collide with $\mathrm{V} 1 \mathrm{H}$, the regulatory subunit of v-ATPase, resulting in a hypothesis that the displacement of regulatory $\mathrm{V} 1 \mathrm{H}$-subunit inwards results in the block of the v-ATPase activity (adapted from Farsi et al., 2018).

\subsection{Regulation of v-ATPase}

v-ATPase is a multi-subunit protein complex that is crucial to the basic cellular function (Jefferies et al., 2008). It is reasonable that such a complex is expected to respond to various regulatory factors. However, the molecular mechanisms of regulation of the proton pump are far from understood (Beyenbach and Wieczorek, 2006). Initial ideas on physiological regulation of vATPase came from studies in yeast, where glucose starvation caused the dissociation of $V_{1}$ subunit from Vo sector. Glucose deprivation for a short time like 5 minutes led to dissociation of $70 \%$ of the complex and this process was reversible (Kane, 1995). Holoenzyme associationdissociation cycle, although deemed a universal v-ATPase regulatory mechanism, is not

understood fully and there are contradictory evidences for this to be true for SVs (Beyenbach and Wieczorek, 2006).

Most of the current knowledge on regulation of v-ATPase stems from detailed studies in yeast. Glucose induced assembly was supported by a heterotrimeric protein complex named RAVE (Regulator of the $\mathrm{H}^{+}$-ATPase of Vacuolar and Endosomal membrane) complex. RAVE complex binds V1 functional unit in the cytoplasm in yeast (Smardon et al., 2002). Another mechanism of regulation could be through differential expression of the genes encoding for v-ATPase sub-units. Most of the essential sub-units in yeast are encoded by a single gene and knock-out of the single copy of gene was lethal (Nelson, 2003). When a sub-unit is encoded by more than one gene or by alternative splicing, the isoforms are targeted usually to different tissues or organelle within the cell. In vertebrates, four isoforms of subunit a have been reported. The different isoforms have cell-type and tissue specificity (Toei et al., 2010). A genetic perturbation of the subunits gives rise to inherited disorders mostly, if it was not lethal. In renal epithelial cells, glucose induced 
activation of phosphatidylinositol 3-kinase (PI3K) signaling pathway leads to assembly of $v$ ATPase (Sautin et al., 2005).

In the v-ATPase assembly/disassembly cycle, $V_{1}$ sub-complex dissociates from $V_{0}$ into the cytoplasm. However, sub-unit $C$ seems to be special since it is released from $V_{1}$ upon $V_{0}-V_{1}$ disassembly. This suggests a regulatory role for $C$ sub-unit in holoenzyme disassembly/ reassembly (Beyenbach and Wieczorek, 2006). It is suggested to bind $V_{0}$ ad $V_{1}$ domains, by interacting with $E$ and $G$ subunit of $V_{1}$ and a subunit of $V_{0}$ (Inoue and Forgac, 2005). In yeast, structural changes in $\mathrm{C}$ sub-unit depended on ATP/ADP ratio, suggesting that it can act as glucose sensor and affect the stability of $V_{1} V_{0}$ holoenzyme (Armbrüster et al., 2005). There are also studies about interaction between C sub-unit and actin filaments (Vitavska et al., 2005), suggesting a novel function for F-actin in helping the v-ATPase stator to withstand the torque created by the rotating units.

Understanding the molecular mechanisms of v-ATPase regulation is fundamental to cellular processes. Regulators of the proton pump apart from the above discussed possibilities like kinases, phophotase are largely unknown. How did the v-ATPase emerge to have diverse functions ranging from exocytosis, endocytosis, degradation of macromolecule, membrane trafficking, protein sorting, recycling of NT, energizing membrane and transport systems? In the various organelles that v-ATPase are involved, how do they collaborate with other proteins (channels, pumps, carriers) in the transport process are some of the open questions central to cellular membrane trafficking pathways.

In the SV recycling process, v-ATPase has a central role in generating the electrochemical gradient, yet their complete function at the synapse is not well understood. Besides its well established role in proton translocation, v-ATPase has been implicated in non-canonical functions (reviewed in Maxson and Grinstein, 2014) like neurotransmitter release (Heisinger et al., 2005), membrane fusion process (Peters et al., 2001; Strasser et al., 2011) and exocytosis (Liégeois et al., 2006). $V_{0}$ domain was proposed to form a proteolipid channel at the fusion site during membrane fusion events in yeast (Peters et al., 2001). In Drosophila, mutations in vha100-1 (subunit a of $\mathrm{V}_{\mathrm{o}}$ ) lead to an accumulation of vesicles due to a release deficit without NT loading problems and further supported a role for subunit $\mathrm{V}_{\mathrm{o}}$ a downstream of the SNAREs in the fusion process (Hiesinger et al., 2005). Several studies along this line pointed to a role for v-TAPase in 
fusion process, independent of proton transport function. However, long-term effect of mutations or perturbations of v-ATPase subunit could affect membrane or protein trafficking. To this end, acute photo-inactivation of $V_{0}$ a1-I subunit resulted in a rapid decrease in NT release in neurons and catecholamine release in chromaffin cells (Poëa-Guyon et al., 2013). Further, it was suggested that $\mathrm{V}_{\mathrm{o}}$ acts as a pH sensor of the endo-lysosomal system (Hurtado-Lorenzo et al., 2006) as well as intragranular $\mathrm{pH}$ sensor that regulates exocytosis machinery (Poëa-Guyon et al., 2013). This second function of v-ATPase in release process raised a possibility that membrane domain could potentially help the exocytic machinery to distinguish between fully acidified and loaded vesicles from vesicles undergoing NT loading. Molecular link between v-ATPase and SNARE mediated fusion was strengthened by reported direct interaction between synaptobrevin2 and $\mathrm{V}_{0} \mathrm{C}$ subunit (Giovanni et al., 2010). Optogenetic control of vesicle acidification provided the means to understand the interplay between vesicular fill state and exocytosis. Although partially filled vesicles could be released, incompletely filled vesicles had a lower release probability, suggesting that vesicles with more content are preferentially exocytosed at the synapse (Rost et al., 2015). Such tools will help to understand the role of intraluminal $\mathrm{pH}$ in organelle function. In an attempt to understand the molecular mechanisms linking v-ATPase activity regulation and SV exocytosis in hippocampal neurons, $\mathrm{V}_{0} \mathrm{C}$ subunit tagged with $\mathrm{pH}$ sensitive $\mathrm{pHluorin}$ was used as a reporter to follow presynaptic activity (Bodzęta et al., 2017). v-ATPase assembly was found to be tightly regulated by $\mathrm{pH}$ of the vesicle and coupled to exo-endocytosis cycle. $\mathrm{V}_{1}$ domain was not found on fully acidified vesicles that were fusing and suggested that holoenzyme assembly provides an alternative mechanism for favorable fusion of fully acidified and filled vesicles (Bodzęta et al., 2017).

Although $\mathrm{V}_{0} / \mathrm{V}_{1}$ assembly offers a regulatory mechanism for $\mathrm{v}$-ATPase activity in cellular trafficking organelles, it is not clear if this holds true for SVs during vesicle recycling process. Firstly, synaptic vesicles isolated from mammalian brain were found to contain only 1-2 copies of v-ATPase. Further, all the subunits of the v-ATPase (subunits of both $V_{0}$ and $V_{1}$ complex were found on SVS by mass spectrometry) (Takamori et al., 2006). Although one proton pump per vesicle can cater to the energetics of NT refilling, it suggests for a highly regulated trafficking process to sort one v-ATPase complex per vesicle. Further, single vesicle imaging of SVs expressing $\mathrm{pH}$ sensitive synaptopHluorin showed that isolated vesicles can undergo acidification upon adding ATP alone, without requiring any other component (Farsi et al., 2016). These two reports strongly suggest 
that v-ATPase complex contain the $\mathrm{V}_{\mathrm{o}}$ as well as $\mathrm{V}_{1}$ domain on isolated $\mathrm{SV}$ s. Although majority of SVs contain a functional V-ATPase, it cannot be ruled out that vesicles may lose V1 domain during the fusion process itself. This raises the question whether v-ATPase is fully active through the entire SV recycling process or regulated in the cycle of events. After the fusion step, vesicles undergo endocytosis, which involves formation of coated vesicles. While some reports suggested that CCVs do not acidify (Anderson and Orci, 1988; Anderson et al., 1984), others showed that acidification occurs in these vesicles (Forgac et al., 1983; Van Dyke and Belcher, 1994). To address this question, we isolated clathrin coated vesicles from mice and measured membrane potential as well the $\mathrm{pH}$ of these vesicles. Single vesicle measurements showed that CCV did not undergo acidification to the same extent as SVs (Farsi et al., 2018). Biochemical analysis showed that $\mathrm{V}_{\mathrm{o}}$ and $\mathrm{V}_{1}$ complex subunits were present on CCVs. Interestingly, the acidification in CCVs could be restored to SV levels by removing the clathrin coat alone.

Using the information about structure of clathrin coat around the vesicles as well as that of $v$ ATPase, we proposed a model in which clathrin coat formation around the vesicle inhibits $v$ ATPase activity by displacing the $\mathrm{V}_{1} \mathrm{H}$ subunit to accommodate $\mathrm{V}$-ATPase complex into the hexagons of CCV lattices (Farsi et al., 2018). We are yet to understand the molecular mechanism of $v$-ATPase activity regulation and additional players (if any) involved in the regulation process as well as when they are regulated in SV cycle. Interaction partner mapped using kidney tissues revealed v-ATPase interacting partners to be involved in the holoenzyme assembly, protein quality control, intracellular trafficking and v-ATPase specific accessory proteins (Merkulova et al., 2015). Among the v-ATPase specific accessory proteins were DMX-like 1(DMXL1), DMX- like 2 (DMXL2), WD- Repeat domain 7 (WDR7), NCOA7 (nuclear receptor coactivator 7) and oxidation resistance 1 (OXR1). Interestingly, a proteomic study to identify the true protein complex performed with DMXL2 revealed v-ATPase subunits as well as WDR7 as interacting partners (Li et al., 2012). DMXL2 and WDR7 (Rabconnectin-3a and 3b) form a subunit structure and could be potential candidates for v-ATPase regulation and I will summarize the current state of knowledge on this v-ATPase specific accessory protein in the end of this chapter. 


\subsubsection{Neurotransmitter uptake}

Vesicular transporters use the electrochemical gradient $(\Delta \mu \mathrm{H}+)$ generated by the v-ATPase to accumulate NT inside the vesicles. Transporters for specific NT are expressed in particular vesicles and are determinants of the NT phenotype of SVs. They can be broadly classified into three families of proteins namely solute carrier proteins (SLC) SLC17, SLC 18 and SLC 32 (Blakely and Edwards, 2012). Glutamate transporters belong to SLC17 family and three isoforms (vesicular glutamate transporter VGLUT1, 2 and 3) are encoded by the SLC17 A6-8 genes. Transporters for monoamines and acetyl choline are encoded by SLC18 family and include vesicular monoamine transporter (VMAT1 for monoamines and VMAT2 for histamines) and vesicular acetyl choline transporter (VAChT). SLC32 encodes for vesicular GABA transporter (VGAT), also known as vesicular inhibitory amino acid transporter (VIAAT) (reviewed in Takamori, 2015). The transporter is shared by inhibitory NTs GABA and glycine, found in both GABAergic and glycinergic neurons and the NTs were reported to compete for vesicular uptake (Wojcik et al., 2004). The extent of influence of $\Delta \Psi$ and $\Delta \mathrm{pH}$ on the NT uptake depends on the charge of the molecule at cytoplasmic $\mathrm{pH}$. Accordingly, the transport of monoamines (dopamine, serotonin, adrenalin and nor adrenalin) and acetyl choline depends mainly on $\Delta \mathrm{pH}$, whereas, negatively charged NT like glutamate depends mainly on $\Delta \boldsymbol{\psi}$ for its transport. GABA and glycine transport depends on both $\Delta \Psi$ and $\Delta \mathrm{pH}$ components (Blakely and Edwards, 2012). Regulation of NT uptake and refilling of vesicles is done by regulating the transporter itself, regulation of v-ATPase and/or mechanisms affecting electrochemical gradient (reviewed in Farsi et al., 2017). A key step to get an overview of the entire SV recycling involves decoding the spatial and temporal regulation of $\mathrm{V}$ ATPase function as well as NT refilling.

Understanding how a newly formed vesicle is filled with neurotransmitter and made fusion-ready is important in the context of synaptic transmission. Regulation of transmission process can be pre- or post-synaptically mediated. Among the other parameters, the amount of NT released or the quantal content affects the strength of release. Recent studies have shown that fully filled and acidified vesicles are preferentially released with a higher release probability. This was speculated to be a result of increased intravesicular osmolarity, higher vesicle membrane tension and hence increased fusion probability (Rost et al., 2015). Alternative mechanism mediated by assembly/disassembly cycle of v-ATPase has been proposed to favor fusion of fully filled vesicles 
(Bodzęta et al., 2017). This could be relevant for reliable synaptic transmission process, especially during high rates of vesicle turnover and sustained neuronal firing.

\subsubsection{SV clustering and maturation}

Vesicles at the synapse are organized into functionally distinct pools or clusters (Rizzoli and Betz, 2005). Vesicles that arrive from the de-novo synthesis pathway or locally formed by the recycling process are captured into these clusters. During prolonged stimulation, vesicles are mobilized from these clusters to ensure SV availability, when recycling process can become rate limiting. Synapsins are highly abundant, synapse enriched phosphoproteins that can reversibly associate with SVs and considered as the primary component responsible for SV clustering. Synapsin can bind SVs as well as actin cytoskeleton in phosphorylation dependent manner (Greengard et al., 1993). Synapsin interacts with peripheral SV protein Rab3 (Giovedì et al., 2004). Rab proteins are small $G$ proteins involved in modulation of membrane trafficking. Synapsins, along with other peripheral SV proteins like $\alpha$-synuclein (Vargas et al., 2017), Rab proteins and their interactors (Pavlos and Jahn, 2011) are likely controlling the SV clustering process. Additionally, some endocytic proteins (amphiphysin, dynamin, endophilin, intersectin-1 among others) have been found among the matrix components connecting SVs at resting state. Upon stimulation-induced dephosphorylation, they are translocated to the periactive zone for SV recycling (Shupliakov et al., 2011). Recently, matrix protein synapsin was shown to organize vesicles in clusters by liquidliquid phase separation, thereby suggesting vesicle clustering can be explained by phase separation principle at the synapse (Milovanovic et al., 2018). Several molecular players participating in the clustering of SVs have been identified; however, much remains to be understood about SV clusters in the context of vesicle recycling process at the synapse.

\subsection{Endo-Iysosomal pathway}

Endocytosis broadly involves internalization of cargos in a cell that includes receptor-ligand complexes, lipids, proteins, fluids, nutrients, bacteria, viruses among many others from PM. The cargo in the vesicular carries is formed by a budding reaction from one organelle and delivers 
their content to the target organelle by a fusion reaction in the endocytic as well as secretory pathway. The internalized vesicle then fuses with membrane delimited organelle called endosomes and goes through steps of sorting (Figure 6) (Helenius et al., 1983; reviewed in Huotari and Helenius, 2011; Wandinger-Ness and Zerial, 2014). Each organelle involved in the pathway is defined by specific set of Rabs and their effectors proteins, phosphoinositides and peripheral factors. Rab GTPases form a structural and functional regulatory domain, providing specificity to the SNARE complexes during membrane tethering and fusion (Zerial and McBride, 2001).

The cargo from the early endosome is primarily recycled back to PM via recycling endosomes, as in the case of some receptors (e.g. transferrin receptor). Alternatively, the endosome with the cargo can mature into a late endosomes, as it proceeds towards the peri-nuclear area. They are combined with lysosomal hydrolases and other components to form late endosome (LE) that acts as a feeder system to deliver the cargo as well as components to the lysosomes. The fusion with lysosomes results in the formation of endolysosomes where active degradation of proteins takes place, but this is a limited secondary pathway. Since the nerve terminal is separated from the soma, SV membrane and proteins undergo several rounds of recycling, without the need for new vesicle biogenesis constantly, communication with trans-Golgi network or targeting to the degradation pathway.

Endosome-like-structures have been identified at the synapse following a strong stimulation, since the first reports on SV recycling process (Heuser and Reese, 1973). However, it is not clear whether these endosome-like-structures are related to the classical endosomes of the endocytic tracking pathway and their role in SV recycling process remains unclear (Jähne et al., 2015). Endosomal SNARE proteins like syntaxin 6, VAMP4 and vti1a along with Rab5 have been found on synaptic endosomes, as well as on SVs, and suggested to mediate fusion of endocytosed vesicles with itself or with endosomes (de Hoop et al., 1994; Rizzoli et al., 2006; Takamori et al., 2006). Inhibition of SV fusion with the endosomes or interfering with the sorting machinery affected SV recycling process (Hoopmann et al., 2010). Although the complete mechanism is not understood, endosomes have been suggested to facilitate sorting of SVs during the recycling process to remove malfunctioning proteins or PM proteins and maintain fusion competence of vesicles ( reviewed in Jähne et al., 2015; Uytterhoeven et al., 2015). 


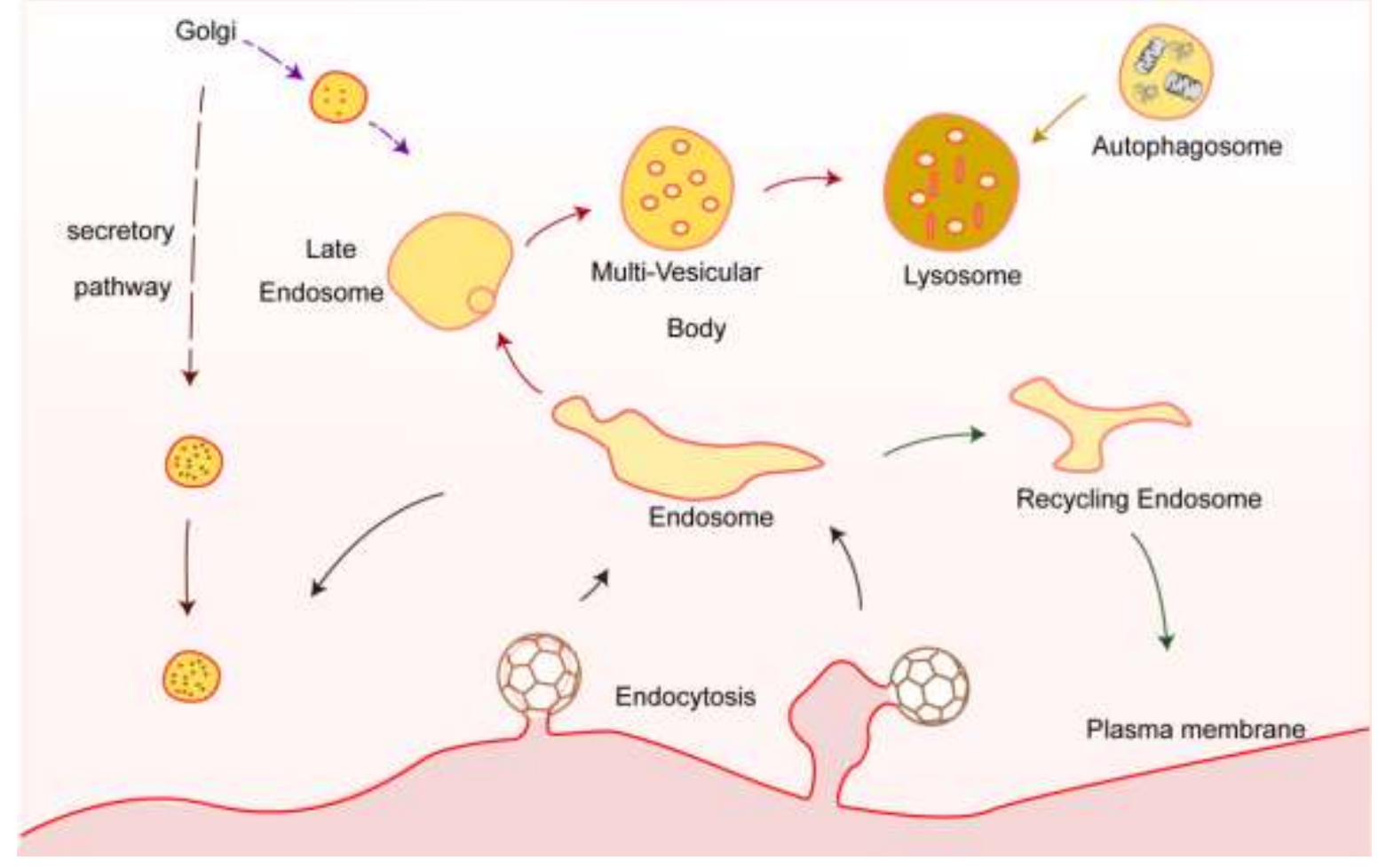

Figure 6 Cellular membrane trafficking pathway

Various routes taken by the retrieved protein and membrane in the membrane trafficking pathway. Endosomes act as the sorting station and proceed further along the recycling route or degradative route. Lysosomes act as a center where the endocytic, secretory and the autophagy pathway converge for macromolecule degradation, nutrient recycling and signaling (Original illustration).

The cargo targeted for degradation is destined to reach lysosomes. Lysosomes act as a center for macromolecule degradation and nutrient recycling in the trafficking pathway. Endocytic, secretory and autophagy pathway converge at lysosomes to mediate such functions in the cell (Figure 6) (Klumperman and Raposo, 2014). Subsequently, catabolic activity of the lysosomes yields the basic building blocks of the cell like amino acids, nucleic acids, sugars among others, especially in times of stress. Apart from this hydrolytic and recycling function, increasing evidence support a role for lysosomes as a signaling station to integrate cues from metabolic and signaling pathways in the cell (Perera and Zoncu, 2016). Optimal functioning of lysosomes therefore seems essential for neurons to meet the demands during metabolic activity and neurotransmission. 
Despite their central role in the trafficking pathway, the function of neuronal lysosomes is only beginning to be understood (see review Ferguson, 2018). New data supports the idea that lysosomes in distinct neuronal compartments support a specific function and are redistributed in the neurons depending on the ongoing changes in demand during activity. Recently, many endocytic proteins involved at PM like AP2 and endophilin have been attributed to have additional role in the autophagy pathway, thereby providing more links between the different routes and how they are regulated during aging (Azarnia Tehran et al., 2018). These findings report a new role for proteins involved in vesicle recycling process and an unanticipated link between machineries involved in synaptic transmission and neuronal proteostasis.

\subsection{Role of Endophilin-A in SV recycling}

Cellular membranes are important components of the membrane trafficking and SV recycling process. Membranes of the organelle are in a dynamic flux, as they change their shape and composition in the trafficking pathway and are influenced by the lipids in the membrane, cytoskeletal factors and cytosolic proteins, that can deform membranes (Itoh and De Camilli, 2006). Members of the superfamily of Bin-Amphiphysin-Rvs (BAR) domain-containing proteins play a vital role in membrane remodeling by inducing membrane curvature, stabilizing existing curvature and sensing the curvature of the membrane to recruit cytosolic proteins to the organelle (Daumke et al., 2014; Frost et al., 2009).

Endophilins belong to the BAR superfamily proteins and are one of the best studied endocytic adaptors orchestrating steps in CME as well as CIE processes (Saheki and De Camilli, 2012; Watanabe and Boucrot, 2017; Milosevic, 2018). In mammals, endophilin protein family include two sub-families, namely endophilin-A ( 1 1, A2 and A3) and endophilin-B (B1 and B2). Endophilin$B$ subfamily encoded by the genes SH3GLB1 and B2 respectively is implicated in autophagy process (Kjaerulff et al., 2011). Endophilin-A (henceforth endophilin) protein is encoded by three genes: SH3GL2 (endophilin 1, brain-enriched), SH3GL1 (endophilin 2, ubiquitously expressed) and SH3GL3 (endophilin 3, brain and testis-enriched) (Giachino et al., 1997; Ringstad et al., 1997; So et al., 2000). They contain an N-terminal BAR-domain that induce, stabilize as well as sense membrane curvature, and a C-terminal SH3-domain that mediates protein-protein interactions 
(Gallop et al., 2006; de Heuvel et al., 1997; Ringstad et al., 1997). Endocytic adaptor function is mediated by these two domains, wherein they are central player in a hub of protein network involved in membrane remodeling, cargo packing, bud constriction and recruitment of factors needed for fission and/or uncoating processes (Gallop et al., 2006; de Heuvel et al., 1997; Milosevic et al., 2011; Ringstad et al., 1997; Schuske et al., 2003; Takei, 1996; Verstreken et al., 2003). Endophilins' SH3 domain mediates protein-protein interaction that is crucial to various steps in SV recycling process. They are responsible for recruiting the GTPase protein dynamin to mediate membrane fission and phosphatase synaptojanin-1 to facilitate uncoating step (de Heuvel et al., 1997; Ringstad et al., 1997). In cells lacking dynamin, endophilin was found to be enriched in the neck region of coated pits and suggested that endophilins' recruitment to the clathrin-coated pit is upstream of dynamin (Ferguson et al., 2009; Perera et al., 2006). Genetic and targeted ablation studies in mammals as well as invertebrates showed that the lack of endophilin results in accumulation of CCVs and defective SV recycling at the synapse. This was reminiscent of the phenotype observed in the loss of $\mathrm{PI}(4,5) \mathrm{P}_{2}$ phosphatase synaptojanin-1 (Schuske et al., 2003; Verstreken et al., 2003; Milosevic et al., 2011). Besides the interaction with factors involved in $\mathrm{CME}$, endophilins were also found to interact with proteins of the cellular degradation machinery and implicated in neurodegenerative disorders like Parkin and Fbxo32/atrogin-1 (Cao et al., 2014; Murdoch et al., 2016; Soukup and Verstreken, 2017; Trempe et al., 2009).

Endophilin was also attributed to play a key role in CIE process, like fast endophilin-mediated endocytosis (FEME), which is not constitutive and is triggered following ligand mediated activation of specific receptors or toxins uptake (Boucrot et al., 2015; Renard et al., 2015). Most recently, endophilin was found to participate in ultrafast endocytosis, where the membrane added by a short stimulus is internalized within $100 \mathrm{~ms}$, without clathrin and vesicles are formed in a later step from endosomes mediated by clathrin (Watanabe et al., 2018). Taken together, endophilin is central to endocytosis and trafficking.

Role of endophilin as a central player in different pathways of endocytosis has been established in the last 20 years and detailed mechanism have been elucidated since the first report on the protein. However, indications for alternative function for endophilin at the synapse have been proposed. One such study reports vGLUT1 as interactor of endophilin (Vinatier et al., 2006). It 
was shown that vGLUT1 upon binding endophilin-A1 inhibits endophilin-mediated enhancement of release probability (Weston et al., 2011). This study suggested that endophilin-A1 acts a positive regulator of neurotransmitter release and argued for a role for the BAR membrane curvature sensing property to implement this in vGLUT1-positive synapses.

Endophilins, along with other endocytic factors (called dephosphins) were reported to be maintained in a matrix among SV clusters and recruited to the periactive zone upon stimulation (reviewed in Shupliakov, 2009). Intersectin is a multimodular scaffolding protein that interacts with proteins from the endocytic, cytosketal and exocytic machinery (dynamin, epsin, Cdc42 and even SNARE proteins SNAP-25 and SNAP-23 (Pechstein et al., 2010; Sakaba et al., 2013). It was proposed that it shuttles between active and periactive zone and orchestrates the coupling between exo-and endocytosis. Endophilin 1 was reported to interact with intersectin-1, mediated through the SH3-domains of both proteins (Pechstein et al., 2015), but the relevance of this interaction in the SV recycling process still remains to be understood. In C.elegans nerve terminal, endophilin was shown to be targeted to the site of endocytosis by exocytosis and undergo an association-dissociation cycle with the SVs (Bai et al., 2010). The authors suggested that this endophilin cycle might provide a mechanism for coupling exo- and endocytosis. Interestingly, lack of all three endophilin in mice resulted in impaired synaptic transmission and the synapses showed reduced SVs (Milosevic et al., 2011). It was not clear if these was due to defective recycling process as the process of endocytosis is tightly coupled to exocytosis in such synapses or from a direct role for endophilin in fusion process itself, or both. 
A

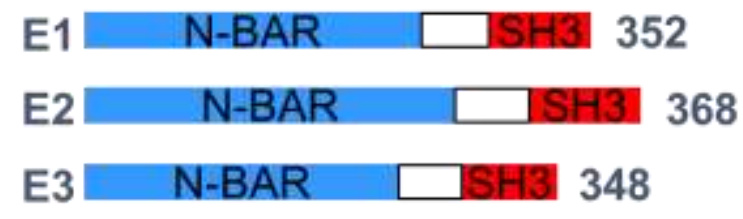

C
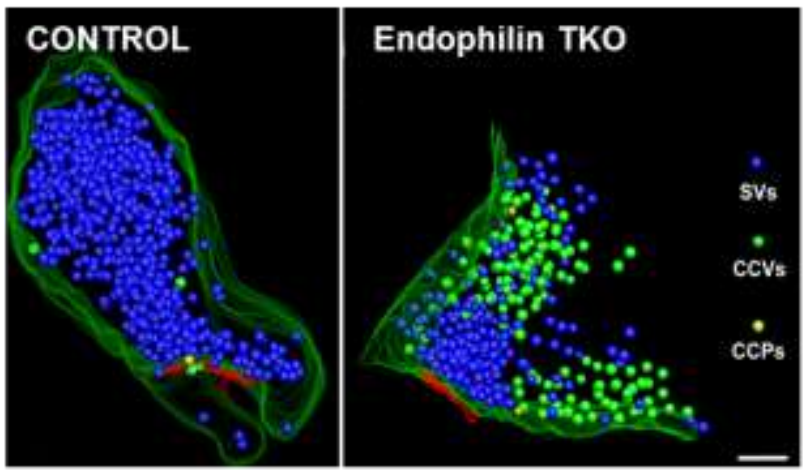

B

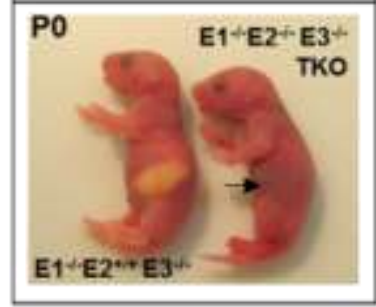

D

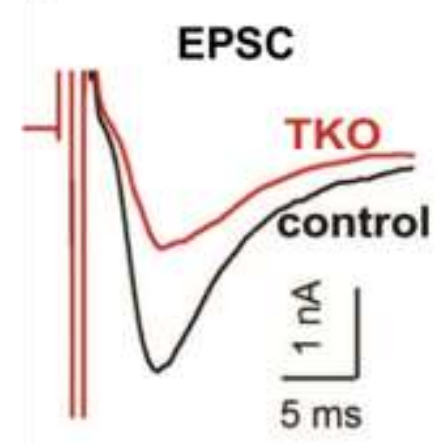

E

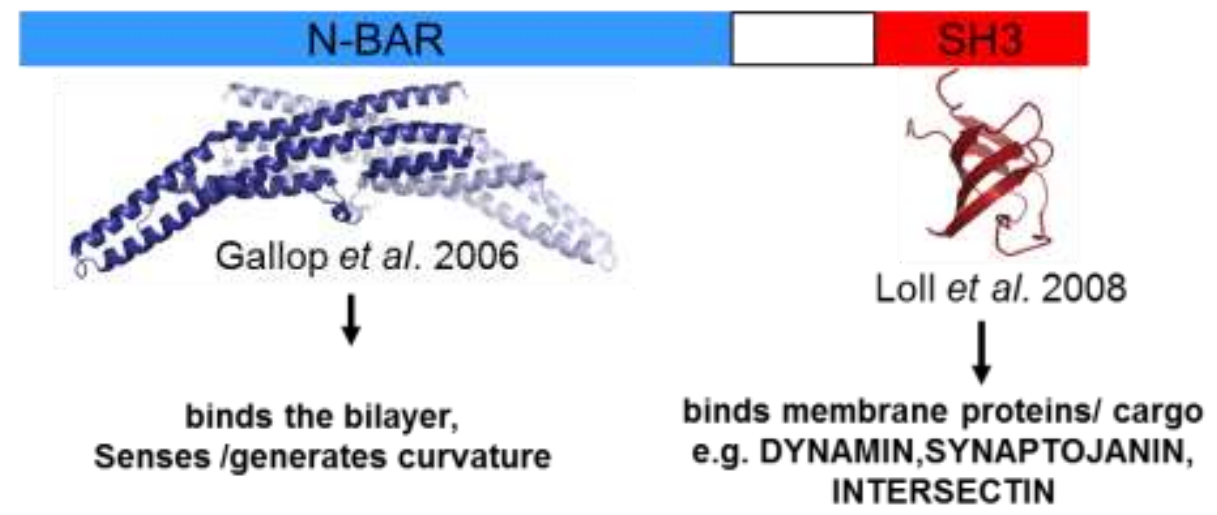

Figure 7 Structure and function of Endophilin in SV recycling

(A) Endophilin, belonging to the BAR super family includes $A 1, A 2$ and $A 3$ proteins. (B) Loss of all three endophilin (TKO) results in perinatal lethality in mice. (C) EM tomograms of Endophilin TKO synapses shows accumulation of clathrin coated vesicles due to defective SV recycling. (D) Synaptic transmission is impaired in endophilin TKO neurons. (E) Structure of endophilin showing its functional domain. It has a BAR domain that helps in membrane binding and curvature sensing, and a SH3 domain involved in proteinprotein interaction (adapted from (Gallop et al., 2006; Loll et al., 2008; Milosevic et al., 2011). 


\subsection{Rabconnectin-3a in vesicle recycling}

The precise mechanism of how and when a newly (re-)formed vesicle becomes a fusion competent SV is not yet known. Vesicles formed by recycling pathway are acidified, filled with NT and recruit peripheral SV marker proteins like Rab3 and synapsin that organize them in a cluster, thereby possibly deciding their fate to undergo stimulated exocytosis. Rab3 and synapsin are present on mature SVs, which are fusion competent and can be used as their molecular markers (Matteoli et al., 1991). Rab3, as discussed earlier belong to the Rab GTPase superfamily and facilitates calcium-triggered exocytosis (Pavlos and Jahn, 2011). Rab3 in the GTP bound state can bind to SVs peripherally and its recruitment to SVs depends on their GTP/GDP binding state (Fischer von Mollard et al., 1990). It is actively cycled at the synapse, regulated by Rab dissociation inhibitor (GDI), GDP/GTP exchange factor (GEF) and GTPase-activating protein (GAP). Rab3 on SVs further interact with Rim and Munc13 to facilitate triggered exocytosis (reviewed in Südhof and Rizo, 2011). During or after fusion, GTP is hydrolyzed, mediated by Rab3-GAP and Rab3-GDP dissociates from the SVs (Von Mollard et al., 1991). In the GDP bound state, Rab3 remains in the cytosol, in association with Rab dissociation inhibitor (GDI). Upon stimulation, Rab3 is activated by exchanging GDP for GTP by Rab3-GEF in association with an unidentified molecule. It is not known at which step Rab3-GEF and GAP are recruited in the SV recycling process.

A protein found to co-immunoprecipitate with Rab3-GEF and GAP was named Rabconnectin-3 (Nagano et al., 2002). Human Rabconnectin-3 (RC3) protein is $340 \mathrm{kDa}$, consists of 3,036 aa and encoded by DMX-like 2 (DMXL2) gene. Its primary structure was predicted to contain 12 WD repeat domains (Nagano et al., 2002). The protein was found to be enriched in brain and specifically in the SV crude fraction from rat brain extract. It was also found to localize at synapse from immunohistochemical analysis (Nagano et al., 2002) (Figure 8).

In a follow up study, another protein was identified to interact directly with Rab3-GEF and named Rabconnectin-3 $\beta$ (henceforth Rbcn-3b) (Kawabe et al., 2003). The previously identified subunit was renamed as Rabconnectin-3a (henceforth Rbcn-3a) and suggested to form a complex with Rbcn-3b. Both the sub-units interacted with Rab3-GAP indirectly. The partial sequence was determined and cDNA was cloned from the human cDNA library. Rbcn-3b consists of 1,490 AA, with a molecular weight of $163 \mathrm{kDa}$ and made up of seven WD domains. It was found to be 
abundantly expressed in the brain and formed a stable complex with Rbcn-3a; however, it is not clear if they form a stable complex on SV always or regulated during the SV cycle (Figure 8) (Kawabe et al., 2003).
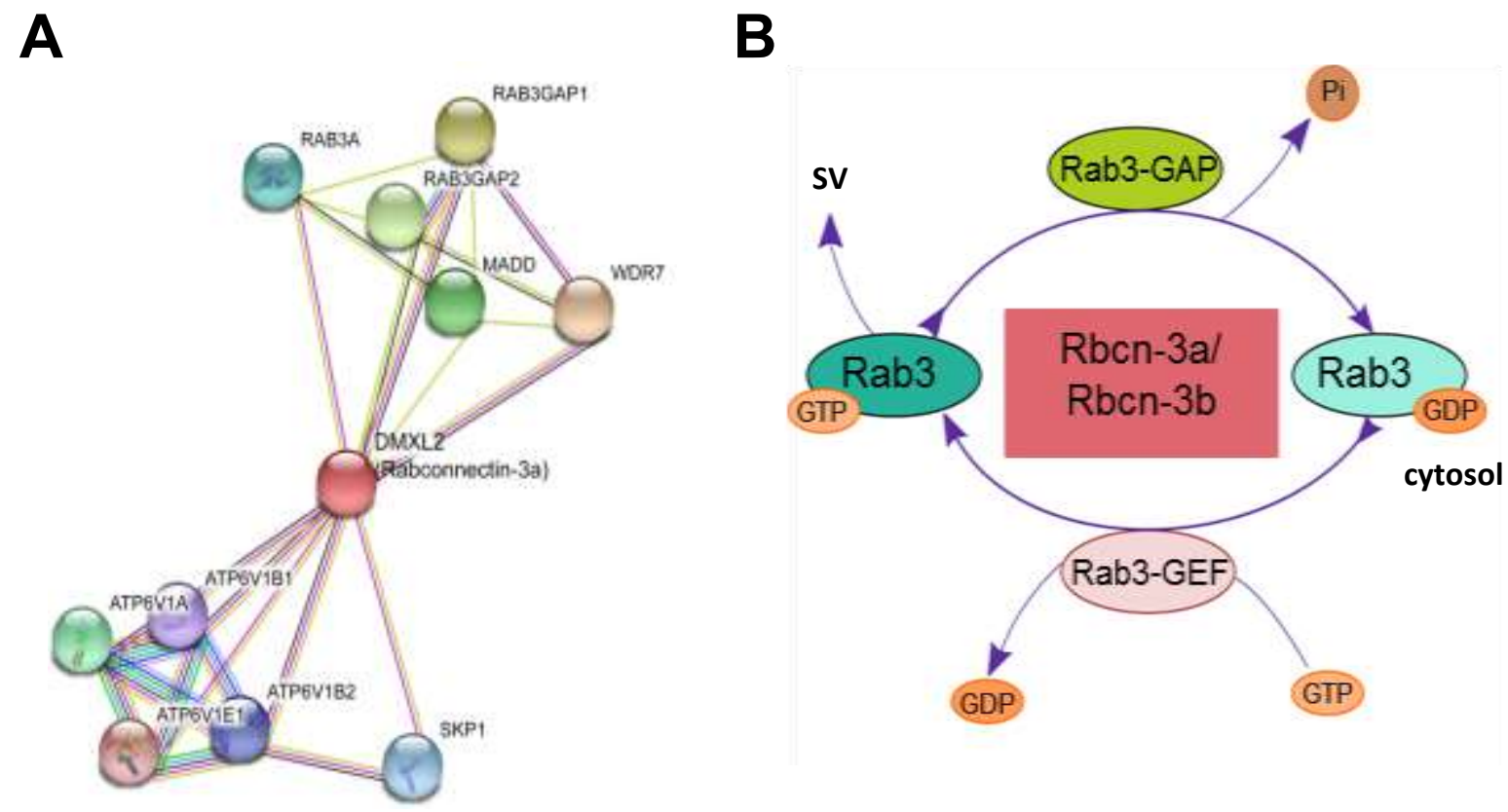

Figure 8 Molecular interactions of Rabconnectin-3a

(A) Rabconnectin-3a (encoded by the gene DMXL2) has been known to interact with Rab3 regulating factors as well as multiple sub-units of v-ATPase in various model organisms from yeast to mammals (interaction network in humans from STRING). (B) Rabconnectin-3 was found to co-immunoprecipitate with Rab3-GEF and GAP. Rab3 in the GTP bound state can bind to SVs peripherally. It is actively cycled at the synapse, regulated by Rab dissociation inhibitor (GDI), GDP/GTP exchange factor (GEF) and GTPaseactivating protein (GAP).

After these initial studies where they identified the proteins, there have been few studies to date on the role of Rbcn-3a using various model systems from yeast to mammals, and all relevant studies are quoted here (Figure 8). The yeast homolog of Rbcn-3a, Rav1, was found to regulate the assembly and activity of v-ATPase, as mentioned before in the section on regulation of $v$ ATPase (Seol et al., 2001; Smardon and Kane, 2007; Smardon et al., 2002). Rav1 is part of the 
RAVE complex, along with Rav2 and Skp1 is involved in glucose-induced assembly of v-ATPase complex in yeast (Seol et al., 2001). Loss of function of RAVE complex also affected the transport between early endosome and prevacuolar compartment in yeast (Sipos et al., 2004). Rav1p was identified as a central component of RAVE complex with distinct interaction sites for Rav2p, Skp1p, $V_{1} E$ or $G$ and $V_{1} C$ subunit of V-ATPase complex (Smardon and Kane, 2007; Smardon et al., 2002). Although homolog of Rav1 exists in eurkaryotes but Rav2 does not, another protein Rbcn-3b is found to form subunit structure with Rbcn-3a (Kawabe et al., 2003; Kraemer et al., 2000).

The fly's homolog of Rbcn-3a was reported to regulate Notch signalling and endocytic trafficking during development, primarily by acting through v-ATPase. In the Rbcn-3a mutants, Notch receptor and other membrane proteins were trapped in the late endosome and the phenotype was similar to that of V-ATPase mutant VhaAC39. Further, Drosophila Rbcn-3b coimmunoprecipitated with V1B and $\mathrm{H}$ subunit of V-ATPase complex (Yan et al., 2009). In a follow up study, Rbcn-3a was also found to regulate Notch signaling in mammalian cell line (Sethi et al., 2010). Thus, disrupting the v-ATPase or its regulator Rbcn-3 leads to an impaired Notch signaling in Drosophila.

Rbcn-3a was found to stabilize the activity of v-ATPase on the SVs at the ribbon synapse in zebrafish (Einhorn et al., 2012). Rbcn-3a mutant hair cell failed to acidify fully, as reported by lysotracker-DND26 signal intensity ( $\mathrm{pH}$ sensitive dye) and was postulated to be due to fewer assembled v-ATPase. Further, it was suggested to promote holoenzyme assembly as the distribution of $V_{1} A$ subunit was altered in the mutant hair cells. The phenotype was again comparable to zebrafish mutants with defects in v-ATPase subunits (Nuckels et al., 2009). Rbcn3a from rat brain synaptosomal preparation was found to interact with Rbcn-3b (encoded by the gene WDR7) and several sub-units of v-ATPase complex in an IP-MS study (Li et al., 2012). Rbcn-3a was reported to regulate canonical Wnt signalling and endocytosis in neural crest by affecting the endosomal fusion, but not acidification by v-ATPase in a more recent study in zebrafish (Tuttle et al., 2014). Endosomes in Rbcn-3a mutant or v-ATPase $V_{0} a 1$ subunit mutant acidified but remained immature. This suggested that Rbcn-3a might have a cell-type-specific function in the trafficking pathway (Tuttle et al., 2014). 
Interestingly, alteration in Dxml2 levels in humans was found to cause a new complex disorder called polyendocrine-polyneuropathy syndrome (PEPNS) with neurological and endocrine phenotype, defects in gonadotrophic axis, delayed onset of puberty, loss of GnRH neurons in hypothalamus and defect in the GnRH network maturation (Tata et al., 2014, 2017). Mice deficient in Dmxl2 (encoding for Rbcn-3a) were infertile due to the partial GnRH (gonadotropin-releasing hormone) deficiency (Tata et al., 2014). Further, low Rbcn-3a expression in the brain resulted in a defective $\mathrm{GnRH}$ neuron maturation associated to a blunted response to kisspeptin, a hormone that controls $\mathrm{GnRH}$ secretion at puberty. The authors suggested that Rbcn-3a plays a key role in the postnatal maturation and the pubertal reactivation of the $\mathrm{GnRH}$ neuronal network through yet unknown mechanisms (Tata et al., 2017). DMXL2 was also found to be a deafness-associated gene in humans (Chen et al., 2017). Additionally, one of the patient with PEPNS also developed deafness (Tata et al., 2014).

Thus, the reports so far on Rbcn-3a suggests that Rbcn-3a is likely a putative regulator of $\mathrm{v}$ ATPase activity and the consequence of its regulation of v-ATPase activity changes with the cell/tissue type, species complexity and interacting partners. Although Rbcn-3a is enriched in the brain, specifically in synapses and suggested to have a conserved role in v-ATPase regulation through evolution, the protein's function in the fundamental process of SV recycling remains unknown. Acidification process by v-ATPase is fundamental to SV recycling process at the synapse as well as membrane trafficking pathway and studying its regulation, possibly mediated by Rbcn-3a in neurons is essential. Understanding the function of Rbcn-3a at synapses may assist in addressing the question of how an endocytosed vesicle is converted into a mature SV and is the focus of the Chapter 3.2 of this thesis. Also, it could explain the molecular mechanisms behind the phenotypes manifested in patients with altered DMXL2 gene dosage.

\subsection{Aims and experimental approach}

\subsubsection{Chromaffin cell as a model system to study regulated exocytosis}

Release of hormones and neurotransmitters have evolved to be a fast process, as they proceed in millisecond to second range upon arrival of stimulus. Chromaffin cells are the main cell types 
in adrenal medulla, which is the principle site of catecholamine synthesis namely adrenaline and noradrenaline. Chromaffin cells contain large dense-core vesicles (LDCVs), filled with hormones and peptides that are released into the blood stream during a 'fight or a flight' response. Adrenal chromaffin cells are postganglionic sympathetic neurons that are widely used as a model for neurosecretion and neurophysiology. LDCVs originate from the trans-Golgi network and undergo a maturation process before they can fuse with the plasma membrane in a rapid and regulated manner. After the catecholamines are released, an orchestrated process of endocytosis efficiently removes the membrane and proteins of LDCVs and recycles them back to the Golgi. Due the common origin, chromaffin cells and neurons have similar fusion machinery (De Camilli and Jahn, 1990).

Chromaffin cells are a well characterized model system to study the process of calcium regulated release, using similar molecular machines for release as the CNS neurons (Neher, 2006). They allow direct measurement of exocytosis, as well as spatial and temporal control of calcium concentration in the cell to study the basic concept of stimulus dependent release (Rettig and Neher, 2002). Unlike neurons that have a complex morphology, chromaffin cells are small, roundish and so the fusion of LDCVs can be monitored by membrane capacitance measurement. Simultaneously, the cargo released can be measured by combined carbon fiber mediated amperometry recordings. Fast electrophysiological and amperometry recordings together provide the resolution needed to study vesicle release (Figure 9). Further, physiological measurements can be complemented with ultrastructural analysis of number and spatial distribution of the vesicles. They are also suitable for morphological analysis by light microscopy, as well as live imaging. They are especially useful to study the role of particular protein in the release process directly, since exocytosis is not tightly coupled with a local recycling endocytic pathway. 
A

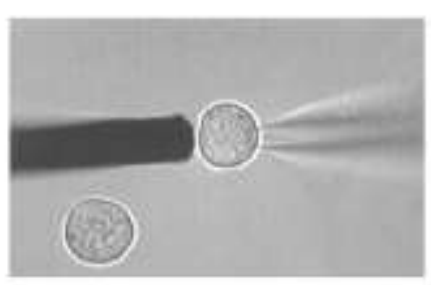

C
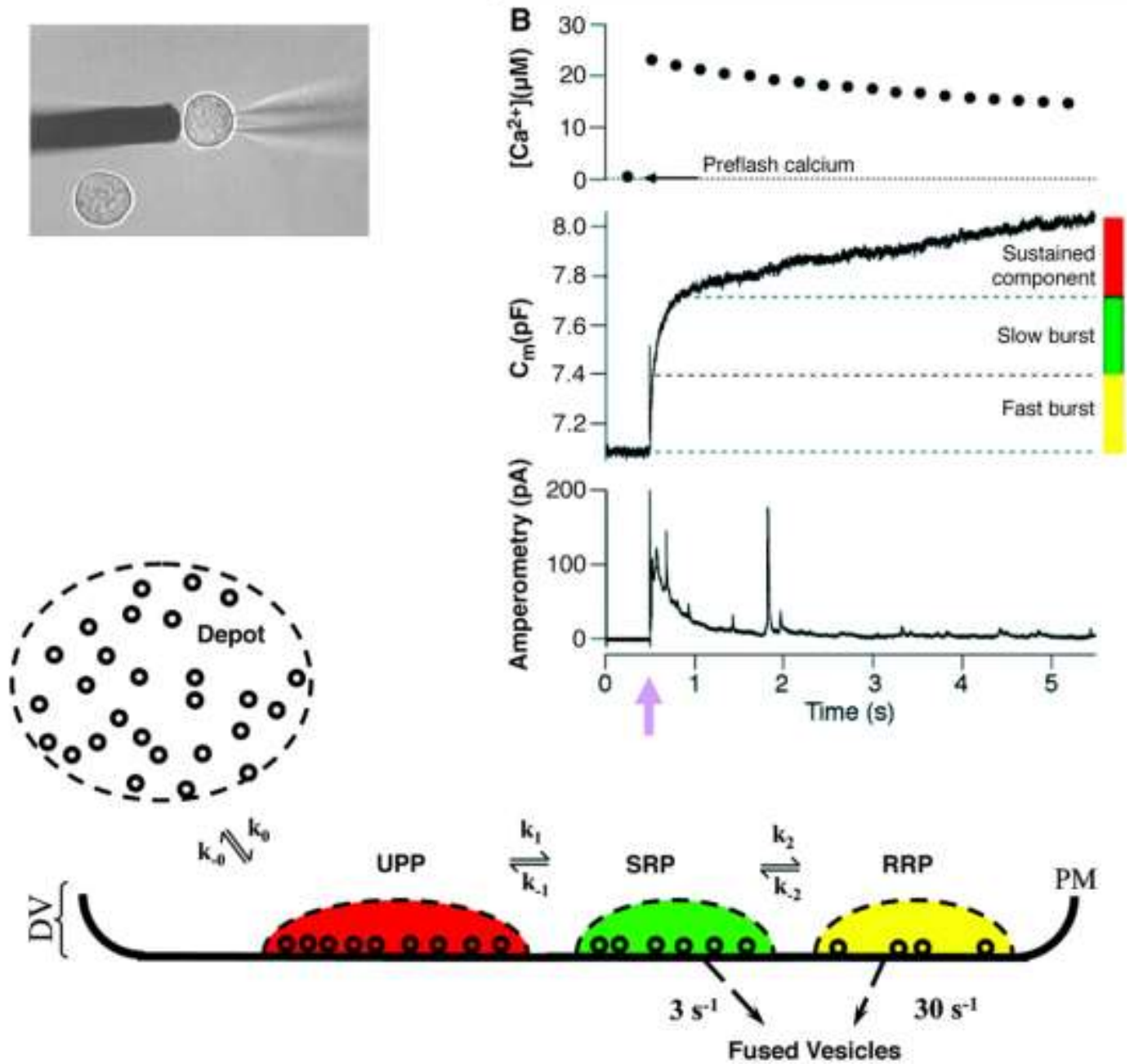

\section{Figure 9 Regulated exocytosis measurements in adrenal chromaffin cells}

(A) Exemplary chromaffin cells, where one of them is being recorded by whole-cell patch clamp as well as being touched by the micro-carbon fiber. (B) Sample response to flash photolysis induced instantaneous increase in calcium to $20 \mu \mathrm{M}$ (upper traces), concomitant changes in membrane capacitance (middle trace) and the amperometric current measured by the carbon fiber electrode (lower panel). The change in capacitance is proportional to the membrane surface area increase caused by the vesicle fusion. (C) The vesicles in the cell are in functionally distinct pool and are depicted by different colors. The primed vesicles are release within $1 \mathrm{~s}$ of increase in $\left[\mathrm{Ca}^{2+}\right]_{i}$ and further divided into slowly releasable pool (SRP) and readily releasable pool (RRP). The vesicles in the depot arrive to the unprimed pool (UPP) when they dock at the membrane. (Adapted from Rettig and Neher, 2002; Neher, 2006). 
All the functional assays in chapter 3.1 were performed on chromaffin cells by flash photolysis and combined capacitance and amperometry traces were recorded. Caged calcium (using nitropheyl-EDTA (NPE)) along with $\mathrm{Ca}^{2+}$ dyes (fura-4F and furaptra) was buffered in the chromaffin cells using a patch pipette. UV flash photolysis resulted in the release of $\mathrm{Ca}^{2+}$ from NPE and instantaneous increase in intracellular calcium levels, which was monitored by the ratiometric fluorescent dyes. This triggers the LDCV fusion with the PM, due to which the membrane surface area increases and is measured by the patching electrode as a change in capacitance. Simultaneously, the amount of catecholamine released is measured using the carbon fiber electrode held at a positive potential of $800 \mathrm{mV}$. The fiber measures the oxidation current from the catecholamine release (Rettig and Neher, 2002).

Ultrastructure analysis of adult chromaffin cell morphology suggested around 1000 vesicles to be in close proximity of the PM (Plattner et al., 1997). However, only a fraction of these vesicles are released after stimulation of the cell (Parsons et al., 1995). Inspection of capacitance traces from flash experiments helps to decipher information about different pools of vesicles released as well as their release kinetics. Release triggered by increase in calcium results in an exocytic burst followed by a sustained phase of secretion (Voets et al., 1999). Burst release component can be resolved further into two kinetically distinct pools, namely the readily releasable pool (RRP) and a slowly releasable pool (SRP) (Voets, 2000). Vesicles are, in general, in four functionally distinct pools (Rettig and Neher, 2002) (Figure 9). Vesicles from the reserve pool enter the unprimed pool (UPP) while they move closer to the PM. UPP vesicles undergo priming, move into the SRP and subsequently into RRP, and thereby releasing the cargo content (but with different time constant, (Figure 9C, Ashery et al., 2000; Voets, 2000)). Additionally, the size of the vesicle pool as well as the rate of transition between them can be regulated by intracellular $\mathrm{Ca}^{2+}$ and proteins involved in exocytosis (Sørensen, 2004). Hence, adrenal chromaffin cell offers a simplified, yet elegant model system to study calcium-regulated exocytosis.

\subsubsection{Aim of my study}

SV recycling is regarded as one of the best controlled process in cell biology and many effectors participating in the process have been identified and their roles characterized. The key steps in 
calcium regulated exocytosis and the machineries controlling the various steps are well described (summarized in (Jahn and Fasshauer, 2012; Südhof, 2013)). Similarly, endocytic machinery and the sequence of events have been well studied (Milosevic, 2018; Saheki and De Camilli, 2012). Additionally, the recycling pathway taken by the cell depending on the activity state is currently under research (Watanabe and Boucrot, 2017). Although the process of exocytosis and endocytosis have been well studied, the intermediate steps between these processes in SV recycling are not yet fully understood. The process of how coupling between the exo- and endocytosis process occurs remains an open question. Moreover, different protein machinery have been studied in the context of specific steps in the SV cycle and their role in other steps of the recycling process at the terminal is not well documented. In the SV cycle, the steps leading to the formation of a fully functional SV after internalization of membrane and cargo are not characterized. Specifically, it is not yet known how and when a newly endocytosed vesicle undergoes acidification, NT refilling and further recruit peripheral SV proteins to be ready for the next round of fusion events. The aim of my doctoral thesis was to address some missing links in the SV recycling process and the specifics are detailed in the next paragraphs.

The mechanism of SV fusion induces endocytosis and how the two processes are coupled the synapse is still being actively investigated. Many endocytic proteins were found in a matrix along with the vesicle clusters during resting state at the terminal (reviewed in (Shupliakov, 2009)). $\mathrm{Ca}^{2+}$ stimulation induced dephosphorylation of several endocytic proteins (e.g. by calceneurin) facilitates their migration to the endocytic zones (Cousin and Robinson, 2001; Murthy and Camilli, 2003). This offers one of a possible mechanism of exo-endocytosis coupling. Additionally, active zone proteins might be implicated in the coupling process (reviewed in (Maritzen and Haucke, 2018)). Intersectin, a SH3 domain-containing protein is implicated in this process as well, since it interacts with exocytic (SNAP25) and endocytosis machinery (endophilin, dynamin, amphiphysin) (Pechstein et al., 2010; Sakaba et al., 2013). Intersectin is suggested to orchestrate the coupling between exo- and endocytosis by shuttling between active and periactive zone (Haucke et al., 2011). Interestingly, endophilin was reported to interact with intersectin-1 through their SH3 domains (Pechstein et al., 2015), but the physiological relevance of this interaction for SV recycling is not fully understood. 
Role of endophilin as a central player in different endocytic pathways is becoming more evident (Milosevic et al., 2018). However, some reports suggest a role beyond endocytosis for these adaptor proteins, in exocytosis and in exo-endocytosis coupling. Interaction of endophilin A1 with VGLUT1 inhibited endophilin-mediated increase in release probability in hippocampal neurons (Vinatier et al., 2006; Weston et al., 2011). In worm's nerve terminal, endophilin was delivered to the site of endocytosis by SV exocytosis and was predominantly targeted to the SV pool at resting state (Bai et al., 2010). This study suggested that the association-dissociation cycle of endophilin with SV parallels exo-and endocytosis cycle at the synapse. However, it is not known if endophilin is targeted to the SV pool in other model organisms. Genetic ablation studies in Drosophila and mice resulted in impaired SV recycling in neurons. The nerve terminals accumulated CCVs, with a concomitant reduction in SVs. Loss of endophilin resulted in impaired synaptic transmission, however the transmission was not abolished (Milosevic et al., 2011; Verstreken et al., 2003). The impaired transmission could be a result of reduction in number of SVs and recycling at the synapse or due to a direct role for endophilin in the SV fusion process itself, or both. It is difficult to address this in the mice terminals, since the process of exocytosis is tightly coupled to the endocytic retrieval mechanisms. To address this question, I used the neurosecretory chromaffin cell system, where exocytosis is not tightly coupled with the recycling endocytic pathways. To test if endophilins have a direct role in secretory vesicle, I used fast electrophysiological and electrochemical techniques, and the results are presented in Chapter 3.1.

The other missing link in the SV recycling process is how and when a newly formed vesicle by endocytosis undergoes acidification, NT refilling and recruit peripheral SV proteins, resulting in the formation of a fully functional mature SV. Studies on spatial and temporal regulation of SV acidification have been challenged by the limitations of tools and model system with the required resolution. v-ATPase is engaged in a diverse spectrum of processes like endocytosis, NT refilling, degration of macromolecules, maintaining homeostatis of the cytosol, among many others. $v$ ATPase is central to the organelle acidification in various cellular processes; however, deciphering the functional consequence of v-ATPase is challenging since genetic perturbation of the complex is lethal or has severe consequence to the model organism (Beyenbach and Wieczorek, 2006). Understanding the regulation of v-ATPase function in general is therefore essential, also in the context of SV recycling. The available reports suggest a few possibilities 
including regulation by assembly/disassembly cycle (Bodzęta et al., 2017), intravesicular pH (Poëa-Guyon et al., 2013), Clathrin coat (Farsi et al., 2018) and accessory factors.

Several reports have suggested that Rbcn-3a is likely a putative regulator of v-ATPase activity. The consequence of its regulation of v-ATPase activity changes with the cell/tissue type, species complexity and interacting partners. Although the initial reports showed that they are enriched in the brain, the function of this protein in SV recycling remains unknown in mammalian neuronal synapses. It was suggested to function as an adaptor that mediated the Rab3 cycle through interaction with Rab3-GEF and GAP (Kawabe et al., 2003; Nagano et al., 2002). However, the follow up studies were challenged by the lack of tools to characterize the function of this novel, but evolutionarily conserved protein, Rabconnectin-3a. In a search for a potential v-ATPase regulator in the context of SV recycling, we chose Rbcn-3a as a likely candidate. Understanding the function of Rbcn-3a at synapses may hold a key in addressing the question of how an endocytosed vesicle is converted into a mature SV as well as explain the molecular mechanisms behind the phenotypes manifested in patients with altered DMXL2 gene dosage. I have presented the findings from WT and Rbcn-3a KO studies in hippocampal neurons and how it could act as a link between endocytosis, re-acidification and SV maturation in Chapter 3.2.

In sum, my two main aims were:

1) Characterizing the role of endophilins in chromaffin cell exocytosis and endocytosis (results are presented in Chapter 3.1)

2) Studying the role of rabconnectin-3a in SV recycling process at the mammalian synapse (results are presented in Chapter 3.2) 


\section{Materials}

\subsection{Chemicals and reagents}

Chemical used in this study are listed below in Table 1.

Table 1 - List of chemicals, their supplier and catalog number

\begin{tabular}{|l|l|l|}
\hline Chemical & Supplier & Catalog number \\
\hline 2-Mercaptoethanol & Carl Roth & 4227.3 \\
\hline 4',6-Diamidino-2-phenylindole (DAPI) & Carl Roth & 6335.1 \\
\hline Acetic acid $\left(\mathrm{CH}_{3} \mathrm{COOH}\right)$ & Carl Roth & X895.2 \\
\hline Acrylamide & Carl Roth & 3029.2 \\
\hline Agarose & Biozym & 840004 \\
\hline Ammonium chloride $\left(\mathrm{NH}_{4} \mathrm{Cl}\right)$ & Carl Roth & K298.1 \\
\hline Ammonium Persulfate (APS) & AMRESCO & 486 \\
\hline Bafilomycin A1 & InvivoGen & trl-baf1 \\
\hline Bicuculline & Sigma-Aldrich & 14340 \\
\hline Bovine serum albumin (BSA) & Carl Roth & CP84.2 \\
\hline Bromophenol blue & Carl Roth & T116.1 \\
\hline Calcium chloride $($ CaCl $)$ & Carl Roth & CN93.1 \\
\hline Gelatin from cold water fish & Sigma-Aldrich & G7765 \\
\hline $\begin{array}{l}\text { cOmplete ULTRA Tablets, EDTA-free, } \text { Protease } \\
\text { Inhibitor Cocktail }\end{array}$ & Sigma-Aldrich & 5892953001 \\
\hline Control peptide (VAMP2) & & \\
\hline Creatine phosphate & SySy & $104-2 \mathrm{P}$ \\
\hline d-AP5 & Sigma-Aldrich & 10127566001 \\
\hline Dithiothreitol (DTT) & Tocris & 0106 \\
\hline EGTA & Carl Roth & 6908.1 \\
\hline Ethanol absolute & Carl Roth & 3054.1 \\
\hline Ethidium bromide & VWR & $20,816,298$ \\
\hline Ethylenediaminetetraacetic acid (EDTA) & VWR & E406 \\
\hline FM 1-43 & Carl Roth & 8043.2 \\
\hline Fura-4F & Molecular Probes & T-35356 \\
\hline Furaptra & Invitrogen & F14174 \\
\hline Glucose & Invitrogen & M1290 \\
\hline Glutaraldehyde & Sigma-Aldrich & G8270 \\
\hline Glycerol & ApplieChem & A3166,0100 \\
\hline Glycine & Carl Roth & 7530.1 \\
\hline Goat Serum & Carl Roth & 3908.3 \\
\hline & Life Technologies & $10000 C$ \\
\hline & & \\
\hline
\end{tabular}




\begin{tabular}{|c|c|c|}
\hline $\begin{array}{l}\text { Halt'TM Protease \& Phosphatase single use Inhibitor } \\
\text { Cocktail (100X) }\end{array}$ & Thermo Fisher & 78442 \\
\hline Hellmanex-II & Hellma Analytics & RV 800237 \\
\hline HEPES & Carl Roth & HN77.2 \\
\hline LuminataTM Forte Western HRP Substrate & Millipore & WBLUF0500 \\
\hline Magnesium chloride $\left(\mathrm{MgCl}_{2}\right)$ & VWR & 8.14733 .0100 \\
\hline Methanol & VWR & 20903.368 \\
\hline mCLING-Atto 647 & SySy & $710006 \mathrm{AT} 1$ \\
\hline Mg-Adenosine triphosphate (ATP) & Sigma-Aldrich & A9187 \\
\hline Mowiol $^{\circledR} 4-88$ & AppliChem & A9011 \\
\hline Na-GTP & Sigma-Aldrich & G8877 \\
\hline $\mathrm{NaHCO}_{3}$ & Carl Roth & P029.3 \\
\hline $\mathrm{N}$-dodecylmaltoside & Carl Roth & CN26.2 \\
\hline Nitrophenyl-EGTA (NPE) & ThermoFischer & N6802 \\
\hline Pepstatin A & Sigma-Aldrich & P4265 \\
\hline Phospocreatine kinase & Roche & 10127566001 \\
\hline PhosStop tablets & Sigma-Aldrich & 4906837001 \\
\hline PMSF (phenymethylsulfonyl fluoride) & AppliChem & A0999 \\
\hline Poly-L-Lysine & Sigma-Aldrich & P-8920 \\
\hline Potassium chloride $(\mathrm{KCl})$ & Volu-Sol & 83608.26 \\
\hline Potassium dihydrogen phosphate $\left(\mathrm{KH}_{2} \mathrm{PO}_{4}\right)$ & Carl Roth & 3904.1 \\
\hline Potassium phosphate dibasic $\left(\mathrm{K}_{2} \mathrm{HPO}_{4}\right)$ & Carl Roth & P749.2 \\
\hline Proteinase $\mathrm{K}$ & Roche & 3115879001 \\
\hline Skim Milk Powder & Fluka & 70166 \\
\hline Sodium cacodylate & Carl Roth & 5169.1 \\
\hline Sodium chloride $(\mathrm{NaCl})$ & AppliChem & A1430,0010 \\
\hline Sodium deoxycholate & Sigma-Aldrich & 30970 \\
\hline Sodium hydroxide pellets $(\mathrm{NaOH})$ & Carl Roth & 6771.1 \\
\hline Sodium dodecyl sulfate (SDS) & Sigma-Aldrich & L4509-500G \\
\hline Strychnine & Sigma-Aldrich & S0532 \\
\hline Tetrodotoxin (TTX) & Tocris & $T-550$ \\
\hline Tetramethylethylenediamine (TEMED) & Sigma-Aldrich & T7024 \\
\hline Transferrin-Alexa546 & $\begin{array}{l}\text { ThermoFischer } \\
\text { Scientific }\end{array}$ & T133443 \\
\hline Tris(hydroxymethyl)aminomethane (Tris)-Base & Sigma-Aldrich & T1503 \\
\hline Tris(hydroxymethyl)aminomethane (Tris)-HCl & Carl Roth & 9090.2 \\
\hline Tween-20 & AMRESCO & 0777-1L \\
\hline VF2.1.Cl & \multicolumn{2}{|c|}{$\begin{array}{l}\text { Max-Planck institute for Biophysical } \\
\text { Chemistry }\end{array}$} \\
\hline Vitamin C & Sigma-Aldrich & A5960 \\
\hline
\end{tabular}




\subsection{Solutions}

The various buffers and solutions used in the study are listed in Table 2.

Table 2 - List of buffers and solutions used in this study

\begin{tabular}{|c|c|}
\hline Solution & Recipe \\
\hline $\begin{array}{l}\text { 4\% paraformaldehyde } \\
\text { (PFA) }\end{array}$ & $\begin{array}{l}\text { 4\% PFA in PBS, pH 7.4; heat PBS to } 58^{\circ} \mathrm{C} \text {, add sodium hydroxide } \\
\text { drop wise until PFA dissolves }\end{array}$ \\
\hline $5 \%$ milk & $5 \%(w / v)$ skim milk powder in TBS-T \\
\hline Acid wash buffer & $\begin{array}{l}\text { Modified tyrode's buffer with 2-(N-morpholino)ethanesulfonic acid } \\
\text { (MES) to pH5.5 }\end{array}$ \\
\hline $\begin{array}{l}\text { Adrenal } \\
\text { solution }\end{array}$ & $\begin{array}{l}\text { DMEM supplemented with } 0.2 \mathrm{mg} / \mathrm{mL} \text { L-cysteine, } 0.5 \mathrm{mM} \text { EDTA, } 1 \\
\mathrm{mM} \mathrm{CaCl} \text {, and } 20-25 \mathrm{U} / \mathrm{mL} \text { papain (Worthington Biochemical) } \\
\text { (equibrated in } 8 \% \mathrm{CO}_{2} \text { ) }\end{array}$ \\
\hline Ammonium buffer & Tyrode buffer modified to contain $50 \mathrm{mM} \mathrm{NH}_{4} \mathrm{Cl}$ and $69 \mathrm{mM} \mathrm{NaCl}$ \\
\hline neutralization buffer & $50 \mathrm{mM} \mathrm{NH}_{4} \mathrm{Cl}$ in PBS \\
\hline $\begin{array}{l}\text { Blocking } \\
\text { (stainings) }\end{array}$ & $3 \%(w / v)$ BSA, $0.2 \%(w / v)$ cold fish gelatin, $1 \%$ goat serum in PBS \\
\hline Cell lysis buffer & $\begin{array}{l}1.5 \% \mathrm{~N} \text {-dodecyl } \beta \text {-D-maltoside in PBS supplemented with } 1 \mathrm{X} \\
\text { protease and phosphatase inhibitor }\end{array}$ \\
\hline $\begin{array}{l}\text { Chromaffin cell culture } \\
\text { medium }\end{array}$ & $\begin{array}{l}\text { DMEM supplemented with } 4 \mu \mathrm{L} / \mathrm{mL} \text { penicillin/streptomycin (Invitrogen) } \\
\text { and } 10 \mu \mathrm{L} / \mathrm{mL} \text { insulin-transferrin-selenium-X (Invitrogen). }\end{array}$ \\
\hline $\begin{array}{lr}\text { Dulbecco } & \text { Modified } \\
\text { Eagle's } & \text { Medium } \\
\text { (DMEM) } & \\
\end{array}$ & $\begin{array}{l}\text { (DMEM) medium containing } 10 \% \text { fetal bovine serum (FBS) and } 1 \% \\
\text { penicillin/streptomycin }\end{array}$ \\
\hline EM fixative & $1.3 \%$ glutaraldehyde prepared in $66 \mathrm{mM}$ sodium cacodylate buffer \\
\hline $\begin{array}{l}\text { extracellular } \\
\text { solution(chromaffin } \\
\text { cells) }\end{array}$ & $\begin{array}{l}145 \mathrm{mM} \mathrm{NaCl}, 2.8 \mathrm{mM} \mathrm{KCl}, 2 \mathrm{mM} \mathrm{CaCl} \\
2,1 \mathrm{mM} \mathrm{MgCl}_{2}, 10 \mathrm{mM} \mathrm{HEPES} \text {, } \\
\text { and } 2 \mathrm{mg} / \mathrm{mL} \text { D-glucose, pH 7.2, } \sim 305 \mathrm{mOsM} / \mathrm{kg}\end{array}$ \\
\hline HBSS medium & $\begin{array}{l}\text { 1X Hank's Balanced Salt solution/HBSS, } 1 \mathrm{mM} \text { HEPES pH } 7.4,0.5 \% \\
\text { penicillin/streptomycin, } 100 \mathrm{mM} \text { pyruvic acid, } \mathrm{H}_{2} \mathrm{O}\end{array}$ \\
\hline High $\mathrm{K}^{+}$buffer & $\begin{array}{l}88 \mathrm{mM} \mathrm{NaCl}, 59 \mathrm{mM} \mathrm{KCl}, 2 \mathrm{mM} \mathrm{CaCl} \\
\text { and } 2 \mathrm{mg} / \mathrm{ml} \text { D-glucose, } \mathrm{pH} \text { 7.20, } 300 \mathrm{mOsM} / \mathrm{kg}\end{array}$ \\
\hline $\begin{array}{l}\text { Homogenization } \\
\text { buffer }\end{array}$ & $\begin{array}{l}2 \mathrm{M} \mathrm{NaCl}, 0.5 \mathrm{M} \text { EDTA, } 1 \mathrm{M} \mathrm{HEPES} \text { in } \mathrm{dH}_{2} \mathrm{O} \mathrm{pH} 7.4 \text { add } 1 \text { phospho- } \\
\text { stop tablet/10 mL, } 1 \text { protease inhibitor tablet } / 50 \mathrm{~mL} \text { and } 1 \mathrm{mM} \text { DTT for } \\
\text { activation }\end{array}$ \\
\hline $\begin{array}{l}\text { Homogenization } \\
\text { buffer for vesicle } \\
\text { isolation }\end{array}$ & $\begin{array}{l}320 \mathrm{mM} \text { Sucrose, } 5 \mathrm{mM} \text { HEPES (pH 7.4), supplemented with } 200 \mu \mathrm{M} \\
\text { PMSF (phenymethylsulfonyl fluoride) and } 1 \mu \mathrm{g} / \mathrm{mL} \text { pepstatin-A }\end{array}$ \\
\hline $\begin{array}{l}\text { Imaging buffer (non- } \\
\text { neuronal cells) }\end{array}$ & $\begin{array}{l}136 \mathrm{mM} \mathrm{NaCl}, 2.5 \mathrm{mM} \mathrm{KCL}, 2 \mathrm{mM} \mathrm{CaCl}, 1 \mathrm{mM} \mathrm{MgCl}_{2}, 10 \mathrm{mM} \\
\text { HEPES, in } \mathrm{dH}_{2} \mathrm{O}, \mathrm{pH} 7.4,300 \mathrm{mOsM} / \mathrm{kg}\end{array}$ \\
\hline
\end{tabular}




\begin{tabular}{|c|c|}
\hline Inactivation solution & $\begin{array}{l}\text { DMEM supplemented with } 10 \% \text { heat-inactivated fetal calf serum } \\
\text { (Invitrogen), } 2.5 \mathrm{mg} / \mathrm{mL} \text { albumin and } 2.5 \mathrm{mg} / \mathrm{mL} \text { trypsin inhibitor }\end{array}$ \\
\hline $\begin{array}{l}\text { Intracellular solution } \\
\text { (flash experiments) }\end{array}$ & $\begin{array}{l}100 \mathrm{mM} \text { Cs-glutamate, } 8 \mathrm{mM} \mathrm{NaCl}, 4 \mathrm{mM} \mathrm{CaCl} \text {, } 32 \mathrm{mM} \text { Cs-HEPES, } \\
2 \mathrm{mM} \text { Mg-ATP, } 0.3 \mathrm{mM} \mathrm{GTP}, 5 \mathrm{mM} \mathrm{NPE}, 0.4 \mathrm{mM} \text { Fura-4F, } 0.4 \mathrm{mM} \\
\text { Furaptra, and } 1 \mathrm{mM} \text { vitamin C, pH } 7.2 \text { (osmolarity adjusted to } \sim 295 \\
\text { mOsm) }\end{array}$ \\
\hline $\begin{array}{lr}\text { Intracellular } & \text { solution } \\
\text { (single } & \text { spike } \\
\text { amperometry) } & \end{array}$ & $\begin{array}{l}70 \mathrm{mM} \text { Cs-glutamate, } 8 \mathrm{mM} \mathrm{NaCl}, 4 \mathrm{mM} \mathrm{CaCl}, 22.5 \mathrm{mM} \text { Cs-HEPES, } \\
2 \mathrm{mM} \text { Mg-ATP, } 0.3 \mathrm{mM} \mathrm{Na}-\mathrm{GTP}, 37 \mathrm{mM} \mathrm{Ca} \mathrm{Ca}_{2+} \text { DPTA, } 0.32 \mathrm{mM} \text { Fura- } \\
\text { 4F and } 0.48 \mathrm{mM} \text { Furaptra pH } 7.2 \text { (osmolarity adjusted to } \sim 300 \text { mOsm) }\end{array}$ \\
\hline K-gluconate buffer & $100 \mathrm{mM}$ K-gluconate, $10 \mathrm{mM}$ MOPS (pH 7.4), $4 \mathrm{mM} \mathrm{MgSO}_{4}(\mathrm{pH} 7.4)$ \\
\hline Locke's solution & $\begin{array}{l}1,0.85 \mathrm{mM} \mathrm{NaH}_{2} \mathrm{PO}_{4}, 2.15 \mathrm{mM} \mathrm{Na}_{2} \mathrm{HPO}_{4} \text {, } \\
2\end{array}$ \\
\hline $\begin{array}{l}88 \circledast \\
\text { medium }\end{array}$ & $\begin{array}{l}3.6 \mathrm{M} \text { glycerol, } 3 \mathrm{M} \text { Mowiol } 4-88,133 \mathrm{mM} \text { Tris-Base in } \mathrm{dH}_{2} \mathrm{O} \text {. Stir for } \\
\text { several hours at } 45^{\circ} \mathrm{C} \text {, centrifuge at } 5000 \mathrm{~g} \text { for } 15 \mathrm{~min} \text {, collect } \\
\text { supernatant, store at }-20^{\circ} \mathrm{C}\end{array}$ \\
\hline $\begin{array}{l}\text { Neuronal } \\
\text { medium }\end{array}$ & $\begin{array}{l}\text { Neurobasal medium with } 1 \% \text { glutamax, } 2 \% \text { supplement B27, } 0.5 \% \\
\text { penicillin/streptomycin }\end{array}$ \\
\hline $\begin{array}{l}\text { Neuronal enzyme } \\
\text { solution }\end{array}$ & $\begin{array}{l}\mathrm{mM} \text { cysteine, } 20 \mathrm{U} / \mathrm{mL} \text { papain, } 0.75 \mathrm{mM} \text { EDTA, } \\
0 \mu \mathrm{l} / \mathrm{ml} \text { DNase }\end{array}$ \\
\hline $\begin{array}{l}\text { Neuronal extracellular } \\
\text { solution }\end{array}$ & $\begin{array}{l}2 \mathrm{mM} \mathrm{MgCl}_{2}, 2 \mathrm{mM} \mathrm{CaCl}_{2}, 14 \mathrm{mM} \text { glucose, } \\
\text {-310 mOsm). Drugs } 20 \mu \mathrm{M} \text { bicuculline, } 2 \\
\text { and } 50 \mu \mathrm{M} \text { d-AP5 were added to isolate }\end{array}$ \\
\hline $\begin{array}{l}\text { Neuronal intrapipette } \\
\text { solution }\end{array}$ & $\begin{array}{l}\text { eatine phosphate, } 4 \mathrm{mM} \mathrm{Na}- \\
\text { dd } 50 \mathrm{U} / \mathrm{mL} \text { phospocreatine }\end{array}$ \\
\hline Neuronal lysis buffer & $\begin{array}{l}\text { 1Tris } \mathrm{pH} 7.4,150 \mathrm{mM} \mathrm{NaCl}, 1 \mathrm{mM} \text { EDTA } \\
1 \mathrm{X} \text { protease and phosphatase inhibitor }\end{array}$ \\
\hline $\begin{array}{l}\text { Neuronal plating } \\
\text { medium }\end{array}$ & $\begin{array}{l}\text { erum, } 1 \% \text { glutamax, } 2 \% \\
\text { n }\end{array}$ \\
\hline abilization & $\begin{array}{l}3 \%(\mathrm{w} / \mathrm{v}) \mathrm{BSA}, 0.2 \%(\mathrm{w} / \mathrm{v}) \text { cold fish gelatin, } 1 \% \text { goat serum, } 0.1 \%(\mathrm{v} / \mathrm{v}) \\
\text { Triton-X100 in PBS }\end{array}$ \\
\hline $\begin{array}{l}\text { te buffered } \\
\text { BS) }\end{array}$ & $\begin{array}{l}137 \mathrm{mM} \mathrm{NaCl}, 2.7 \mathrm{mM} \mathrm{KCl}, 10 \mathrm{mM} \mathrm{Na}_{2} \mathrm{HPO}_{4} \times 2 \mathrm{H}_{2} \mathrm{O}, 2 \mathrm{mM} \mathrm{KH}_{2} \mathrm{PO}_{4} \\
\text { in } \mathrm{dH}_{2} \mathrm{O} \text {, p.H. } 7.4 \text {, autoclave }\end{array}$ \\
\hline RIPA buffer & $\begin{array}{l}50 \mathrm{mM} \text { Tris } \mathrm{pH} 8.0,150 \mathrm{mM} \mathrm{NaCl}, 1 \% \text { Triton } \mathrm{X}-100,0.1 \% \text { SDS and } \\
0.5 \% \text { Sodium deoxycholate with fresh } 1 \mathrm{X} \text { protease/phosphatase } \\
\text { inhibitor supplement }\end{array}$ \\
\hline & 0.96 M Glycine, $0.5 \%$ (w/v) SDS \\
\hline SDS loading buffer & $\begin{array}{l}62.5 \mathrm{mM} \text { Tris, } \mathrm{pH} 6.8,50 \%(\mathrm{v} / \mathrm{v}) \text { Glycerol, } 12 \%(\mathrm{w} / \mathrm{v}) \text {, SDS, } 0.06 \% \\
\text { (w/v) Bromophenol blue, (Add 5\% 2-Mercaptoetanol freshly prior to } \\
\text { use) }\end{array}$ \\
\hline SNET buffer & $\begin{array}{l}20 \mathrm{mM} \text { Tris (pH 8.0), } 5 \mathrm{mM} \text { EDTA (pH 8.0), } 400 \mathrm{mM} \mathrm{NaCl}, 0.5 \%(\mathrm{w} / \mathrm{v}) \\
\text { SDS in } \mathrm{dH}_{2} \mathrm{O}\end{array}$ \\
\hline & $\mathrm{OOH}, 1 \mathrm{ml}$ \\
\hline TBS-T & 1 \\
\hline
\end{tabular}




\begin{tabular}{|c|c|}
\hline TE buffer & $10 \mathrm{mM}$ Tris-HCl, $0.2 \mathrm{mM}$ EDTA in $\mathrm{dH}_{2} \mathrm{O}, \mathrm{pH} 7.5$ \\
\hline Transfer Buffer & $25 \mathrm{mM}$ Tris-Base, $0.2 \mathrm{M}$ Glycine, $20 \%$ (v/v) Methanol in $\mathrm{dH}_{2} \mathrm{O}$ \\
\hline $\begin{array}{l}\text { Tris buffered saline } \\
\text { (TBS) }\end{array}$ & $20 \mathrm{mM}$ Tris-Base, $150 \mathrm{mM} \mathrm{NaCl}$ in $\mathrm{dH}_{2} \mathrm{O}, \mathrm{pH} 7.6$ \\
\hline Tyrode's buffer & $\begin{array}{l}119 \mathrm{mM} \mathrm{NaCl}, 5 \mathrm{mM} \mathrm{KCl}, 25 \mathrm{mM} \text { HEPES buffer, } 2 \mathrm{mM} \mathrm{CaCl}_{2}, 2 \mathrm{mM} \\
\mathrm{MgCl}_{2}, 6 \mathrm{~g} / \mathrm{L} \text { glucose, } \mathrm{pH} 7.4 \text { with NaOH }(50 \mu \mathrm{M} \text { d-AP5 })\end{array}$ \\
\hline
\end{tabular}

\subsection{Consumables and kits}

The kits and consumables used are listed in Table 3.

Table 3 - Kits and consumable products used in this study

\begin{tabular}{|l|l|l|}
\hline Product & Supplier & Catalog number \\
\hline $\begin{array}{l}\text { Amicon Ultra-1PLHK Ultracel- } \\
\text { PL100kDa (15ml) }\end{array}$ & $\begin{array}{l}\text { EMD } \\
\text { MilliporeTM }\end{array}$ & UFC910096 \\
\hline 12-well TC plate & CytoOne & CC7682-7512 \\
\hline 6-well TC plate & CytoOne & CC7682-7506 \\
\hline 96-well TC plate & CytoOne & CC7682-7596 \\
\hline Cell scraper & StarLab & CC7600-0202 \\
\hline Coverslips $18 \mathrm{~mm} \mathrm{(1.5H} \mathrm{thickness)}$ & Marienfeld & 17580 \\
\hline Cryogenic vial 2 mL & Fisher Brand & 1050026 \\
\hline Cuvettes polystyrol/polystyrene & Sarstedt & $67-742$ \\
\hline Eppendorf tubes (1.5 mL) & StarLab & E1415-1500 \\
\hline Eppendorf tubes (2 mL) & StarLab & S1620-2700 \\
\hline G-coupled magnetic beads & Invitrogen & $10007 \mathrm{D}$ \\
\hline GFP-Trap®_A & Chromtek & gta-10 \\
\hline GoTaq Flexi DNA polymerase kit & Promega & M8291 \\
\hline iScrip cDNA Synthesis Kit & Bio-Rad & $170-8891$ \\
\hline $\begin{array}{l}\text { iTaqTM Universal SYBR Green } \\
\text { Supermix }\end{array}$ & Bio-Rad & $172-5124$ \\
\hline Microscope slides & Carl Carl Roth & 2111 \\
\hline Needles 24G & B.Braun & 4657675 \\
\hline Needles 27G & B.Braun & 4657705 \\
\hline Nitrocellulose membrane & BIO-Rad & 1620115 \\
\hline $\begin{array}{l}\text { PageRuler Plus Prestained Protein } \\
\text { Ladder }\end{array}$ & Thermo & 26619 \\
\hline Pasteur pipet plastic & Scientific & \\
\hline $\begin{array}{l}\text { PCR plate 384-well skirted ABI-Type } \\
\text { (Universal) }\end{array}$ & Carl Carl Roth & EA63.1 \\
\hline Petri dish 10 cm & Etab & E1042-3840 \\
\hline Petri dish 2 cm & Sarstedt & 82.1473 .001 \\
\hline & Sarstedt & 82.1135 .500 \\
\hline
\end{tabular}




\begin{tabular}{|l|l|l|}
\hline Pierce BCA Protein Assay kit & $\begin{array}{l}\text { Thermo } \\
\text { Scientific }\end{array}$ & 23225 \\
\hline Super frost slides & Th. Geyer & 7695019 \\
\hline Syringe $1 \mathrm{~mL}$ & VWR & $613-2040$ \\
\hline Tips $10 \mu \mathrm{L}$ TipOne & StarLab & $\mathrm{S} 111-3210$ \\
\hline Tips $1000 \mu \mathrm{L}$ TipOne & StarLab & $\mathrm{S} 1111-6001$ \\
\hline Tips $20 \mu \mathrm{L}$ TipOne & StarLab & $\mathrm{S} 120-1810$ \\
\hline Tips $200 \mu \mathrm{L}$ TipOne & StarLab & $\mathrm{S} 1120-8800$ \\
\hline Tubes $15 \mathrm{~mL}$ & Sarstedt & 62.554 .502 \\
\hline Tubes $50 \mathrm{~mL}$ & Corning & 430829 \\
\hline Whatman Western Blot Paper & TH-Geyer & $4-01-60-0041$ \\
\hline
\end{tabular}

\subsubsection{Cell culture material}

The materials and equipment specifically used for cell culture experiments are listed in Table 4.

Table 4 - List of cell culture materials

\begin{tabular}{|l|l|l|}
\hline Product & Supplier & $\begin{array}{l}\text { Catalog } \\
\text { number }\end{array}$ \\
\hline Cryogenic vial 2 mL & Fisher Brand & 1050026 \\
\hline B27 Supplement (50X) serum free 10ml & Gibco® (Life Technologies) & $17504-044$ \\
\hline Dimethyl sulfoxide (DMSO) & Sigma-Aldrich & $41639-100 \mathrm{ML}$ \\
\hline DMEM powder & Gibco® (Life Technologies) & 52100 \\
\hline DMEM, high glucose, pyruvate & ThermoFisher Scientific & $41966-052$ \\
\hline $\begin{array}{l}\text { Fetal Bovine Serum, qualified, heat } \\
\text { inactivated }\end{array}$ & ThermoFisher Scientific & 10500064 \\
\hline Geneticin & Gibco® (Life Technologies) & 11811031 \\
\hline Glutamax & Gibco® (Life Technologies) & $35050-061$ \\
\hline $\begin{array}{l}\text { Hanks balanced salt solution (HBSS- } \\
\text { Buffer) }\end{array}$ & Gibco® (Life Technologies) & $14170-088$ \\
\hline Incubator HERA cell 240 & ThermoFisher Scientific & 51026331 \\
\hline $\begin{array}{l}\text { Insulin-Transferrin-Selenium- } \\
\text { Ethanolamine (ITS -X) (100X) }\end{array}$ & Gibco® (Life Technologies) & 51500056 \\
\hline L-cysteine & Sigma-Aldrich & C7352 \\
\hline Lipofectamine 2000 Reagent & ThermoFisher Scientific & $11668-019$ \\
\hline Neubauer counting chamber & VWR & $631-0696$ \\
\hline Neurobasal medium & Gibco® (Life Technologies) & $21103-045$ \\
\hline Nucleofactor X Core Unit & Lonza & AAT-1002B/X \\
\hline Opti-MEM® I Reduced Serum Medium & Gibco® (Life Technologies) & $31985-047$ \\
\hline
\end{tabular}




\begin{tabular}{|c|c|c|}
\hline Papain & Worthington Biochemical & LS003127 \\
\hline $\begin{array}{l}\text { P3 Primary Cell 4D-Nucleofector }{ }^{\circledR} \text { X Kit } \\
\text { S (32 RCT) }\end{array}$ & Lonza & V4XP-3024 \\
\hline $\begin{array}{l}\text { P4 Primary Cell 4D-Nucleofector }{ }^{\circledR} \text { X Kit } \\
\text { S (32 RCT) }\end{array}$ & Lonza & V4XP-4032 \\
\hline PBS tablets & VWR & 97062-732 \\
\hline Penicillin-Streptomycin $(10,000 \mathrm{U} / \mathrm{mL})$ & ThermoFisher Scientific & 15140122 \\
\hline Petri dish $10 \mathrm{~cm}$ & Sarstedt & 83.3902 .300 \\
\hline Sodium pyruvate & Gibco® (Life Technologies) & $1560-039$ \\
\hline Trypsin-EDTA $(0.05 \%)$, phenol red & Gibco® (Life Technologies) & 11580626 \\
\hline
\end{tabular}

\subsection{Antibodies}

All antibodies used for immunocytochemistry and western blot analysis are listed in Table 5 -8.

Table 5- Primary antibodies used for immunohistochemistry

\begin{tabular}{|l|l|l|l|}
\hline Target protein & Manufacturer & species & concentration \\
\hline bassoon & Enzo SAP7F407 & mouse & $1: 400$ \\
\hline Chromogranin-A & Abcam ab45138 & rabbit & $1: 3000$ \\
\hline endophilin A1 & SySy (lot \#664) & $\begin{array}{l}\text { guinea } \\
\text { pig }\end{array}$ & $1: 1000$ \\
\hline endophilin A2 & Santa Cruz SH3GL1 & mouse & $1: 500$ \\
\hline endophilin A2 & SySy (lot \#8407) & rabbit & $1: 500$ \\
\hline homer 1 & SySy 160002 & rabbit & $1: 250$ \\
\hline intersectin-1 & gift of Prof.Haucke, Berlin & rabbit & $1: 500$ \\
\hline intersectin-2 & Sigma-Aldrich HPA036475 & rabbit & $1: 500$ \\
\hline MAP2 & Abcam AB5392 & chicken & $1: 1000$ \\
\hline Munc18-1 & SySy 116002 & rabbit & $1: 500$ \\
\hline rab3 & SySy Cl 42.1 & mouse & $1: 250$ \\
\hline rabconnectin-3a & Sigma-Aldrich HPA039375 & rabbit & $1: 250$ \\
\hline SNAP25 & SySy 116002 & rabbit & $1: 500$ \\
\hline Synaptobrevin-2 (VAMP2) & SySy Cl69.1 & mouse & $1: 1000$ \\
\hline synaptophysin & SySy Cl G96 & rabbit & $1: 500$ \\
\hline synaptotagmin-1 & SySy 105011 & Mouse & $1: 400$ \\
\hline synaptotagmin-7 & SySy 105173 & rabbit & $1: 500$ \\
\hline syntaxin & SySy 110011 & rabbit & $1: 500$ \\
\hline VGLUT1 & Abcam AB5905 & $\begin{array}{l}\text { guinea } \\
\text { pig }\end{array}$ & $1: 1000$ \\
\hline
\end{tabular}


Table 6 - Secondary antibodies used for immunohistochemistry

\begin{tabular}{|l|l|l|l|l|}
\hline Target species & Fluorophore & Manufacturer & species & concentration \\
\hline Chicken & Alexa405 & Abcam AB175674 & Goat & $1: 1000$ \\
\hline Guinea Pig & Alexa488 & Life Technologies & Goat & $1: 200$ \\
\hline Guinea Pig & Alexa568 & Life Technologies & Goat & $1: 200$ \\
\hline Mouse & Alexa488 & Life Technologies & Goat & $1: 200$ \\
\hline Mouse & Alexa568 & Life Technologies & Goat & $1: 200$ \\
\hline Mouse & Alexa594 & Life Technologies & Goat & $1: 200$ \\
\hline Mouse & Alexa647 & Life Technologies & Goat & $1: 200$ \\
\hline Rabbit & Alexa488 & Life Technologies & Goat & $1: 200$ \\
\hline Rabbit & Alexa647 & Life Technologies & Goat & $1: 200$ \\
\hline Rabbit & Alexa568 & Life Technologies & Goat & $1: 200$ \\
\hline Rabbit & Alexa594 & Life Technologies & Goat & $1: 200$ \\
\hline
\end{tabular}

Table 7 - Primary antibodies used for western blot analysis

\begin{tabular}{|l|l|l|l|}
\hline Target protein & Manufacturer & species & concentration \\
\hline AP-180 & SySy 155002 & rabbit & $1: 1000$ \\
\hline AP-2 & Abcam A2730 & mouse & $1: 1000$ \\
\hline ATP6V1A & Novus-Biologicals NBP1-33021 & rabbit & $1: 5000$ \\
\hline ATP6V1C1 & Abcam Ab87163 & rabbit & $1: 1000$ \\
\hline ATP6V1H & Abcam Ab187706 & rabbit & $1: 1000$ \\
\hline auxilin & babe (gift of Prof. De Camilli) & rabbit & $1: 1000$ \\
\hline bassoon & Enzo SAP7F407 & mouse & $1: 2000$ \\
\hline Chromogranin-A & Abcam ab45138 & rabbit & $1: 500$ \\
\hline Clathrin-heavy chain (HC) & Abcam AB2731 & mouse & $1: 500$ \\
\hline Clathrin-light chain (LC) & Abcam AB9884 & rabbit & $1: 2000$ \\
\hline dynamin (1, 2, 3) & BD Biosciences Cl 41 & mouse & $1: 2000$ \\
\hline EEA1 & ThermoFischer Scientific & rabbit & $1: 2000$ \\
\hline endophilin A1 & PA1-063A & goat & $1: 2000$ \\
\hline endophilin A2 & Santa Cruz L-18 & rabbit & $1: 1000$ \\
\hline endophilin A3 & Stimpy (gift of Prof.De Camilli) & rabbit & $1: 500$ \\
\hline GAPDH & Santa Cruz S-15 & rabbit & $1: 1000$ \\
\hline homer 1 & Sigma-Aldrich G9545 & rabbit & $1: 1000$ \\
\hline
\end{tabular}




\begin{tabular}{|l|l|l|l|}
\hline HPRT & Abcam 10479 & rabbit & $1: 2000$ \\
\hline intersectin-1 & gift of Prof.Haucke, Berlin & rabbit & $1: 1000$ \\
\hline intersectin-2 & Sigma-Aldrich HPA036475 & rabbit & $1: 1000$ \\
\hline Munc 13-2 & Gift of Prof. Brose & rabbit & $1: 1000$ \\
\hline Munc18-1 & SySy 116002 & rabbit & $1: 1000$ \\
\hline $\begin{array}{l}\text { Proton ATPase (Vo -116 kDa } \\
\text { subunit) }\end{array}$ & SySy 109003 & rabbit & $1: 1000$ \\
\hline rab27 & SySy 168003 & rabbit & $1: 1000$ \\
\hline rab3 & SySy Cl 42.1 & mouse & $1: 1000$ \\
\hline rabconnectin-3a & Sigma-Aldrich HPA039375 & rabbit & $1: 500$ \\
\hline rabconnectin-3b (WDR7) & Santa Cruz & rabbit & $1: 250$ \\
\hline rabphilin & SySy 118002 & rabbit & $1: 1000$ \\
\hline SNAP25 & SySy 116002 & rabbit & $1: 1000$ \\
\hline synapsin & SySy 106002 & rabbit & $1: 5000$ \\
\hline synaptobrevin-2 & SySy Cl 69.1 & mouse & $1: 2000$ \\
\hline synaptophysin & SySy Cl G96 & rabbit & $1: 1000$ \\
\hline synaptotagmin-1 & SySy 105011 & Mouse & $1: 2000$ \\
\hline synaptotagmin-7 & SySy 105173 & rabbit & $1: 1000$ \\
\hline syntaxin & SySy 1100111 & rabbit & $1: 1000$ \\
\hline VGAT & SySy Cl 117G4 & mouse & $1: 2000$ \\
\hline VGLUT1 & Abcam AB5905 & guinea & $1: 5000$ \\
& pig & \\
\hline
\end{tabular}

Table 8 - Secondary antibodies used for western blot analysis

\begin{tabular}{|l|l|l|l|l|}
\hline Target species & Tag & Manufacturer & Species & concentration \\
\hline Rabbit & HRP & Cell Signaling & Goat & $1: 4000$ \\
\hline Rabbit & IR 680 & Li-Cor & Goat & $1: 10000$ \\
\hline Rabbit & IR 800 & Li-Cor & Goat & $1: 10000$ \\
\hline Mouse & IR 680 & Li-Cor & Goat & $1: 10000$ \\
\hline Mouse & IR 800 & Li-Cor & Goat & $1: 10000$ \\
\hline Goat & IR 800 & Li-Cor & Donkey & $1: 10000$ \\
\hline Guinea pig & IR 800 & Li-Cor & Donkey & $1: 10000$ \\
\hline
\end{tabular}

\subsection{Plasmids}

The list of plasmids used in the study is in Table 9. Plasmids were used to express the mentioned protein in non-neuronal cells or neurons. 
Table 9 - Plasmids used in the study

\begin{tabular}{|c|c|c|c|}
\hline Plasmid name & Vector & Protein & $\begin{array}{l}\text { Fluorescence } \\
\text { marker }\end{array}$ \\
\hline $\mu 2$-EGFP & pCMV_EGFP-N1 & $\begin{array}{l}\mu 2 \text { subunit of AP2 } \\
\text { complex }\end{array}$ & EGFP \\
\hline BL150 & $\begin{array}{l}\text { iCre-NLS-RFP- } \\
\text { P2A }\end{array}$ & Cre & RFP \\
\hline CLC-EGFP & pCAG_EGFP & clathrin light chain & EGFP \\
\hline FUGW-rendoA1 & $\begin{array}{l}\text { FUGW-IRES- } \\
\text { EGFP }\end{array}$ & endophilin A1 & EGFP \\
\hline FUGW-rendoA1-BAR & $\begin{array}{l}\text { FUGW-IRES- } \\
\text { EGFP }\end{array}$ & $\begin{array}{l}\text { endophilin A1-BAR } \\
\text { domain }\end{array}$ & EGFP \\
\hline FUGW-rendoA1- $\Delta$ ITSN & $\begin{array}{l}\text { FUGW-IRES- } \\
\text { EGFP }\end{array}$ & \begin{tabular}{|l|} 
endophilin A1- \\
E329K+S366K
\end{tabular} & EGFP \\
\hline FUGW-rendoA2 & $\begin{array}{l}\text { FUGW-IRES- } \\
\text { EGFP }\end{array}$ & endophilin A2 & EGFP \\
\hline FUGW-rendoA2-BAR & $\begin{array}{l}\text { FUGW-IRES- } \\
\text { EGFP }\end{array}$ & $\begin{array}{l}\begin{array}{l}\text { endophilin A2-BAR } \\
\text { domain }\end{array} \\
\end{array}$ & EGFP \\
\hline mRFP-C-LC & mRFP-pcDNA3 & clathrin light chain & mRFP \\
\hline mRFP-EndoA1 & mRFP-pcDNA3 & endophilin A1 & mRFP \\
\hline mRFP- $\sigma 2$ & mRFP-pcDNA3 & $\begin{array}{l}\sigma 2 \text { subunit of AP2 } \\
\text { complex }\end{array}$ & mRFP \\
\hline Rbcn-3a GFP & pCMV_EGFP-N1 & rabconnectin-3a & EGFP \\
\hline Rbcn-3a-CAG-GFP & $\begin{array}{l}\text { pCAG_EGFP_N } \\
1\end{array}$ & rabconnectin-3a & EGFP \\
\hline V1A-EGFP & pCMV_EGFP-N1 & $\begin{array}{l}\text { V1A subunit of v- } \\
\text { ATPase }\end{array}$ & EGFP \\
\hline V1B2-td.tomato & Ptd.tomato-N1 & $\begin{array}{l}\text { V1B2 subunit of } v- \\
\text { ATPase }\end{array}$ & td.tomato \\
\hline V1H-EGFP & pAC_GFP1-C2 & $\begin{array}{l}\text { V1H subunit of } v- \\
\text { ATPase }\end{array}$ & EGFP \\
\hline VGLUT1-pHluorin & pCAG_N1 & $\begin{array}{l}\text { pHluorin tagged to } \\
\text { VGLUT1 lumenal } \\
\text { domain }\end{array}$ & pHluorin \\
\hline
\end{tabular}




\subsection{Primers}

Primers used for genotyping and quantitative PCR are listed below in Table 10 and 11.

Table 10 - Primers used for genotyping samples

\begin{tabular}{|l|l|}
\hline Primer & Sequence \\
\hline Dmxl2-3Arm & GGAGAGCGGACACTAGTCCCTTGG \\
\hline Dmxl2-5Arm & ATCTGTGCCACAGACCTAGGAAGC \\
\hline Dmxl2-LAR3 & CAACGGGTTCTTCGTTGTCC \\
\hline mEndo1-for & CCACGAACGAACGACTCCCAC \\
\hline mEndo1-RV1-WT & CGCACCTGCACGCGCCCTACC \\
\hline mEndo1-RV3-Neo & TCATAGCCGAATAGCCTCTCC \\
\hline mEndo2-Ko-For & CCTAGGGGCTTGGGTTGTGATGAGT \\
\hline mEndo2-Ko-Rev & CGTATGCAGCCGCCGCATTGCATC \\
\hline mEndo2-WT-For & CTTCCTGCCTTGCTGCCTTCCTTA \\
\hline mEndo2-WT-Rev & GCCCCACAACCTTCTCGCTGAC \\
\hline mEndo3_KO-R & CAACGGACAGACGAGAGATTC \\
\hline mEndo3_LOX-R & TGTGACAGTGGTGACCACAG \\
\hline mEndo3_SDL-F & CTCCCCATGGTGGAAAGGTCCATTC \\
\hline
\end{tabular}

Table 11 - Primers used for quantitative PCR

\begin{tabular}{|l|l|l|}
\hline Primer & Sequence (5'-3')-Forward & Sequence (5'-3')-Reverse \\
\hline endophilin A1 & GCAACTTTGGTCCTGCTCTC & GAATCTTGCCTTGTCGCTTC \\
\hline endophilin A2 & GAAGGGACCAAACTGGATGA & ATGCACTCTCCCAACAGA CC \\
\hline endophilin A3 & CACCTGAGAAAGCTGGAAGG & GCTCCTGGAGGATCTCTGTG \\
\hline Hprt & CCTCCTCAGACCGCTTTTT & AACCTGGTTCATCATCGCTAA \\
\hline
\end{tabular}




\subsection{Equipment}

All equipment used for this thesis is listed in Table 12.

Table 12 - List of equipments used in this study

\begin{tabular}{|l|l|}
\hline Equipment & Manufacturer \\
\hline Balance EW2200-2NM & Kern\&Sohn \\
\hline $37^{\circ}$ C Incubator & Memmert \\
\hline Accurpette & VWR \\
\hline Amaxa 4D-Nucleofector & Lonza \\
\hline Bio Photometer & Eppendorf \\
\hline Cell cracker & In-house workshop \\
\hline Dounce homogenizer & Wheaton \\
\hline EPC10 Amplifier & HEKA \\
\hline Examiner Z1 LSM710 & Zeiss \\
\hline microscope & \\
\hline Fine balance ALJ 120-4 & Kern\&Sohn \\
\hline Heating plate Mr3001 & Heidolph \\
\hline Hood Herasafe & Thermo Scientific \\
\hline Imaging chamber & home-made (workshop) \\
\hline Incubator Heracell 150i & Thermo Scientific \\
\hline Isolation cage & home-made (workshop) \\
\hline LSM 880 Airyscan microscope & Zeiss \\
\hline LSM800 microscope & Zeiss \\
\hline Mastercycler epgradient & Eppendorf \\
\hline Micromanipulator & home-made (workshop) \\
\hline NanoDrop Spectrophotometer & Peqlab \\
\hline Odyssey Infrared Imager & Li-Cor \\
\hline Patch pipette puller & Sutter instruments \\
\hline Perfusion head controller & ALA Scientific Instruments \\
\hline Pipettes & Eppendorf Research \\
\hline Platinum electrode stimulator & In-house built \\
\hline Power source (SDS-PAGE) & Bio-Rad \\
\hline Revolver wheel & Labnet \\
\hline S120AT2 ultracentrifuge rotor & Sorvall \\
\hline SevenEasy pH Meter & Mettler Toledo \\
\hline Table centrifuge (5424) & Eppendorf \\
\hline Thermomixer comfort & Eppendorf \\
\hline Ultra View Spinning Disk & Nikon/Perkin Elmer \\
\hline
\end{tabular}




\begin{tabular}{|l|l|}
\hline UV imaging system & Intas \\
\hline Western blot system & Bio-Rad \\
\hline Zeiss AxioObserver (TIRF) & Zeiss \\
\hline
\end{tabular}

\subsection{Software}

The software used in this study for data acquisition and analysis are listed in Table 13.

Table 13 - List of software programs

\begin{tabular}{|l|l|l|l|}
\hline Software & Company & City/State & Country \\
\hline Adobe Illustrator CS5.1 & Adobe Systems Inc. & San Jose, CA & USA \\
\hline Fiji & NIH & Bethesda, MD & USA \\
\hline Igor & Wavemetrics & Portland & USA \\
\hline Image Studio & Li-cor Bioscience & Bad Homburg & Germany \\
\hline Imaris 8.0.2 & Bitplane & Zurich & Switzerland \\
\hline MATLAB & Mathworks & Natick, MA & USA \\
\hline Microsoft Office & Microsoft Corporation & Redmond, WA & USA \\
\hline Mini Analysis 6.0.3 & Synaptosoft Inc. & New Jersey & USA \\
\hline Patch Master & HEKA electronics & Lambrecht/Pfalz & Germany \\
\hline Prism 6 & GraphPad & La Jolla, CA & USA \\
\hline Pulse/PULSEFIT & HEKA electronics & Lambrecht/Pfalz & Germany \\
\hline Volocity & PerkinElmer & Rodgau & Germany \\
\hline ZEN black & Carl Zeiss AG & Oberkochen & Germany \\
\hline ZEN blue & Carl Zeiss AG & Oberkochen & Germany \\
\hline
\end{tabular}




\section{Results}

\subsection{Endophilin-A controls recruitment, priming and fusion of neurosecretory vesicles}

Sindhuja Gowrisankaran ${ }^{1}$, Vicky Steubler ${ }^{1}$, Sébastien Houy², Johanna G. Peña del Castillo, Monika Gelker ${ }^{1}$, Jana Kroll', Paulo S. Pinheiro ${ }^{2}$, Nils Halbsgutt ${ }^{1}$, Nuno Raimundo ${ }^{3}$, Jakob B. Sørensen ${ }^{2}$, Ira Milosevic ${ }^{1, \#}$

${ }^{1}$ European Neuroscience Institute (ENI), Synaptic Vesicle Dynamics Group; University Medical Center Göttingen (UMG), Göttingen, Germany

${ }^{2}$ University of Copenhagen, Department for Neuroscience, Faculty of Health and Medical Science, Copenhagen, Denmark

${ }^{3}$ Institute for Cellular Biochemistry, University Medical Center Göttingen (UMG), Göttingen, Germany

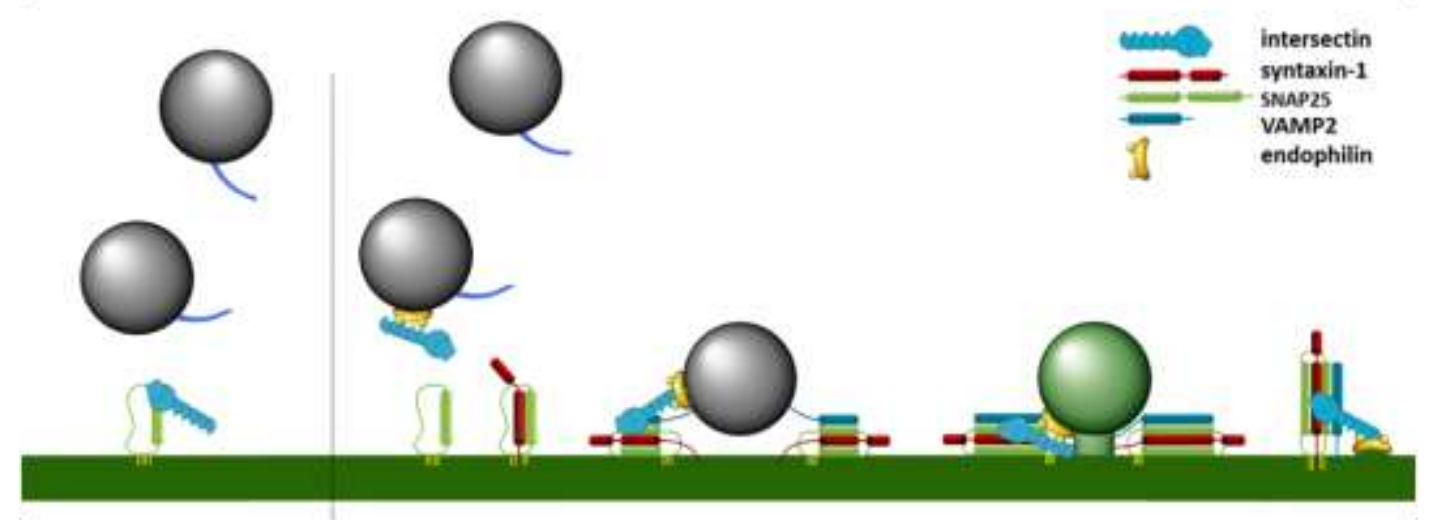

- Recruitment, priming and fusion of secretory vesicles is controlled by endophilin

- Lack of endophilins alters the distribution of secretory vesicles near the PM

- Endophilin's role in exocytosis is mediated through its SH3-domain

- Endophilin regulates intersectin localization by keeping it away from the PM 


\section{Endophilin-A controls recruitment, priming and fusion of neurosecretory vesicles}

Sindhuja Gowrisankaran', Vicky Steubler ${ }^{1}$, Sébastien Houy², Johanna G. Peña del Castillo, Monika Gelker ${ }^{1}$, Jana Kroll ${ }^{1}$, Paulo S. Pinheiro ${ }^{2}$, Nils Halbsgut ${ }^{1}$, Nuno Raimundo ${ }^{3}$, Jakob B. Sørensen², Ira Milosevic ${ }^{1, \#}$

${ }^{1}$ European Neuroscience Institute (ENI), Synaptic Vesicle Dynamics Group; University Medical Center Göttingen (UMG), Göttingen, Germany

${ }^{2}$ University of Copenhagen, Department for Neuroscience, Faculty of Health and Medical Science, Copenhagen, Denmark

${ }^{3}$ Institute for Cellular Biochemistry, University Medical Center Göttingen (UMG), Göttingen, Germany

"Corresponding author:

Dr Ira Milosevic

E-mail: imilose@gwdg.de

European Neuroscience Institute (ENI)

University Medical Center Göttingen (UMG)

Göttingen, Germany

\section{SUMMARY}

Endophilins-A are conserved endocytic adaptors with membrane curvature-sensing and -inducing properties. We show here that, independently of their role in endocytosis, endophilin-A1 and endophilin-A2 regulate exocytosis of neurosecretory vesicles. The number of neurosecretory vesicles was not altered in chromaffin cells without endophilin, yet fast capacitance and amperometry measurements revealed reduced exocytosis, smaller vesicle pools and changed fusion kinetics. Both endophilin-A1 (brain-enriched) and A2 (ubiquitous) rescued exocytic defects, but endophilin-A2 was more efficient. Distribution of neurosecretory vesicles was altered in the plasma membrane proximity, but levels and distributions of main exocytic and endocytic factors were unchanged, and slow compensatory endocytosis was not robustly affected. Endophilin's role in exocytosis is mediated through its $\mathrm{SH} 3$-domain and, at least in part, interaction with intersectin, a coordinator of exocytic and endocytic traffic. Altogether, we report that endophilinsA, key endocytic proteins linked to neurodegeneration, directly regulate exocytosis by controlling vesicle recruitment, priming and fusion. 


\section{INTRODUCTION}

Exocytosis-mediated release of vesicular content governs numerous biological events, including neurotransmission and neuromodulation, which are mandatory for brain function and survival. Following exocytosis, endocytosis rapidly retrieves the exocytosed vesicle membrane and proteins. The two processes are tightly coordinated, yet molecular mechanisms underlying such coupling are not well understood (Wu et al., 2014).

Endophilin-A (henceforth endophilin) is one of the best-characterized endocytic adaptors known to orchestrate various steps in clathrin-dependent and clathrin-independent endocytosis in mice, flies and nematodes (Ringstad et al, 1997; de Heuvel et al. 1997, Takei et al. 1999; Verstreken et al, 2002; Schuske et al, 2003; Milosevic et al., 2011; Boucrot et al., 2015; Renard et al, 2015). It contains an $\mathrm{N}$-terminal Bin-Amphiphysin-Rvs (BAR)-domain that senses and induces membrane curvature, and a C-terminal SH3-domain that mediates protein interactions (de Heuvel et al., 1997; Ringstad et al., 1997). Through those two domains endophilin acts as hub of a protein network that coordinates membrane remodeling, bud constriction and scission with cargo packing, actin assembly and recruitment of factors needed for fission and/or uncoating (as reviewed in Kjaerulff et al., 2011; Milosevic et al., 2018).

Unlike invertebrates that have only one endophilin gene, vertebrates express three endophilins encoded by three genes: SH3GL2 (endophilin 1, brain-enriched), SH3GL1 (endophilin 2, ubiquitous) and SH3GL3 (endophilin 3, brain and testis-enriched). Genetic and targeted ablation studies both at invertebrate and vertebrate synapses demonstrated that the lack of functional endophilin results in defective endocytosis and an accumulation of CCVs, revealing a phenotype that is most similar to the loss of the phosphatase synaptojanin-1 (Verstreken et al, 2002; Schuske et al, 2003; Milosevic et al., 2011). Besides synaptojanin-1, other prominent endophilin interactors include dynamins, the GTPases that mediate membrane fission (Ringstad et al., 1999). Endophilin was also proposed to have a central role in clathrin-independent endocytosis (Boucrot et al, 2015; Renard et al, 2015; Simunovic et al., 2017), and, most recently, in ultrafast endocytosis (Watanabe et al., 2018).

In addition to the well-established role in endocytosis, several indications point to endophilin's additional roles at the neuronal synapse. Specifically, endophilin interacts with synaptic vesicle (SV)-resident vesicular glutamate transporter 1 (vGLUT1; Vinatier et al., 2006; Voglmaier et al., 2006), and it was shown to undergo an association-dissociation cycle with SVs in the worm's 
nerve terminal as well as to be delivered to the periactive zone by exocytosis (Bai et al., 2010). Curiously, murine neurons missing all endophilins showed a reduced number of SVs and impaired neurotransmission (Milosevic et al., 2011). Yet, the synapse relies on local SV recycling where endocytosis is coordinated with exocytosis, so it is not clear whether the impaired neurotransmission results from defective endocytosis and SV recycling, or endophilin's role in exocytosis, or both.

Here, we show that endophilin, independently of its endocytic roles, is directly involved in the regulation of exocytosis. While exocytic and endocytic processes are tightly coupled at the synapse, in neurosecretory cells such coupling is less prominent given that the large dense-core vesicles (LDCVs) originate from the trans-Golgi network and undergo an hour(s) long maturation process before they fuse with the plasma membrane (Bader et al., 2002). Thus, we employed adrenal chromaffin cells (modified sympathetic neurons), a well-established model to study calcium-regulated exocytosis given that these cells use similar molecular machinery and release catecholamine at rates comparable to neurons in the central nervous system (Neher, 2006). After LDCVs fuse with the plasma membrane, an orchestrated process of compensatory endocytosis efficiently removes added membrane and proteins, and delivers them to a near-Golgi area, a process that takes tens of minutes (Houy et al., 2013). We found out that, like neurons, adrenal chromaffin cells contain all endophilins, thus, we employed murine chromaffin cells without endophilins (obtained from mice described in Milosevic et al., 2011) to decipher the role of endophilin in exocytosis.

\section{RESULTS}

\section{Endophilin-A is enriched at the neurosecretory vesicles in chromaffin cells}

Chromaffin cells contain numerous LDCVs that release their content into the blood by fast exocytosis (Neher, 2006). To check if endophilin is expressed in adrenal chromaffin cells, we looked for the presence of three endophilins' mRNAs and proteins. Firstly, RNA was isolated from cells extracted from the center of adrenal gland (medulla) obtained from wild-type (WT) and endophilin 1, 2, 3 triple knock-out (TKO) PO mice. All three endophilin mRNAs were detected by real-time quantitative PCR (qPCR) in the WT (endophilin 2 signal was the most prominent), but not in the TKO samples (Figure 1A). Next, Western blots revealed the presence of endophilin 1 
and endophilin 2 in the adrenal gland homogenate from WT P0 mice, whereas these proteins were absent in the glands obtained from TKO mice (Figure 1B). Endophilin 3, known to be the least abundant member of the endophilin family in the brain (Milosevic et al., 2011), could not be detected in $50 \mu \mathrm{g}$ of adrenal gland homogenate by Western blotting (Figure 1B), suggesting that the very small amounts of endophilin 3 mRNA detected in chromaffin cells are not efficiently translated in this system.

The studies of native endophilins' distribution in mammalian cells are hindered by the lack of specific antibodies for immunostainings. Based on the overexpression studies, endophilins are primarily cytosolic proteins that associate with membranes in various cells (Ringstad et al., 2001; Perera et al., 2006; Milosevic et al, 2011; Murdoch et al, 2016). To check for the distribution of endophilins in chromaffin cells, we generated two new anti-endophilin antibodies and tested their specificity on knock-out cells and tissues. These antibodies, termed endo-A1gp and endo-A2rab respectively, gave almost no staining in endophilin TKO cells (Figure 1C and 1D, right panels) and revealed that the majority of endophilin 1 and 2 was cytosolic (Figure 1C and 1D, left panels). Yet, a part of endophilin 1 and endophilin 2-specific signal was punctate and reminiscent of signal obtained with the LDCV makers, e.g. chromogranin-A (CgA) (Figure 1C and 1D). When chromaffin cells were co-immunostained for $\mathrm{CgA}$ and endophilin 1 (or endophilin 2, respectively), a significant colocalization between $\mathrm{CgA}$ and endophilins was detected (Figure 1E and 1F; note that the colocalization values were corrected for an accidental colocalization given the high abundance of both signals).

To test if endophilin can indeed associate with the secretory vesicles, we purified LDCVs from the medulla of bovine adrenal glands by adapting the published protocol (Suppl Fig S1A; Park et al., 2012; mouse adrenals glands are small, 1-2 mm in size, and they did not provide sufficient amount of starting material), and we isolated SVs from the mouse brains (Farsi et al., 2018). Immunoblotting revealed that endophilin 1 was present in the purified LDCV as well as in the purified SV samples (Suppl Fig S1B; note that endophilin 1 was detected in adrenal gland homogenate in Figure 1B, but not here since the lower sample amounts were loaded to avoid Western blot signal saturation).

Having specific antibodies against endophilins 1 and 2 for immunostaining now available, we inspected the distribution of endophilin 1 and 2 in chromaffin cells upon stimulation (by $59 \mathrm{mM}$ potassium solution). Interestingly, a mild enrichment of endophilin 1 and endophilin 2-specific 
signals was detected near/at the plasma membrane upon stimulation (Suppl Fig S1C-D, graphs show the line intensity profiles through chromaffin cells).

In sum, a fraction of endophilin-specific signal in chromaffin cells colocalized with LDCVs and translocated to the plasma membrane upon stimulation. LDCVs are derived from Golgi and undergo a long maturation process before their release, thus the presence of endophilin on LDCVs is unexpected, in particular in the light of two decades long research on this protein family. The function(s) of endophilin associated with the neurosecretory vesicles and in exocytosis are entirely unknown.

\section{Endophilin-A promotes exocytosis in chromaffin cells}

To check whether endophilins have a role in exocytosis, we performed fast electrophysiological and electrochemical measurements that combined membrane capacitance and amperometry recordings on chromaffin cells of endophilin TKO mice and corresponding littermate control (endophilin $\mathrm{A}^{-{ }^{-}-} \mathrm{A} 2^{+/+}-\mathrm{A} 3^{-/-}$, henceforth endophilin KOWTKO or $\mathrm{KWK}$; note that a direct comparison to wild-type, WTWTWT, littermates was not possible since the strain was knockout for endophilin A1 and A3). The chromaffin cells were isolated from adrenal glands at P0 (for experimentation, the cells were used between 2-4 days-in-vitro, DIV). Fast capacitance and amperometry measurements were performed as follows: each cell was loaded (via a patch pipette) with the photo-labile $\mathrm{Ca}^{2+}$-chelator nitrophenyl-EGTA and two $\mathrm{Ca}^{2+}$-dyes (Fura-4F and Furaptra) to enable accurate measurements of intracellular calcium concentrations $\left(\left[\mathrm{Ca}^{2+}\right]_{i}\right)$ during the whole experiment. Photolysis of caged $\mathrm{Ca}^{2+}$ compound increased $\left[\mathrm{Ca}^{2+}\right]_{\mathrm{i}}$ from several hundred $\mathrm{nM}$ to above $10 \mu \mathrm{M}$, resulting in a robust exocytosis, which was assayed by an increase in membrane capacitance and amperometric current, one cell at a time. The measured increase in membrane capacitance is proportional to the change in chromaffin cell plasma membrane surface area, while simultaneous amperometric recordings allowed the measurements of secreted catecholamine during the exocytic events (Milosevic et al., 2005; Nagy et al., 2005; Neher 2006). Remarkably, both capacitance and amperometry measurements from endophilin TKO chromaffin cells revealed reduced exocytosis $(340 \pm 36 \mathrm{fF})$ when compared to the control littermate $(501 \pm 30$ fF for KOWTKO) after the first stimulation (Figure 2A-B). We noted that exocytosis from endophilin KOWTKO cells was comparable to the exocytosis from wild-type cells originating from several different C57/BL6-based strains that have been recorded over the years in different 
laboratories (e.g. Sørensen et al., 2003; Borisovska et al., 2005; Liu et al., 2008; Schonn et al., 2008; Pinheiro et al., 2014; Kedar et al., 2015; Man et al., 2015). Analysis of capacitance measurements revealed that the exocytic burst $(189 \pm 25 \mathrm{fF}$ in TKO vs. $299 \pm 22 \mathrm{fF}$ in KOWTKO, Figure 2C) and the sustained component (151 $\pm 16 \mathrm{fF}$ TKO vs. $202 \pm 14 \mathrm{fF}$ in KOWTKO; Figure 2D) were reduced. Further analysis of the exocytic burst revealed that both readily releasable pool (RRP) and slowly releasable pool (SRP) sizes (the amplitudes of the exponential fits) were significantly smaller ( $78 \pm 15 \mathrm{fF}$ vs. $142 \pm 13 \mathrm{fF}$ and $54 \pm 10 \mathrm{fF}$ vs. $91 \pm 11 \mathrm{fF}$, respectively; Figure 2E). The fusion kinetics of the RRP vesicles from the TKO cells (time constants of the exponential fits) was sped up (9.0 $\pm 1.0 \mathrm{~ms}$ vs. $12.1 \pm 1.0 \mathrm{~ms}$; Figure 2F). Curiously, we found that the RRP time constant correlated with the RRP size (Suppl Fig S2; also noted in synaptotagmin-7 KO, Schonn et al., 2008), suggesting that the faster fusion kinetics and lower amplitude may not be two phenotypes, but one. The kinetic parameter of the SRP was not significantly altered, although on average it was slower in the TKOs (Figure 2G). Altogether, these data indicate that endophilin controls the size of releasable pools and the rate of RRP vesicle fusion. Similar results were found upon a second stimulation applied 100s after a first stimulation (Figure 2H-K; small exocytic responses prevented reliable exponential fitting so the vesicle pool and kinetic analyses could not be performed). Interestingly, the ratio between second and first burst was not changed (Figure 2L), suggesting that vesicle pools could be efficiently refilled between two stimuli.

To verify that the effect of lack of endophilin on exocytosis is specific, and to test for the contribution of individual endophilins to the chromaffin cell secretion, we expressed full length endophilin 1, or endophilin 2 respectively, in endophilin TKO cells using a bicistronic lentiviral system, and performed electrophysiological measurements as before. The lentiviral expression system was verified in the HEK-293 cells (Suppl Fig S3A). The co-expression of enhanced green fluorescent protein (EGFP) through the IRES system was used as a marker of expression in each recorded cell, and it indicates that proteins were expressed to similar levels (Suppl Fig S3B). Remarkably, both endophilin 1 and endophilin 2 were able to rescue exocytosis in TKO cells (Figure 3A-B; the rescue with endophilin 2 was indistinguishable from endophilin KOWTKO cells). Both burst and sustained exocytic components were efficiently rescued by either endophilins (burst: $221 \pm 28 \mathrm{fF}$ for endophilin 1 and $292 \pm 43 \mathrm{fF}$ for endophilin 2 vs. $137 \pm 15 \mathrm{fF}$ measured in endophilin TKO; sustained: $190 \pm 23 \mathrm{fF}$ for endophilin 1 and $178 \pm 20 \mathrm{fF}$ for endophilin 2 vs. $92 \pm 24 \mathrm{fF}$ in endophilin TKO; Figure $3 \mathrm{C}$-D), as well as the RRP (Figure $3 \mathrm{E}$ ) and the fusion 
kinetics of the RRP (Figure 3F). Although the kinetics of SRP was faster when either endophilin 1 or 2 were expressed, the difference was not significant (Figure 3G). Similar results were noted upon a second stimulation (Suppl Fig S3C-F). Notably, the releasable pools were recovered efficiently between two stimuli that were 100s apart, as revealed by an unchanged ratio between second and first burst (Suppl Fig S3G). Altogether, these data reveal that the effect of endophilin on exocytosis was specific, and that the expression of either endophilin 1 or 2 was sufficient to support exocytosis in chromaffin cells.

To inspect whether chromaffin cells without endophilin show qualitative changes in vesicle fusion, we examined the properties of single amperometric events from endophilin TKO cells and TKO cells expressing endophilin 2 . Here, secretion was elicited by loading chromaffin cells in a wholecell mode with low calcium intrapipette solution, as detailed in Methods. Representative amperometric traces for endophilin TKO, and endophilin TKO expressing endophilin 2 are shown in Figure 3H; the analyzed properties of the single spike are illustrated in Figure 31. Significant differences were observed in the number of spikes/cell (Figure 3J) and in the single spike charge and amplitude (Figure 3K-L) - all these parameters were found to be decreased in endophilin TKO cells, while the foot properties and single spike rise time were not changed (Figure $\mathbf{3 M - Q}$ ). Given that exocytosis in chromaffin cells happens on the millisecond-to-second time scale, these data strongly suggest that endophilin has a direct role in exocytosis by controlling the sizes and fusion of releasable vesicle pools.

\section{LDCV vesicle morphology and number are not altered in the absence of endophilin-A}

The decreased exocytosis in endophilin TKO chromaffin cells suggest that these cells might have less LDCV able to undergo exocytosis, or that the exocytic process itself is affected, or both. To distinguish between these possibilities, we performed comprehensive ultrastructural studies by combination of electron microscopy (EM) and 3D-confocal imaging.

Ultrastructural EM analyses on chromaffin cells in the adrenal gland were made as detailed in Methods. We noted that the overall size and morphology of endophilin TKO chromaffin cells was unaltered in comparison to controls (WT and littermate endophilin KOWTKO cells) (Figure 4A), as well as the LDCV size (Figure 4D). Curiously, the average number of LDCVs per cell (Figure 4B), or per cell area (Figure 4C), were not significantly different between endophilin TKO, control 
littermates and WTs. This observation rules out the possibility that endophilin affects exocytosis by altering the number of LDCVs in the cell. Yet, while studying the distribution of LDCVs in endophilin TKO cells, we noticed that LDCVs were distributed further away from the plasma membrane in comparison to the controls (Figure 4A bottom panels, Figure 4E-F'), and less LDCVs were found within $10 \mathrm{~nm}$ from the plasma membrane (Figure 4F), suggesting a problem with vesicle recruitments to the release sites.

The ultrastructural analysis was complemented by two independent approaches using confocal microscopy and Western blotting. First, fixed WT, endophilin KOWTKO and TKO chromaffin cells were immunostained against cargo marker $\mathrm{CgA}$, and the whole cell (3D image acquired thorough z-stacks) was imaged by the Zeiss Airyscan system. Representative images are shown in Figure 5A. Quantification of LDCV number in the whole cell volume revealed similar number of LDCVs in endophilin TKO and controls (Figure 5B). Concordantly, the levels of $\mathrm{CgA}$ protein were not altered in endophilin TKO adrenal gland homogenates (Suppl Fig S4A-B).

Altogether, chromaffin cells without endophilin displayed minor alterations in the LDCV distribution, but the reduced exocytosis in these cells was not a result of the altered vesicle number, morphology or cargo (i.e. CgA) amount.

\section{Protein machinery involved in the exocytic process is not altered in chromaffin cells without endophilin-A}

The reduced exocytosis (observed by fast electrophysiological measurements) in endophilin TKO cells occurs at the milliseconds-to-seconds time scale. Endophilins, being major endocytic proteins, could have an indirect effect on the LDCV composition and/or membrane and protein recycling processes in chromaffin cells. Despite the overall abundance of LDCVs and the unaltered morphology of individual LDCVs in endophilin TKO chromaffin cells, it is possible that some LDCVs may not be able to undergo exocytosis due to changes in accessory factors required for exocytosis. Specifically, SNAREs, Sec1/Munc-18 (SM)-proteins, Munc13s and other exocytic proteins may not be efficiently recycled from the previous rounds of exocytosis, or properly displayed on LDCVs in the absence of endophilin. Therefore, we checked the abundance and distribution of key exocytic proteins in endophilin TKO cells by immunocytochemistry (ICC) and Western blotting. 
As an exemplary exocytic protein, we first analyzed the abundance and distribution of synaptotagmin-1 (Syt-1), a Ca ${ }^{2+}$-sensor important for LDCV exocytosis in chromaffin cells. We immunostained endophilin TKO, endophilin KOWTKO and WT cells for Syt-1, and noticed that neither distribution (Figure 5C-D), nor protein levels Suppl Fig S4A-B), of Syt-1 were altered in endophilin TKO cells. We next checked whether proper amounts of Syt-1 are present on LDCVs by quantifying the intensity of Syt-1 signal on the $\mathrm{CgA}$-positive structures. The analysis showed no statistical difference of Syt-1 intensity on CgA-positive LDCVs between WT and endophilin TKO cells (Figure 5E).

We further inspected protein levels of Syt-1, as well as synaptophysin, several SNAREs (SNAP25, syntaxin-1, synaptobrevin-2/VAMP2) and Munc-18-1 in adrenal gland homogenates by Western blotting, and found no significant difference between endophilin TKO cells and controls (littermate endophilin KOWTKO and WT samples were used as controls; Suppl Fig S4 A-D). Next, the ICC for a number of exocytic proteins, namely SNAP-25, synaptobrevin-2/VAMP-2, Munc18-1 and synaptotagmin-7 revealed no significant changes in the protein level and distribution in endophilin TKO chromaffin cells (Figure 5F-G). Taken together with the unchanged number of LDCVs, these data suggest that the reduced catecholamine release in the absence of endophilin is likely a consequence of endophilin's direct action in exocytosis.

\section{Endocytic defects in the absence of endophilin-A in chromaffin cells}

Endophilin TKO cells show an unaltered number of LDCVs and normal distribution and abundance of main exocytic proteins, yet, it is possible that altered endocytosis affects exocytosis in chromaffin cells. While exocytosis is well studied in this model system, endocytic modes are far from understood. Two temporally and mechanistically distinct forms of endocytosis have been reported: rapid endocytosis that depends on dynamin-1 and GTP, and slow endocytosis that involves dynamin-2 and clathrin (Smith and Neher, 1997; Elhamdani et al., 2006). We predominantly studied slow (likely clathrin-mediated) endocytosis since it is not known to which extent these cells undergo fast local recycling.

First, we examined the protein levels and distributions of main endocytic factors, namely clathrin heavy chain (HC), adaptor protein 2 (AP2), adaptor protein 180 (AP180), dynamins 1, 2 and 3 by ICC and Western blotting. Except for clathrin HC whose levels were mildly elevated in the absence of endophilin (by ICC only, Figure 6A), we detected no difference in the overall levels of 
aforementioned proteins, both by Western blotting of adrenal gland homogenates (Figure 6B) or by quantifying immunofluorescence in chromaffin cells (Figure 6A). In addition, the distribution of AP2 and dynamin seemed unaltered in endophilin TKO cells (Figure 6A).

The slow endocytic recycling process in chromaffin cells was tested by three approaches: the uptake of transferrin-Alexa Fluor ${ }^{\mathrm{TM}} 546$ (A546; clathrin-dependent) and uptake of mCling-Atto647 or recombinant cholera toxin subunit B (CT-B)-Alexa Fluor 594 (A594; clathrin-dependent and independent). First, we looked at the 10 min-uptake of transferrin-A546 by analyzing $3 \mathrm{D}$ images (through z-stacks) of whole cell volume of endophilin TKO, endophilin KOWTKO and WT cells. The results were peculiar: while no significant difference between WT and endophilin TKO cells was observed (Figure 6C-D), endophilin TKO showed reduced amount of internalized transferrinA594 when compared to endophilin KOWTKO cells (Figure 6C-D).

We further inspected the uptake of the mCling-Atto647 that binds to the plasma membrane and whose internalization can be readily monitored for minutes (Revelo et al., 2016; Suppl Fig S5A). We first characterized the specificity of the mCling uptake in chromaffin cells by stimulating cells with high potassium in the presence of Pitstop-2 (clathrin coat formation inhibitor) and found that this inhibitor blocked the uptake of mCling efficiently (Suppl Fig S5B). When mCling-Atto594 was applied to chromaffin cells without endophilin, the number of internalized vesicles (mCling-positive structures) was comparable to controls (WT and endophilin KOWTKO cells; Figure 6E). Although a delay in kinetics in the first few minutes is a possibility in the TKO cells, the similar number of internalized vesicles was detected at 8 minutes (Figure 6F). A similar result was obtained with the uptake of CT-B-A594 (Suppl Fig S5C-D). Altogether, these data indicate that the lack of endophilin affects endocytosis in chromaffin cells only modestly and hence cannot account for the observed effect on exocytosis in this model system.

\section{Endophilin's BAR-domain is not sufficient to mediate exocytic release from chromaffin cells}

To get mechanistic insights on how endophilin regulates the exocytic process, we looked at the function of endophilin domains. Endophilins have two domains separated by a linker region: an $\mathrm{N}$-terminal BAR-domain that senses and introduces membrane curvature and a C-terminal SH3domain that mediates protein-protein interactions (e.g. with dynamin, synaptojanin-1, etc.). In nematodes, it has been shown that the endophilin's BAR domain is necessary and sufficient to 
mediate the role of this protein in endocytosis (Bai et al., 2010), while in mammalian cells both domains were needed (Milosevic et al., 2011). We first tested whether endophilin's BAR-domain alone (i.e. endophilin without SH3-domain) is sufficient to support exocytosis in chromaffin cells. We expressed endophilin 1 BAR-domain and endophilin 2 BAR-domain respectively (together with EGFP through the bicistronic IRES-expression system - the expression levels of all tested proteins were comparable; Figure 7I) in endophilin TKO cells, and performed capacitance and amperometry measurements as before. Interestingly, expression of endophilin 1 BAR-domain, as well as endophilin 2 BAR-domain, did not result in a rescue but rather in a further reduction of secretion from endophilin TKO cells, either during the first (Figure 7A-D) or the second stimulus (Figure 7E-H). The small responses revealed overall decrease in exocytosis, including both burst and the sustained component (first stimulus: Figure 7B-D; second stimulus Figure 7F-H). This dominant-negative effect reveals that the SH3-domain-mediated function is important for endophilin's role in exocytosis, and that the full-length protein is needed to support exocytosis in chromaffin cells.

\section{Endophilin's role in exocytosis is mediated, at least in part, through its interaction with intersectin}

Endophilin's SH3-domain is known to mediate its interaction with several proteins, yet only two of them have been implicated in chromaffin cell exocytosis: dynamins (González-Jamett et al., 2010; Chan et al., 2010; Anantharam et al., 2011) and intersectins (Malacombe et al., 2006; Yu et al., 2009; Momboisse et al., 2010; Gerth et al., 2017). Distribution and levels of dynamins were not altered (Figure 6A-B), as detailed above. Curiously, while levels of intersectin-1, a membraneassociated protein that coordinates exocytic and endocytic vesicle traffic, were comparable (Suppl Fig S6A-B; both short and long isoform of intersecin-1 are shown), the distribution of intersectin-1 was altered in chromaffin cells lacking endophilins (Figure 8A; the line-intensity profile through the depicted cells is shown below the images). A detailed examination in resting chromaffin cells revealed that the fraction of intersectin-1 on the plasma membrane was higher in the TKO compared to the WT (Figure 8B; Suppl Fig S6C-D). Upon stimulation, more intersectin1 was recruited to the plasma membrane in WT cells, whereas this redistribution did not happen in endophilin TKO cells (Figure 8B; Suppl Fig S6C-D). Similar observations were made with 
intersectin-2 (Figure 8C-D; Suppl Fig S6E-F). In sum, intersectin 1 and 2 are recruited to the plasma membrane (1) during stimulation and (2) in absence of endophilins.

To check if this effect is specific, we attempted to rescue the intersectin-1 distribution by expressing either endophilin 1 or endophilin 1-E329K+S366K (mutant that does not bind intersectin; Pechstein et al., 2015) in endophilin TKO cells. Upon endophilin 1 expression in TKO cells, the distribution of intersectin-1 resembled the protein distribution in WT cells (as detected by immunostaining - Figure 8E, compare to Figure 8A, quantification in Figure 8F). Remarkably, the expression of mutant endophilin 1-E329K+S366K did not have the same effect, and intersectin-1 was still mislocalized (Figure 8E-F). These data suggest that endophilin-intersectin interaction is important for intersectin distribution in chromaffin cells and that it regulates intersectin's access to the plasma membrane where the vesicle fusion happens.

Given that expression of endophilin 1 in endophilin TKO cells rescued exocytosis when inspected by combined fast capacitance and amperometry measurements (Figure 3A-G), we tested whether the same effect could be achieved by expressing the endophilin mutant that does not bind intersectin-1 (E329K+S366K, indicated as endoA1- $I T S N)$. The expression of endoA1$\triangle$ ITSN through bicistronic lentiviral system was tested first (Suppl Fig S6H). Remarkably, endophilin 1- $\triangle$ ITSN was not able to fully rescue exocytosis (Figure 8G-I), including the size and the time constants of vesicle pools (Figure $\mathbf{8 K}-\mathbf{N}$, note that Figure $\mathbf{8 J}$ depicts the sustained component that was at least partially rescued by endophilin 1- $\triangle$ ITSN). Thus, endophilin's role in exocytosis is mediated, at least in part, through its interaction with intersectin-1.

\section{DISCUSSION}

The first reports on endophilin linked its function to endocytosis (Ringstad et al., 1997; de Heuvel et al., 1997; Ringstad et al., 1999; Verstreken et al, 2002; Schuske et al, 2003). Over three hundred papers in the past 20 years built on these initial findings and helped to unveil the mechanisms of endophilin action in several types of endocytosis, including clathrin-dependent (Ringstad et al., 1999; Verstreken et al, 2002; Schuske et al, 2003; Milosevic et al., 2011), clathrinindependent (Boucrot et al, 2015; Renard et al, 2015; Simunovic et al., 2017; Boucrot et al, 2018) and ultrafast endocytosis (Watanabe at al., 2018).

Our study shows that, in addition to its role in endocytosis, endophilin also plays a key role in the recruitment, priming and fusion of neurosecretory vesicles (endophilin 1 and 2 have overlapping 
functions in exocytosis). Endophilin is a peripheral protein with membrane-binding properties that appears to be present on at least some secretory vesicles: we detected its presence on SVs and LDCVs as well as a significant colocalization between LDCV marker CgA and endophilin 1 and endophilin 2, respectively. Upon stimulation, a fraction of endophilin translocates to the plasma membrane. Endophilin's role in exocytosis is found to be mediated through its SH3-domain, most likely through its $\mathrm{SH} 3$-domain interactor intersectin, another membrane-associated protein that coordinates exocytosis and endocytosis and also translocates to the plasma membrane upon stimulation (see below). In the absence of endophilin, a significant fraction of intersectin is mislocalized to the plasma membrane, suggesting that endophilin acts as a repressor of intersectin by keeping intersectin away from the plasma membrane.

\section{Endophilin's role in exocytosis is direct and endocytosis-independent}

We consider that endophilin's role in exocytosis is direct: The expression of either endophilin 1 or endophilin 2 alone was sufficient to rescue all exocytic defects seen in neurosecretory cells without endophilin. These data also show that two endophilins have a redundant role in exocytosis. In addition, the expression of endophilin 1 BAR domain, or endophilin 2 BAR domain, was not sufficient to produce a rescue of exocytosis in endophilin TKO cells. Next, endophilin that cannot bind intersectin-1 (E329K+S366K; Pechstein et al., 2015) was not able to fully rescue exocytosis. Lastly, none of the main exocytic proteins tested was found to be altered in the cells without endophilin.

The newly discovered role of endophilin in exocytosis appears to be independent of its wellestablished functions in endocytosis: the recycling/uptake of proteins (e.g. transferrin, cholera toxin subunit-B uptake) and membrane (fluorescently-labelled mCling reported in Revelo et al. (2016) was characterized through the Pitstop-2 application and stimulations, and found to be specific) were not majorly altered. We observed a mild decrease in the transferrin uptake efficiency between endophilin TKO and endophilin KOWTKO cells, yet no difference was detected between endophilin TKO and WT. This is a peculiar finding since it suggests that endophilin KOWTKO cells were more efficient in the transferrin uptake than WTs. Although mCLING uptake by TKO chromaffin cells was not found to be significantly altered at 8 minutes, a delay in the uptake in the first few minutes is possible and so the release site-clearance could be affected (Hua et al., 2013). Given that the LDCV generation/maturation steps take tens of minutes to hours, 
the short initial endocytic delays are likely not relevant for the recycling of proteins and generation of new LDCVs.

Despite the key exocytic (SNAREs, synaptotagmins and Muncs) and endocytic (AP2, AP180 and dynamin) machineries were not altered in endophilin TKO chromaffin cells, it was necessary to check whether all LDCVs were fusogenic (i.e. whether SNAREs and other exocytic proteins were efficiently recycled from previous rounds of exocytosis, and whether they were properly localized at the LDCVs). A systematic evaluation of each $\mathrm{CgA}$-positive vesicle for synaptotagmin-1 presence and intensity revealed no major changes in the endophilin TKO chromaffin cells compared to the controls. In addition, we did not observe any overall differences in the levels and distributions of several additional vesicular proteins (e.g. VAMP2/synaptobrevin-2, synaptophysin, etc.).

\section{Endophilin's role in exocytosis is mediated through its SH3-domain and intersectin interaction}

We followed several leads that could explain endophilin's role in exocytosis. The absence of endophilin's SH3-domain reduced exocytosis even further than seen in endophilin TKO chromaffin cells, revealing a dominant negative effect of the BAR domain alone. Interestingly, out of all exocytic and endocytic (except clathrin) proteins inspected, only the distributions of intersectin-1 and intersectin-2 were altered. We reported previously that endophilin-intersectin-1 interaction is mediated through the SH3-domains of both proteins (Pechstein et al, 2015). We now found that an endophilin mutant that cannot bind intersectin-1 (E329K+S366K) was also not able to rescue the altered intersectin-1 distribution nor exocytic phenotype in endophilin TKO chromaffin cells. Both intersectin-1 and intersectin- 2 were initially discovered as scaffold proteins involved in endocytosis (Yamabhai et al., 1998; Hussain et al., 1999; Simpson et al., 1999; Koh et al., 2004; Marie et al., 2004; Pechstein et al., 2010; Sakaba et al, 2013). Yet, further investigations showed that intersectins are also implicated in several other processes, including exocytosis (Malacombe et al., 2006; Yu et al., 2009; Momboisse et al., 2010; Sakaba et al, 2013; Gerth et al., 2017), thereby suggesting a role for intersectins in the coupling of exocytosis and endocytosis (Okamoto et al., 1999; Gubar et al., 2013; Gerth et al., 2017). Curiously, we observed that a fraction of intersectin-1 and intersectin-2 could be found on purified LDCVs and SVs (Suppl 
Fig S6G). Taken together with previous reports, our data suggest that endophilin regulates vesicle recruitment and exocytosis through its interaction with intersectin.

Both EM and immunofluorescent analyses of $\mathrm{CgA}$-stained vesicles showed no changes in the number of secretory vesicles, thus, the reduction in exocytosis was not due to a reduced number of vesicles. However, curiously, the lack of endophilins had also altered the distribution of LDCVs near the plasma membrane. It is well known that the neuroendocrine cells (e.g. chromaffin cells) have a cortical F-actin barrier that controls the access of secretory vesicles to the plasma membrane (Trifaró et al., 1992). The polymerisation of the actin barrier seems primarily RhoAdependent, while the de novo synthesis of actin is Cdc42-dependent (Malacombe et al., 2006; Momboisse et al., 2009). In addition, oligophrenin (a key RhoA inactivator) was shown to bind endophilin and assists its role in endocytosis (Nakano-Kobayashi et al., 2009; Nadif Kasri et al., 2011). We observed that, in the absence of endophilins, actin barrier looks depolymerized in the resting condition, while stimulation increased actin's density (Suppl Figure S7) Thus, lack of endophilins led to a modulation of the actin barrier and the de novo synthesis. Given that actin is known to play a role in site clearance for vesicle docking and fusion (Miki et al., 2016), this perturbed actin dynamics may affect the availability of vesicles and their access to the plasma membrane, which in turn can explain the reduced exocytosis in the absence of endophilins. Therefore, the endophilin-intersectin interaction could also be a mechanism to control the actin barrier in chromaffin cells and subsequently the availability of vesicles for fusion. Once the actin barrier is depolymerized at the specific sites, secretory vesicles can be recruited to their site of release at the plasma membrane, possibly through an interaction between intersectin and the SNARE-protein SNAP-25 or SNAP-23 (Okamoto et al., 1999). Strikingly, chromaffin cells without intersectin-1 also showed reduced exocytosis (Yu et al., 2009), and intersectin was shown to regulate the replenishment of the fast-releasing synaptic vesicle pool in the calyx of Held synapse (Sakaba et al, 2013). We found that, without endophilin, intersectin mislocalized to the plasma membrane, LDCVs distribution was altered and exocytosis was diminished. In this context, endophilin may also be viewed as a regulator of intersectin in exocytosis.

\section{A putative model of endophilin's role in exocytosis}

This study, along with our previous results on endophilin and intersectin (Milosevic et al., 2011; Pechstein et al, 2015) and the reported interaction between intersectin and SNAP-25 and SNAP- 
23 (the SNARE proteins; Okamoto et al., 1999), as well as with the numerous intersectin studies (see above), suggests the following sequence of events (Suppl Fig S8). Endophilin is present at least on some neurosecretory vesicles and able to recruit intersectin. Endophilin and intersectin then act in tandem to stimulate the recruitment of those vesicles to the plasma membrane and their site of release, likely through intersectin's role in the actin network modulation and its interaction with the SNARE proteins at the plasma membrane. Such recruitment may also be supported by the property of endophilins to bind and stabilize curved vesicular membrane via their BAR domain.

When the neurosecretory vesicle reaches the plasma membrane, the SNARE complex can be built between the vesicular VAMP2/synaptobrevin-2 and plasma membrane-resident SNAP25 and syntaxin-1, and endophilin and intersectin may stabilize this process. Once recruited to the proximity of the plasma membrane, we propose that endophilin and intersectin do not dissociate from the plasma membrane but take part in the endocytic process that follows the fusion of secretory vesicles. As such, endophilin and intersectin may act as a scaffold that couples vesicle fusion (exocytosis) and fission (endocytosis) events. This premise is supported by intersectin's direct interaction with dynamin (Okamoto et al., 1999) and two studies in invertebrates: Bai et al. (2010) suggested that endophilin is delivered to endocytic zones by exocytosis in C. elegans, and Winther et al. (2013) showed that $D$. melanogaster's Dap160/intersectin mutants lacking dynaminbinding do not properly accumulate dynamin in the periactive zone. Importantly, we show that intersectin mislocalizes to the chromaffin cell plasma membrane without endophilin. Since endophilin can regulate intersectin localization, it may be part of a check-point mechanism to ensure that intersectin acts only at the optimal time. This model is created primarily based on data obtained from neuroendocrine cells: it is possible that endophilin (and intersectin)'s roles in exocytosis diverge at the neuronal synapses where actin's role may differ. Further studies are needed to address this question.

In conclusion, our study suggests that endocytosis of neurosecretory vesicles is dependent on endophilin 1 and endophilin 2, that were so far considered only as endocytic adaptors that form and stabilize membrane curvature. This novel function of endophilin is dependent on intersectin, and most likely actin. 


\section{ACKNOWLEDGEMENTS}

We thank the UMG animal facility, D. Schwitters, M. König and M. Costa for the excellent assistance and for help with genotyping, P. De Camilli (Yale University) and V. Haucke (FMP) for reagents and discussion, and $A$. Milosevic for drawing the model. This work was supported by Schram-Stiftung T287/25457 and Deutsche Forschungsgemeinschaft (Emmy Noether Young Investigator Award MI-1702/1) to IM, SySy fellowship to SG, the Lundbeck foundation (PSP, SH, JBS), ERC Starting Grant 337327 (NR), the Novo Nordisk Foundation (JBS) and the Independent Research Fund Denmark (SH, JBS).

Author contribution: Conceptualization IM and JBS; Investigation and/or Analysis SG, VS, SH, MS, MG, NS, NR, and IM; Writing: SG, JBS and IM (all coauthors contributed to the final ms).

Authors declare no competing financial interests.

\section{Figure Contribution: SG}

Main Figures: 1 (B-F), 2 (A-L, only analysis, recordings by $M G), 3(A-Q), 5(A-B$ together with VS, C-G, except Syt7 stainings), 6 (A-B, E-F), 7(A-H) and 8(A-B, E-F, G-N together with $S H)$.

Supplementary figures: S1 B, S2, S3 (A, C-G), S4, S5 (C-D), S6 (A-D,G) amd S7

\section{REFERENCES}

Anantharam, A., Bittner, M.A., Aikman, R.L., Stuenkel, E.L., Schmid, S.L., Axelrod, D., Holz, R.W. (2011) A new role for the dynamin GTPase in the regulation of fusion pore expansion. Mol Biol Cell 22(11):1907-18.

Bader, M.F., Holz, R.W., Kumakura, K., Vitale, N. (2002) Exocytosis: the chromaffin cell as a model system. Ann N Y Acad Sci 971:178-83.

Bai, J., Hu, Z., Dittman, J.S., Pym, E.C.G., and Kaplan, J.M. (2010). Endophilin Functions as a Membrane-Bending Molecule and Is Delivered to Endocytic Zones by Exocytosis. Cell 143, 430-441.

Borisovska, M., Zhao, Y., Tsytsyura, Y., Glyvuk, N., Takamori, S., Matti, U., Rettig, J., Südhof, T., Bruns, D. (2005). v-SNAREs control exocytosis of vesicles from priming to fusion. EMBO J. 24(12): 2114-2126. 
Boucrot, E., Ferreira, A.P.A., Almeida-Souza, L., Debard, S., Vallis, Y., Howard, G., Bertot, L., Sauvonnet, N., and McMahon, H.T. (2015). Endophilin marks and controls a clathrin-independent endocytic pathway. Nature 517, 460-465.

Chan, S.A., Doreian, B., Smith, C. (2010). Dynamin and myosin regulate differential exocytosis from mouse adrenal chromaffin cells. Cell Mol Neurobiol 30(8):1351-7.

Chen, H., Fre, S., Slepnev, V.I., Capua, M.R., Takei, K., Butler, M.H., Di Fiore, P.P., De Camilli, P. (1998). Epsin is an EH-domain-binding protein implicated in clathrin-mediated endocytosis. Nature 394(6695):793-7.

de Heuvel, E., Bell, A.W., Ramjaun, A.R., Wong, K., Sossin, W.S., and McPherson, P.S. (1997). Identification of the Major Synaptojanin-binding Proteins in Brain. J. Biol. Chem. 272, 8710-8716.

Elhamdani, A., Azizi, F., and Artalejo, C.R. (2006). Double Patch Clamp Reveals That Transient Fusion (Kiss-and-Run) Is a Major Mechanism of Secretion in Calf Adrenal Chromaffin Cells: High Calcium Shifts the Mechanism from Kiss-and-Run to Complete Fusion. J. Neurosci. 26, 3030-3036.

Farsi, Z., Gowrisankaran, S., Krunic, M., Rammner, B., Woehler, A., Lafer, E.M., Mim, C., Jahn, R., Milosevic, I. (2018). Clathrin coat controls synaptic vesicle acidification by blocking vacuolar ATPase activity. Elife 7. pii: e32569.

Farsad, K., Ringstad, N., Takei, K., Floyd, S.R., Rose, K., and Camilli, P.D. (2001). Generation of high curvature membranes mediated by direct endophilin bilayer interactions. J Cell Biol 155, 193-200.

Gerth, F., Jäpel, M., Pechstein, A., Kochlamazashvili, G., Lehmann, M., Puchkov, D., Onofri, F., Benfenati, F., Nikonenko, A.G., Fredrich, K., Shupliakov, O., Maritzen, T., Freund, C., Haucke, V. (2017). Intersectin associates with synapsin and regulates its nanoscale localization and function. Proc Natl Acad Sci USA 114(45), 12057-12062.

González-Jamett AM, Báez-Matus X, Hevia MA, Guerra MJ, Olivares MJ, Martínez AD, Neely A, Cárdenas AM (2010) The association of dynamin with synaptophysin regulates quantal size and duration of exocytotic events in chromaffin cells. J Neurosci 30(32):10683-91.

Gubar O, Morderer D, Tsyba L, Croisé P, Houy S, Ory S, Gasman S, Rynditch A (2013) Intersectin: The Crossroad between Vesicle Exocytosis and Endocytosis. Front Endocrinol (Lausanne) 4:109.

Houy, S., Croisé, P., Gubar, O., Chasserot-Golaz, S., Tryoen-Tóth, P., Bailly, Y., Ory, S., Bader, M.-F., and Gasman, S. (2013). Exocytosis and Endocytosis in Neuroendocrine Cells: Inseparable Membranes! Front. Endocrinol. 4.

Hua Y, Woehler A, Kahms M, Haucke V, Neher E, Klingauf J.(2013) Blocking endocytosis enhances short-term synaptic depression under conditions of normal availability of vesicles. Neuron 80(2):343-9. 
Hussain NK, Yamabhai M, Ramjaun AR, Guy AM, Baranes D, O’Bryan JP, Der CJ, Kay BK, McPherson PS. (1999) Splice variants of intersectin are components of the endocytic machinery in neurons and nonneuronal cells. J Biol Chem 274(22):15671-7.

Kedar, G.H., Munch, A.S., van Weering, J.R., Malsam, J., Scheutzow, A., de Wit, H., Houy, S., Tawfik, B., Söllner, T.H., Sørensen, J.B., Verhage, M. (2015). A Post-Docking Role of Synaptotagmin 1-C2B Domain Bottom Residues R398/399 in Mouse Chromaffin Cells. J. Neurosci. 35(42); 14172-14182.

Kirkham, M., Fujita, A., Chadda, R., Nixon, S.J., Kurzchalia, T.V., Sharma, D.K., Pagano, R.E., Hancock, J.F., Mayor, S., and Parton, R.G. (2005). Ultrastructural identification of uncoated caveolin-independent early endocytic vehicles. J. Cell Biol. 168, 465-476.

Kjaerulff, O., Brodin, L., and Jung, A. (2011). The Structure and Function of Endophilin Proteins. Cell Biochem. Biophys. 60, 137-154.

Koh TW, Verstreken P, Bellen HJ (2004) Dap160/intersectin acts as a stabilizing scaffold required for synaptic development and vesicle endocytosis. Neuron 43(2):193-205.

Kurps, J., Broeke, J.H., Cijsouw, T., Kompatscher, A., van Weering, J.R.T., and de Wit, H. (2014). Quantitative image analysis tool to study the plasma membrane localization of proteins and cortical actin in neuroendocrine cells. J. Neurosci. Methods 236, 1-10.

Liu, Y., Schirra, C., Stevens, D.R., Matti, U., Speidel, D., Hof, D., Bruns, D., Brose, N., Rettig, J. (2008). CAPS facilitates filling of the rapidly releasable pool of large dense-core vesicles. J Neurosci. 28(21):5594-601.

Malacombe, M., Ceridono, M., Calco, V., Chasserot-Golaz, S., McPherson, P.S., Bader, M.-F., and Gasman, S. (2006). Intersectin-1L nucleotide exchange factor regulates secretory granule exocytosis by activating Cdc42. EMBO J. 25, 3494-3503.

Man, K.N., Imig, C., Walter, A.M., Pinheiro, P.S., Stevens, D.R., Rettig, J., Sørensen, J.B., Cooper, B.H., Brose, N., Wojcik, S.M. (2015). Identification of a Munc13-sensitive step in chromaffin cell large dense-core vesicle exocytosis. Elife e10635.

Marie B, Sweeney ST, Poskanzer KE, Roos J, Kelly RB, Davis GW (2004) Dap160/intersectin scaffolds the periactive zone to achieve high-fidelity endocytosis and normal synaptic growth. Neuron 43(2):207-19.

Miki T, Malagon G, Pulido C, Llano I, Neher E, Marty A. (2016) Actin- and Myosin-Dependent Vesicle Loading of Presynaptic Docking Sites Prior to Exocytosis. Neuron 91(4):808-823.

Milosevic, I. (2018). Revisiting the Role of Clathrin-Mediated Endoytosis in Synaptic Vesicle Recycling. Front. Cell. Neurosci. 12.

Milosevic, I., Giovedi, S., Lou, X., Raimondi, A., Collesi, C., Shen, H., Paradise, S., O'Toole, E., Ferguson, S., Cremona, O., et al. (2011). Recruitment of Endophilin to Clathrin-Coated Pit Necks Is Required for Efficient Vesicle Uncoating after Fission. Neuron 72, 587-601. 
Milosevic, I., Sørensen, J.B., Lang, T., Krauss, M., Nagy, G., Haucke, V., Jahn, R., Neher, E. (2005). Plasmalemmal Phosphatidylinositol-4,5-Bisphosphate Level Regulates the Releasable Vesicle Pool Size in Chromaffin Cells. J. Neurosci. 25, 2557-2565.

Momboisse F, Ory S, Ceridono M, Calco V, Vitale N, Bader MF, Gasman S. (2010) The Rho guanine nucleotide exchange factors Intersectin $1 \mathrm{~L}$ and $\beta$-Pix control calcium-regulated exocytosis in neuroendocrine PC12 cells. Cell Mol Neurobiol 30(8):1327-33.

Momboisse, F., Houy, S., Ory, S., Calco, V., Bader, M.-F., and Gasman, S. (2011). How important are Rho GTPases in neurosecretion? J. Neurochem. 117, 623-631.

Mosharov, E.V., Sulzer, D. (2005). Analysis of exocytotic events recorded by amperometry. Nat. Methods 2(9): 651-658

Murdoch, J.D., Rostosky, C.M., Gowrisankaran, S., Arora, A.S., Soukup, S.-F., Vidal, R., Capece, V., Freytag, S., Fischer, A., Verstreken, P., et al. (2016). Endophilin-A Deficiency Induces the Foxo3a-Fbxo32 Network in the Brain and Causes Dysregulation of Autophagy and the UbiquitinProteasome System. Cell Rep. 17, 1071-1086.

Nadif Kasri, N., Nakano-Kobayashi, A., and Van Aelst, L. (2011). Rapid Synthesis of the X-Linked Mental Retardation Protein OPHN1 Mediates mGluR-Dependent LTD through Interaction with the Endocytic Machinery. Neuron 72, 300-315.

Nagy, G., Milosevic, I., Fasshauer, D., Müller, M., de Groot, B., Lang, T., Wilson, M.C., Sørensen, J.B. (2005) Alternative splicing of SNAP-25 regulates secretion through non-conservative substitutions in the SNARE domain. Mol Biol Cell 16, 5675-85.

Nakano-Kobayashi, A., Kasri, N.N., Newey, S.E., and Aelst, L.V. (2009). The Rho-Linked Mental Retardation Protein OPHN1 Controls Synaptic Vesicle Endocytosis via Endophilin A1. Curr. Biol. 19, 1133-1139.

Neher, E. (2006). A comparison between exocytic control mechanisms in adrenal chromaffin cells and a glutamatergic synapse. Pflüg. Arch. 453, 261-268.

Okamoto M, Schoch S, Südhof TC (1999) EHSH1/intersectin, a protein that contains EH and SH3 domains and binds to dynamin and SNAP-25. A protein connection between exocytosis and endocytosis? J Biol Chem 274(26):18446-54

Park, Y., Hernandez, J.M., van den Bogaart, G., Ahmed, S., Holt, M., Riedel, D., and Jahn, R. (2012). Controlling synaptotagmin activity by electrostatic screening. Nat. Struct. Mol. Biol. 19, 991-997.

Pechstein, A., Bacetic, J., Vahedi-Faridi, A., Gromova, K., Sundborger, A., Tomlin, N., Krainer, G., Vorontsova, O., Schäfer, J.G., Owe, S.G., et al. (2010). Regulation of synaptic vesicle recycling by complex formation between intersectin 1 and the clathrin adaptor complex AP2. Proc. Natl. Acad. Sci. 107, 4206-4211. 
Pechstein, A., Gerth, F., Milosevic, I., Jäpel, M., Eichhorn-Grünig, M., Vorontsova, O., Bacetic, J., Maritzen, T., Shupliakov, O., Freund, C., et al. (2015). Vesicle uncoating regulated by SH3-SH3 domain-mediated complex formation between endophilin and intersectin at synapses. EMBO Rep. 16, 232-239.

Perera, R.M., Zoncu, R., Lucast, L., Camilli, P.D., and Toomre, D. (2006). Two synaptojanin 1 isoforms are recruited to clathrin-coated pits at different stages. Proc. Natl. Acad. Sci. 103, $19332-19337$.

Pinheiro, P.S., Jansen, A.M., de Wit, H., Tawfik, B., Madsen, K.L., Verhage, M., Gether, U., Sørensen, J.B. (2014). The BAR domain protein PICK1 controls vesicle number and size in adrenal chromaffin cells. J. Neurosci. 34(32): 10688-10700

Renard, H.-F., Simunovic, M., Lemière, J., Boucrot, E., Garcia-Castillo, M.D., Arumugam, S., Chambon, V., Lamaze, C., Wunder, C., Kenworthy, A.K., et al. (2015). Endophilin-A2 functions in membrane scission in clathrin-independent endocytosis. Nature 517, 493-496.

Revelo, N.H., and Rizzoli, S.O. (2016). The Membrane Marker mCLING Reveals the Molecular Composition of Trafficking Organelles. Curr. Protoc. Neurosci. 74, 2.25.1-2.25.21.

Ringstad, N., Nemoto, Y., and De Camilli, P. (1997). The SH3p4/Sh3p8/SH3p13 protein family: Binding partners for synaptojanin and dynamin via a Grb2-like Src homology 3 domain. Proc. Natl. Acad. Sci. 94, 8569-8574.

Ringstad, N., Gad, H., Löw, P., Di Paolo, G., Brodin, L., Shupliakov, O., De Camilli P. (1999). Endophilin/SH3p4 is required for the transition from early to late stages in clathrin-mediated synaptic vesicle endocytosis. Neuron 24(1): 143-154

Ringstad N, Nemoto Y, De Camilli P. (2001). Differential expression of endophilin 1 and 2 dimers at central nervous system synapses. J. Biol. Chem. 276(44): 40424-40430.

Sakaba, T., Kononenko, N.L., Bacetic, J., Pechstein, A., Schmoranzer, J., Yao, L., Barth, H., Shupliakov, O., Kobler, O., Aktories, K., et al. (2013). Fast neurotransmitter release regulated by the endocytic scaffold intersectin. Proc. Natl. Acad. Sci. U. S. A. 110, 8266-8271.

Schuske, K.R., Richmond, J.E., Matthies, D.S., Davis, W.S., Runz, S., Rube, D.A., van der Bliek, A.M., and Jorgensen, E.M. (2003). Endophilin Is Required for Synaptic Vesicle Endocytosis by Localizing Synaptojanin. Neuron 40, 749-762.

Schonn, J.S., Maximov, A., Lao, Y., Südhof, T.C., Sørensen, J.B. (2008). Synaptotagmin-1 and 7 are functionally overlapping $\mathrm{Ca} 2+$ sensors for exocytosis in adrenal chromaffin cells. Proc. Natl. Acad. Sci. U.S.A. 105(10): 3998-4003

Simpson F, Hussain NK, Qualmann B, Kelly RB, Kay BK, McPherson PS, Schmid SL (1999) SH3domain-containing proteins function at distinct steps in clathrin-coated vesicle formation. Nat Cell Biol 1(2):119-24. 
Simunovic, M., Manneville, J.-B., Renard, H.-F., Evergren, E., Raghunathan, K., Bhatia, D., Kenworthy, A.K., Voth, G.A., Prost, J., McMahon, H.T., et al. (2017). Friction Mediates Scission of Tubular Membranes Scaffolded by BAR Proteins. Cell 170, 172-184.e11.

Smith, C., and Neher, E. (1997). Multiple Forms of Endocytosis In Bovine Adrenal Chromaffin Cells. J. Cell Biol. 139, 885-894.

Sørensen, J.B., Nagy, G., Varoqueaux, F., Nehring, R.B., Brose, N., Wilson, M.C., and Neher, E. (2003). Differential Control of the Releasable Vesicle Pools by SNAP-25 Splice Variants and SNAP-23. Cell 114, 75-86.

Takei, K., Slepnev, V.I., Haucke, V., and De Camilli, P. (1999). Functional partnership between amphiphysin and dynamin in clathrin-mediatedendocytosis. Nat. Cell Biol. 1, 33-39.

Trifaró JM1, Rodríguez del Castillo A, Vitale ML. (1992) Dynamic changes in chromaffin cell cytoskeleton as prelude to exocytosis. Mol Neurobiol 6(4):339-58.

Vinatier J, Herzog E, Plamont MA, Wojcik SM, Schmidt A, Brose N, Daviet L, El Mestikawy S, Giros B (2006) Interaction between the vesicular glutamate transporter type 1 and endophilin A1, a protein essential for endocytosis. J Neurochem 97(4):1111-25.

Verstreken, P., Koh, T.-W., Schulze, K.L., Zhai, R.G., Hiesinger, P.R., Zhou, Y., Mehta, S.Q., Cao, Y., Roos, J., and Bellen, H.J. (2002). Endophilin mutations block clathrin-mediated endocytosis but not neurotransmitter release. Cell 109(1): 101-112

Voglmaier SM, Kam K, Yang H, Fortin DL, Hua Z, Nicoll RA, Edwards RH (2006) Distinct endocytic pathways control the rate and extent of synaptic vesicle protein recycling. Neuron 51(1):71-84.

Watanabe, S., Mamer, L.E., Raychaudhuri, S., Luvsanjav, D., Eisen, J., Trimbuch, T., SöhlKielczynski, B., Fenske, P., Milosevic, I., Rosenmund, C., et al. (2018). Synaptojanin and Endophilin Mediate Neck Formation during Ultrafast Endocytosis. Neuron 98, 1184-1197.e6.

Winther AM, Jiao W, Vorontsova O, Rees KA, Koh TW, Sopova E, et al. (2013) The dynaminbinding domains of Dap160/intersectin affect bulk membrane retrieval in synapses. J Cell Sci 126 (Pt 4):1021-31.

Wu LG, Hamid E, Shin W, Chiang HC (2014) Exocytosis and endocytosis: modes, functions, and coupling mechanisms. Annu Rev Physiol 76, 301-31.

Yamabhai M, Hoffman NG, Hardison NL, McPherson PS, Castagnoli L, Cesareni G, Kay BK. (1998) Intersectin, a novel adaptor protein with two Eps15 homology and five Src homology 3 domains. J Biol Chem 273(47):31401-7.

Yu Y, Chu PY, Bowser DN, Keating DJ, Dubach D, Harper I, Tkalcevic J, Finkelstein DI, Pritchard MA (2009) Mice deficient for the chromosome 21 ortholog Itsn1 exhibit vesicle-trafficking abnormalities. Hum Mol Genet 17(21), 3281-90. 
MAIN FIGURES 
A

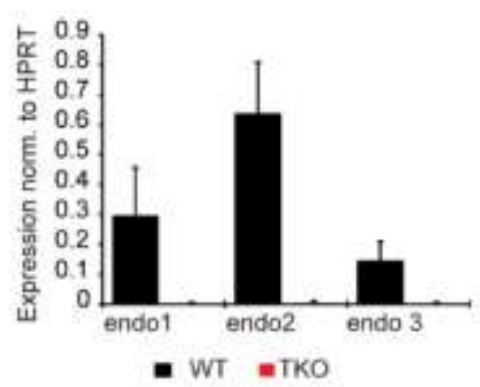

C
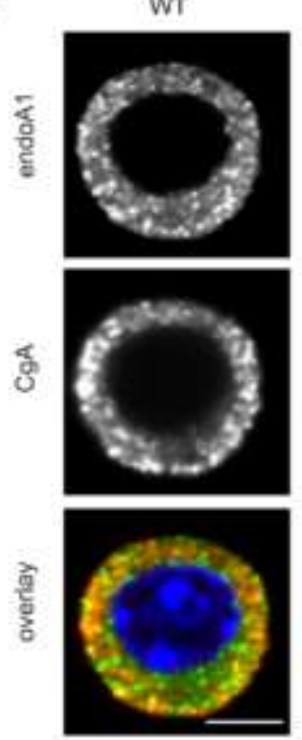

E

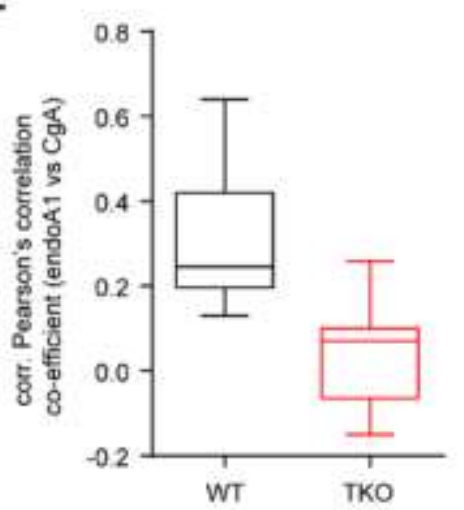

B

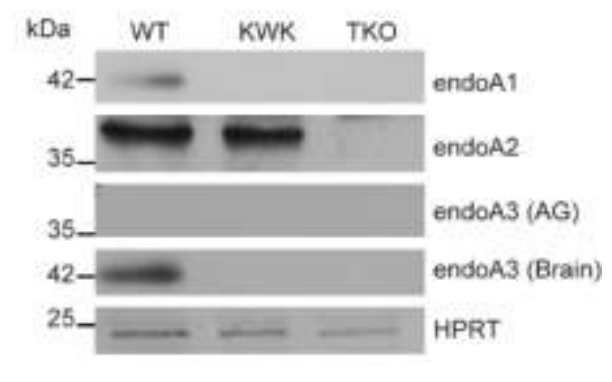

D
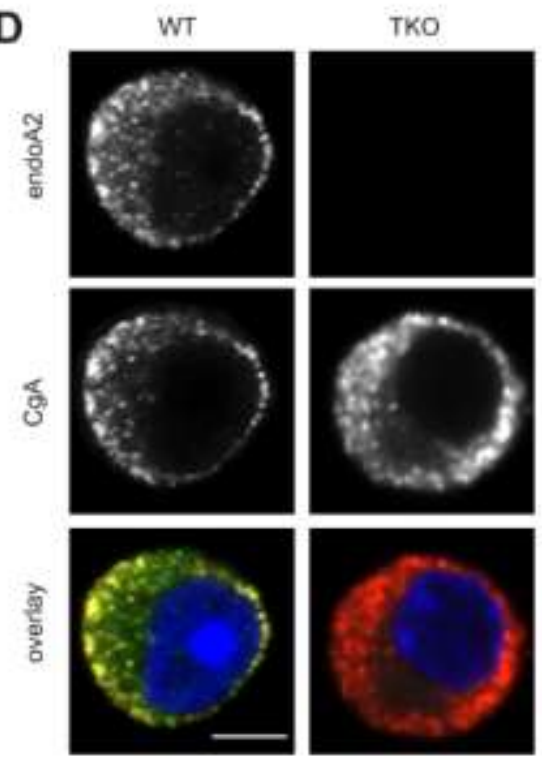

$\mathbf{F}$

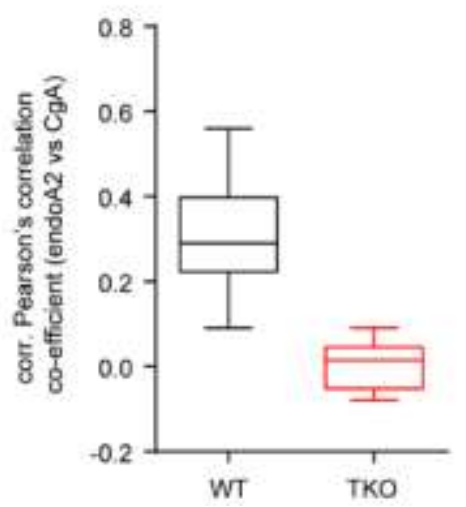

Figure 1. Endophilins are present in adrenal chromaffin cells and enriched at the neurosecretory vesicles

(A) Real-time PCR for endophilin 1, 2 and 3 performed on RNA isolated from the cells at the center of adrenal gland (medulla) showed the presence of all three endophilin's mRNAs. (B) Western blot analysis 
of adrenal gland lysate blotted with anti-endophilin 1, 2 and 3 antibodies. Endophilin 3 could not be detected in the adrenal gland homogenate, although it could be detected in the same amount of WT brain sample. (C-D) Confocal images of WT and endophilin TKO mouse chromaffin cell stained with endophilin 1 (C) and endophilin 2 (D), and co-labeled with chromogranin-A (CgA), a LDCV marker. The images show an optical section through the cell's equatorial plane. Scale: $3 \mu \mathrm{m}$. The endophilin antibodies used were characterized as specific since little to none signal could be detected in endophilin TKO cells. (E-F) Colocalization analysis of $\mathrm{CgA}$ with endophilin 1 and endophilin 2, respectively, was performed in WT and endophilin TKO cells as detailed in Methods (note that the accidental colocalization was subtracted, resulting in negative correlation in some endophilin TKO cells). 
A
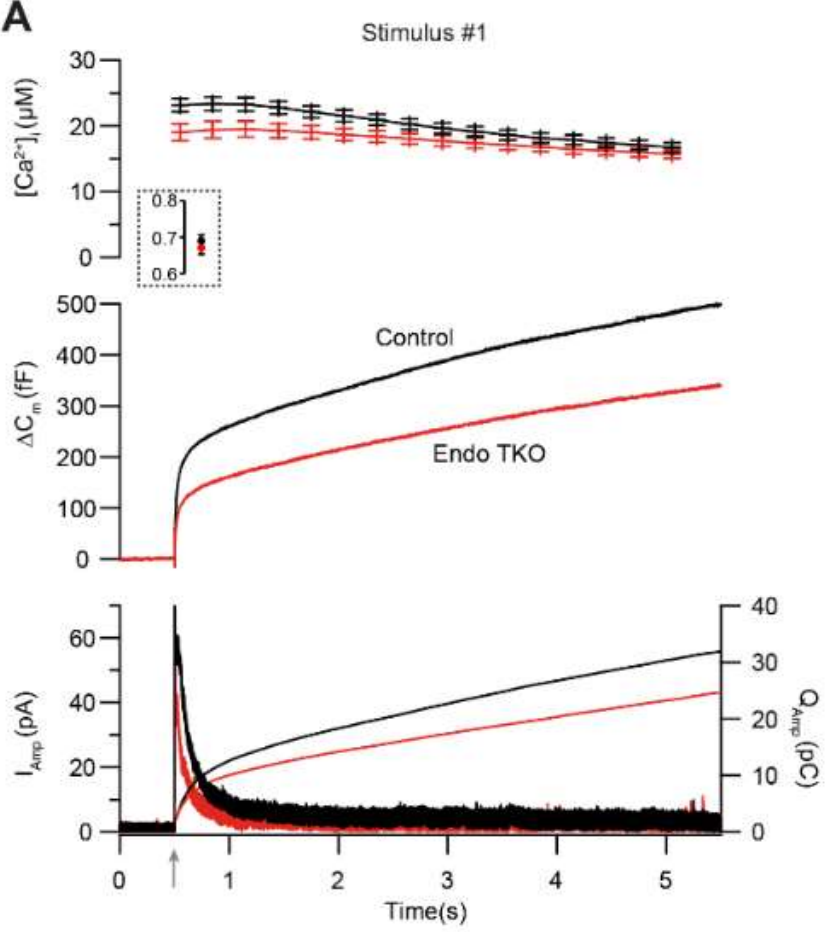

B

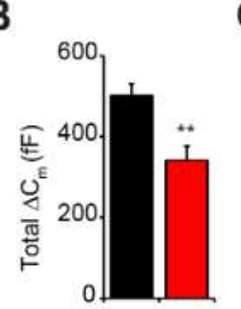

E

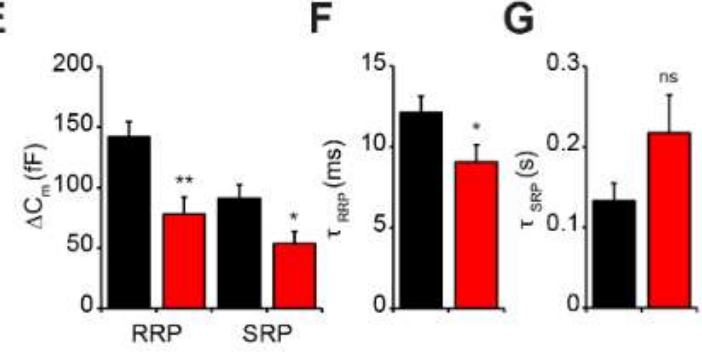

H
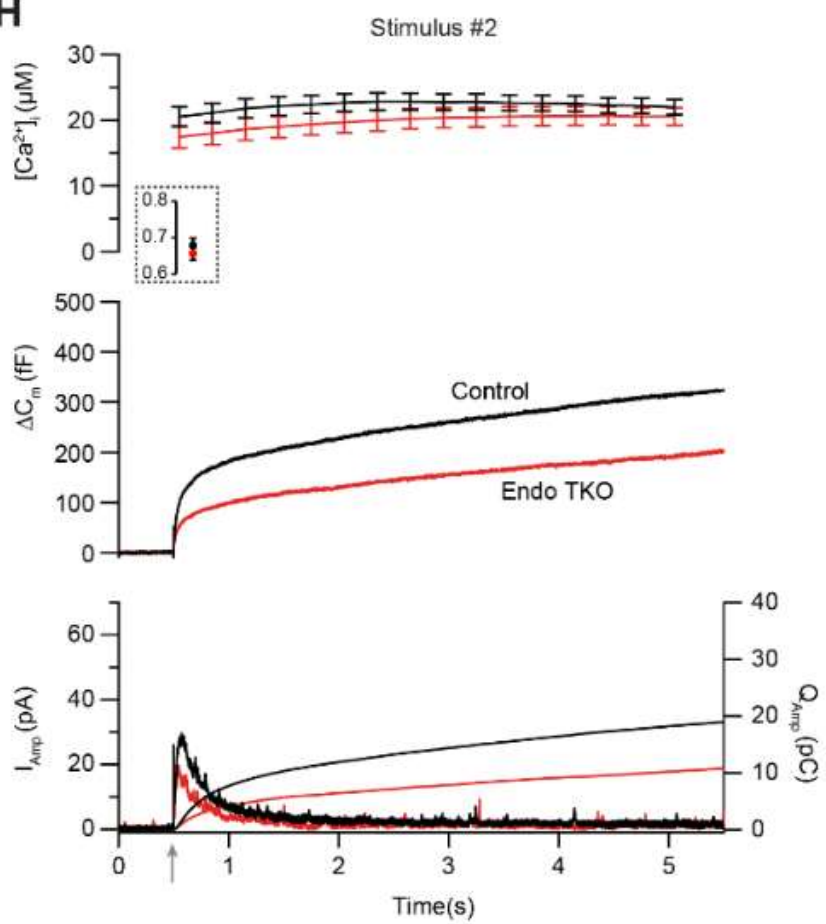

I

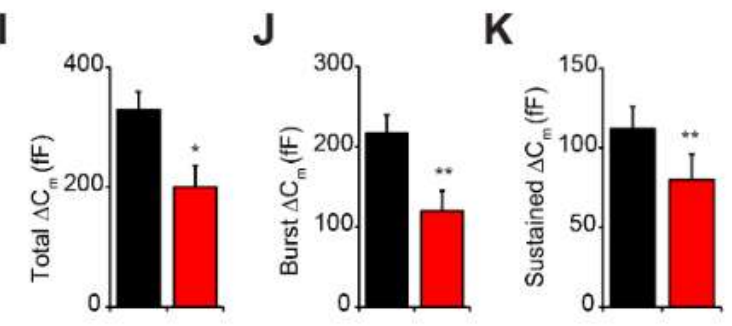

L

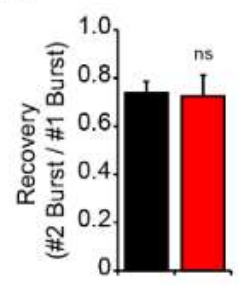

Figure 2. Lack of endophilins reduces exocytosis in mouse chromaffin cells 
(A) Exocytosis induced by the UV-flash photolysis of caged calcium (stimulus \#1, at arrow) was reduced in endophilin TKO chromaffin cells (red traces) compared to littermate KOWTKO control cells (black traces). (A) Top: intracellular calcium level increase induced by flash photolysis at $0.5 \mathrm{~s}$ (at arrow). The inset shows the pre-flash calcium levels in the cells. Middle: averaged traces of membrane capacitance changes upon $\mathrm{Ca}^{2+}$-induced exocytosis. Bottom: mean amperometric current (left axis) and cumulative charge (right axis). (B-D) Analysis of capacitance traces (30 control cells - black bars, 29 TKO cells - red bars) revealed an overall reduction of exocytosed vesicles. Exponential fitting of the capacitance traces revealed changes in burst (exocytosis within the first 1s; C) and sustained phase of release (D). (E) Both RRP and SRP components of the burst phase were significantly reduced in endophilin TKO cells. (F) Fusion kinetics of RRP vesicles was faster in the TKO cells (unpaired t-test). (G) Although on average slower, fusion kinetics constant of SRP was not significantly changed in endophilin TKO cells (unpaired t-test). (H-K) Exocytosis induced by a second stimulus, elicited 100 s after the first stimulus, showed similar reduction in total vesicle exocytosed as well as burst and sustained components of the release (the smaller exocytic responses prevented exponential kinetic analysis). Panels in (H) are arranged as detailed in (A). (L) Measure of recovery, calculated as the ratio of burst secretion of the second over the first stimulus was not significantly altered. 
A

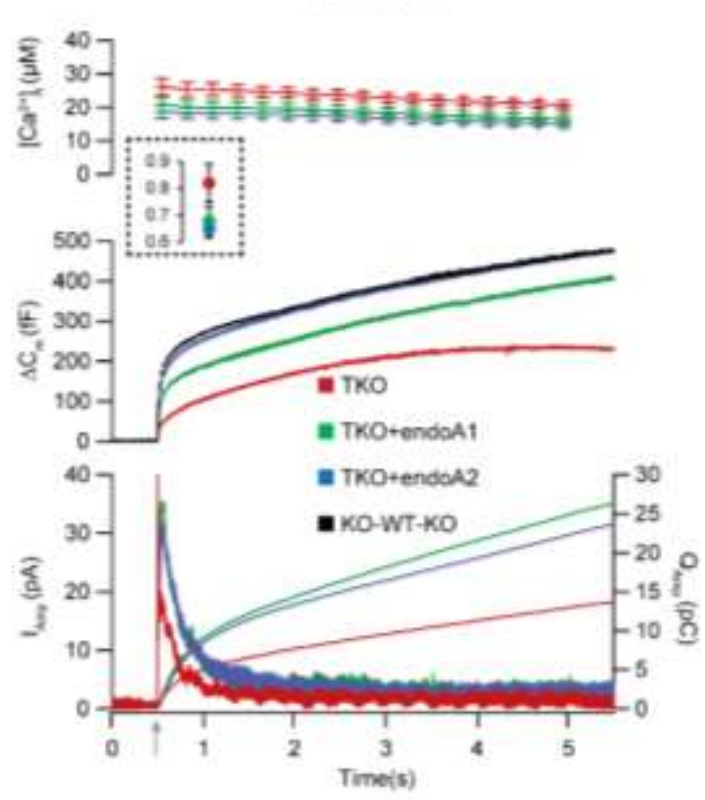

H

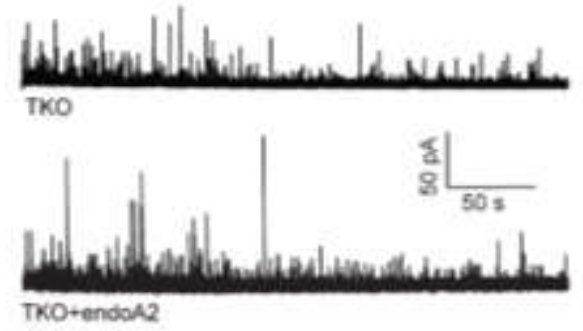

I

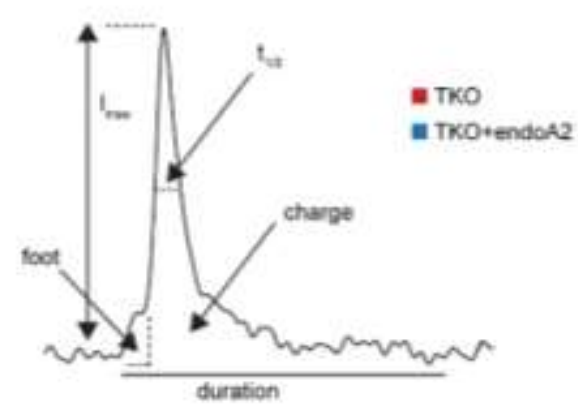

B

C
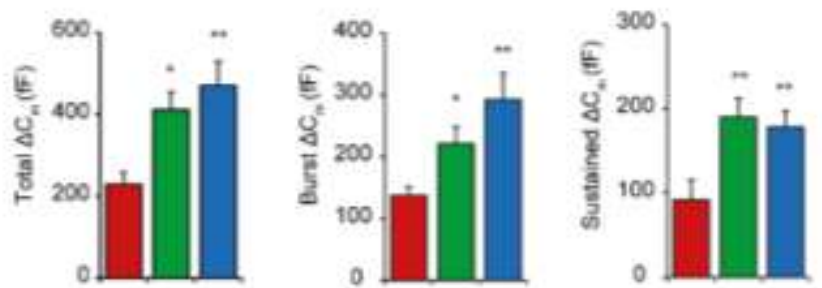

E

$\mathbf{F}$

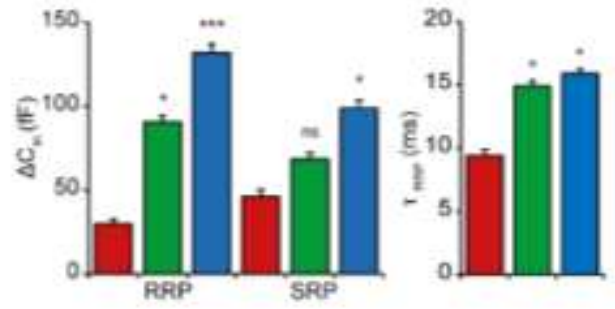

G

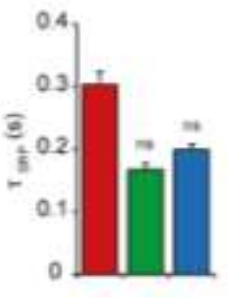

J

K

L

M
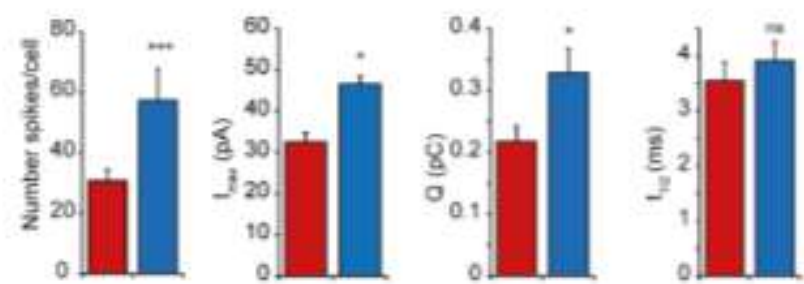

N

0

$\mathbf{P}$

Q
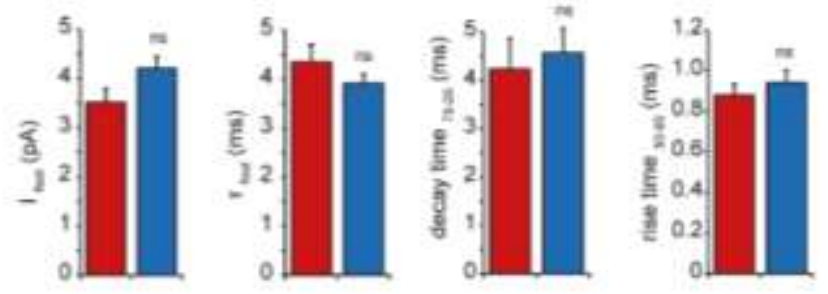

Figure 3. Expression of endophilin 1 and endophilin 2 rescued exocytosis in endophilin TKO cells 
(A-B) Expression of endophilin 1 and endophilin 2 using bicistronic lentiviral system rescued the exocytic defects seen in endophilin TKO cells. Panel arranged as in Figure 2A, with 3 groups: endophilin TKO (red traces; mean of 24 cells), endophilin TKO + endophilin 1 (green traces; mean of 23 cells) and endophilin TKO + endophilin 2 (blue traces; mean of 25 cells). Endophilin KOWTKO data from Figure 2A (black trace; mean of 30 cells) are superimposed. Note that both endophilin 1 and endophilin 2 can rescue exocytosis, rescue with endophilin 2 is indistinguishable from control KOWTKO cells. (C-E) Burst and sustained component, as well as RRP size, were rescued upon expression of endophilin 1 and 2, respectively. (F-G) The altered kinetics of RRP in TKO was rescued (F), while time constant of SRP was not significantly changed (G) upon expression of endophilin 1 and 2, respectively (Kruskal-Wallis test with Dunn's multiple comparison test). (H) Exemplary traces from amperometric recordings of endophilin TKO and endophilin TKO expressing endophilin 2. (I) Schematic of analyzed amperometric spike parameters. (J-Q) Amperometry analysis reveals problems in vesicle fusion: number of fusion events per cell (I), single spike amplitude $(\mathrm{J})$ and charge (K) were significantly decreased in endophilin TKO cells, while the rise time (P) and foot properties (N-Q) were unaltered (Mann-Whitney test). 

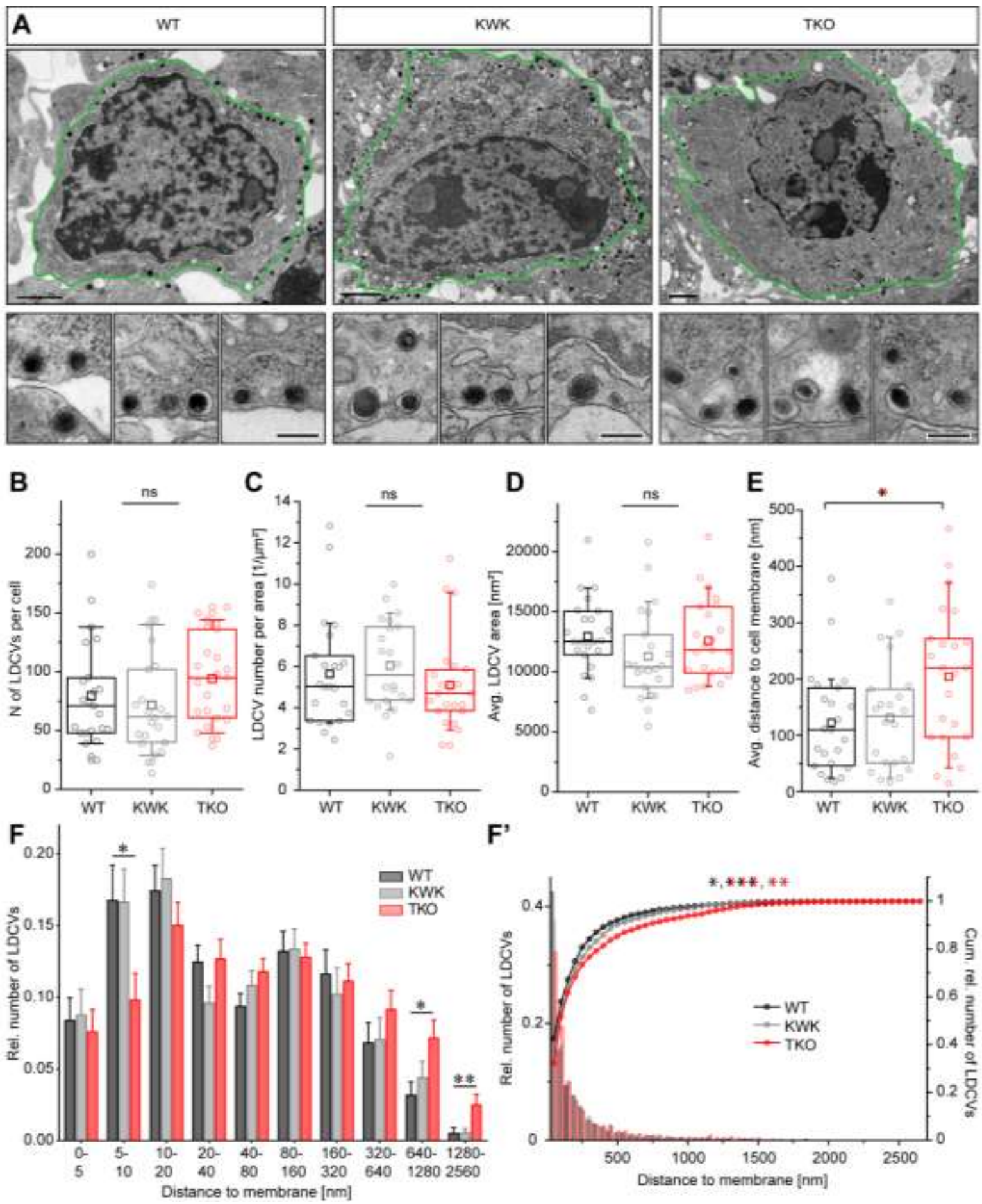

$\mathbf{F}^{\prime}$

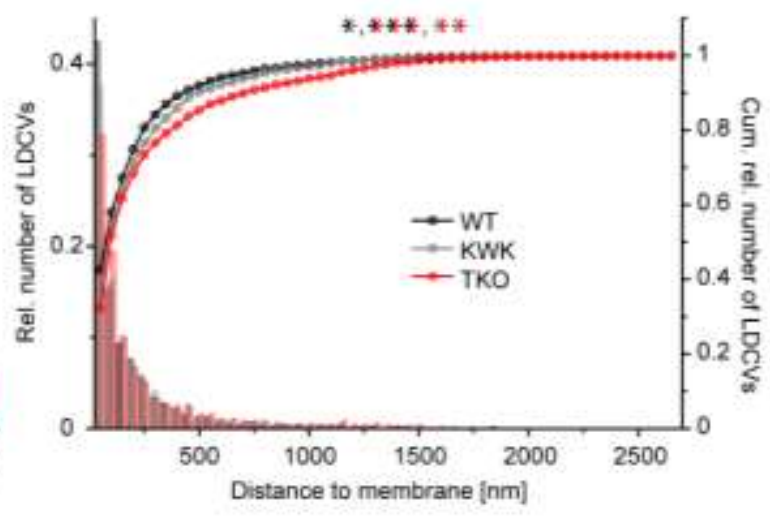

Figure 4. Number and size of LDCVs were not altered in endophilin TKO chromaffin cells, but less LDCVs was found in the plasma membrane proximity 
(A) Example images of WT, endophilin KOWTKO (littermate control) and endophilin TKO chromaffin cells in the adrenal gland. Green line highlights one cell in the image. Note that the TKO cells is shown at lower magnification so the whole cell can be displayed. Panels below show higher magnification of LDCVs in the proximity of the plasma membrane (three examples are shown). Note that some LDCVs in endophilin TKO cells were not as close to the plasma membrane as in littermate and WT controls. Scale bar $100 \mathrm{~nm}$. (B-C) The total number of LDCVs per cell, and cell area, was unchanged between endophilin TKO and the control cells (one-way ANOVA). (D) The average LDCV area was unchanged between endophilin TKO and the control cells (one-way ANOVA). (E) The average distance of LDCVs from the plasma membrane was increased in cells without endophilins (one-way ANOVA followed by Tukey's post-hoc test). (F) Distances between the LDCV membrane and the plasma membrane, after being normalized per cell. WT - black, endophilin KOWTKO - gray, endophilin TKO - red; at least 23 cells from 4 different animals and independent embeddings per group (Kolmogorov-Smirnov test with Bonferroni-correction). ( $F^{\prime}$ ) Relative frequency distribution and cumulative plots produced by binning all vesicles revealed altered distribution of LDCVs in endophilin TKO cells (Kolmogorov-Smirnov test with Bonferroni-correction). 
A

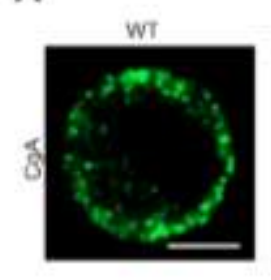

C
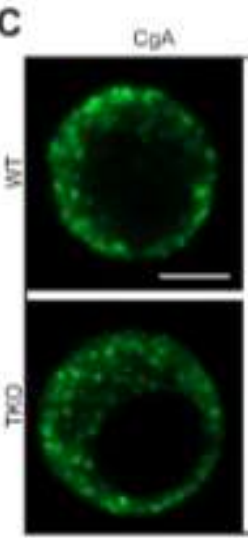

$\mathbf{F}$
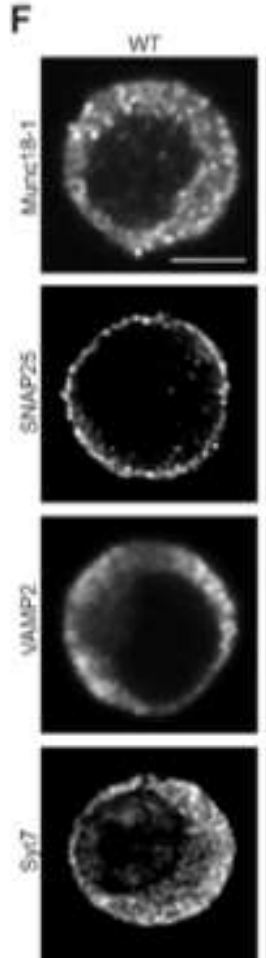

G
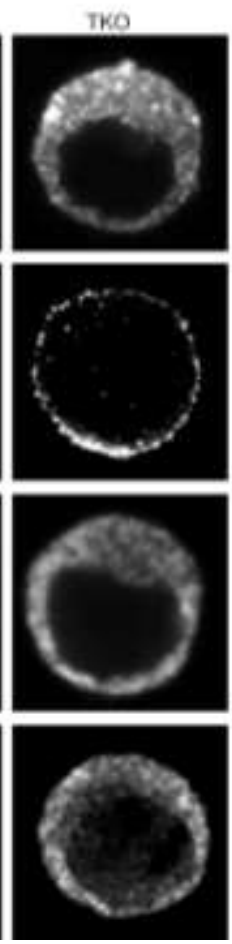
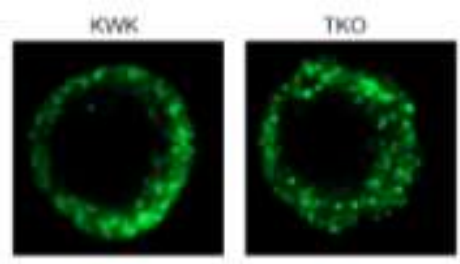

\section{B}

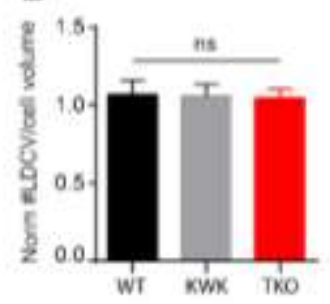

D
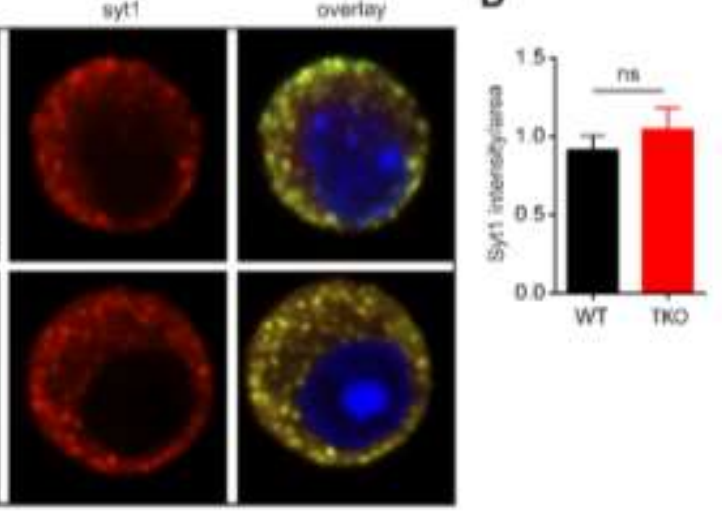

E

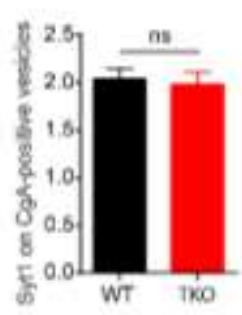

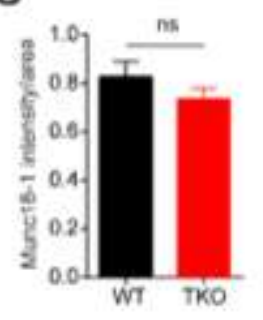
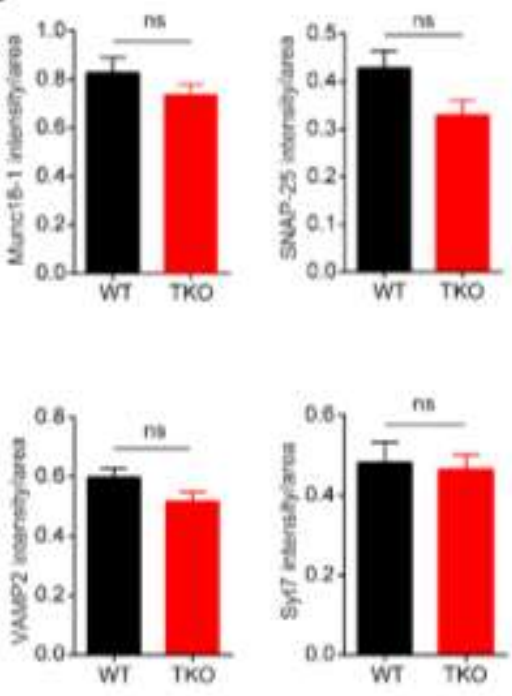

Figure 5. Exocytic machinery is unaltered in the chromaffin cells without endophilin 
(A-B) Confocal image of endophilin TKO and control (WT and littermate endophilin KOWTKO) chromaffin cells stained with chromogranin-A (CgA; LDCV marker) revealed no difference in the number of $\mathrm{CgA}$ positive vesicles measured in the whole volume of the cell (40 cells, $p$-value $=0.96$, one-way ANOVA with Tukey's multiple comparison). An optical section through the equatorial plane of the cells is shown. (C) Images of endophilin TKO, littermate KOWTKO and WT chromaffin cells stained with anti-synaptotagmin1 (Syt-1) and $\mathrm{CgA}$ antibodies. (D) Immunofluorescence levels revealed no change in synaptotagmin-1 levels ( 40 cells, $p$-value $=0.45$, unpaired t-test). (E) Quantification of the Syt- 1 intensity on the CgA-positive vesicles revealed no significant difference between the genotypes ( 40 cells, $p$-value $=0.76$, unpaired t-test). (F) Representative images of endophilin TKO and WT chromaffin cell stained against Munc18-1, SNARE proteins SNAP25 and VAMP2 and synaptotagmin-7 shown no difference in the distribution of aforementioned proteins. (G) Quantification of Munc18-1 (30 cells, p-value $=0.25$, unpaired t-test), SNAP25 ( 30 cells, $p$-value $=0.07$, unpaired $t$-test), VAMP- 2 ( 40 cells, $p$-value $=0.27$, unpaired t-test) and Syt7 (30 cells, $p$-value $=0.06$, unpaired t-test) immunofluorescence showed no significant changes for any of these proteins. Scale bar: $3 \mu \mathrm{m}$. 
A
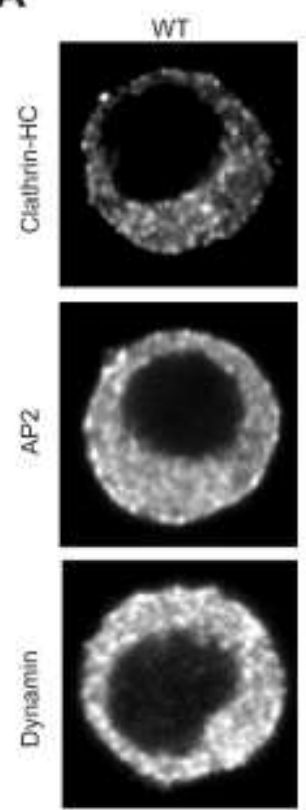

C
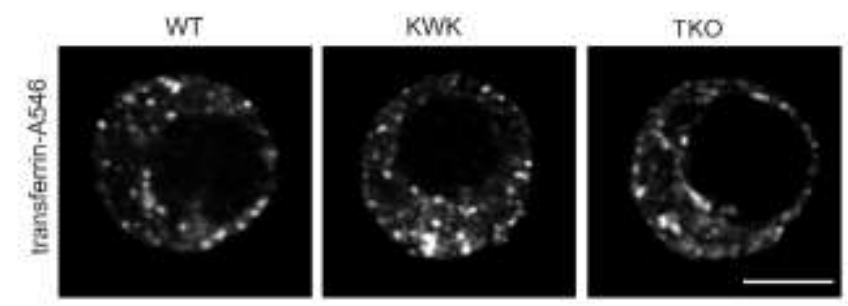

E

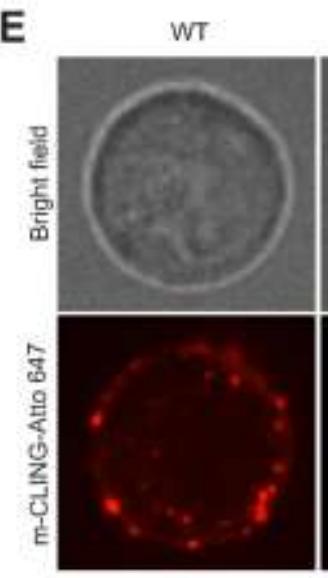

KWK
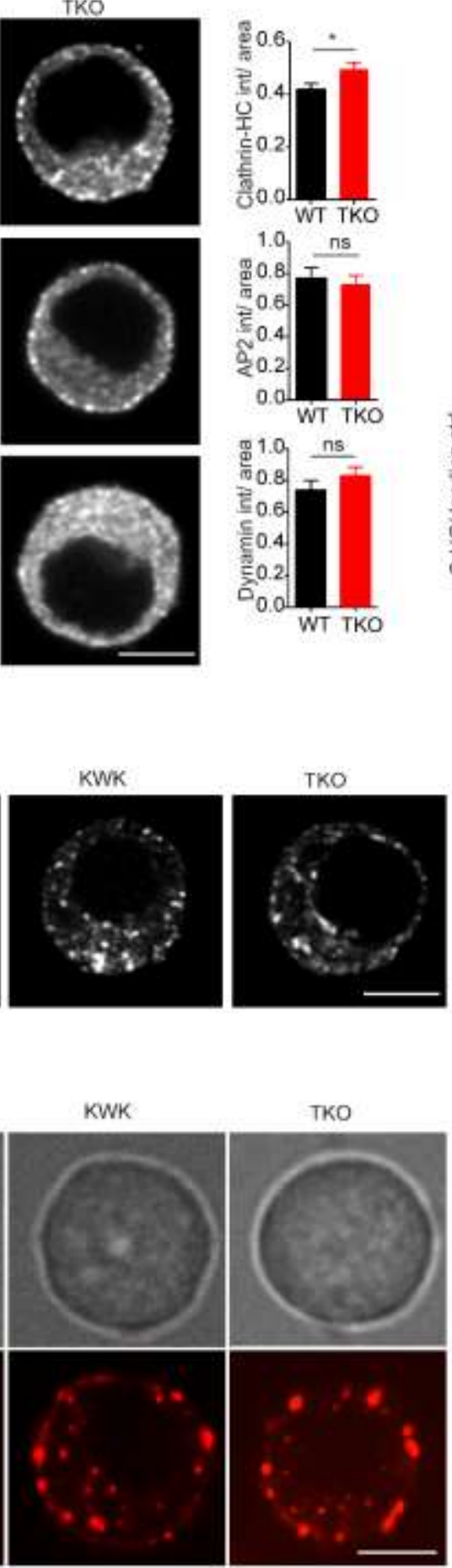
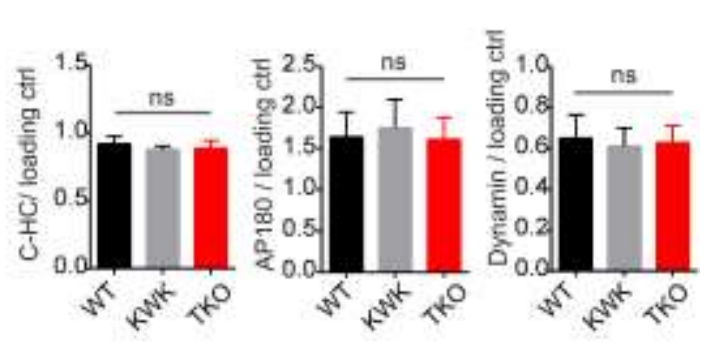

D

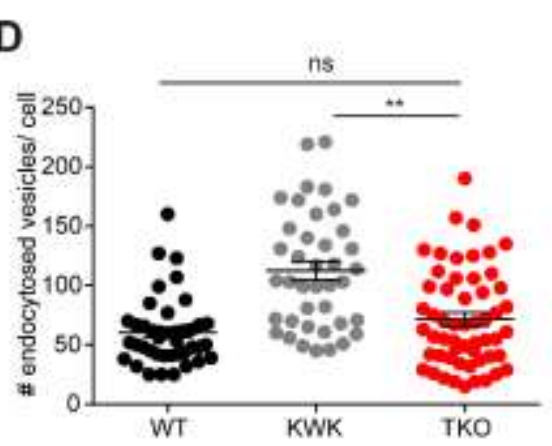

$\mathbf{F}$

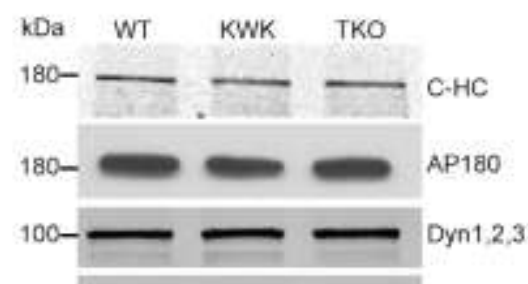

$25-20$ HPRT

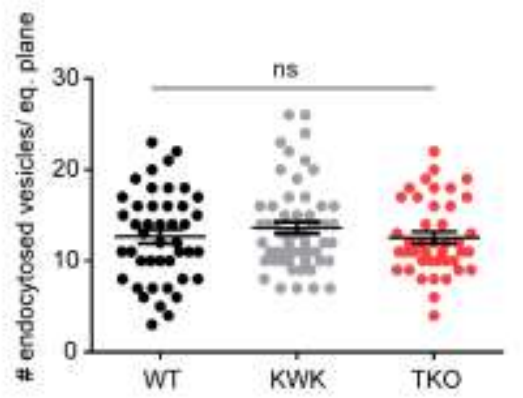

Figure 6. Endocytic defects in the absence of endophilin in chromaffin cells 
(A) Immunofluorescence against clathrin heavy chain (HC), adaptor protein 2 (AP2) and dynamin-1 in WT and endophilin TKO cells. Fluorescence quantification revealed small but significant increase in clathrin $\mathrm{HC}$ intensities, whereas protein levels of AP2 and dynamin-1 were unaltered in endophilin TKO cells. (B) Proteins levels of main endocytic factors - clathrin, adaptor protein 180 (AP180) and dynamins 1-3 (inspected by Western blotting) were not altered in adrenal gland homogenates. Below: Quantification from 3-5 independent experiments. (C-D) Transferrin (conjugated with Alexa Fluor-546) uptake revealed a reduction in constitutive endocytosis in the endophilin TKO cells compared to the littermate control ( $p$ value $=0.001$, one-way ANOVA, 40 cells; note that an uptake in the whole cell was analyzed). (E-F) mClingAtto647 uptake by chromaffin cells of the indicated genotypes showed no significant difference in the number of endocytosed vesicles ( $p$-value $=0.44$, one-way ANOVA, 42 cells; note that an uptake in only one cell plane was analyzed). The specificity of mCling-Atto647 uptake was tested in the stimulated cells in the presence of Pitstop-2 inhibitor (Suppl data S5). 
A

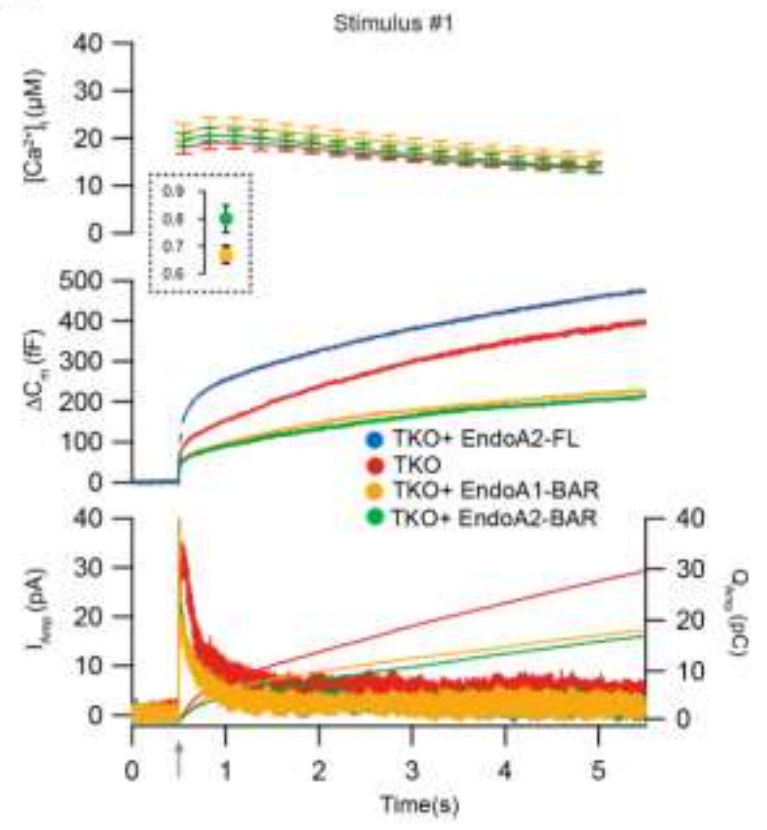

B

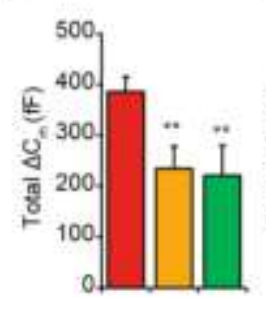

C

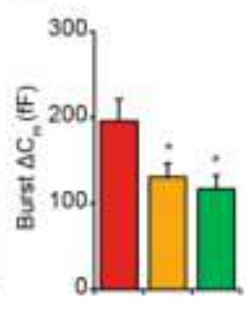

D

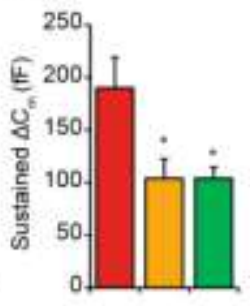

E
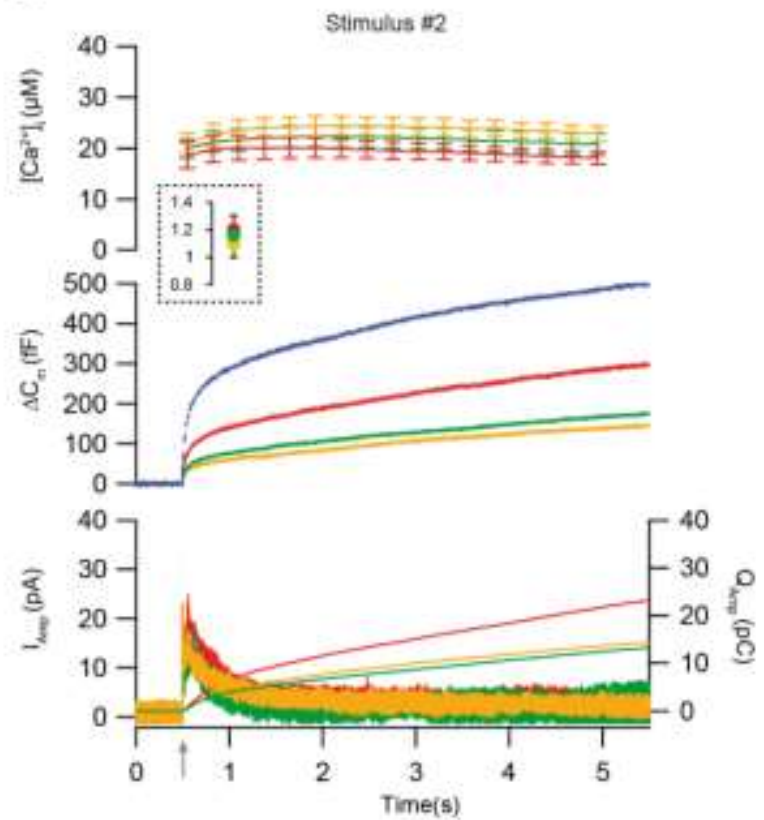

F

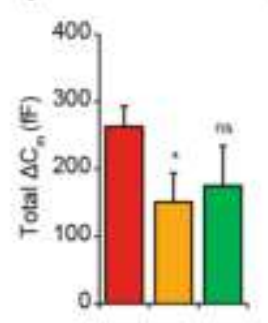

G

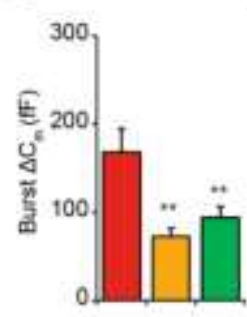

H

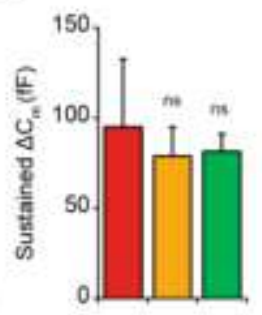

I

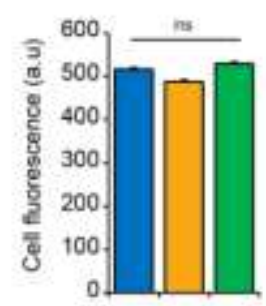

Figure 7. Endophilin 1 BAR and endophilin 2 BAR-domains are not sufficient to mediate exocytic release from chromaffin cells 
(A) Exocytosis induced by calcium uncaging in endophilin TKO chromaffin cells compared to TKO cells expressing either endophilin 1 BAR or endophilin 2 BAR domain. Top: intracellular calcium level increase induced by flash photolysis at $0.5 \mathrm{~s}$ (at arrow). The inset shows the pre-flash calcium levels. Middle: averaged traces of membrane capacitance upon $\mathrm{Ca}^{2+}$-induced exocytosis. Bottom: mean amperometric current (left axis) and cumulative charge (right axis). (C-H) Quantification of changes in capacitance revealed a further reduction in different phases of release - capacitance at $1 \mathrm{~s}$ (burst) and $4.5 \mathrm{~s}$ (total) in TKO cells expressing endophilin 1 BAR or endophilin 2 BAR domain ( 20 cells, one-way ANOVA with Tukey's multiple comparison test). (I) Fluorescence intensities measured from cells expressing endophilin 2 (FL), endophilin 1 BAR or endophilin 2 BAR domain were comparable (48 cells). 
A
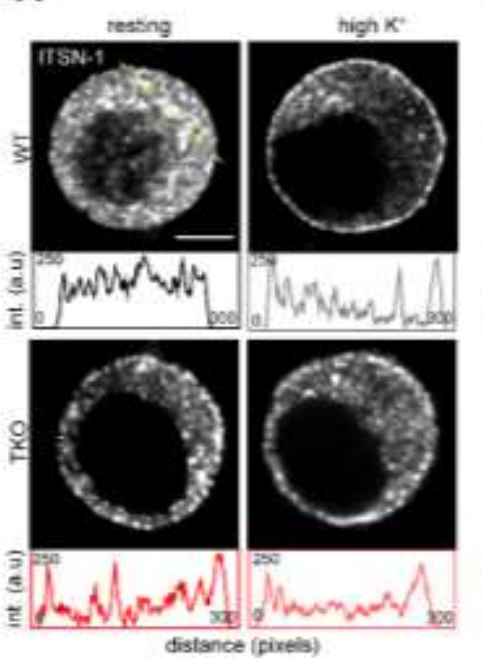

B

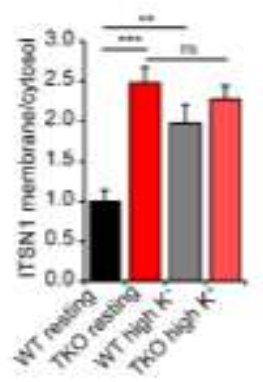

G

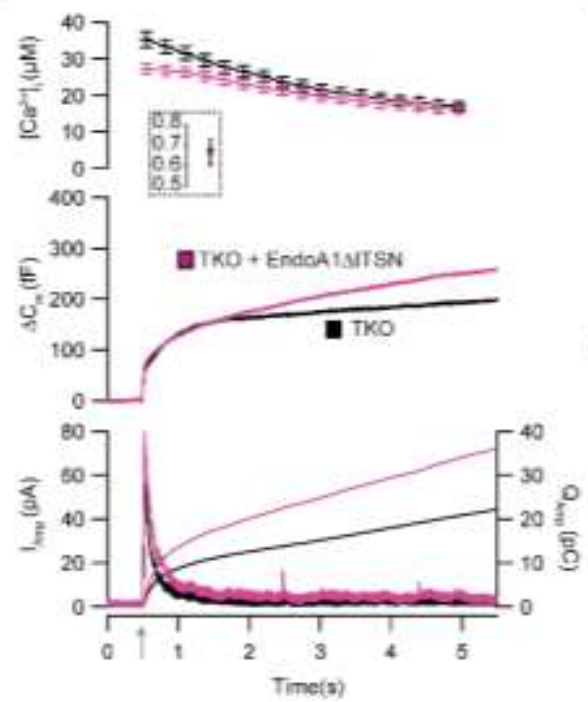

C
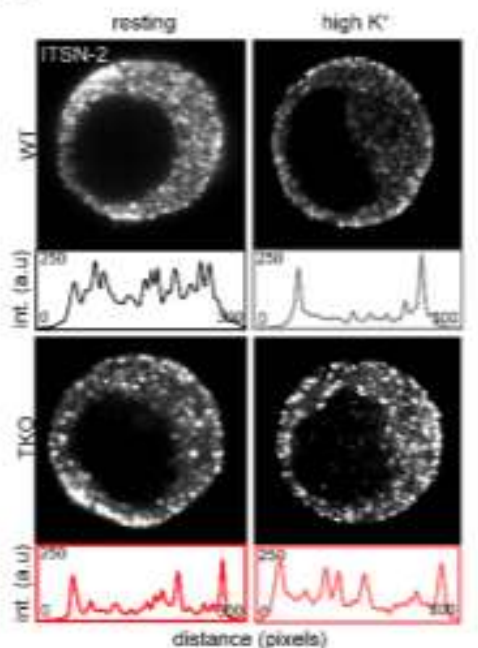

D

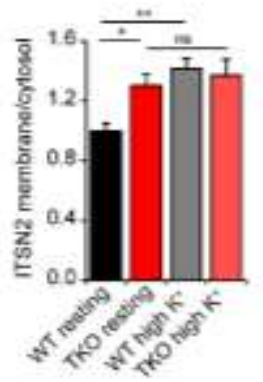

E
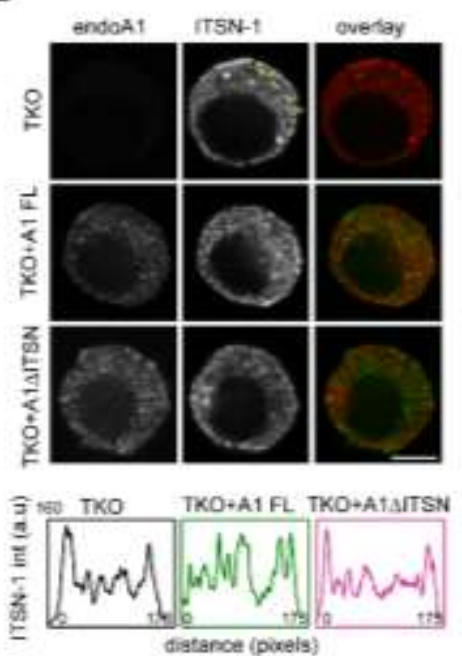

$\mathbf{F}$

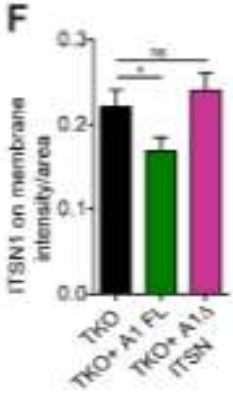

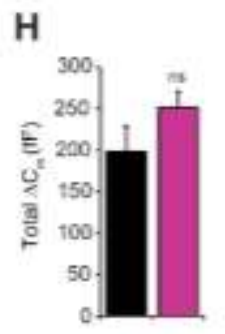
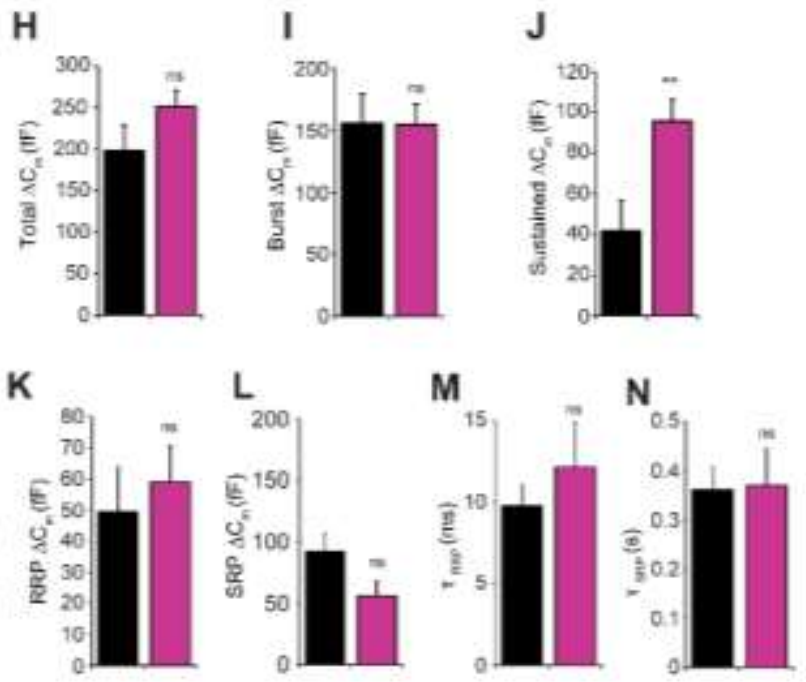

Figure 8. Endophilin's role in exocytosis is mediated, at least in part, through its interaction with intersectin 
(A-B) Distribution of intersectin (ITSN) 1 was altered in the endophilin TKO cells. (C-D) Distribution of ITSN2 was altered in the endophilin TKO cells. Representative confocal images of chromaffin cells stained against ITSN-1 (A) and ITSN-2 (C) at resting and stimulated (depolarization by high $\mathrm{K}^{+}$) conditions. Intensity line profiles below indicate ITSN 1 and ITSN-2 intensities along the line (the approx. line position is marked in yellow in A, scale bar: $3 \mu \mathrm{m}$ ). Quantification of ITSN-1 (B) and ITSN-2 (D) intensities in the cytosol vs. near the membrane (see Methods and Suppl Fig S6) revealed the altered distribution of ITSN-1 and ITSN2 in endophilin TKO cells that did not further change upon stimulation (three independent experiments, p-value<0.0001, one-way ANOVA - multiple comparisons was done by Tukey's multiple comparison test; note that WT resting vs. WT high $\mathrm{K}^{+}, \mathrm{WT}$ resting vs. TKO resting and TKO resting vs. TKO high $\mathrm{K}^{+}$were compared). (E-F) ITSN-1 altered distribution in endophilin TKO cells was rescued by expression of endophilin 1, but not by expression of endophilin 1- $\triangle$ ITSN (endophilin E329K+S366K mutant that does not bind ITSN-1). (G-N) Expression of endophilin 1 in endophilin TKO cells rescued exocytosis as inspected by combined capacitance and amperometry measurements (see Figure 3), but the same effect could not be achieved by expressing endophilin mutant that does not bind ITSN1 (E329K+S366K).

\section{STAR METHODS}

\section{CONTACT FOR REAGENT AND RESOURCE SHARING}

Further information and requests for resources and reagents should be directed to and will be fulfilled by the Lead Contact, Ira Milosevic, imilose@gwdg.de.

\section{EXPERIMENTAL MODEL}

All animal-related procedures were performed according to the European guidelines for animal welfare (2010/63/EU), with the explicit permission from the Niedersächsisches Landesamt für Verbraucherschutz und Lebensmittelsicherheit (LAVES), registration 14/1701. Animals were housed and bred in the Zentrale Tierexperimentelle Einrichtung (ZTE) Göttingen with ad libitum access to water and food. Mice were kept in groups of 1-5 animals on a $12 \mathrm{~h}$ light/12h dark cycle in the individually ventilated cages. Endophilin mutant mice were originally generated and described in (Milosevic et al., 2011), and are accessible from the Jackson Laboratory (strain 021573 - B6;129-Sh3gl2tm1Pdc/J; strain 021574 - B6;129-Sh3gl1tm1Pdc/J; 021575 - B6;129-

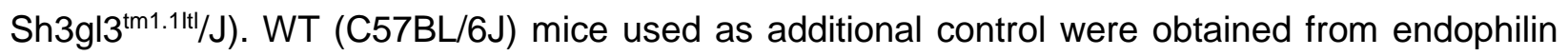
$\mathrm{A} 1^{+/-} \mathrm{A} 2^{+/-} \mathrm{A} 3^{+/-}$mice breeding, or were purchased from ZTE. Both male and female P0 pups were used to prepare the culture of adrenal chromaffin cells as follows: Adrenal glands were isolated from P0 mice (endophilin TKO, endophilin KOWTKO or WT-C57/BL6J) and placed in Locke's solution (154 mM NaCl, $5.6 \mathrm{mM} \mathrm{KCl}, 0.85 \mathrm{mM} \mathrm{NaH}_{2} \mathrm{PO}_{4}, 2.15 \mathrm{mM} \mathrm{Na}_{2} \mathrm{HPO}_{4}$, and $10 \mathrm{mM}$ glucose, $\mathrm{pH} 7.25)$, and cleaned to remove connective tissue. The glands were digested in $200 \mu$ l of enzyme 
solution at $37^{\circ} \mathrm{C}$ for $30-35$ minutes (enzyme solution: DMEM medium with high glucose (Gibco)

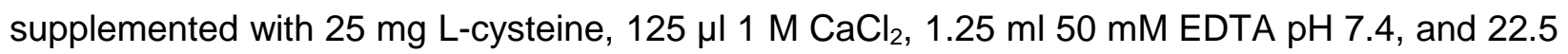
units $/ \mathrm{ml}$ papain). The enzyme was inactivated by adding $200 \mu \mathrm{L}$ inactivating solution for 5 minutes at $37^{\circ} \mathrm{C}$ (inactivating solution: $112.5 \mathrm{ml}$ DMEM medium with high glucose supplemented with 12.5 $\mathrm{ml}$ heat-inactivated fetal calf serum (Life Technologies), $312.5 \mathrm{mg}$ albumin fraction-V, $312.5 \mathrm{mg}$ trypsin inhibitor). This solution was replaced by $180 \mu \mathrm{L}$ enriched DMEM medium and the glands were triturated (5-6 times) using a $200 \mu \mathrm{L}$ pipette (enriched DMEM medium: $6.7 \mathrm{~g}$ DMEM powder with high glucose (Gibco) was dissolved in $496.5 \mathrm{ml}$ double-distilled water supplemented with $0.55 \mathrm{~g} \mathrm{NaHCO}_{3}, 1 \mathrm{ml}$ penicillin/streptomycin (Life Technologies) and $2.5 \mathrm{ml}$ insulin-transferrinselenium-X (Thermo Fischer Scientific). The cells were plated on cleaned and sterilized glass coverslips and allowed to settle for 30-45 minutes before adding $1 \mathrm{~mL}$ enriched DMEM medium. The cells were maintained at $37{ }^{\circ} \mathrm{C}$ and $8 \% \mathrm{CO}_{2}$ and used within $3-4$ days for the experiments.

\section{METHOD DETAILS}

\section{Cloning and virus production}

For the rescue experiments, full length endophilin 1, and endophilin 2, were cloned into lentivirus (LV) expression vector FUGW (a gift of Oliver Schlüter, European Neuroscience Institute Göttingen, Germany) containing an IRES followed by an enhanced green fluorescent protein (EGFP) to allow simultaneous yet independent expression of both proteins. Endophilin 1, and endophilin 2, were first amplified by a PCR reaction (original plasmids were described in Milosevic et al., 2011) and then inserted into the FUGW vector using Xbal and BamHI restriction enzymes. Similarly, endophilin 1-BAR and endophilin 2-BAR constructs (BAR domain and the linker sequence) were cloned by amplifying and inserting the endophilin 1-BAR and 2-BAR sequences into FUGW vector using $\mathrm{Xbal}$ and $\mathrm{BamHI}$ restriction enzymes. Endophilin 1- $\triangle$ ITSN (endophilin 1 $\mathrm{E} 329 \mathrm{~K}+\mathrm{S} 366 \mathrm{~K}$ - mutant that cannot bind intersectin-1; Pechstein et al, 2015) was first generated by QuikChange II Site-Directed Mutagenesis Kit (Agilent) and subsequently inserted into the FUGW vector using $\mathrm{Xbal}$ and $\mathrm{BamHl}$ restriction enzymes. All constructs were verified by sequencing and control restriction digestion. Lentiviral particles were generated as follows: 293FT cells were plated at $1 \times 10^{7}$ per $\varnothing 10 \mathrm{~cm}$ dish. The cells were transfected with lentivirus transfer plasmid as detailed above ( $3^{\text {rd }}$ generation lentivirus system) along with envelop and packaging plasmids using Lipofectamine-2000 and following the manufacturer's protocol (Invitrogen). The 
cells were maintained in the $\mathrm{S} 2$ bio-safety laboratory henceforth and the medium was exchanged $14 \mathrm{~h}$ post-transfection. The medium containing lentivirus suspension was collected, centrifuged at 3,000 RPM for 15 minutes at $4^{\circ} \mathrm{C}$ to remove cell debris. Further, virus was concentrated using Amicon (100K, UFC910096) at 4,000 RPM for 20 minutes at $4^{\circ} \mathrm{C}$. The concentrated particles were diluted up to $2 \mathrm{~mL}$ of Tris-buffer saline (TBS; $\mathrm{pH} 7.4$ ); aliquots were frozen in cryo-tubes in liquid nitrogen and stored in $-80^{\circ} \mathrm{C}$ until being used. The efficiency of the lentivirus was tested by Western blot and imaging of a fluorescent reporter. The virus particles were added 6-8 hours after cell plating, and the cells were used 60-72 hours post infection.

\section{Purification of synaptic vesicles and LDCVs}

Synaptic vesicles were purified as recently published (Farsi et al., 2018). LDCVs were purified according to Park et al. (2012) with minor modifications. Specifically, the medulla was isolated from two bovine adrenal glands obtained from a recently slaughtered animal, was minced in 300 mM sucrose buffer (300 mM sucrose, 20 mM HEPES, pH 7.35, $200 \mu \mathrm{M}$ PMSF) and then homogenized using a cooled teflon-glass homogeniser (Dounce homogenizer, Wheaton) at 1,000 rpm. After centrifugation at $1,000 \mathrm{~g}$ for $15 \mathrm{~min}$ at $4^{\circ} \mathrm{C}$, the pellet was discarded and the supernatant was subjected to another centrifugation at $12,000 \mathrm{~g}, 15 \mathrm{~min}, 4^{\circ} \mathrm{C}$. This step was repeated once more, and the resulting pellet was resuspended in $300 \mathrm{mM}$ sucrose buffer and loaded on top of a continuous sucrose gradient (from $300 \mathrm{mM}$ to $2.0 \mathrm{M}$ ) to remove contaminants. LDCVs were collected from the pellet after centrifugation at $27,000 \mathrm{rpm}$ for $60 \mathrm{~min}$ (Beckman SW 41 Ti rotor) and re-suspended in $120 \mathrm{mM} \mathrm{K}$-glutamate, $20 \mathrm{mM}$ K-acetate, $20 \mathrm{mM} \mathrm{HEPES}, \mathrm{pH}$ 7.4. Protein concentration of SV and CCV samples was determined using Pierce BCA Protein Assay Kit.

\section{Electrophysiological measurements}

The mouse chromaffin cells were maintained in extracellular solution $(145 \mathrm{mM} \mathrm{NaCl}, 2.8 \mathrm{mM} \mathrm{KCl}$, $2 \mathrm{mM} \mathrm{CaCl}_{2}, 1 \mathrm{mM} \mathrm{MgCl}_{2}, 10 \mathrm{mM} \mathrm{HEPES}$, and $2 \mathrm{mg} / \mathrm{ml}$ D-glucose, $\mathrm{pH}$ 7.2, $305 \mathrm{mOsM} / \mathrm{kg}$ ) during the electrophysiological recordings. Capacitance and amperometric measurements were performed in parallel on a Zeiss Observer.D1 equipped with Polychrome V monochromator (Till Photonics), an EPC-10 amplifier (HEKA Elektronik) for patch-clamp capacitance measurements and an EPC-9 (HEKA Elektronik) for amperometry. Catecholamine release was triggered by UVflash photolysis (using a JML-C2, Rapp Optoelektronik) of a caged calcium compound, 
nitrophenyl-EGTA, which was transferred into the cell through a patch pipette. The intracellular calcium was monitored, after the setup calibration was done by infusion of 8 solutions of known calcium concentrations into chromaffin cells, by ratiometric measurement of two calcium dyes with different calcium binding affinity, Fura4F and Furaptra (ThermoFischer Scientific). The excitation light (Polychrome V) was alternated between $350 \mathrm{~nm}$ and $380 \mathrm{~nm}$ to perform the

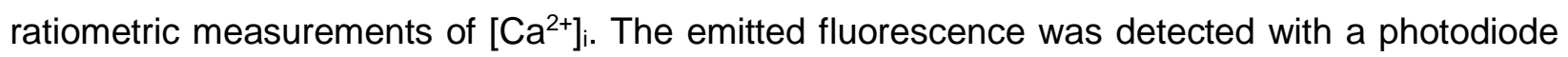
and sampled using Pulse software (HEKA). The same software was used to control the voltage in the pipette and perform capacitance measurements. The intracellular solution contained (in $\mathrm{mM}$ ): $100 \mathrm{Cs}$-glutamate, $8 \mathrm{NaCl}, 4 \mathrm{CaCl}_{2}$, $32 \mathrm{Cs}$-HEPES, $2 \mathrm{Mg}$-ATP, $0.3 \mathrm{GTP}, 5 \mathrm{NPE}, 0.4$ Fura4F and 0.4 Furaptra (L-ascorbic acid was added to prevent flash-induced damage to Fura dyes), $\mathrm{pH}$ 7.2 (osmolarity adjusted to $\sim 295 \mathrm{mOsm}$ ). Amperometric recordings were done using $\varnothing 5 \mu \mathrm{m}$ carbon fibers (Thornel P-650/42, Cytec) insulated by the polyethylene method (Bruns, 2004) and EPC-7 (HEKA). Cells were perfused with intracellular solution for single amperometry spikes, consisting of $70 \mathrm{mM}$ Cs-glutamate, $8 \mathrm{mM} \mathrm{NaCl}, 4 \mathrm{mM} \mathrm{CaCl}$, $22.5 \mathrm{mM}$ Cs-HEPES, $2 \mathrm{mM} \mathrm{Mg}$ ATP, $0.3 \mathrm{mM} \mathrm{Na-GTP,} 37 \mathrm{mM} \mathrm{Ca2}{ }^{+}$DPTA, $0.32 \mathrm{mM}$ Fura-4F and $0.48 \mathrm{mM}$ Furaptra pH 7.2 (osmolarity adjusted to $\sim 300 \mathrm{mOsm}$ ). Fibers were clamped to $700 \mathrm{mV}$. Currents were acquired at $25 \mathrm{kHz}$ and filtered off-line using a Gaussian filter with a cutoff set at $1 \mathrm{kHz}$.

\section{Immunocytochemistry}

Chromaffin cells were cultured for up to $72 \mathrm{~h}$ on poly-L-lysine coated coverslips in a 12-well plate (Sarstedt) for immunocytochemistry experiments. The cells were fixed in freshly prepared $4 \%$ paraformaldehyde (PFA) in phosphate buffered saline (PBS; Sigma) and neutralized afterwards for 20 minutes with $50 \mathrm{mM} \mathrm{NH}_{4} \mathrm{Cl}$ in PBS. Blocking was performed with the blocking buffer containing 3\% bovine serum albumin (BSA; Sigma), $0.2 \%$ cold-fish gelantine (Sigma) and $1 \%$ goat serum (Gibco) for 1 hour, and cells were permeabilized in $0.1 \%$ Triton X-100 (Sigma) in the blocking buffer for 10 minutes. After a brief washing step, the cells were stained with primary antibody overnight at $4^{\circ} \mathrm{C}$ followed by washing and secondary antibody staining in the dark at room temperature for $1 \mathrm{~h}$ (the antibodies are listed in Key Resources Table). The washing procedure was repeated following 2 min incubation with 4',6-diamidino-2-phenylindole (DAPI 1:5,000 in PBS; Sigma) to stain the nucleus of the chromaffin cells. Finally, the coverslips were mounted in Mowiol 4-88 ${ }^{\circledR}$ mounting medium (Sigma). Images were acquired using Zeiss LSM 710 laser scanning 
confocal microscope or Zeiss LSM 800 Airyscan confocal microscope (63x objective, numerical aperture 1.4).

For chromaffin cell stimulation, the cells were washed carefully in pre-warmed Locke's solution before incubation in extracellular (control condition) or high $\mathrm{K}^{+}$solution $(88 \mathrm{mM} \mathrm{NaCl}, 59 \mathrm{mM} \mathrm{KCl}$, $2 \mathrm{mM} \mathrm{CaCl}_{2}, 1 \mathrm{mM} \mathrm{MgCl}$, $10 \mathrm{mM} \mathrm{HEPES}$, and $2 \mathrm{mg} / \mathrm{ml}$ D-glucose, $\mathrm{pH}$ 7.20, $300 \mathrm{mOsM} / \mathrm{kg}$ ) for 3 minutes at RT. The cells were placed on ice immediately, fixed in 4\% PFA (freshly prepared) for 10 minutes on ice followed by 20 minutes at RT. Immunostainings and image acquisition were performed as described above.

\section{Electron microscopy on adrenal chromaffin cells}

Adrenal glands from endophilin TKO, endophilin KOWTKO and WT PO mice were isolated and subsequently fixed in 4\% PFA + 0,5\% glutaraldehyde (GA; Sigma) in $0.1 \mathrm{M} \mathrm{PBS,} \mathrm{pH} 7.2$ for 1 hour on ice, and afterwards in $2 \% \mathrm{GA}$ in $0.1 \mathrm{M}$ sodium cacodylate buffer ( $\mathrm{pH} 7.2$; Sigma) overnight at $4^{\circ} \mathrm{C}$. The next day, the adrenal glands were washed 3 times for 10 minutes in $0.1 \mathrm{M}$ sodium cacodylate buffer. Post-fixation was done on ice for 1 hour in $1 \%(\mathrm{v} / \mathrm{v}) \mathrm{OsO}_{4}$ in cacodylate buffer, followed by further washing steps (2x10 min cacodylate buffer, $3 \times 5$ min water). En-bloc staining of adrenal glands was performed using $1 \%(\mathrm{v} / \mathrm{v})$ uranyl acetate (Sigma) in water for $1 \mathrm{~h}$ on ice. Subsequent to three brief washing steps in water, adrenal glands were dehydrated in an ascending ethanol series (5 min 30\%, 5 min 50\%, 10 min 70\%, 2x10 min 95\%, 3×12 min 99,9\% ethanol) and infiltrated in Epon resin (50\% ethanol + 50\% epon for $30 \mathrm{~min}$ and for $90 \mathrm{~min}, 100 \%$ epon for $\sim 20 \mathrm{~h}$ ) at room temperature. The samples were placed in the embedding molds and polymerized for $48 \mathrm{~h}$ at $70^{\circ} \mathrm{C}$. Ultrathin sections $(65 \mathrm{~nm})$ were cut using a Leica UC6 ultra microtome, placed on formvar-coated copper grids, and stained in uranyl acetate and lead citrate (using the Reynold's method). Images were acquired using a JEOL JEM-1011 transmission electron microscope equipped with a Gatan Orius 1200A camera at 6000x magnification, on average 5 images/cell were acquired.

\section{Endocytosis assays on chromaffin cells}

Transferrin (Tf) was conjugated to Alexa Fluor ${ }^{\mathrm{TM}} 546$ (5 $\mu \mathrm{g} / \mathrm{mL}$; Invitrogen, Cat.\# T23364), and the uptake assay in adrenal chromaffin cells was performed as described in Chen et al. (1998). All images were captured under the same acquisition settings using a Zeiss 810 Airyscan confocal 
microscope. Analyses of Tf-A546 data (z-stack of the whole cells) was performed by Imaris (Bitplane) using the Spot module and statistics was performed by one-way ANOVA. Non-toxic recombinant cholera toxin subunit-B (CT-B) was conjugated to Alexa Fluor ${ }^{\mathrm{TM}} 594$ (Thermo Fischer, Cat.\# C22842) and the uptake assay was adapted to adrenal chomaffin cells using the protocol from Kirkham et al. (2005). In short, uptake of $2 \mu \mathrm{g} / \mathrm{mL}$ CT-B-A594 was carried out at $37^{\circ} \mathrm{C}$ in serum-free medium (Gibco) for $8 \mathrm{~min}$ (note that CT-B-A594 attaches to cells by binding to ganglioside $\mathrm{GM}_{1}$ ), Cells were washed $4 \times 30$ s with the extracellular buffer to remove CT-B-A594 cell surface labeling, fixed in 4\% PFA for $20 \mathrm{~min}$, and imaged with a Zeiss 810 confocal microscope. Images were captured through the equatorial plane of each cell (one plane only) using the same acquisition settings, and were analyzed in ImageJ (a background-threshold was applied and every fluorescent cluster greater than six pixels was counted).

Live imaging of chromaffin cells labeled with mCling-Atto647N (Synaptic Systems) was done using the spinning disk confocal microscope with temperature control unit (kept at $37 \mathrm{C}$ ) and custom-built imaging chamber. Cells were maintained in extracellular solution in imaging chamber and stained with $0.5 \mathrm{nmol} / \mathrm{ml} \mathrm{mCling}$-Atto647N for 1 minute. The solution was exchanged and cells were washed rapidly (few seconds) before image acquisition. Images were captured up to 8 minutes after addition of $\mathrm{mCling}$-Atto647N through the middle of each cell (using the same acquisition settings), and quantified using ImageJ as detailed above for CT-B-A594. Statistics was performed by one-way ANOVA.

\section{Quantification and statistical analysis}

Endophilin-CgA colocalization analysis in chromaffin cells was performed as follows: colocalization was evaluated using object-based overlap and JACoP plugin in ImageJ since endophilin signals had a cytosolic component. Specifically, a region-of-interest (ROI) was chosen so it did not contain nucleus, and the images were segmented into objects and background (bright fluorescent objects were segmented from the image) before Pearson's correlation coefficient was calculated. The same analysis was performed with the $90^{\circ}$ rotated image, and this random value was subtracted for each cell. In addition, the complementary manual colocalization analysis was performed: here, circles were superimposed on bright fluorescent spots in the $\mathrm{CgA}$ channel and transferred to identical image locations in the endophilin channel. If the fluorescence intensity maximum in the endophilin channel was located in the same circle and the morphology of the 
signal resembled that of the $\mathrm{CgA}$ signal, the circle was rated as positive (colocalized). If this was not a case, it was rated as negative (not colocalized). To correct for random colocalization of two abundant signals, circles were also transferred to a $90^{\circ}$ rotated image of the endophilin channel. A minimum of 10 images from 3-5 experiments were analyzed for each genotype/condition. Two approaches (semi-automatic ImageJ-based and manual) gave similar result.

The kinetic analysis of the capacitance measurements was performed by fitting individual capacitance traces with a triple-exponential function using a custom-written macro in IGOR Pro, as described in Milosevic et al., 2005. The amplitudes and time constants of the two faster exponentials define the size and release kinetics of the slowly releasable pool (SRP) and the readily releasable pool (RRP), respectively. Filtering, spike detection and analysis of aperometric spikes were performed by a custom-written macro (Mosharov and Sulzer, 2005) in IGOR Pro (Wave Metrics). Data are represented as mean \pm SEM, and unpaired t-test or nonparametric Kruskal-Wallis test with Dunn's multiple comparison test were used to test statistical difference, which is indicated by ${ }^{*} p<0.05,{ }^{* *} p<0.01$, and ${ }^{* * *} p<0.001$.

The ultrastructural analysis by EM: At least 23 cells from 4 different animals and independent embeddings per group were analyzed using the IMOD (bio3d.colorado.edu/imod) and ImageJ software. The area of chromaffin cells was defined as area within the plasma membrane, thereby excluding the nucleus area. The area of LDCVs was directly measured using the area selection tool in ImageJ, and distances were measured between LDCV membrane and plasma membrane in IMOD. The statistics is done by one-way ANOVA (Figure 4B-D) followed by Tukey's post-hoc test (Figure 4E) and Kolmogorov-Smirnov test (Figure 4F-F'; to ensure that the detected differences were not artefacts created by multiple comparisons, we applied Bonferronicorrection).

For quantification of $\mathrm{CgA}$-positive vesicles (Figure $5 \mathrm{~A}-\mathrm{B}$ ) and Syt-1 intensity on $\mathrm{CgA}$-positive vesicles (Figure $5 \mathrm{C}$ and $5 \mathrm{E}$ ), three-dimensional surface reconstruction was carried out with the "Cell with organelles" module of Imaris software, version 8.0.2 (Bitplane).

For quantification of intensity of proteins detected by immunofluorescence (Figure 5D, Figure G and Figure 6A), the mean fluorescence intensity was measured in the cell cytoplasm, excluding the nucleus, using ImageJ and normalized to the measured area. The data are represented as mean \pm SEM and statistics was performed by unpaired t-test. 
To characterize the distribution of intersectins (Figure 8A-F; Suppl Fig S6D-G), we capitalized on the round-shape of adrenal chromaffin cells, and defined a ROI-based analysis approach in Image J software. We defined two concentric ROls: the outer circular ROI around the whole cell and the inner circular ROI (to measure the intensity of the cytosol). The inner circular ROI was defined to be 40 pixels less than the radius of the outer ROI. The intensities of two ROls were then measured, and the inner circular ROI was subtracted from the outer circular ROI to calculate the near-membrane intensity. The ratio of intensities between membrane and cytosol was plotted. The data are represented as mean \pm SEM from three independnet experiemnts with at least 45 cells per group and the statistics was performed by one-way ANOVA.

\section{SUPPLEMENTAL INFORMATION}

\section{Supplemental Material and Methods}

Adrenal chromaffin cells on poly-L-lysine-coated glass coverslips were washed carefully in prewarmed Locke's solution before incubation in extracellular (control condition) or high $\mathrm{K}^{+}$solution (88 mM NaCl, $59 \mathrm{mM} \mathrm{KCl}, 2 \mathrm{mM} \mathrm{CaCl}, 1 \mathrm{mM} \mathrm{MgCl}, 10 \mathrm{mM} \mathrm{HEPES}$, and $2 \mathrm{mg} / \mathrm{ml} \mathrm{D-glucose,}$ $\mathrm{pH} 7.20,300 \mathrm{mOsM} / \mathrm{kg}$ ) for 3 minutes at RT. The cells were then fixed in freshly prepared $4 \%$ PFA for 30 minutes at RT and neutralized with $50 \mathrm{mM} \mathrm{NH}_{4} \mathrm{Cl}$ in PBS for 20 minutes. The cells were then permeabilized and blocked for unspecific binding using blocking solution (3\% BSA, $0.2 \%$ coldfish gelantine, $1 \%$ goat serum, $0.1 \%$ Triton $X-100$ in PBS, pH 7.4) at RT. Subsequently, the cells were incubated for $30 \mathrm{~min}$ with phalloidin-Texas Red dye for staining F-actin (1U/cs; $200 \mathrm{U} / \mathrm{mL}$ stock dissolved in methanol), washed, stained with DAPI and mounted using Mowiol$488^{\circledR}$ mounting medium. The images were acquired using Zeiss LSM 800 laser scanning confocal microscope (63x objective, numerical aperture 1.4). The actin staining was analyzed by ImageJ PLASMaCC macro reported in Kurps et al. (2014). Briefly, the signal in the proximity of plasma membrane was measured by a polar transformation function in ImageJ and the mean intensity and average density was calculated. The data are represented as mean $\pm S E M$, and statistics was performed by one-way ANOVA.

The band signal intensity in Western blots were quantified by measuring the mean intensity of $\mathrm{ROI}$ in ImageJ software, and subtracting the background signal from the adjacent area. 


\section{Supplemental Figures}

A

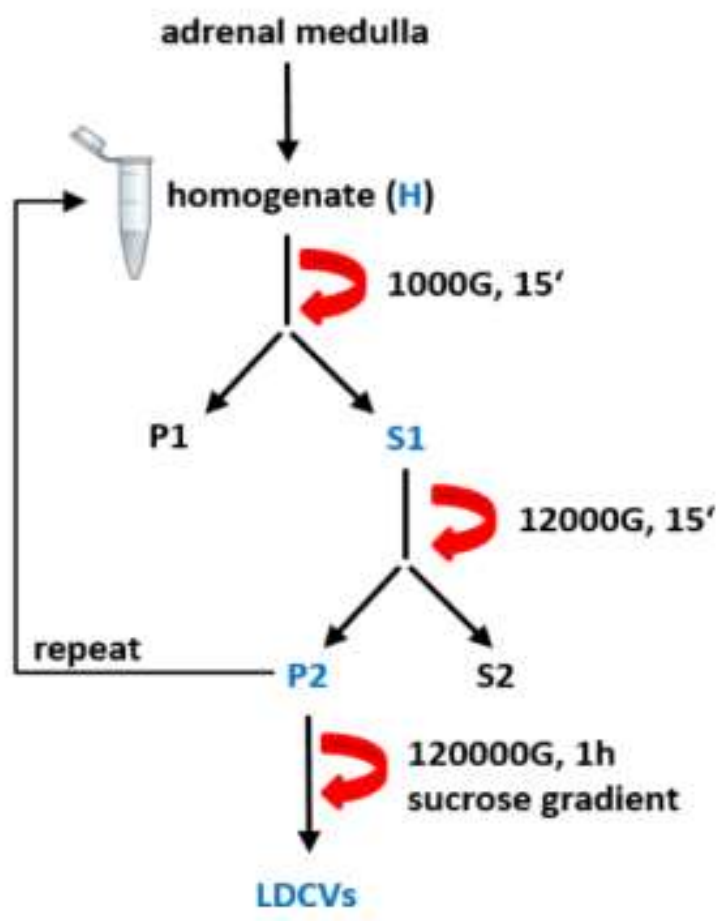

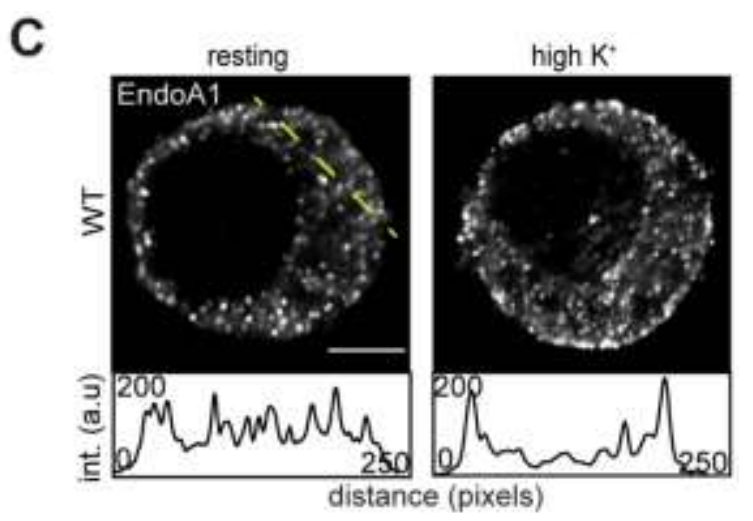
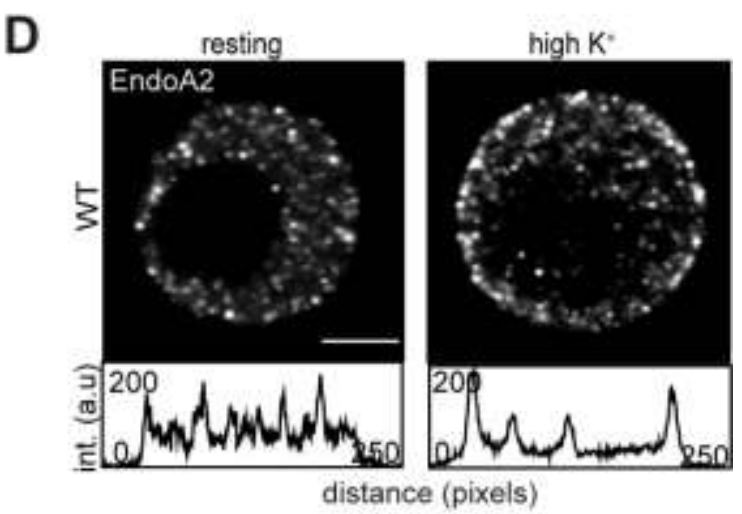

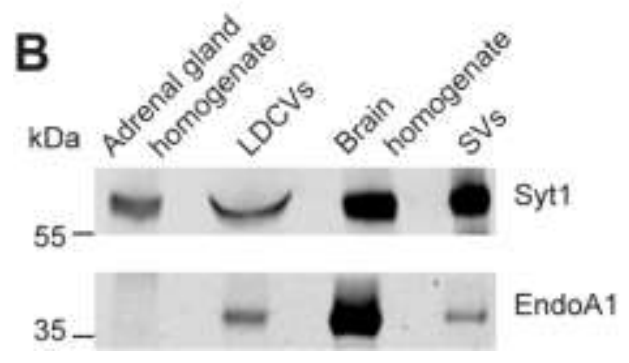

Figure S1 (related to Figure 1). Endophilin on isolated LDCVs and re-distribution of

\section{endophilin upon chromaffin cell stimulation}

(A) Scheme of the protocol used to isolate LDCVs from the medulla of bovine adrenal glands. (B) Immunoblot analysis of LDCVs isolated from bovine adrenal medulla and SVs from mouse brain revealed the presence of endophilin 1 at LDCVs and SVs. Syt1 was used as a vesicle marker protein. Same volume

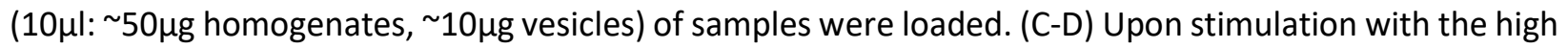
$\mathrm{K}^{+}$solution, distributions of endophilin 1 and endophilin 2 were mildly altered - more endophilin 1 and endophilin 2 were present at the plasma membrane after stimulation, as indicated by the intensity line profiles. Scale bar $3 \mu \mathrm{m}$. 


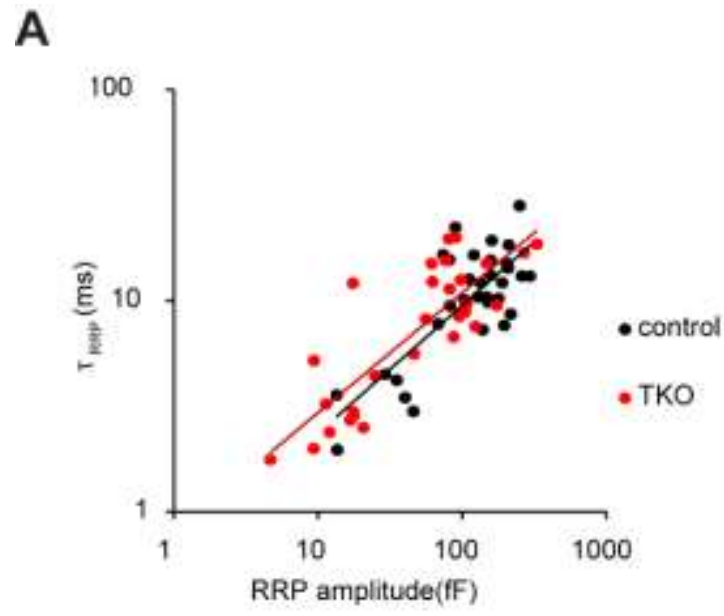

Figure S2 (related to Figure 2). Double-log plot shows correlation between the lower time constant of the RRP and the lower pool size of the RRP

The RRP was smaller (contained fewer vesicles), but RRP vesicles fused faster in the endophilin TKO chromaffin cells (see Figure 2). We considered the possibility that those two phenotypes are linked. Double-log plot of time vs. the pool size of the RRP showed correlation, and suggested that the lower pool size and the faster time constants of fusion may constitute a single phenotype. A similar observation was made in synaptotagmin-7 KO cells (Schonn et al., 2008). 
A

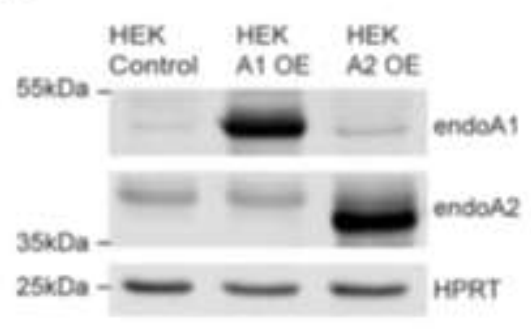

C

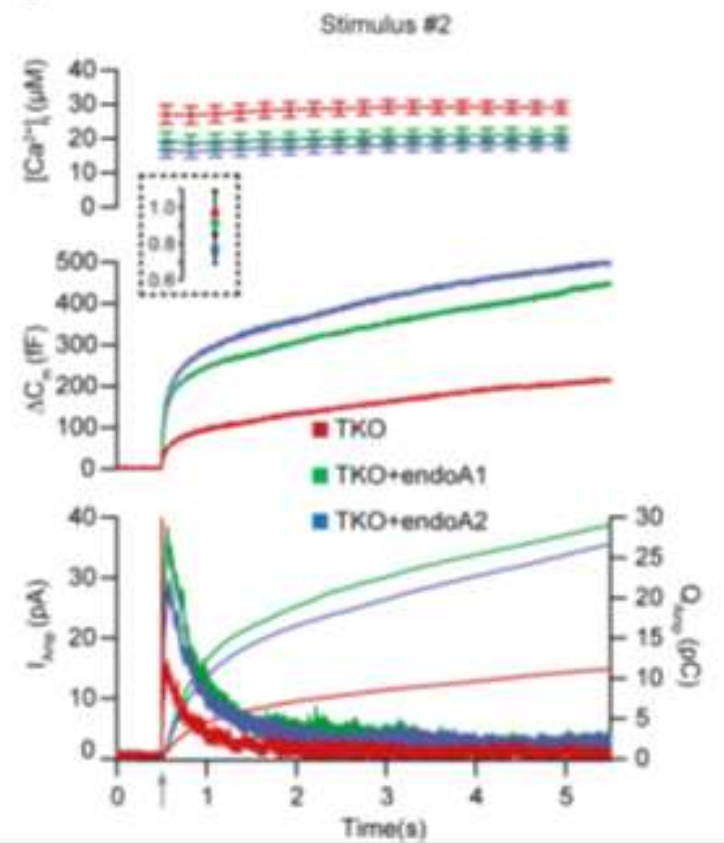

B

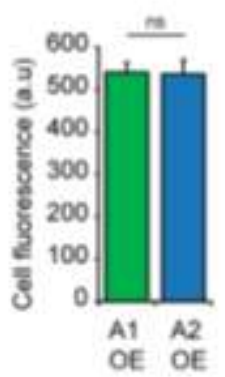

D

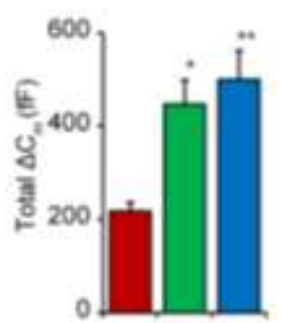

E

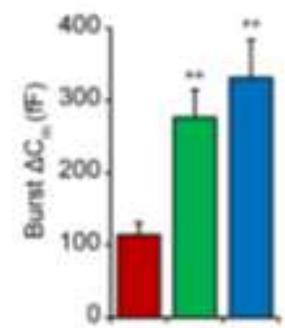

$\mathbf{F}$

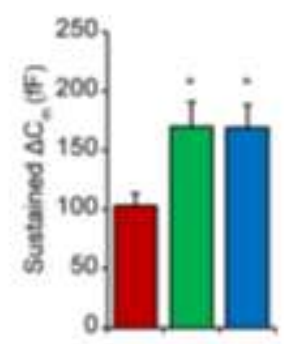

G

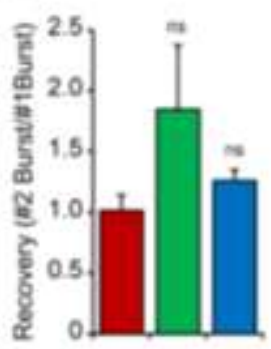

Figure S3 (related to Figure 3). Expression of endophilin 1 and endophilin 2 rescued exocytosis in endophilin TKO cells ( $2^{\text {nd }}$ stimulus)

(A) Verification of lentiviral expression system for endophilin 1 and endophilin 2 in HEK293 cells. Note that the two proteins were expressed to similar levels (quantification is not possible due to the use of two different antibodies). (B) EGFP fluorescence intensities measured from cells expressing endophilin 1 and endophilin 2 using bicistronic lentiviral systems were comparable (50 cells from 3 independent experiments). (C-D) Expression of endophilin 1 and endophilin 2 rescued the exocytic defects seen in endophilin TKO cells (stimulus \#2). Panel arranged as in Figure 3A, with 3 groups: endophilin TKO (red traces; mean of 24 cells), endophilin TKO + endophilin 1 (green traces; mean of 23 cells) and endophilin TKO + endophilin 2 (blue traces; mean of 25 cells). Endophilin KOWTKO data from Figure 2 (black trace; mean of 30 cells) were superimposed. Note that endophilin 1 as well as endophilin 2 could rescue exocytosis, but endophilin 2 was more efficient. (E-F) Burst and sustained component were rescued upon expression of endophilin 1 and 2, respectively. (G) Measure of recovery, calculated as the ratio of burst secretion of the second over the first stimulus was not significantly altered. 
A

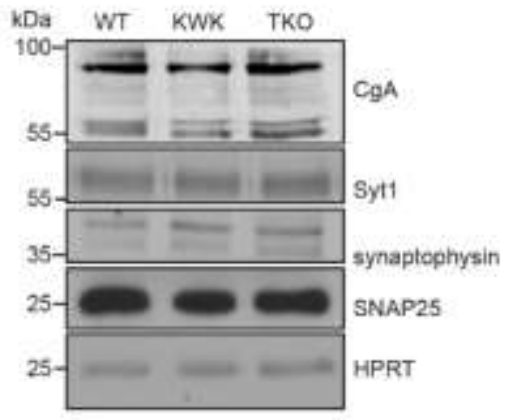

B
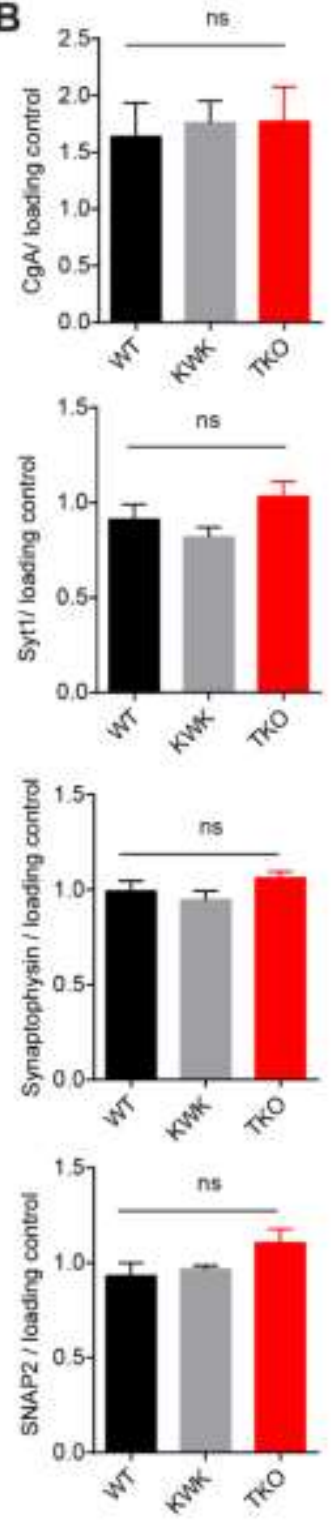

C

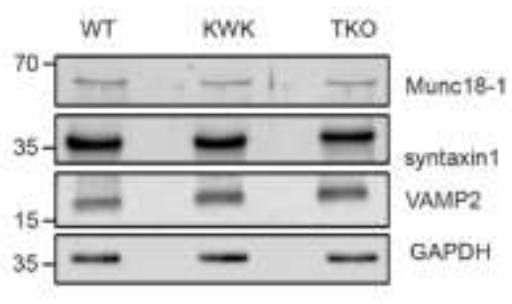

Figure S4 (related to Figure 5).

Levels of key exocytic

proteins were unaltered in mouse adrenal gland homogenates

(A) Representative Western blots for chromogranin-A (CgA), synaptotagmin-1

(Syt-1), synaptophysin and SNAP25 showed no difference between total protein levels in endophilin TKO cells and controls (littermate KOWTKO and WT). Quantification of Western blot data shown in A (one-way ANOVA, 3-4 independent experiments were performed). (C-D) Western blots for Munc18-1, syntaxin-1 and VAMP2 showed no difference between total protein levels in endophilin TKO cells and controls (littermate KOWTKO and WT). Quantified in (D) (one-way ANOVA, at least three independent experiments were performed). 
A

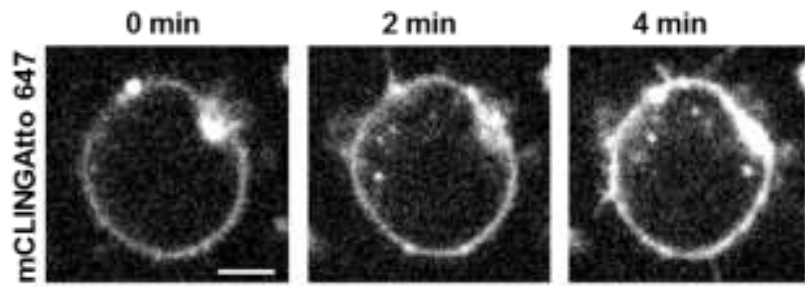

B
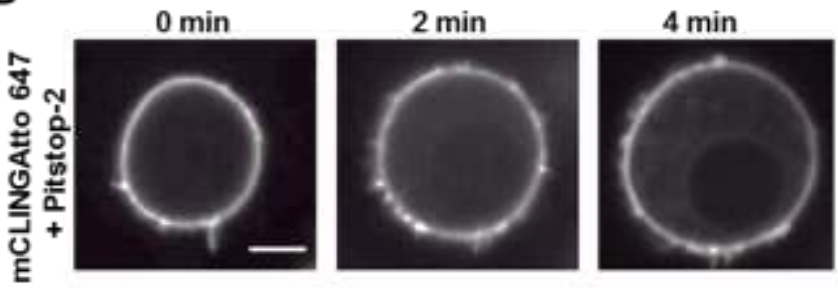

C
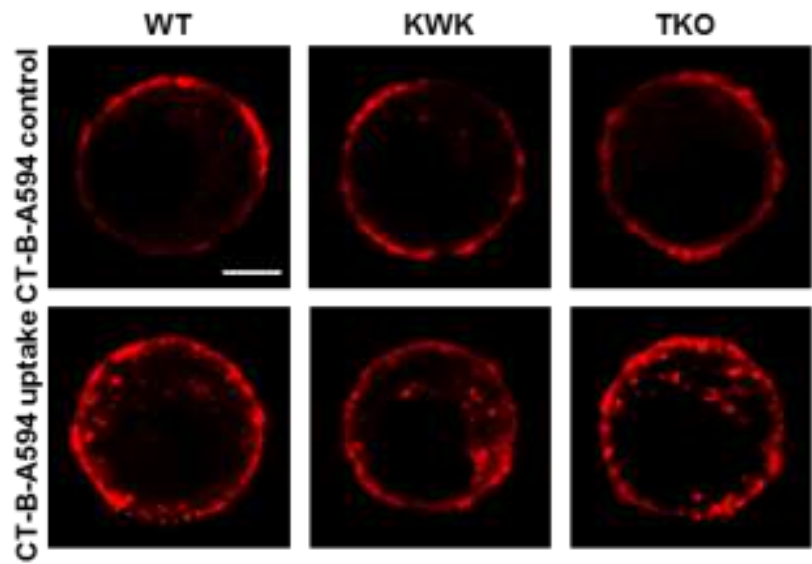

D

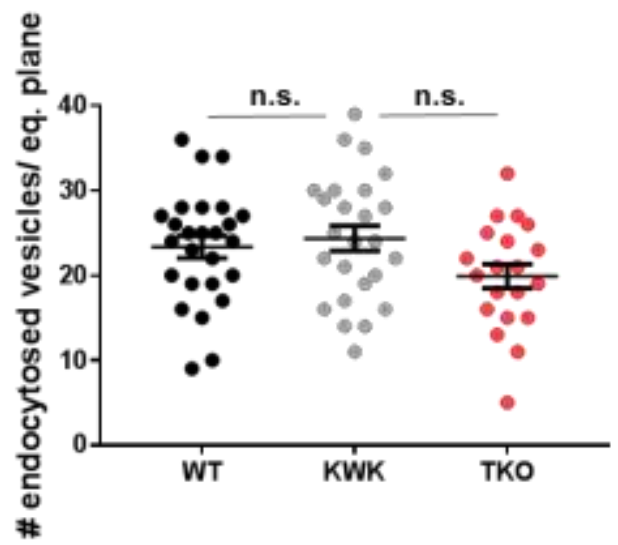

Figure S5 (related to Figure 6). Characterization of uptake of mCling-Atto647 and cholera toxin-B-A594 endocyctic assays in adrenal chromaffin cells

(A) WT mouse chromaffin cells were incubated with either negative control for Pitstop-2 (A) or Pitstop-2 (B) for $10 \mathrm{~min}$ prior the start of the experiment. Images were acquired at 30s, $2.30 \mathrm{~min}$ and $4.30 \mathrm{~min}$ after mCling-Atto647 dye addition, stimulation (high $\mathrm{K}^{+}$) was applied $30 \mathrm{~s}$ later (shown as 0, 2 and $4 \mathrm{~min}$ in the panel). Scale bar 2 um. Considerably lower uptake of mCling-Atto647 was observed in the presence of Pitstop-2. (B-C) Endocytic uptake in chomaffin cells was also tested using CT-B-A594 (non-toxic recombinant cholera toxin subunit-B conjugated to Alexa Fluor 594). Scale bar $1.5 \mu \mathrm{m}$. Endophilin TKO cells were compared to the littermate controls (endophilin KOWTKO and WT; note that an uptake in only one equatorial optical plane was analyzed, not in the whole cell as in the case of transferrin). Quanified in (C) (min. 20 cells from 2 independent experiemnts, one-way ANOVA). 


\section{A}

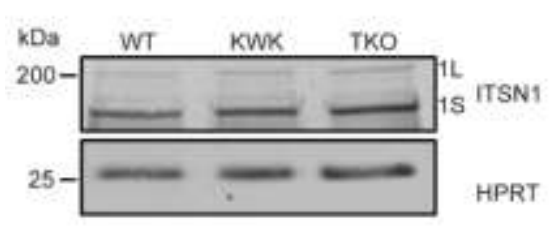

C

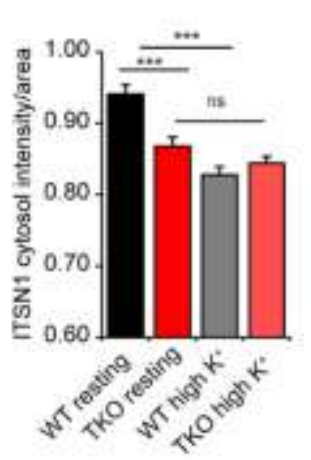

G

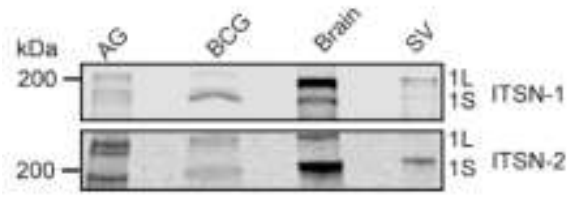

B

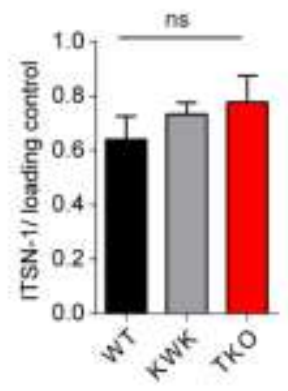

E

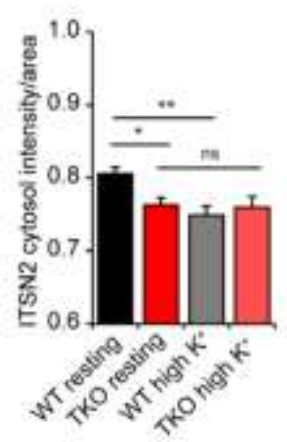

$\mathbf{F}$

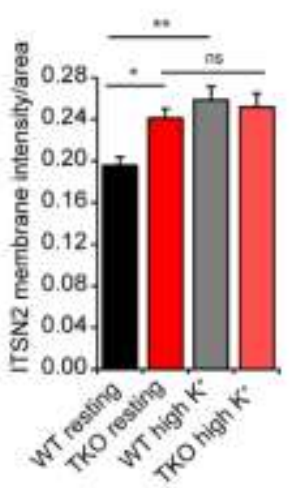

H

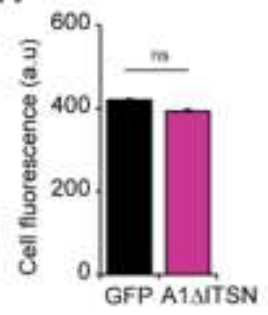

1

Figure S6 (related to Figure 8). Levels of intersectin-1 protein were not altered in endophilin TKO cells

(A-B) Western blot for intersectin 1 (ITSN-1) showed no difference between total ITSN-1 protein levels in endophilin TKO and controls (endophilin KOWTKO and WT); quantified in (B) (one-way ANOVA, three independent experiments). (C) Immunoblot analysis of LDCVs (as in Figure S1B) against ITSN-1 and ITSN2 detected the presence of small amount of ITSN-1 and ITSN-2 proteins at LDCVs and SVs. (D-E) Quantification of ITSN-1 distribution in cell cytosol (D) and near the membrane (E) at resting and stimulated (depolarization by high $\mathrm{K}+$ ) conditions showed altered ITSN-1 distribution in the endophilin TKO cells (mean \pm SEM; one-way ANOVA). (F-G) Quantification of ITSN-2 (same as ITSN-1) on cell cytosol $(F)$ and near the membrane $(G)$ are represented as bar graphs (mean \pm SEM; one-way ANOVA). The ratio of membrane to cytosol intensity is shown in Figure 8A-D. 
A

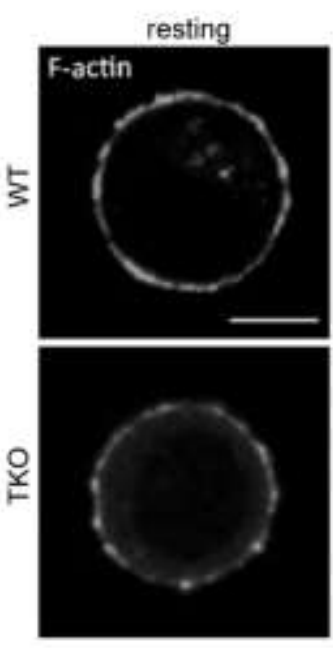

B

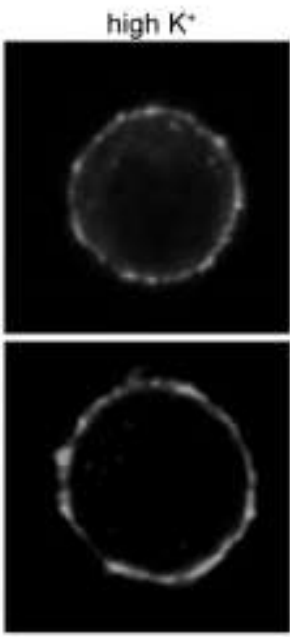

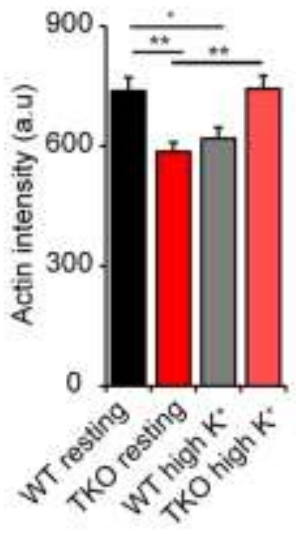

C

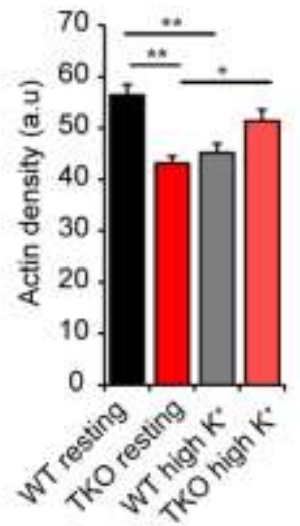

Figure S7 (related to Figure 8). F-actin network is altered in the endophilin TKO cells

(A) Confocal image (through the equatorial plane) of WT and endophilin TKO chromaffin cells stained with phalloidin-Texas Red at resting vs. stimulated (depolarization by high $\mathrm{K}^{+}$solution) conditions. Note that the intensity and density of F-actin was lower in the proximity of the plasma membrane in endophilin TKO cell at resting condition. While stimulation in WT cells led to the depolymerisation of the actin barrier and the re-polymerisation of the de novo actin filaments at the plasma membrane, the opposite was detected in TKO cells. Scale bar $2 \mu \mathrm{m}$. (B-C) Quantification of the cortical F-actin intensity (B) and average density (C) using PLasMACC plugin in ImageJ software (30 cells, one-way ANOVA). 


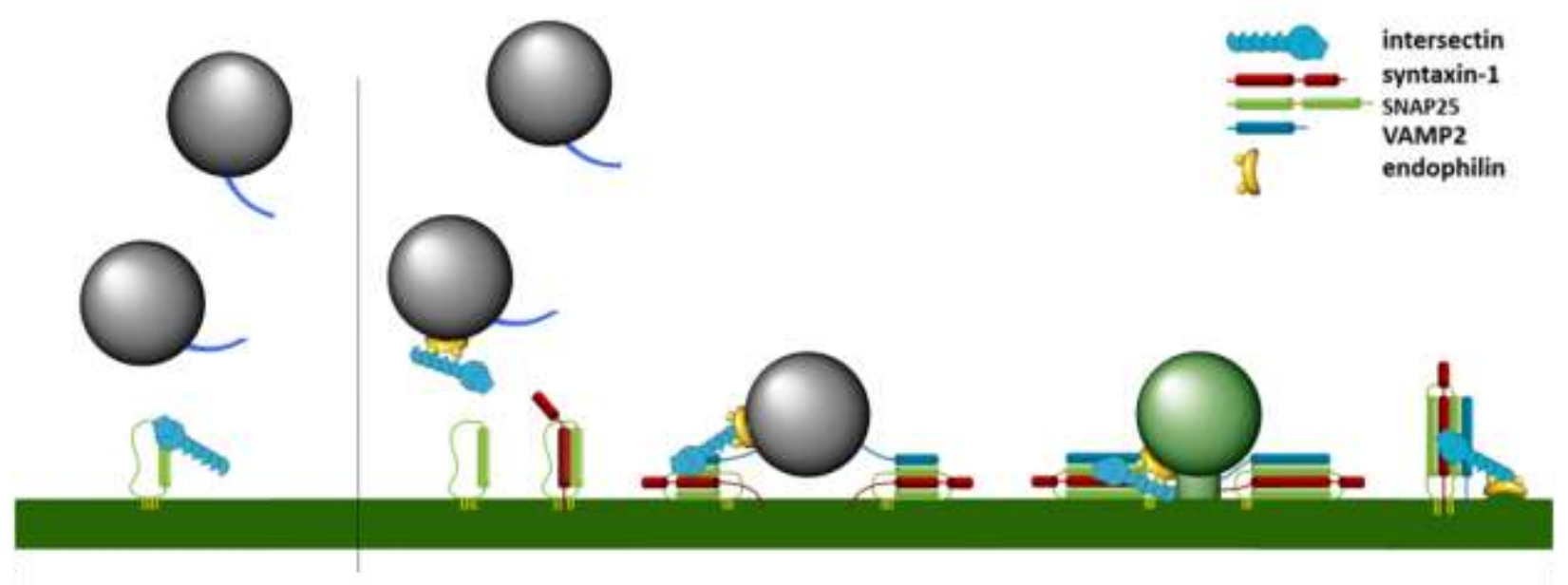

Figure S8. Model of endophilin and intersectin's role in exocytosis

Endophilin is present on at least some neurosecretory vesicles and has a role in the vesicle recruitment, priming and fusion. It can also regulate intersectin localization, possibly to ensure that intersectin acts only at the optimal time. (Left) Without endophilin, intersectin mislocalizes to the plasma membrane so its reported role in exocytosis is likely altered in endophilin TKO cells. (Right) We propose that endophilin and intersectin act in tandem to stimulate the recruitment of secretory vesicles to the plasma membrane and to their site of release, expectedly through modulation of the actin network. In addition, endophilin and intersectin may stabilize the SNARE complex between the vesicular VAMP2/synaptobrevin-2 and plasma membrane-resident SNAP25 and syntaxin-1. We also propose that, once recruited to the plasma membrane, endophilin and intersectin do not dissociate after exocytosis but take part in the subsequent endocytic steps. As such, endophilin and intersectin act as a scaffold that couples exocytic and endocytic events.

In addition to data presented here, this model is supported by our previous results on endophilin and intersectin (Milosevic et al., 2011; Pechstein et al, 2015) and a number of other reports (e.g., Okamoto et al., 1999; Malacombe et al., 2006; Yu et al., 2009; Momboisse et al., 2010; Bai et al., 2010; Winther et al., 2013; Sakaba et al, 2013; Gerth et al., 2017). 


\subsection{Rabconnectin-3a regulates vesicle acidification at the neuronal synapse}

Sindhuja Gowrisankaran ${ }^{1}$, Andrea Raimondi ${ }^{2}$, Zohreh Farsi ${ }^{3}$, Nicolas de Roux ${ }^{4}$, Ira Milosevic ${ }^{1}$

${ }^{1}$ European Neuroscience Institute (ENI), Synaptic Vesicle Dynamics Group; University Medical Center Göttingen (UMG), Göttingen, Germany

${ }^{2}$ Advanced Light and Electron Microscopy Biolmaging Center (ALEMBIC), Hospital San Raffaele, Milano, Italy

${ }^{3}$ Max-Planck Institute for Biophysical Chemistry, Department of Neurobiology, Göttingen, Germany

${ }^{4}$ Inserm U1141, Hospital Robert Debré, University Paris Diderot, Paris, France

- Rbcn-3a is found on SV and CCVs and localizes at the coated pits together with vATPase

- Lack of Rbcn-3a leads to defects in SV acidification and SV recycling, but not endocytosis

- Neurons without Rbcn-3a prominently accumulate lysosome-like structures

- Rbcn-3a promotes stable v-ATPase activity and Rab3 recruitment to SVs 


\section{Rabconnectin-3a regulates vesicle acidification at the neuronal synapse}

Sindhuja Gowrisankaran ${ }^{1}$, Andrea Raimondi ${ }^{2}$, Zohreh Farsi $^{3}$, Nicolas de Roux ${ }^{4}$, Ira Milosevic ${ }^{1 \#}$

${ }^{1}$ European Neuroscience Institute (ENI), Synaptic Vesicle Dynamics Group; University Medical Center Göttingen (UMG), Göttingen, Germany

${ }^{2}$ Advanced Light and Electron Microscopy Biolmaging Center (ALEMBIC), Hospital San Raffaele, Milano, Italy

${ }^{3}$ Max-Planck Institute for Biophysical Chemistry, Department of Neurobiology, Göttingen, Germany

${ }^{4}$ Inserm U1141, Hospital Robert Debré, University Paris Diderot, Paris, France

\#Corresponding author:

Dr Ira Milosevic

E-mail: imilose@gwdg.de

European Neuroscience Institute (ENI)

University Medical Center Göttingen (UMG)

Göttingen, Germany

\section{SUMMARY}

The regulation and timing of vesicle acidification is essential for numerous cellular processes, from lysosomal degradation to refilling of synaptic vesicles (SVs) at the neuronal synapse. Acidification of vesicles is achieved by vacuolar ATPases ( $v$-ATPases), a family of proton pumps that controls the $\mathrm{pH}$ gradient across organelle membranes. Despite their critical importance at the synapse and in many intracellular trafficking routes, the regulation of v-ATPase activity remains poorly understood. We cloned human DMXL2 gene encoding Rabconnectin-3a (Rbcn-3a) whose mutations were reported as a cause of polyendocrine-polyneuropathy syndrome (PEPNS). We found Rbcn-3a to be present on several organelles that acidify, including SVs. Upon deletion of Rbcn-3a, mice show early embryonic lethality. When Rbcn-3a is eliminated from neuronal cells in culture, neurons developed normally, but their activity was perturbed. Neurons without Rbcn-3a failed to fully acidify SVs, showed altered SV recycling and mislocalized Rab3, a key exocytic protein. Curiously, the synapses of neurons without Rbcn-3a accumulated lysosome-like structures and lysosomal marker proteins, suggesting an unanticipated connection between the machineries for endocytosis, acidification and cellular trafficking pathways. 


\section{INTRODUCTION}

Neurotransmitters (NTs) are released by $\mathrm{Ca}^{2+}$-triggered exocytosis of synaptic vesicles and subsequently recycled by endocytosis (Ceccarelli et al., 1973; Heuser and Reese, 1973). New SVs can be derived directly by uncoating of endocytosed clathrin-coated vesicles (Murthy and Stevens, 1998; Ryan et al., 1997; Takei, 1996), or re-formed from the endosome-like structures (Miller and Heuser, 1984; Pfeffer and Kelly, 1985; Watanabe et al., 2014). In either case, newlyformed SVs need to be refilled with neurotransmitter, and this process is driven by a protonelectrochemical gradient established by a vacuolar ATPase ( $v$-ATPase), a family of multi-subunit transmembrane proton pumps that controls the $\mathrm{pH}$ gradient across organelle membranes (Farsi et al., 2017; Hnasko and Edwards, 2012; Jefferies et al., 2008). V-ATPase is comprised of several subunits that form two domains: the cytosolic $\mathrm{V}_{1}$ domain that catalyzes ATP hydrolysis and the membranous $V_{0}$ that translocates protons across membranes. In the $v$-ATPase assembly/disassembly cycle, $\mathrm{V}_{1}$ domain dissociates from $\mathrm{V}_{\mathrm{o}}$ into the cytoplasm and remains as a complex, except the subunit $\mathrm{V}_{1} \mathrm{C}$, which gets released upon disassembly. $\mathrm{V}_{1} \mathrm{C}$ interacts with $\mathrm{V}_{1} \mathrm{E}$ and $V_{1} G$ of $V_{1}$ and a subunit of $V_{0}$ (Inoue et al., 2005), and likely has a regulatory role in proton pump disassembly/reassembly cycle (Beyenbach and Wieczorek, 2006; Bodzęta et al., 2017). vATPase assembly was found to be tightly regulated by vesicle $\mathrm{pH}$ and coupled to exo-endocytosis cycle ina mechanism where the absence of $V_{1}$ domain on fusing $S V_{s}$ was proposed as a likely alternative for favorable fusion of fully filled SVs (Bodzęta et al., 2017). Importantly, the regulation and timing of vesicle acidification is not only essential for the proper function of the synapse, but also for many intracellular trafficking routes and numerous cellular processes, from signaling in endosome to the macromolecule degradation in lysosomes.

How and when a newly (re-)formed vesicle becomes a fusion competent SV is not known. It is proposed that after NT refilling, newly formed SVs can recruit peripheral proteins, primarily Rab3 and synapsin that decide their fate: to undergo the next round of exocytosis or be captured in a cluster/functionally distinct pools (when the recycling process becomes rate limiting, e.g. during prolonged stimulation, SVs are mobilized from clusters to ensure SV availability) (Rizzoli and Betz, 2005). Several molecular players participating in the clustering of SVs have been identified; however, much remains to be understood about the SV clustering in the context of vesicle 
recycling process at the synapse. Rab GTPases and their effectors coordinate vesicle budding, vesicle motility and tethering of vesicle to the target compartment (Zerial and McBride, 2001). Specifically, Rab3 is recruited to the SVs depending on its GTP/GDP binding state and is actively cycled in the synapse (Fischer von Mollard et al., 1990). The Rab3 cycle is regulated by Rab dissociation inhibitor (GDI), GDP/GTP exchange factor (GEF) and GTPase-activating protein (GAP). During or after fusion, Rab3 dissociates from SVs mediated by Rab3-GAP and the dissociated Rab3 in the cytosol remains in association with GDI (Fischer von Mollard et al., 1991). Upon stimulation, Rab3 is activated by exchanging GDP for GTP by Rab3-GEF in association with an unidentified molecule. It is not known at which step Rab3-GEF and GAP are recruited in the SV recycling process. In addition, synapsins, identified as Rab3 effector proteins on vesicles, are considered key for SV clustering (Giovedì et al., 2004; Milovanovic and De Camilli, 2017; Shupliakov et al., 2011), and along with Rab3, they are employed as markers of mature, fusion competent SVs (Matteoli et al., 1991).

Rabconnectin-3a (Rbcn-3a), a large 340kDa (3,036 aa) protein encoded by $D x m / 2$, was originally identified as a Rab3-GEF and GAP interactor (Nagano et al., 2002). Rbcn-3a was found to be enriched in the brain and crude SV fraction, and localize at synapses (Nagano et al., 2002). Rbcn3a from rat brain synaptosomal preparation was found to interact with Rbcn-3b (encoded by the gene WDR7) (Kawabe et al., 2003) and several sub-units of the v-ATPase complex in a proteomics study (Li et al., 2012). To date, few studies addressed Rbcn-3a function using various model systems. (1) The yeast homolog of Rbcn-3a, Rav1, was found to regulate the assembly and activity of V-ATPase (Seol et al., 2001; Smardon and Kane, 2007; Smardon et al., 2002). Rav1 is part of the RAVE complex, and is involved, along with Rav2 and Skp1, in glucose-induced v-ATPase assembly (Seol et al., 2001), and has distinct interaction sites for Rav2p, Skp1p, $V_{1}$ E or $G$ and $V_{1} C$ subunit of V-ATPase complex (Smardon and Kane, 2007; Smardon et al., 2002). Loss of RAVE complex affected the transport between early endosome and pre-vacuolar compartment in yeast (Sipos et al., 2004). (2) The fly homolog of Rbcn-3a was reported to regulate Notch signalling and endocytic trafficking during development, primarily by acting through vATPase (Sethi et al., 2010; Yan et al., 2009). In the Rbcn-3a mutants, Notch receptor and other membrane proteins were trapped in the late endosome and the phenotype was similar to that of v-ATPase mutant VhaAC39. In a follow up study, Rbcn-3 was also found to regulate Notch signaling in mammalian cell lines (Sethi et al., 2010). Thus disrupting the v-ATPase or its regulator 
Rbcn-3 leads to an impaired Notch signaling in Drosophila. (3) In zebra fish, Rbcn-3a was found to stabilize the activity of v-ATPase on SVs at the ribbon synapse (Einhorn et al., 2012). SVs in the Rbcn-3a mutant hair cell failed to acidify fully and this was postulated to be due to fewer assembled v-ATPase. Further, Rbcn-3a was suggested to promote holoenzyme assembly as the distribution of $\mathrm{V}_{1} \mathrm{~A}$ subunit was altered in the mutant hair cells. The phenotype was again comparable to zebrafish mutants with defects in v-ATPase subunits (Nuckels et al., 2009). Rbcn$3 a$ was reported to regulate canonical Wnt signalling and endocytosis in the zebra fish neural crest by affecting endosomal fusion, but not acidification by v-ATPase in a more recent study in zebrafish (Tuttle et al., 2014). Endosomes in Rbcn-3a mutant or v-ATPase $V_{\text {oa1 }}$ subunit mutant acidified but remained immature. This suggested that Rbcn-3a may have a cell-type-specific function in the trafficking pathway (Tuttle et al., 2014).

In humans, alteration in $D M X L 2$ levels was found to cause a complex disorder named polyendocrine-polyneuropathy syndrome (PEPNS) with neurological and endocrine phenotype, defects in gonadotrophic axis, delayed onset of puberty, loss of gonadotropin-releasing hormone $(\mathrm{GnRH})$ neurons in hypothalamus and defects in $\mathrm{GnRH}$ network maturation (Tata et al., 2014, 2017). Mice deficient in DMXL2 (encoding for Rbcn-3a) were infertile due to the partial $\mathrm{GnRH}$ deficiency (Tata et al., 2014). Further, low Rbcn-3a expression in the brain resulted in a defective $\mathrm{GnRH}$ neuron maturation associated to a blunted response to kisspeptin, a hormone that controls $\mathrm{GnRH}$ secretion at puberty. The authors suggested that Rbcn-3a plays a key role in the postnatal maturation and the pubertal reactivation of the $\mathrm{GnRH}$ neuronal network through yet unknown mechanisms (Tata et al., 2017).

Based on the literature, we postulated that Rbcn-3a acts as a regulator of v-ATPase activity on SVs. Acidification process by v-ATPase is fundamental to trafficking processes at the synapse, including the SV recycling, thus understanding the function of Rbcn-3a may hold a key to addressing the question of how an endocytosed vesicle is converted into a mature fusion competent SV, ready for the next cycle of events. Also, it could explain the molecular mechanisms behind the phenotypes manifested in patients with altered $D M X L 2$ gene dosage. Apart from the proposed role in Rab3 activation and v-ATPase activity regulation, the specific function of Rbcn$3 a$ at the mammalian synapse remains largely uncharacterized. 


\section{RESULTS}

\section{SV-associated Rbcn-3a is found on clathrin coated pits and vesicles}

Rbcn-3a was originally identified as a peripheral SV protein that dissociated from SVs in the presence of high salt (Nagano et al., 2002). To study the function of Rbcn-3a, we cloned the human $D M X L 2$ gene into a mammalian expression vector that allows its co-expression with the enhanced green fluorescent protein (EGFP). The endogenous protein detected by anti-Rbcn-3a antibody co-localized well with SV peripheral protein Rab3 (Figure 1A), as described earlier (Nagano et al., 2002). Expression of EGFP-Rbcn-3a also gave a synaptic staining that overlapped with Rab3 at the synapses (Figure 1A). Interestingly, a punctate staining for Rbcn-3a was also observed in the neuronal soma (Figure 1B).

To confirm the localization of Rbcn-3a on the SVs, we isolated SVs from WT mouse brains and immunoblotted them for Rbcn-3a: we found that Rbcn3a is present at SVs like integral SV proteins synaptophysin and synaptobrevin-2/VAMP2 (Figure 1C). To find out at which stage of SV cycle Rbcn-3a gets recruited, we have isolated clathrin-coated vesicles (CCVs) from mouse brains, as recently reported (Farsi et al., 2018). Interestingly, Rbcn-3a was enriched in CCV samples as well (Figure 1C). We have shown that CCVs do not acidify despite they have a complete v-ATPase complex (Farsi et al., 2018), thus the role of Rbcn-3a may go beyond its putative role in v-ATPase regulation.

To examine if Rbcn-3a is already present at the clathrin-coated pits (CCPs), we colocalized Rbcn$3 a$ with clathrin light chain (C-LC) and adaptor protein 2 (AP2). Rbcn-3a localized to the few transient clathrin-positive puncta in wild-type fibroblasts (Figure 1D). To increase the chance to detect CCPs, we took advantage of a mouse fibroblast line that accumulates such structures, namely cells that lack all three dynamins (Ferguson et al., 2009; Park et al., 2013). Dynamin triple knock out (TKO) fibroblasts have arrested endocytic CCPs and are a useful model to study if a protein is recruited to the endocytic initiation site. Curiously, Rbcn-3a colocalized well with clathrin (Figure 1D) and $\sigma$ subunit of AP2 adaptor complex (Figure S1A) in dynamin TKO cells. Taken together, these findings suggest that Rbcn-3a, originally identified as a peripheral SV protein, localizes to the CCPs and is also present on CCVs. 


\section{Rbcn-3a colocalizes with v-ATPase subunits}

Rbcn-3a was reported to interact with several subunits of v-ATPase (Li et al., 2012). Rbcn-3a expressed together with a v-ATPase $V_{1} B$ subunit colocalized in the WT fibroblasts (Figure 1E). Interestingly, colocalization was more obvious in dynamin TKO fibroblast with accumulated CCP structures, implying that v-ATPase and Rbcn3a were present together at the forming endocytic pits (Figure 1E). To further verify the presence of v-ATPase complex subunits on CCPs, we expressed $V_{1} B$ and $V_{1} A$ together with endocytic marker proteins like $\sigma$ subunit of AP2 complex and C-LC respectively. The expressed v-ATPase subunits colocalized with endocytic markers and this was again evident in the dynamin TKO cells (Figure S1B-C). Given that $\mathrm{V}_{1}$ and $\mathrm{V}_{0}$ were shown to come together at the plasma membrane after fusion (Bodzęta et al., 2017), our data suggest that Rbcn-3a may have a role in stabilizing assembled v-ATPase during the clathrinmediated endocytic process. Further, the presence of v-ATPase subunits at the CCP complements our recent report that CCVs contain all the subunits of the v-ATPase complex, but do not acidify due to the blockage by the clathrin coat (Farsi et al., 2018). Although the clathrin coat alone could block CCV acidification, it is not yet known if other proteins are involved in this mechanism. Further, when and how the acidification of newly formed vesicle in the synapse is regulated is not known. To gain insight into the role of Rbcn-3a in SV recycling, we generated a mouse knock-out (KO) model system without Rbcn-3a in neurons.

\section{Mice without Rbcn-3a are lethal, yet neurons develop in culture and form synapses}

Rbcn-3a KO in mice leads to embryonic lethality (Tata et al., 2014). We thus used a conditional approach to obtain Rbcn-3a KO neurons in culture. Brains of newborn DMXL2 loxP/loxP mice were used to make primary cortical and hippocampal cultures and lentiviral mediated Crerecombination resulted in Rbcn-3a KO in culture (Figure S2). Loss of Rbcn-3a in this way did not affect overall development of neurons, as well as synapse formation. Immunofluorescence against a pre-synaptic active zone marker protein Bassoon and post-synaptic density marker Homer1 revealed no change in the number of synaptic appositions formed between WT and KOs (Figure 2A-B). We further checked if the overall number of synapses formed was affected in Rbcn-3a KO by measuring number of Bassoon-positive puncta in the volume of processes marked by MAP2 staining and this was also not changed in the Rbcn-3a KO neurons (Figure 2BD). 
Although Rbcn-3a neuronal cultures were morphologically comparable to controls, immunoblot analysis of neuronal lysates showed that in KOs, levels of several integral synaptic vesicle proteins (VAMP2, synaptophysin, $\mathrm{V}_{\text {o }}$ ) were significantly reduced when compared to controls (Figure 2E, quantification in Figure 2F). Rbcn-3b, the other identified subunit of Rbcn-3 complex however remained unchanged (Figure 2E, quantification in 2F). Taken together, we observe a reduction in the levels of integral SV proteins without changes in synapse formation and gross neuronal morphology in the absence of Rbcn-3a in neurons.

\section{Neurons lacking Rbcn-3a have altered synapse morphology and impaired synaptic transmission}

The overall reduction in vesicular marker proteins suggests a possible alteration in synapse morphology at the SV level. We therefore performed EM ultrastructure analysis of Rbcn-3a KO and WT neurons in culture (representative images are shown in Figure 3A-B). Rbcn-3a KO synapses revealed a characteristic phenotype with fewer SVs (Figure 3C) and an increase in endosome-like-structure (Figure 3D), whereas the coated structures per synapse (Figure 3E) remained unchanged. In addition, much sparser distribution of vesicles in the presynaptic terminal was noted in Rbcn-3a KO, whereas WTs had densely packed SVs in the synapses (Figure 3A-B). These data suggest that Rbcn-3a is likely required for proper presynaptic functioning and reduction in SV numbers due to the lack of this protein could impair SV dynamics at the synapse.

To address this, we first performed spontaneous fusion analysis from cultured hippocampal neurons to look at mEPSC (miniature excitatory postsynaptic current) by whole-cell patch clamp technique (Figure 3F). mEPSC recording measures the response elicited by a single vesicle release, wherein the frequency reflects vesicular release and amplitude depends on the activation of postsynaptic receptors. Lack of Rbcn-3a resulted in a reduction in spontaneous firing frequency (Figure $\mathbf{3 G}$ ) as well as a moderate reduction in the release amplitude (Figure $\mathbf{3 H}$ ). A change in amplitude reflects either a change in postsynaptic receptor activation/number, a change in vesicular size or fill state, or both. Since Rbcn-3a is known to interact with the proton pump on the vesicles that builds the electrochemical gradient for neurotransmitter refilling, it is plausible that the amplitude of release is altered because of changes in the vesicle NT content level. Taking 
the data together, Rbcn-3a deficiency results in a reduction in SV numbers and firing frequency of neurons, likely due to release of empty or partially filled vesicles.

\section{Synaptic vesicle recycling is affected with a delayed post stimulus recovery in Rben-3a KO neurons}

To assess the consequence of Rbcn-3a deficiency in SV recycling processes, we employed a pHluorin-based imaging strategy. vGLUT1-pHluorin, which has a pH sensitive pHluorin moiety tagged to vesicular glutamate transporter 1, was expressed in neurons and SV recycling was followed by live imaging (Figure 4A). Rbcn-3a KO neurons had a strong delay in post stimulus recovery after $10 \mathrm{~Hz}$ stimulation with $300 \mathrm{AP}$ (action potential) compared to the controls (Figure 4B). While the control neurons recovered to baseline values, the fluorescence decay in the KOs did not reach this point even after 4 minutes. The time constant of recovery after stimulation determined by an exponential fitting to the trace after $F_{\max }$ (during stimulation) was almost 4 fold

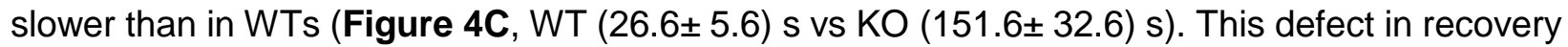
could be due to defective endocytosis of the probe after stimulation or due to a setback in the newly formed vesicle acidification. To check if it is an endocytosis problem, we used acid quenching strategy to measure the fraction of surface trapped probe. However, exposure to $\mathrm{pH}$ 5.5 buffer quenched similar proportion of pHluorin signal, suggesting that this is likely not due to defective endocytosis and surface trapping of the pHluorins (Figure 4D). The rate constant of exocytosis was determined by applying a $10 \mathrm{~Hz}$ stimulation with 300AP in the presence of $5 \mu \mathrm{M}$ bafilomycin $\mathrm{A} 1$ (blocks acidification and therefore recovery of vesicles completely) and was found to be increased in the KOs (Figure 4E-F). Interestingly, we observed an increased fluorescence of pHluorins in the baseline state even before stimulation in the Rbcn-3a KO (Figure 4G). Since the surface trapped fraction is not changed in the KOs, this suggests a possible scenario for alkaline trapping of pHluorins in recycling intermediates in the synapse of Rbcn-3a KO neurons.

\section{Lack of Rbcn-3a does not alter compensatory endocytosis in neurons}

To assess if the delayed recovery in Rbcn-3a KO neurons post stimulation is indeed due to a defect in endocytosis of membrane and proteins from the surface, we used an alternative approach to label vesicles with styryl dye FM1-43 (Ryan and Smith, 1995). FM dyes can enter and exit lipid bilayer without permeabilization and so have been used to follow the cycle of exo- 


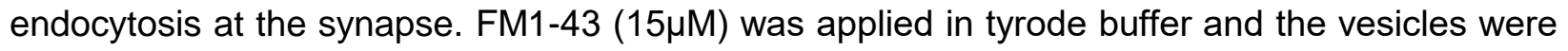
loaded during stimulation (600 AP) with the dye by endocytosis of dye-bound membrane (Figure 5A). After a 10 minute washout, the boutons were destained with a train of 1200 AP to release all the labeled vesicles and the fluorescence intensity in the boutons are plotted as a function of time (Figure 5B). The amount of dye internalized during activity was measured as the difference in fluorescence before and after unloading with the train of APs and this was not significantly different between the WT and KOs, suggesting that the membrane retrieval is not changed in the absence of Rbcn-3a (Figure 5C). The kinetics of de-staining was also comparable between the WTs and the KOs. Further, we checked several endocytic marker protein levels in Rbcn-3a KO and WT neurons and found no change in the total protein levels (Figure 5 D, quantification in E). In summary, no differences in the endocytic retrieval step and the protein machinery required for endocytosis, but a delay in pHluorin recovery kinetics point to a defect in re-acidification step after stimulation were observed. This is highly likely; given Rbcn-3a is a known interacting partner of the vesicular proton-pump (v-ATPase).

\section{Synaptic vesicle acidification is affected in Rbcn-3a KO neurons}

To determine whether Rbcn-3a regulates v-ATPase activity, we examined the acidification of SVs in culture and in vitro. Vesicle re-acidification kinetics can be estimated with pHluorin imaging in culture (Atluri and Ryan, 2006); however, this method reports bulk changes at the synaptic boutons and cannot resolve the changes happening at single vesicle level (Farsi et al., 2016). vATPase on the synaptic vesicles builds a concentration gradient $(\Delta \mathrm{pH})$ as well as an electrical gradient $(\Delta \Psi)$ across the vesicular membrane by pumping protons at the expense of ATP. These act as a driving force (combined electrochemical gradient $\Delta \mu_{\mathrm{H}_{+}}$) for the vesicular transporters to load NTs into the lumen of SVs (Farsi et al., 2017). Imaging based measurements of vesicular membrane potential and $\mathrm{pH}$ from isolated SVs and CCVs were used to dissect mechanism of vesicle acidification during the recycling process (Farsi et al., 2016; Farsi et al., 2018).

Since Rbcn-3a KO mice are embryonically lethal, we opted to use an immuno-isolation strategy to get functional SVs from cultured neurons and measure changes in $\mathrm{pH}$ and membrane potential at single vesicle level (Figure S3) and also measure directly if the lack of Rbcn-3a affects only vesicle acidification. We first verified by $E M$ if vesicles can be enriched using the immuno-isolation technique (Figure S3A-B). The vesicles bound to VAMP2 antibody on the beads were eluted 
efficiently using a control peptide (Figure S3C) and found to be structurally intact by EM, as well as retaining their functionality (Figure S3D-E). We labelled the isolated SV from WT and KO culture with the potentiometric dye VF2.1.Cl (Figure 6A), and measure their fluorescence change upon addition of ATP to determine their membrane potential (Farsi et al., 2016; Miller et al., 2012). Interestingly, change in fluorescence upon adding ATP was smaller in SVs from Rbcn-3a KO compared to WTs, thereby showing that the membrane potential is reduced in Rbcn-3a KO vesicles (Figure 6B-C). The distribution of membrane potential showed that the majority of Rbcn3a KO SVs did not acidify upon ATP addition (Figure 6D). Taken together, the data suggest that lack of Rbcn-3a affects only vesicle acidification during the recycling process, but does not alter endocytosis.

\section{Rbcn-3a promotes Rab3 stability and recruitment to SVs after recycling process}

We wanted to understand the consequence of lack of proper acidification in Rbcn-3a KO neurons. At the synapse, excessive membrane and proteins are internalized by endocytosis to form SVs locally, followed by vesicle acidification; NT refilling and then they are ready to participate in the next round of fusion events. Peripheral SV proteins like Rab3 and synapsin among others are recruited to newly formed vesicles after recycling, which modulate their function in the presynaptic mechanisms. Rbcn-3a was initially identified in a pull-down with Rab3-GEF, mediated by Rbcn3b from rat brain homogenates (Kawabe et al., 2003). Rab3-GEF helps the GDP-GTP exchange on Rab3, which can then be recruited on the newly (re-)formed vesicles. Since the fill state of a vesicle affects its participation in the fusion event, we wanted to check if recruitment of peripheral proteins (e.g. Rab3) can be a molecular correlate of this process. Hence we checked for the levels of Rab3 in Rbcn-3a KO neurons and their recruitment to SVs. Immunofluorescence analysis of total Rab3 intensity in neurons over a given volume of dendrite labeled with MAP2 showed a significant reduction in Rab3 signal in the KOs (Figure 7A, 7B). This suggests that Rbcn-3a is important for Rab3 stability as well as its recruitment to SVs during the recycling process at the synapse. This was further supported by a reduction in total protein level of peripheral proteins like Rab3 and synapsins in the KO neurons (Figure 7C, 7D). Collectively, these results show that impaired vesicle acidification due to the loss of Rbcn-3a leads to defective recruitment of peripheral SV marker protein Rab3, thereby affecting the recycling process at the synapse. 


\section{Rbcn-3a KO neurons accumulated lysosome-like structures}

The observed reduction in the SV number and levels of integral vesicular proteins in Rbcn-3a KO neurons prompted us to check if they are re-routed to the endo-lysosomal pathway instead of local recycling. Rbcn-3a was shown to affect the constitutive trafficking pathway in the cells, wherein the absence of Rbcn-3a caused the cargo or receptors to accumulate in several intermediate compartments along the pathway (Sethi et al., 2010; Tuttle et al., 2014; Yan et al., 2009). This suggested that Rbcn-3a could be involved additionally in the regulation of the constitutive trafficking route in the neurons. EM analysis of Rbcn-3a KO neurons showed an increase in endosome-like structures (Figure 3B, 3D). Interestingly, large dense lysosome-like structures were observed in the KO neurons (Figure 8A). Further, the level of lysosomal marker protein LAMP1 (lysosome associated marker protein 1) was increased in Rbcn-3a KO neurons, while early endosomal markers were not significantly altered (Figure 8B, quantification in C). Lysosomal accumulation was also detected by immunofluorescence analysis of LAMP1 in neuronal cell bodies (Figure 8D). This suggests that Rbcn-3a links several steps in the membrane trafficking pathway and regulate the recycling routes.

The results of this study lead us to postulate a model where Rbcn-3a acts as a v-ATPase quality controller that promotes stable v-ATPase assembly and activity, ensuring SVs acidify properly during SV recycling process, regulate Rab3 recruitment to SVs and links key steps in the trafficking pathway.

\section{DISCUSSION}

Acidification of SV lumen by v-ATPase is a necessary step in loading NTs into SVs (Edwards, 2007; Farsi et al., 2017; Goh et al., 2011; Saroussi and Nelson, 2009; Schenck et al., 2009). The regulatory mechanisms that ensure proper v-ATPase activity on SVs are unknown. We report here that Rbcn3a regulates v-ATPase activity and provide evidence for a role of Rbcn-3a in vesicle acidification and $S V$ recycling process at mammalian neuronal synapses. To understand its function as a v-ATPase regulator and its relevance for the SV recycling process, we characterized the function of Rbcn-3a at the neuronal synapse using a KO model system. We found Rbcn-3a not only on SVs, but also on CCVs and coated pits in mouse fibroblasts, occurring 
together with v-ATPase complex subunits. Vesicle acidification is impaired in KOs and the overall levels of v-ATPase subunits are reduced. Loss of Rbcn-3a in neurons reduces their spontaneous release frequency. Collectively, our data suggest that Rbcn-3a modulates neurotransmission in cultured neurons by promoting v-ATPase activity on SVs. The recovery after stimulation of pHluorin probe is delayed in Rbcn-3a KO neurons, but this is not due to a defect in the endocytic retrieval step. We observed that the consequence of the defective acidification is linked to the recruitment of peripheral proteins (e.g. Rab3) on SVs through Rbcn-3a in the neuronal synapse. Furthermore, Rbcn-3a loss affects the constitutive recycling route in neurons and results in accumulation of lysosome-like structures. Hence, we propose that Rbc3a modulates synaptic activity in neurons by stabilizing the v-ATPase assembly/activity on vesicles during the recycling process.

\section{Rbcn-3a is found on clathrin coated structures, together with the v-ATPase complex subunits}

In the recycling process, the stage at which a newly formed vesicle (by the retrieval process from PM or endocytic intermediate) undergo acidification is not yet known. Recently, a fully assembled v-ATPase with $V_{0}$ and $V_{1}$ subunits was detected in isolated CCVs (Farsi et al., 2018). The results of this work showed that the v-ATPase activity on the CCVs is blocked by the clathrin coat and suggested as a potential regulatory mechanism at the synapse. Rbcn-3a expression in neurons colocalized with SV marker proteins and this is in agreement with the pattern observed for Rbcn$3 a$ in the rat brain (Nagano et al., 2002). Interestingly, we also find Rbcn-3a on CCVs and endocytic coated pits, which localized with v-ATPase subunits. These results suggested that Rbcn-3a stabilizes v-ATPase assembly on forming endocytic vesicle and vesicle acidification is kept under check by the clathrin coat.

\section{Neurons develop and form synapses with altered morphology in the absence of Rbcn-3a}

Rbcn-3a KO in mice caused early embryonic lethality (Tata et al., 2014). Since v-ATPase activity is important for several developmental processesand as well, homologs of Rbcn-3 have been shown to regulate Notch and Wnt receptor signaling, we used a conditional approach in which Rbcn-3 was removed in cultured neurons (Yan et al., 2009, Tuttle et al., 2014). Synapse formation was not affected, yet the integral vesicle protein marker levels were reduced in Rbcn-3a KO neurons, suggesting that the presynaptic mechanisms are likely perturbed. v-ATPase levels were 
affected in Rbcn3a KO neurons, consistent with the finding that Rbc3a regulates $v$-ATPase activity via a mechanism involving stabilization of $V_{0}$ and $V_{1}$ subunits of the proton pump. $A$ reduction in vesicle protein level nicely correlated with the reduced number of SVs at the terminals of Rbcn-3a KO neurons in the EM analysis. This can be due to defective vesicle recycling locally, re-routing of vesicles along a different pathway in the cell, or both.

\section{Loss of Rbcn-3a results in defective SV recycling}

We used the pHluorin based strategy to follow presynaptic mechanisms in the Rbcn-3a neurons. Using pHluorin tagged to VGLUT1, we noted that the recovery after stimulation was delayed or slowed down in the absence of Rbcn-3a. The recovery is commonly defined as a two-step process (Sankaranarayanan and Ryan, 2000) and so the observed phenotype could be due to defective endocytosis or re-acidification process. It is curious to note that the observed recovery kinetics are comparable to that of wild-type hippocampal neurons with half maximal concentration of vATPase inhibitor bafilomycin A1 (Sankaranarayanan and Ryan, 2000). In that study, even a complete block of v-ATPase with $5 \mu \mathrm{M}$ bafilomycin A1 did not affect the endocytic retrieval of FM styryl dye in the neurons. Our findings that the rate as well as the amount of FM dye uptake is unaltered in Rbcn-3a KO neurons are well in line with the Sankaranarayanan and Ryan,2000. This was further supported by unchanged surface pool of vGLUT1-pHluorin and total protein levels of major endocytic factors in the KOs. Taken together, the data suggests that the observed difference in recovery is not due to a retrieval defect, but could be an acidification problem, where vesicles are trapped in an alkaline state.

\section{Rbcn-3a affects vesicle acidification and neurotransmission}

Re-acidification kinetics measured from imaging pHluorin expressing neurons seems to be much slower compared to the value reported by imaging at single vesicle resolution (Atluri and Ryan, 2006; Farsi et al., 2016). To by-pass the difficulty to separate endocytosis from vesicle acidification, we adapted single vesicle imaging to the Rbcn-3a KO neuronal system. V-ATPase is central to the electrochemical gradient that influences the NT filling in vesicles, and, includes the chemical $(\Delta \mathrm{pH})$ and the electrical gradient $(\Delta \psi)$ components. Assays to monitor both at the single vesicle level precisely have been developed (Farsi et al., 2016). Our findings showed that the membrane potential is reduced in the Rbcn-3a KO neurons, suggesting that the recovery phenotype is mostly due to defective vesicle acidification. Further, it would be important to 
measure the precise $\mathrm{pH}$ of vesicles in the $\mathrm{Rbcn}-3 \mathrm{a} \mathrm{KO}$ to determine the severity of the acidification problems.

The NT fill state is influenced by the electrochemical gradient and hence a problem with vesicle acidification can impact transmission process. Readouts of spontaneous vesicle fusion gives an idea about the vesicle NT fill state. The quantal release was reduced in VGLUT1-KO neurons (Wojcik et al., 2004), as well as neurons treated with v-ATPase blocker bafilomycin A1 (Zhou et al., 2000). Rbcn-3a KO neurons also showed a reduction in miniature excitatory postsynaptic current (mEPSC) frequency and a modest change in amplitude. A similar result was found in Rbcn-3a KO zebra fish hair cells, where the firing rate was reduced, but not to the extent imposed by bafilomycin A1 (Einhorn et al., 2012). Further evaluation of release properties will be essential to understand the role of Rbcn-3a in vesicle fusion process. Additionally, modification of available

probes to target the vesicular lumen and measure the vesicular NT content (e.g. glutamate sensor, (Marvin et al., 2013) will demonstrate whether the defective acidification and decreased activity are related to the NT filling step.

\section{Rbcn-3a function is essential for Rab3 recruitment to SVs and for trafficking pathways in neurons}

The consequence of defective acidification beyond the impact on NT fill state is not known. Besides, it is not yet clear how a newly (re-)formed vesicle by endocytic mechanisms undergoes transformation to be a fusion competent vesicle after the refilling step. To this end, we capitalized on the initial report that Rbcn-3a binds to Rab3 regulators, Rab3-GEF and Rab3-GAP (Kawabe et al., 2003; Nagano et al., 2002). Rab3 is important in membrane trafficking and regulates calcium triggered exocytosis (Pavlos and Jahn, 2011). In the absence of Rbcn-3a, recruitment of Rab3 to SVs is impaired and the overall protein level is also reduced. Thus, a putative v-ATPase regulator Rbcn-3a seems to be linking endocytosis, vesicle acidification and vesicle maturation to the formation of a fusion competent vesicle at the synapse.

EM analysis pointed to an accumulation of endosome-like and lysosome-like structures in the Rbcn-3a KO neurons. The homologs of Rbcn-3a have roles in endosomal maturation and receptor trafficking along endo-lysosomal pathways (Sethi et al., 2010; Tuttle et al., 2014; Yan et al., 2009). In addition, v-ATPase subunit levels were reduced in the KO neurons, consistent with suggested degradation of proton pump subunits due to trafficking defects. (Hurtado-Lorenzo et 
al., 2006; Oehlke et al., 2011). Further, the level of lysosomal protein marker, LAMP1, was increased in the KO neurons. Since v-ATPase has a housekeeping role in the constitutive trafficking pathway, the observed changes in the endo-lysosomal pathway could be due to a rerouting process in the absence of Rbcn-3a in neurons. Moreover, follow up studies will help to understand how the different recycling routes are linked through the action of Rbcn-3a and vATPase.

In summary, we report a novel role for Rbcn-3a in the regulation of SV acidification in mammalian neurons. Rbcn-3a seems to modulate the activity of the v-ATPase on vesicles, most likely through a mechanism that stabilizes v-ATPase assembly and activity. Rbcn-3a function seems to be linking the v-ATPase activity to trafficking events, thereby functioning as a molecular link between the different steps in the local recycling pathway and the constitutive trafficking pathway.

\section{ACKNOWLEDGEMENTS}

We thank the UMG animal facility, D. Schwitters and M. König for the excellent assistance. This work was supported by Schram-Stiftung T287/25457 and Deutsche Forschungsgemeinschaft (Emmy Noether Young Investigator Award MI-1702/1) to IM, SySy fellowship and GGNB bridging fund to SG. Conceptualization IM; Investigation and/or Analysis SG, AR, ZF, IM; Writing: SG and IM (all coauthors contributed to the final ms). Authors declare no competing financial interests.

\section{Figure Contribution:}

SG (Figure 1,2,3F-H,4-8, S1-2), AR (Figure 3A-B), ZF (Figure S3), IM (Figure 3C-E)

\section{REFERENCES}

Atluri, P.P., and Ryan, T.A. (2006). The Kinetics of Synaptic Vesicle Reacidification at Hippocampal Nerve Terminals. J. Neurosci. 26, 2313-2320.

Beyenbach, K.W., and Wieczorek, H. (2006). The V-type H+ ATPase: molecular structure and function, physiological roles and regulation. J. Exp. Biol. 209, 577-589. 
Bodzęta, A., Kahms, M., and Klingauf, J. (2017). The Presynaptic v-ATPase Reversibly Disassembles and Thereby Modulates Exocytosis but Is Not Part of the Fusion Machinery. Cell Rep. 20, 1348-1359.

Ceccarelli, B., Hurlbut, W.P., and Mauro, A. (1973). Turnover of Transmitter and Synaptic Vesicles at the Frog Neuromuscular Junction. J. Cell Biol. 57, 499-524.

Edwards, R.H. (2007). The neurotransmitter cycle and quantal size. Neuron 55, 835-858.

Einhorn, Z., Trapani, J.G., Liu, Q., and Nicolson, T. (2012). Rabconnectin3a promotes stable activity of the H+-pump on synaptic vesicles in hair cells. J. Neurosci. Off. J. Soc. Neurosci. 32, 11144-11156.

Farsi, Z., Preobraschenski, J., Bogaart, G. van den, Riedel, D., Jahn, R., and Woehler, A. (2016). Single-vesicle imaging reveals different transport mechanisms between glutamatergic and GABAergic vesicles. Science 351, 981-984.

Farsi, Z., Jahn, R., and Woehler, A. (2017). Proton electrochemical gradient: Driving and regulating neurotransmitter uptake. BioEssays 39, 1600240.

Farsi, Z., Gowrisankaran, S., Krunic, M., Rammner, B., Woehler, A., Lafer, E.M., Mim, C., Jahn, R., and Milosevic, I. (2018). Clathrin coat controls synaptic vesicle acidification by blocking vacuolar ATPase activity.

Ferguson, S., Raimondi, A., Paradise, S., Shen, H., Mesaki, K., Ferguson, A., Destaing, O., Ko, G., Takasaki, J., Cremona, O., et al. (2009). Coordinated Actions of Actin and BAR Proteins Upstream of Dynamin at Endocytic Clathrin-Coated Pits. Dev. Cell 17, 811-822.

Fischer von Mollard, G., Mignery, G.A., Baumert, M., Perin, M.S., Hanson, T.J., Burger, P.M., Jahn, R., and Sudhof, T.C. (1990). rab3 is a small GTP-binding protein exclusively localized to synaptic vesicles. Proc. Natl. Acad. Sci. 87, 1988-1992.

Giovedì, S., Darchen, F., Valtorta, F., Greengard, P., and Benfenati, F. (2004). Synapsin Is a Novel Rab3 Effector Protein on Small Synaptic Vesicles. J. Biol. Chem. 279, 43769-43779.

Goh, G.Y., Huang, H., Ullman, J., Borre, L., Hnasko, T.S., Trussell, L.O., and Edwards, R.H. (2011). Presynaptic regulation of quantal size: $\mathrm{K}^{+} / \mathrm{H}^{+}$exchange stimulates vesicular glutamate transport. Nat. Neurosci. 14, 1285-1292.

Heuser, J.E., and Reese, T.S. (1973). Evidence for recycling of synaptic vesicle membrane during transmitter release at the frog neuromuscular junction. J. Cell Biol. 57, 315-344.

Hnasko, T.S., and Edwards, R.H. (2012). Neurotransmitter Corelease: Mechanism and Physiological Role. Annu. Rev. Physiol. 74, 225-243.

Hurtado-Lorenzo, A., Skinner, M., Annan, J.E., Futai, M., Sun-Wada, G.-H., Bourgoin, S., Casanova, J., Wildeman, A., Bechoua, S., Ausiello, D.A., et al. (2006). V-ATPase interacts with 
ARNO and Arf6 in early endosomes and regulates the protein degradative pathway. Nat. Cell Biol. 8, 124-136.

Inoue, T., Wang, Y., Jefferies, K., Qi, J., Hinton, A., and Forgac, M. (2005). Structure and Regulation of the V-ATPases. J. Bioenerg. Biomembr. 37, 393-398.

Jefferies, K.C., Cipriano, D.J., and Forgac, M. (2008). Function, structure and regulation of the vacuolar $(\mathrm{H}+)$-ATPases. Arch. Biochem. Biophys. 476, 33-42.

Kawabe, H., Sakisaka, T., Yasumi, M., Shingai, T., Izumi, G., Nagano, F., Deguchi-Tawarada, M., Takeuchi, M., Nakanishi, H., and Takai, Y. (2003). A novel rabconnectin-3-binding protein that directly binds a GDP/GTP exchange protein for Rab3A small G protein implicated in $\mathrm{Ca2+-}$ dependent exocytosis of neurotransmitter. Genes Cells 8, 537-546.

Li, K.W., Chen, N., Klemmer, P., Koopmans, F., Karupothula, R., and Smit, A.B. (2012). Identifying true protein complex constituents in interaction proteomics: The example of the DMXL2 protein complex. PROTEOMICS 12, 2428-2432.

Mani, M., Lee, S.Y., Lucast, L., Cremona, O., Di Paolo, G., De Camilli, P., and Ryan, T.A. (2007). The dual phosphatase activity of synaptojanin1 is required for both efficient synaptic vesicle endocytosis and reavailability at nerve terminals. Neuron 56, 1004-1018.

Marvin, J.S., Borghuis, B.G., Tian, L., Cichon, J., Harnett, M.T., Akerboom, J., Gordus, A., Renninger, S.L., Chen, T.-W., Bargmann, C.I., et al. (2013). An optimized fluorescent probe for visualizing glutamate neurotransmission. Nat. Methods 10, 162-170.

Matteoli, M., Takei, K., Cameron, R., Hurlbut, P., Johnston, P.A., Südhof, T.C., Jahn, R., and Camilli, P.D. (1991). Association of Rab3A with synaptic vesicles at late stages of the secretory pathway. J. Cell Biol. 115, 625-633.

Miller, T.M., and Heuser, J.E. (1984). Endocytosis of synaptic vesicle membrane at the frog neuromuscular junction. J. Cell Biol. 98, 685-698.

Miller, E.W., Lin, J.Y., Frady, E.P., Steinbach, P.A., Kristan, W.B., and Tsien, R.Y. (2012). Optically monitoring voltage in neurons by photo-induced electron transfer through molecular wires. Proc. Natl. Acad. Sci. 109, 2114-2119.

Milovanovic, D., and De Camilli, P. (2017). Synaptic vesicle clusters at synapses: a distinct liquid phase? Neuron 93, 995-1002.

Mollard, G.F. von, Südhof, T.C., and Jahn, R. (1991). A small GTP-binding protein dissociates from synaptic vesicles during exocytosis. Nature 349, 79-81.

Murthy, V.N., and Stevens, C.F. (1998). Synaptic vesicles retain their identity through the endocytic cycle. Nature 392, 497-501.

Nagano, F., Kawabe, H., Nakanishi, H., Shinohara, M., Deguchi-Tawarada, M., Takeuchi, M., Sasaki, T., and Takai, Y. (2002). Rabconnectin-3, a Novel Protein That Binds Both GDP/GTP 
Exchange Protein and GTPase-activating Protein for Rab3 Small G Protein Family. J. Biol. Chem. 277, 9629-9632.

Nuckels, R.J., Ng, A., Darland, T., and Gross, J.M. (2009). The vacuolar-ATPase complex regulates retinoblast proliferation and survival, photoreceptor morphogenesis, and pigmentation in the zebrafish eye. Invest. Ophthalmol. Vis. Sci. 50, 893-905.

Oehlke, O., Martin, H.W., Osterberg, N., and Roussa, E. (2011). Rab11b and its effector Rip11 regulate the acidosis-induced traffic of V-ATPase in salivary ducts. J. Cell. Physiol. 226, 638-651.

Park, R.J., Shen, H., Liu, L., Liu, X., Ferguson, S.M., and Camilli, P.D. (2013). Dynamin triple knockout cells reveal off target effects of commonly used dynamin inhibitors. J Cell Sci 126, 53055312.

Pavlos, N.J., and Jahn, R. (2011). Distinct yet overlapping roles of Rab GTPases on synaptic vesicles. Small GTPases 2, 77-81.

Pfeffer, S.R., and Kelly, R.B. (1985). The subpopulation of brain coated vesicles that carries synaptic vesicle proteins contains two unique polypeptides. Cell 40, 949-957.

Raimondi, A., Ferguson, S.M., Lou, X., Armbruster, M., Paradise, S., Giovedi, S., Messa, M., Kono, N., Takasaki, J., Cappello, V., et al. (2011). Overlapping Role of Dynamin Isoforms in Synaptic Vesicle Endocytosis. Neuron 70, 1100-1114.

Rizzoli, S.O., and Betz, W.J. (2005). Synaptic vesicle pools. Nat. Rev. Neurosci. 6, 57-69.

Ryan, T.A., and Smith, S.J. (1995). Vesicle pool mobilization during action potential firing at hippocampal synapses. Neuron 14, 983-989.

Ryan, T.A., Reuter, H., and Smith, S.J. (1997). Optical detection of a quantal presynaptic membrane turnover. Nature 388, 478-482.

Sankaranarayanan, S., and Ryan, T.A. (2000). Real-time measurements of vesicle-SNARE recycling in synapses of the central nervous system. Nat. Cell Biol. 2, 197-204.

Saroussi, S., and Nelson, N. (2009). Vacuolar H(+)-ATPase-an enzyme for all seasons. Pflugers Arch. 457, 581-587.

Schenck, S., Wojcik, S.M., Brose, N., and Takamori, S. (2009). A chloride conductance in VGLUT1 underlies maximal glutamate loading into synaptic vesicles. Nat. Neurosci. 12, 156-162.

Seol, J.H., Shevchenko, A., Shevchenko, A., and Deshaies, R.J. (2001). Skp1 forms multiple protein complexes, including RAVE, a regulator of V-ATPase assembly. Nat. Cell Biol. 3, 384391.

Sethi, N., Yan, Y., Quek, D., Schupbach, T., and Kang, Y. (2010). Rabconnectin-3 Is a Functional Regulator of Mammalian Notch Signaling. J. Biol. Chem. 285, 34757-34764. 
Shupliakov, O., Haucke, V., and Pechstein, A. (2011). How synapsin I may cluster synaptic vesicles. Semin. Cell Dev. Biol. 22, 393-399.

Sipos, G., Brickner, J.H., Brace, E. j., Chen, L., Rambourg, A., Kepes, F., and Fuller, R.S. (2004). Soi3p/Rav1p Functions at the Early Endosome to Regulate Endocytic Trafficking to the Vacuole and Localization of Trans-Golgi Network Transmembrane Proteins. Mol. Biol. Cell 15, 3196-3209.

Smardon, A.M., and Kane, P.M. (2007). RAVE Is Essential for the Efficient Assembly of the C Subunit with the Vacuolar H+-ATPase. J. Biol. Chem. 282, 26185-26194.

Smardon, A.M., Tarsio, M., and Kane, P.M. (2002). The RAVE Complex Is Essential for Stable Assembly of the Yeast V-ATPase. J. Biol. Chem. 277, 13831-13839.

Takei, K. (1996). The synaptic vesicle cycle: a single vesicle budding step involving clathrin and dynamin. J. Cell Biol. 133, 1237-1250.

Tata, B., Huijbregts, L., Jacquier, S., Csaba, Z., Genin, E., Meyer, V., Leka, S., Dupont, J., Charles, P., Chevenne, D., et al. (2014). Haploinsufficiency of Dmxl2, Encoding a Synaptic Protein, Causes Infertility Associated with a Loss of GnRH Neurons in Mouse. PLoS Biol. 12.

Tata, B.K., Harbulot, C., Csaba, Z., Peineau, S., Jacquier, S., and Roux, N. de (2017). Rabconnectin-3 $\alpha$ is required for the morphological maturation of $\mathrm{GnRH}$ neurons and kisspeptin responsiveness. Sci. Rep. 7, 42463.

Tuttle, A.M., Hoffman, T.L., and Schilling, T.F. (2014). Rabconnectin-3a regulates vesicle endocytosis and canonical Wnt signaling in zebrafish neural crest migration. PLoS Biol. 12, e1001852.

Watanabe, S., Trimbuch, T., Camacho-Pérez, M., Rost, B.R., Brokowski, B., Söhl-Kielczynski, B., Felies, A., Davis, M.W., Rosenmund, C., and Jorgensen, E.M. (2014). Clathrin regenerates synaptic vesicles from endosomes. Nature 515, 228-233.

Wojcik, S.M., Rhee, J.S., Herzog, E., Sigler, A., Jahn, R., Takamori, S., Brose, N., and Rosenmund, C. (2004). An essential role for vesicular glutamate transporter 1 (VGLUT1) in postnatal development and control of quantal size. Proc. Natl. Acad. Sci. U. S. A. 101, 71587163.

Yan, Y., Denef, N., and Schüpbach, T. (2009). The vacuolar proton pump (V-ATPase) is required for Notch signaling and endosomal trafficking in Drosophila. Dev. Cell 17, 387-402.

Zerial, M., and McBride, H. (2001). Rab proteins as membrane organizers. Nat. Rev. Mol. Cell Biol. 2, 107-117.

Zhou, Q., Petersen, C.C., and Nicoll, R.A. (2000). Effects of reduced vesicular filling on synaptic transmission in rat hippocampal neurones. J. Physiol. 525 Pt 1, 195-206. 


\section{MAIN FIGURES}
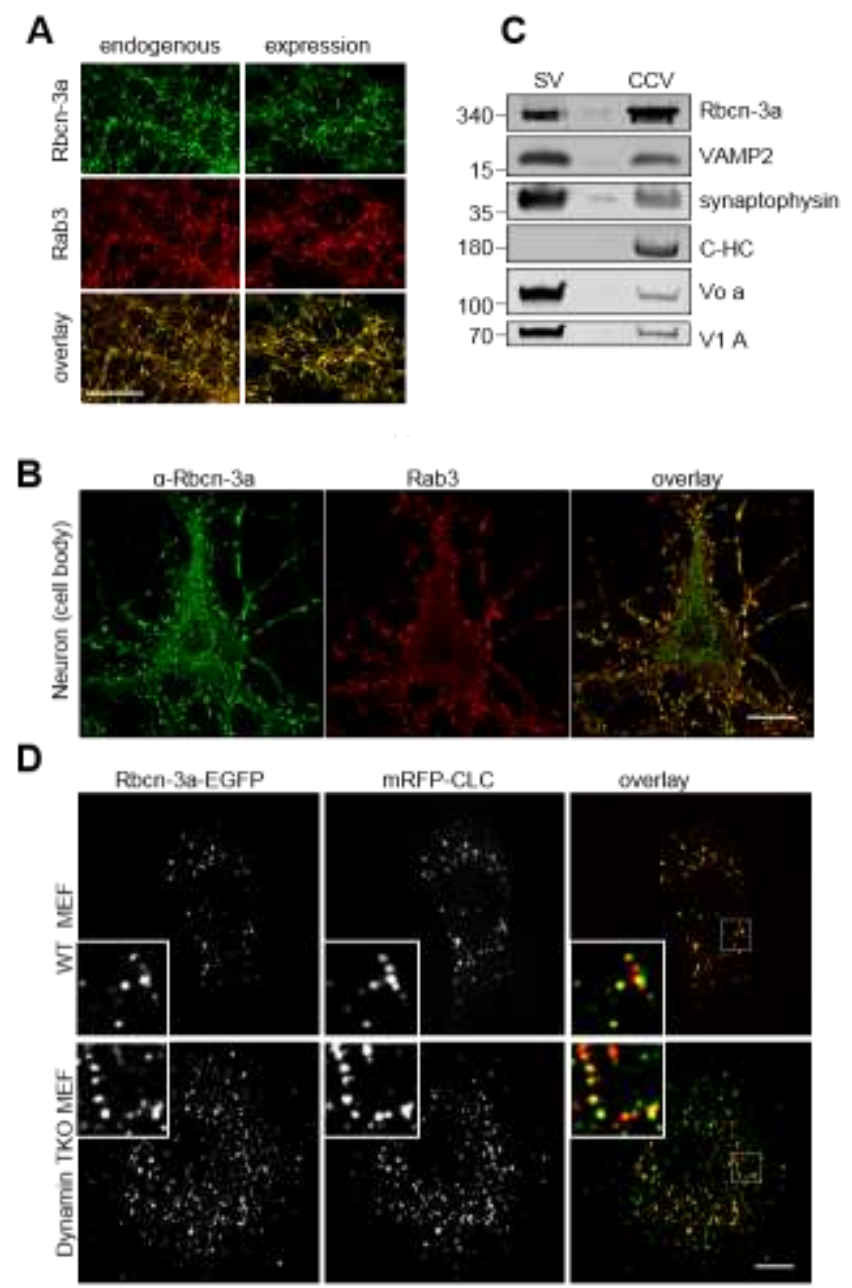

E

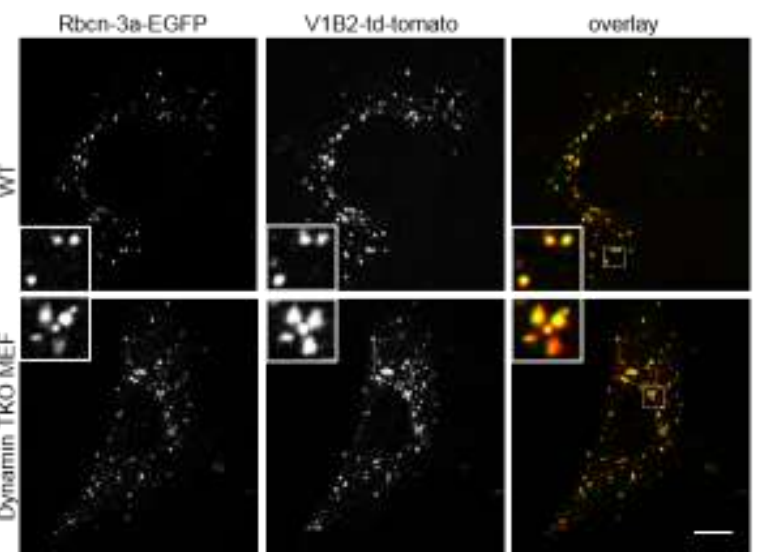

Figure 1. SV-associated protein Rbcn-3a is found on CCVs and localized at CCPs together with v-ATPase 
(A) Immunofluorescence against Rbcn-3a and peripheral SV protein Rab3 overlapped almost completely at the synapse. Expressed Rbcn-3a-EGFP was comparable to the endogenous protein detected by antibody and it overlapped with Rab3 signal at the synapse. Scale bar: $20 \mu \mathrm{m}$.

(B) Rbcn-3a had a punctate signal in the neuronal cell body apart from being enriched in the synapses. Scale bar: $10 \mu \mathrm{m}$.

(C) Immunoblots of isolated SVs and CCVs against Rbcn-3a, integral SV marker protein synaptophysin and synaptobrevin-2/VAMP2, clathrin-HC (C-HC) and v-ATPase subunits (same concentration of protein was loaded; 3 independent repetitions).

(D) Rbcn-3a co-localized with Clathrin-LC (C-LC) in control fibroblasts. In dynamin TKO MEFs with arrested CCPs, numerous puncta with Rbcn-3a signal overlapped with C-LC signal. Scale bar: $5 \mu \mathrm{m}$.

(E) Rbcn-3a co-localized with $v$-ATPase complex subunit $V_{1} B$ in control fibroblasts. Rbcn-3a localized together with $V_{1} B$ at the arrested CCPs in the dynamin TKO MEFs. Scale bar: $5 \mu \mathrm{m}$ ( 3 independent experiments, at least 30 cells per condition). 
A

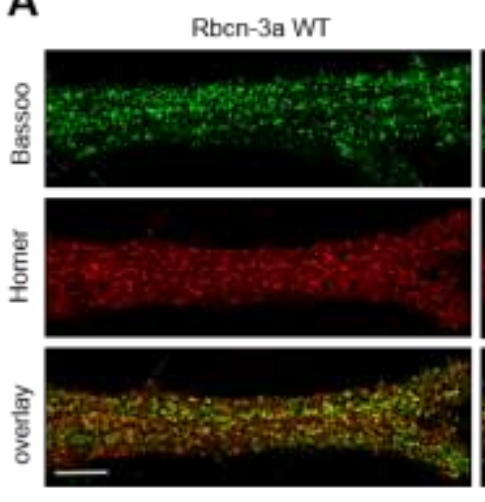

\section{C}
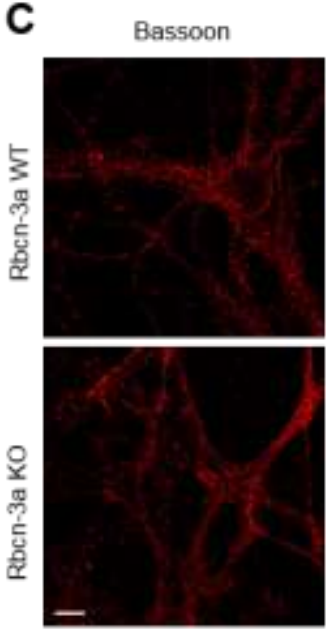

E

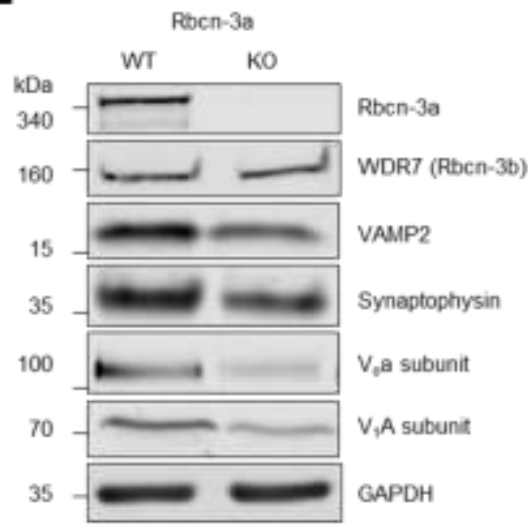

Rbcn-3a
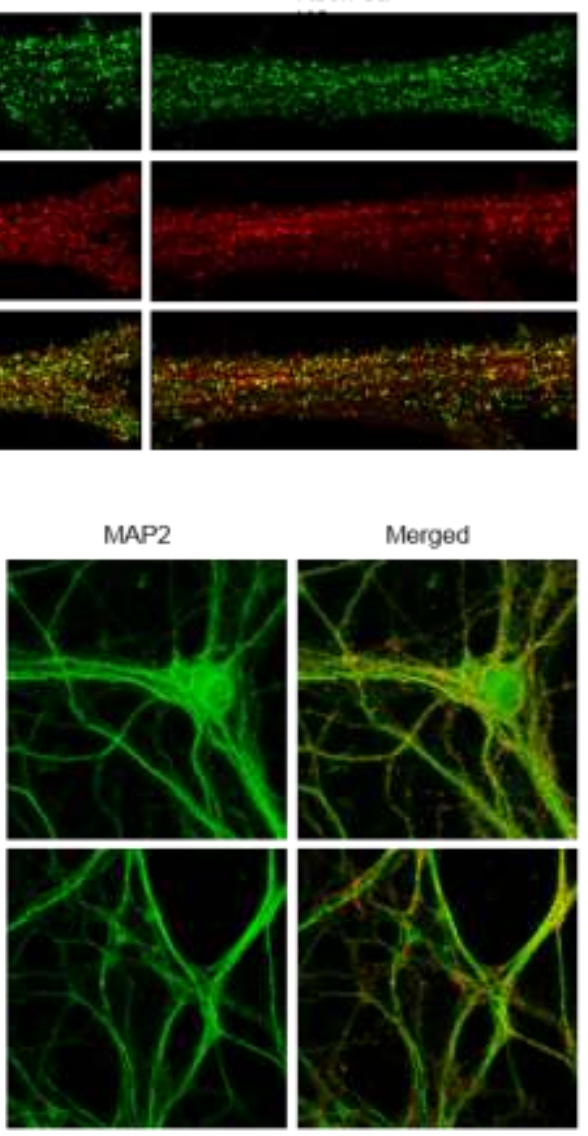

$\mathbf{F}$

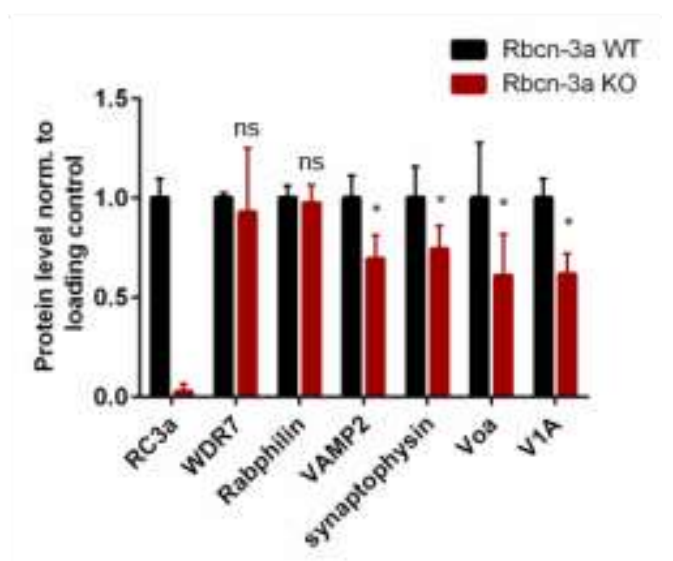

Figure 2. Rbcn-3a KO neurons in culture formed synapses, but the total levels of integral SV proteins were reduced 
(A) Immunofluorescence in Rbcn-3a WT and KO hippocampal neurons against Bassoon (pre-synaptic active zone protein) and Homer1 (postsynaptic density marker protein) showed a punctate signal that overlapped, indicating normal synapse formation. Scale bar: $10 \mu \mathrm{m}$.

(B) Ratio of Bassoon to Homer1 positive puncta was not significantly changed, suggesting that synapses were formed normally in the Rbcn-3a KO ( $n=3,30$ cells per condition).

(C) Rbcn-3a WT and KO neurons labeled with MAP2 to mark dendrites and stained with Bassoon to identify synapses. Scale bar: $10 \mu \mathrm{m}$.

(D) Number of synapses formed in Rbcn-3a KO neurons, measured by counting the Bassoon spots in the dendrite volume was not altered compared to the controls. ( $n=3,30$ cells per condition)

(E) Representative blots from analysis of neuronal lysates from Rbcn-3a WT and KOs used to check for the levels of various SV proteins.

(F) Levels of various integral SV proteins in neuronal lysates from Rbcn-3a WT and KOs. Levels of various SV proteins were reduced in the KOs. (4-10 repeats, Mann-Whitney test). 
A

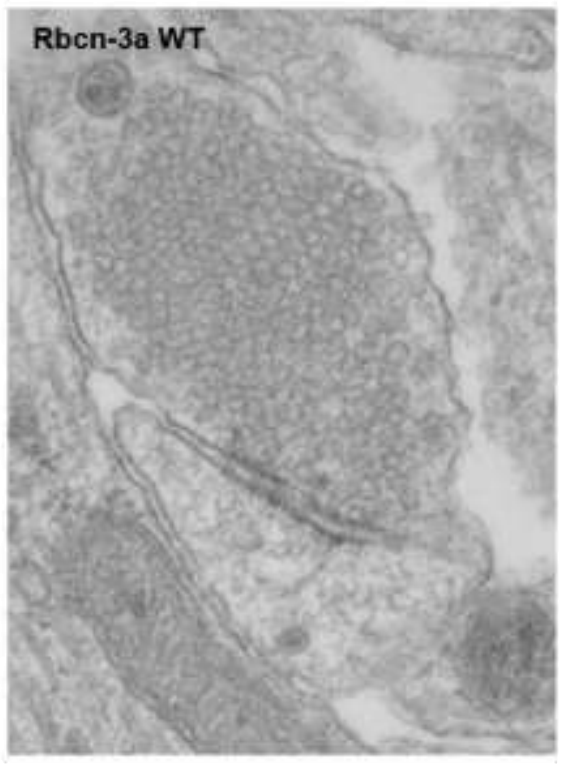

B

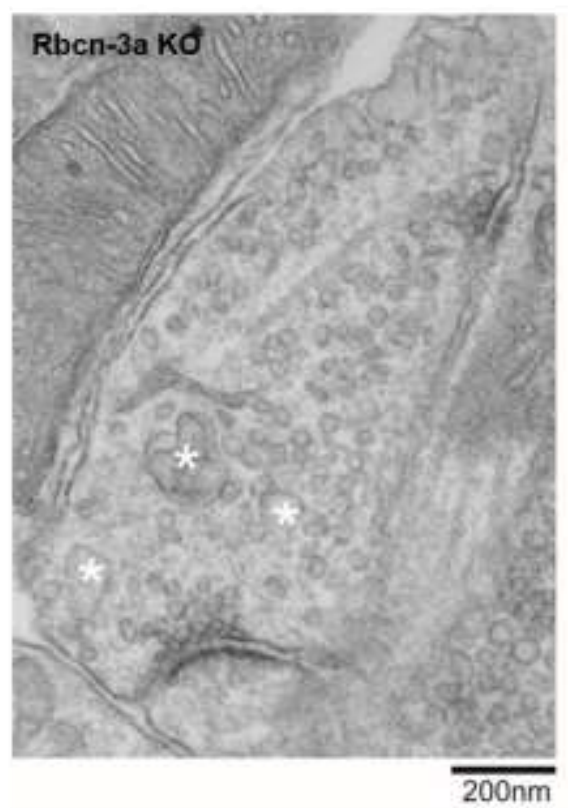

C

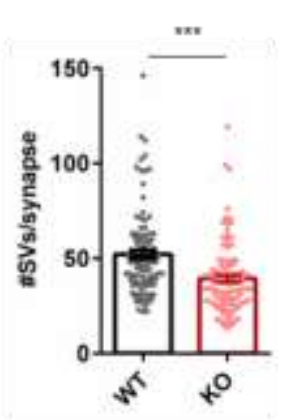

$\mathbf{F}$

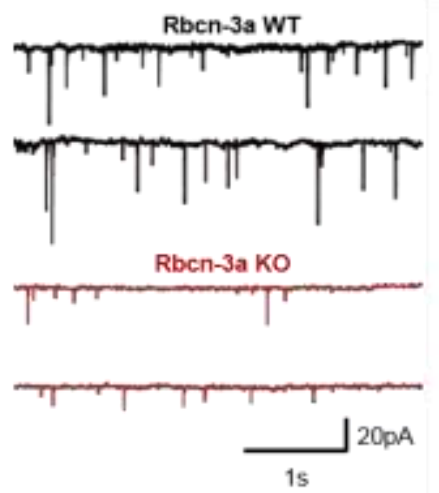

D

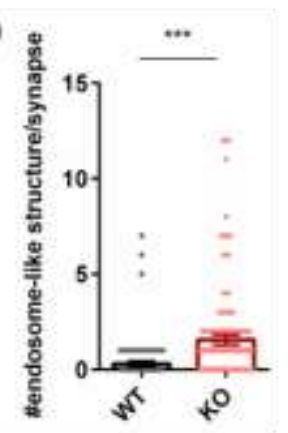

G

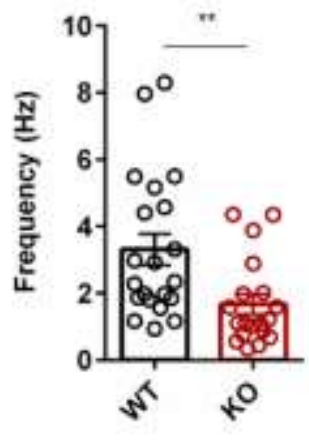

E

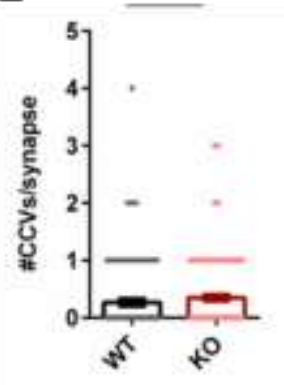

H

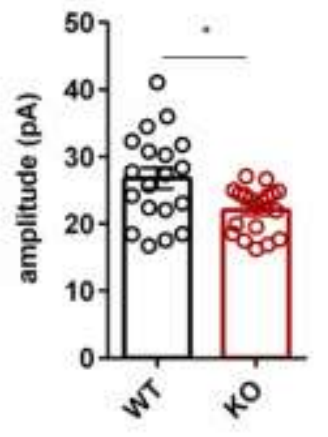

Figure 3. Neurons lacking Rbcn-3a had altered synapse morphology and impaired synaptic transmission 
(A and B) Electron micrographs showing ultrastructure of Rbcn-3a WT (A) and KO (B) synapse from hippocampal neuronal cultures (DIV15). The synaptic bouton is tightly packed with SVs in WT, whereas, in the KO, there are fewer SVs but more endosome-like-structures (denoted by *). Scale bar: $200 \mathrm{~nm}$.

(C-E) Morphometric analysis of SVs (C), endosome-like-structures (D) and CCV (E) in Rbcn-3a WT and KO synapses. Note a reduction in number of vesicles per synapse with concomitant increase in endosomelike structures in the KOs (marked by *). The number of CCV per synapse was unaltered ( 4 repetitions, 98 synapses)

(F) Representative traces showing spontaneous miniature EPSCs recorded from WT and Rbcn-3a KO hippocampal neurons (Scale bar: 1.2s and 20pA).

$(\mathrm{G}-\mathrm{H})$ Quantification of spontaneous quantal release showed that the mEPSC frequency (G) as well as the amplitude $(\mathrm{H})$ was reduced in Rbcn-3a KO neurons. ( $\mathrm{n}=3$ repetitions, 24 and 21 cells). 
A

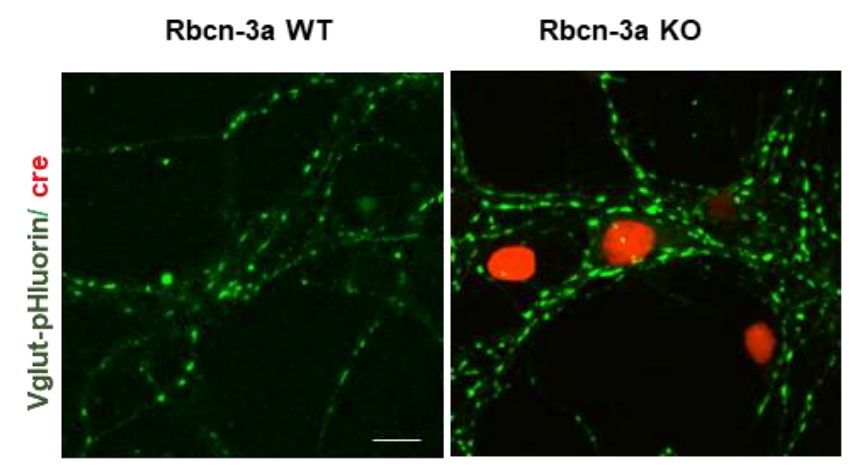

B

C

D
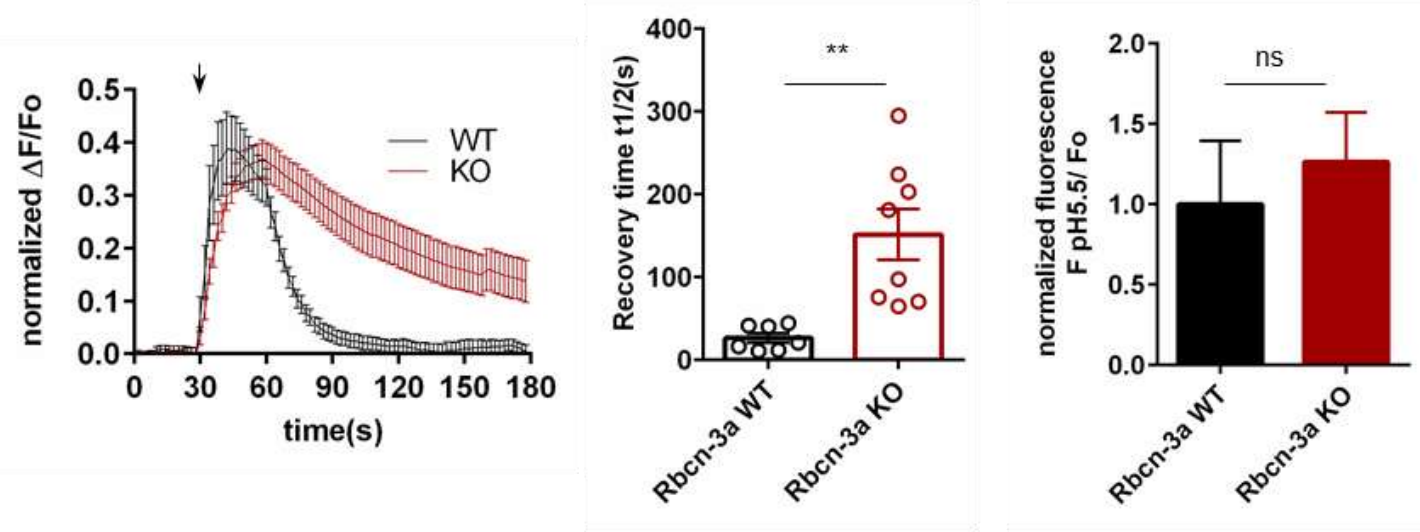

E

$\mathbf{F}$

G
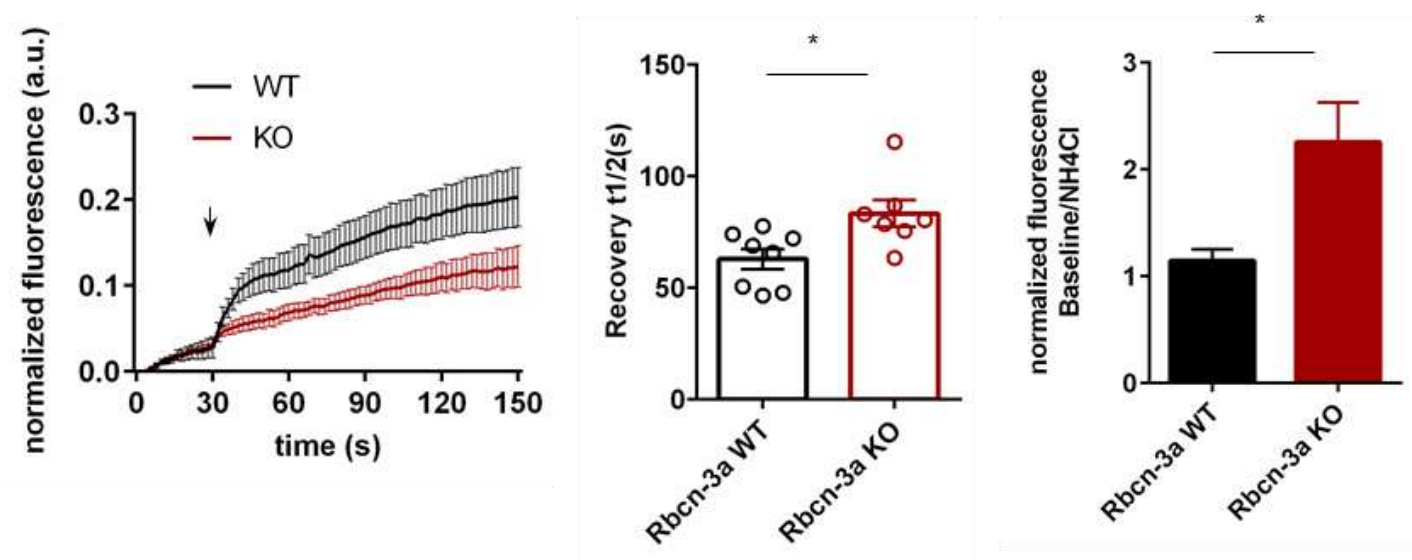

Figure 4. SV recycling was impaired due to a delayed post stimulus recovery in Rbcn-3a KOs 
(A) Illustration of the pHluorin-based imaging strategy to follow vesicle dynamics (left). Exemplary images of vGLUT1-pHluorin expressing WT and Rbcn-3a KO neurons. KO neurons had mRFP expressed in the nucleus (right). Scale bar: $10 \mu \mathrm{m}$

(B) Average vGLUT1-pHluorin fluorescence intensity changes with time during a $10 \mathrm{~Hz}$ stimulation in WT and $\mathrm{Rbcn}-3 \mathrm{a} \mathrm{KOs}$ (onset of stimulus is indicated by an arrow). The post stimulus recovery was delayed in the KOs. Values shown are mean \pm SEM. (3 repetitions, 30-40 boutons from 10-12 coverslips were measured).

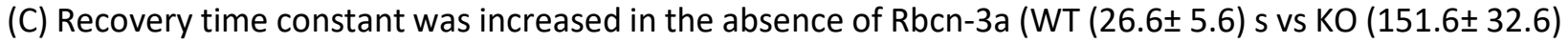

s). Time constants were obtained by fitting the traces to an exponential function.

(D) Fraction of pHluorin signal quenched by $\mathrm{pH} 5.5$ wash as a ratio of total fluorescence $\left(\mathrm{NH}_{4} \mathrm{Cl}\right.$ treatment) was similar between the conditions.

(E) Average traces of normalized response to $10 \mathrm{~Hz}$ stimulus in the presence of $5 \mu \mathrm{M}$ bafilomycin $\mathrm{A} 1$ to block recovery completely. (8 WT and $7 \mathrm{KOs,} \mathrm{arrow} \mathrm{indicates} \mathrm{the} \mathrm{onset} \mathrm{of} \mathrm{stimulus).}$

(F) The rate constant of exocytosis, determined by fitting the response in the presence of bafilomycin A1 to a first order exponential function was increased in the Rbcn-3a KO neurons.

(G) Ratio of pHluorin fluorescence during baseline condition compared to the total fluorescence revealed by $\mathrm{NH}_{4} \mathrm{Cl}$ treatment, signifying alkaline trapping of pHluorin moiety in Rbcn-3a KOs. 
A

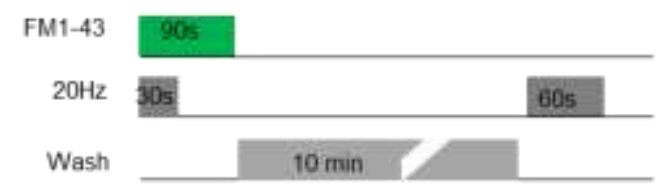

B

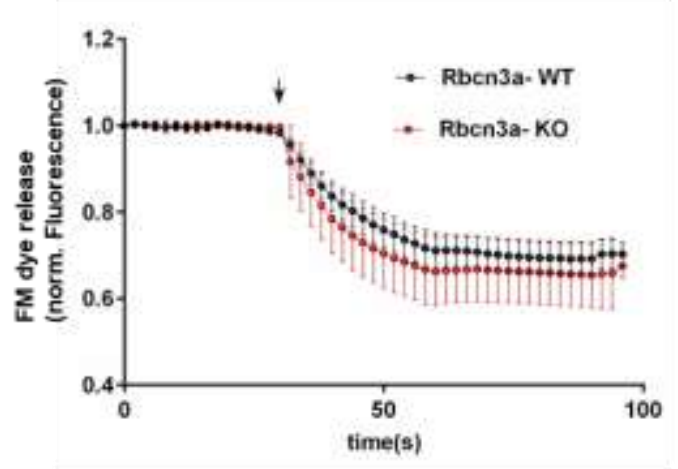

C

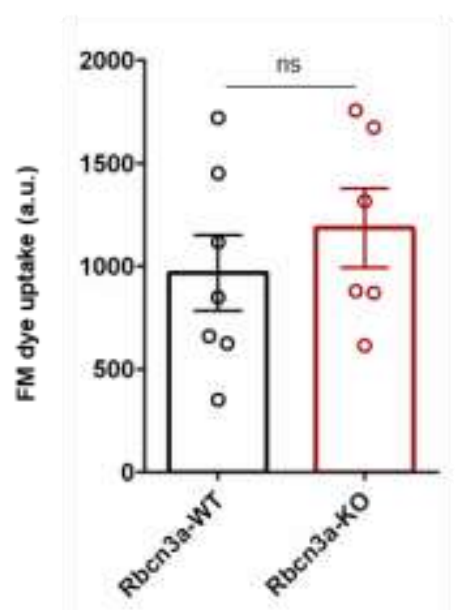

D

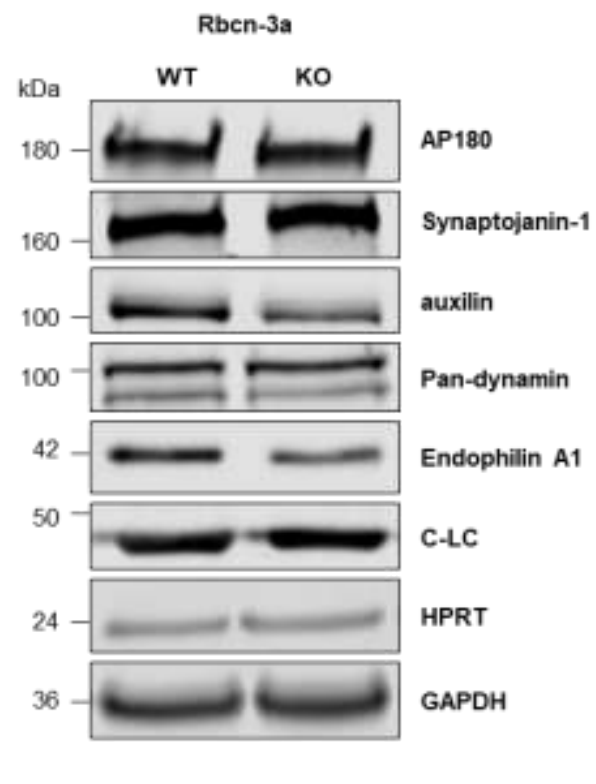

$\mathbf{E}$

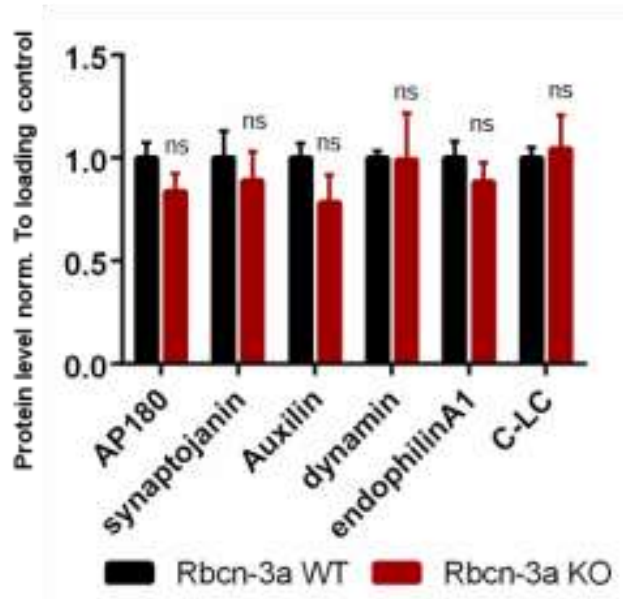

Figure 5. Compensatory endocytosis was not changed in Rbcn-3a KO neurons

(A) Schematic of a protocol used for FM 1-43 labeling of vesicle to measure endocytosis during activity. Neurons were stimulated with 600AP to label the entire recycling pool of vesicles using a $20 \mathrm{~Hz}$ stimulus. The excess dye was washed off immediately after loading and followed by another 10 minutes wash with Tyrode buffer. Neurons were stimulated again with 1200 AP to release all the loaded dye.

(B) Fluorescence decay of FM dye unloading from averaged WT and KO neurons as a function of time during the 1200 APs.

(C) FM dye uptake was not changed in Rbcn-3a KO neurons compared to the controls, indicating no change in compensatory endocytosis. 
(D) Representative immunoblots for proteins involved in endocytosis process tested in lysates from Rbcn3a KO and WT neurons.

(E) Levels of endocytic proteins were not significantly changed due to the lack of Rbcn-3a in neurons (410 repeats, Mann-Whitney test).

A

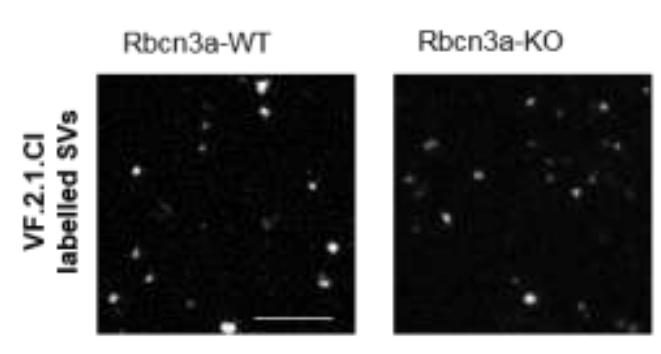

C

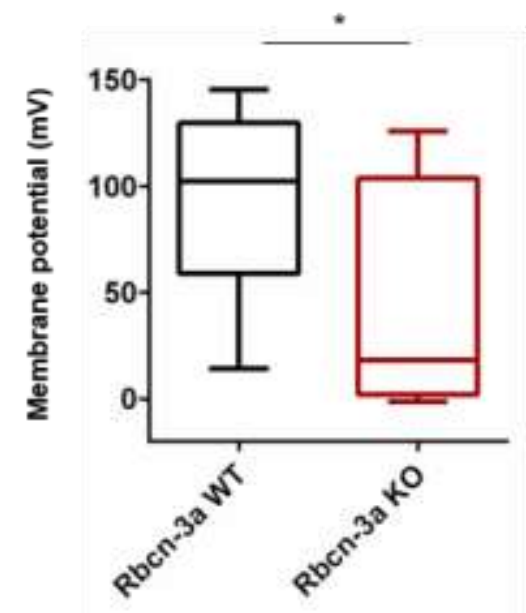

B

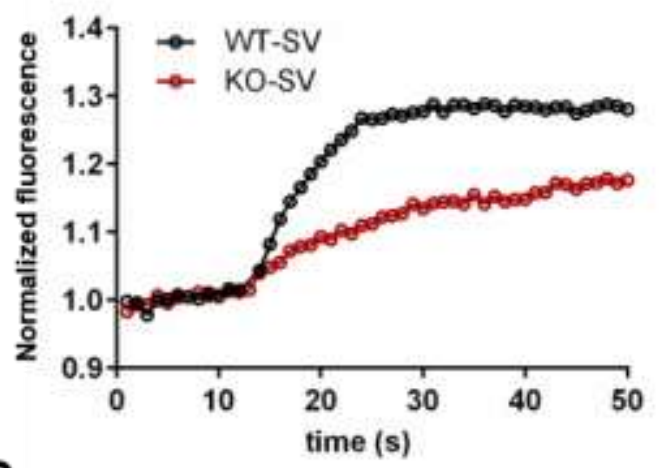

D

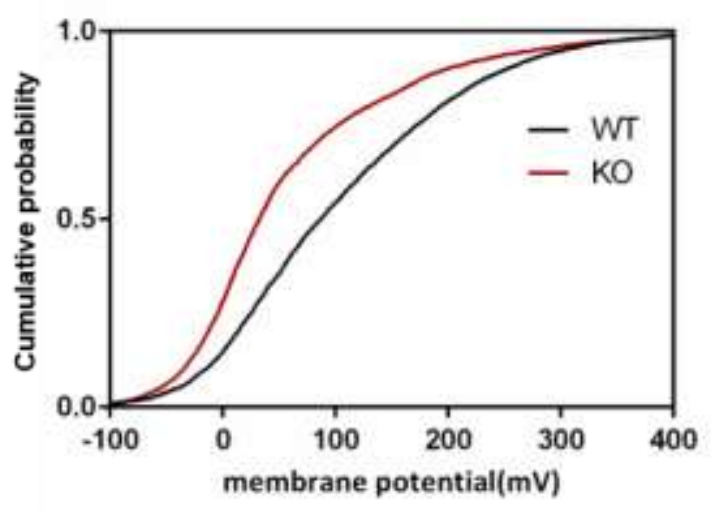

Figure 6. SV membrane potential was reduced in the absence of Rbcn-3a

(A) Representative images of VF2.1.Cl labeled SVs isolated from Rbcn-3a KO and WT neurons using TIRF microscopy. Scale bar: $4 \mu \mathrm{m}$.

(B) Averaged fluorescence response of VF2.1.Cl labeled SVs over time. Upon addition of ATP, the increase in fluorescence of the potentiometric dye observed in KO was smaller compared to the SVs from control neurons. (10 WT and 7 KO experimental replicates).

(C) Membrane potential of single SVs from Rbcn-3a KO was smaller than WT. Average value calculated from SVs of each replicate is presented by a box plot.

(D) Cumulative probability distribution of membrane potential of WT and KO SVs computed from fluorescence changes using VF2.1.Cl sensor show that majority of SVs in the KOs did not acidify upon ATP addition. 
A
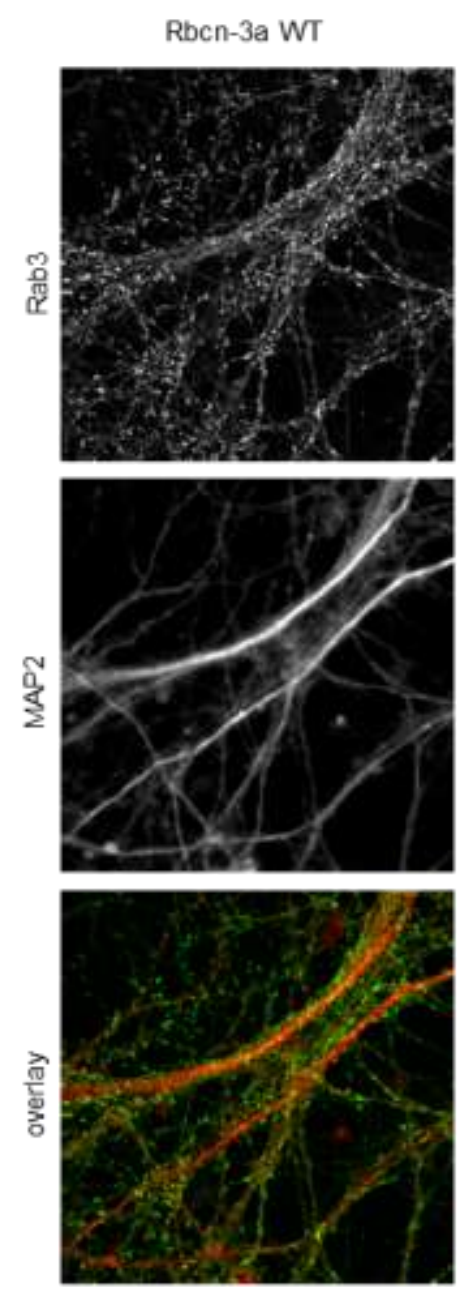

Rbcn-3a KO
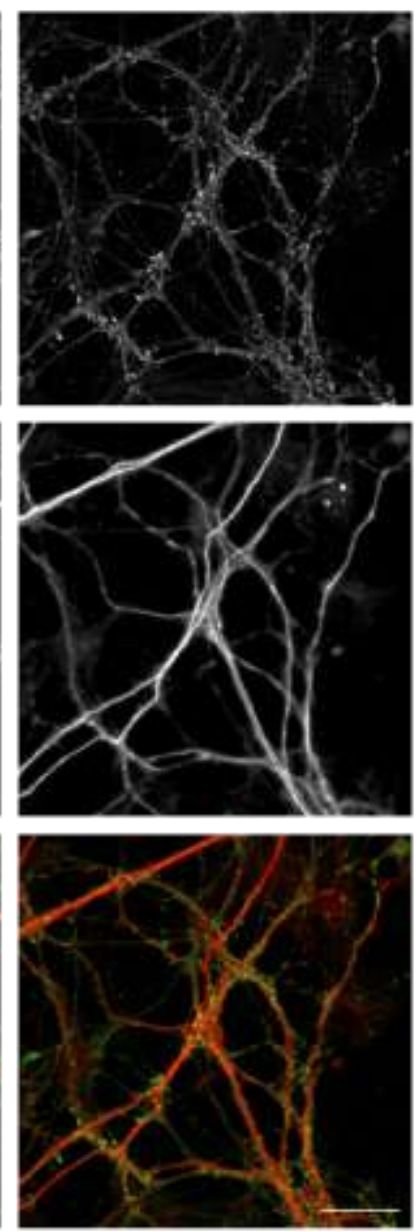

B

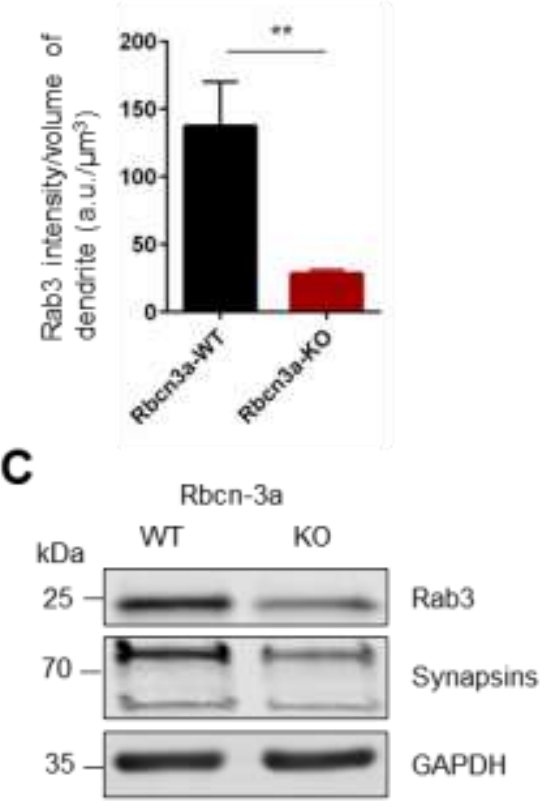

D

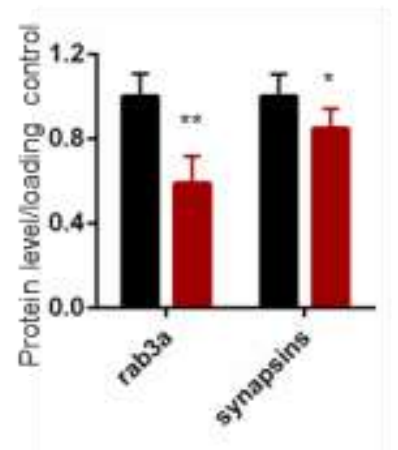

Figure 7. Loss of Rbcn-3a reduced the Rab3 stability and recruitment on SVs at the synapses

(A) Representative images of immunofluorescence in Rbcn-3a WT and KO neurons against Rab3, colabeled with MAP2. Scale bar: $10 \mu \mathrm{m}$.

(B) Quantification of Rab3a fluorescence signal in a given volume of the dendrite marked by MAP2 showed a reduction in Rbcn-3a KO neurons compared to WTs. ( $n=40,4$ independent repeats)

(C) Representative immunoblots against Rab3, synapsin and loading control (GAPDH) on WT and KO neuronal lysates.

(D) The levels of peripheral SV marker proteins were reduced in neurons lacking Rbcn-3a. (10 repeats). 
A

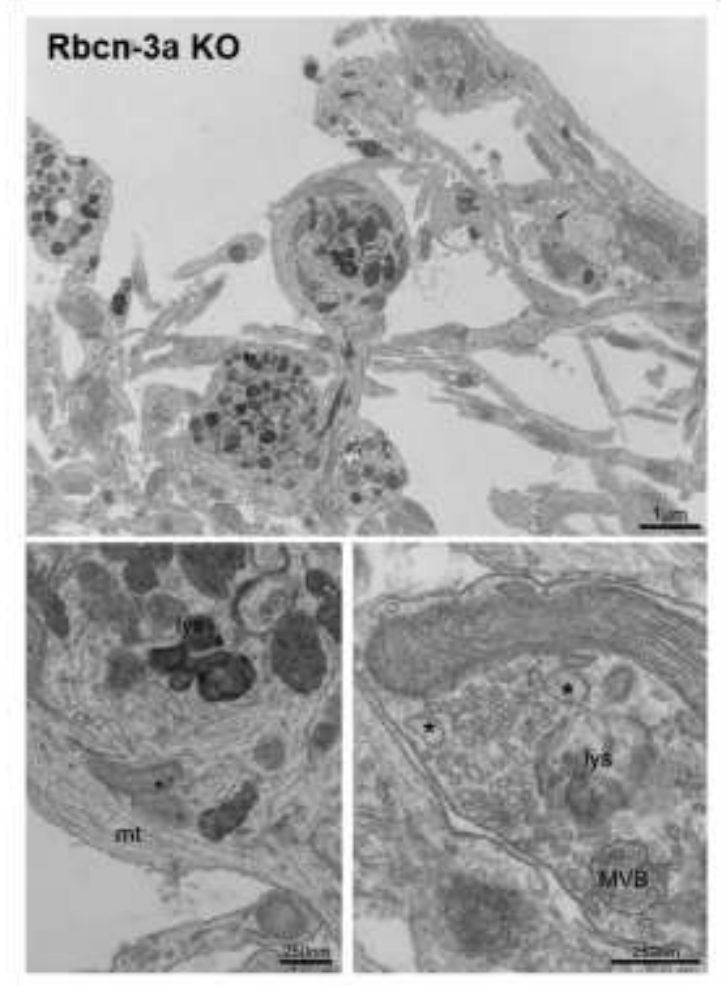

B

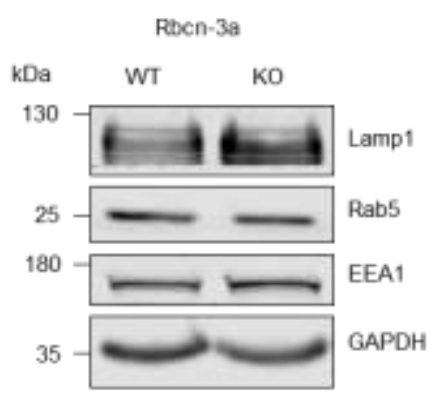

C

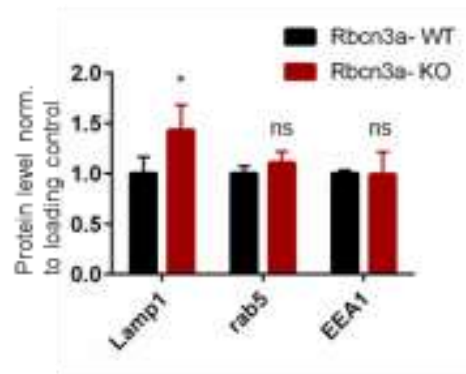

D
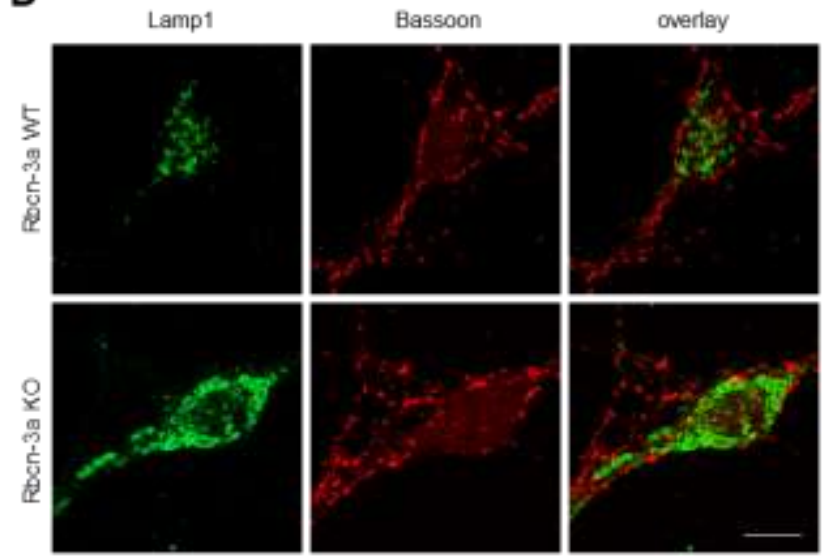

Figure 8. Rbcn-3a KO neurons accumulated lysosome-like structures

(A) Low magnification EM image showed increased accumulation of lysosome-like structures in Rbcn-3a KO neurons (scale bar: $1 \mu \mathrm{m}$ ). Higher magnification images below shows an axonal swelling, with selective accumulation of lysosome-like structures (scale bar: $250 \mathrm{~nm}$; endosome-like structures are indicated by *, MVB: multi-vesicular bodies, lys: lysosome-like structures). 
(B) Representative immunoblots against endo-lysosomal marker proteins Lysosomal-associated membrane protein 1 (Lamp1), rab5 and early endosomal marker 1 (EEA1). GAPDH was used as a loading control.

(C) The levels of Lamp1 were increased in Rbcn-3a KO neurons, while the early endosomal markers were not significantly changed. (4 independent repeats).

(D) Representative images of immunofluorescence in Rbcn-3a WT and KO neurons against Lamp1, colabeled with Bassoon and MAP2. Lamp1 positive structures are accumulated in the neuronal cell bodies in the KOs. Scale bar: $10 \mu \mathrm{m}$.

\section{METHODS}

\section{Transgenic mice and primary neuronal cultures}

All animal experiments were performed according to the European Guidelines for animal welfare (2010/63/EU). DMXL2 $2^{\text {loxploxp }}$ line (described in (Tata et al., 2014) was established and bred following the guidelines of ZTE animal facility. Animals were housed in breeding rooms with ad libitum food and water. P0/P1 DMXL2 ${ }^{\text {loxplloxp }}$ pups were used to prepare primary neuronal culture. $\mathrm{KO}$ was obtained by adding Cre recombinase lentivirus to neuronal cultures between days-invitro (DIV) 2-4. Primary neurons were transfected by electroporation (Amaxa TM 4D-Nucleofector X Unit, Lonza, Switzerland) or using Lipofectamine 2000 (Invitrogen) to express different proteins and detailed in the supplementary data.

\section{Cell Culture}

Cells (Mouse embryonic fibroblasts (MEFs) and HEK) were cultured in Dulbecco Modified Eagle's Medium (DMEM) medium containing $10 \%$ fetal bovine serum (FBS) and $1 \%$ penicillin/streptomycin. The cells were passaged at $70-80 \%$ confluence and used for the various experiments. Dynamin TKO was obtained by tamoxifen induced KO in cells in culture as described in Ferguson et al., 2009; Park et al., 2013. MEFs were transfected by electroporation (Amaxa TM 4D-Nucleofector X Unit, Lonza, Switzerland) for live imaging experiments, as detailed in the supplementary data.

\section{Biochemical methods}

Neuronal lysates were prepared by scraping the cells in $200 \mu \mathrm{L}$ lysis buffer (1\%SDS, $25 \mathrm{mM}$ Tris $\mathrm{pH} 7.4,150 \mathrm{mM} \mathrm{NaCl}, 1 \mathrm{mM}$ EDTA, supplemented with 1X protease and phosphatase inhibitor). The sample was quickly frozen using liquid nitrogen until further use. The neurons were lysed by 
passing the sample through 24 gauge syringe 15-20 times before proceeding with Western blotting. Synaptic vesicles were isolated from wild type mice according to the protocol described in Farsi et al., 2016 and SVs were stored in sucrose buffer by snap freezing. Clathrin-coated vesicle isolation was done according to the protocol mentioned in Farsi et al., 2018. The frozen vesicles were defrosted on ice and equal protein concentration of SV and CCV were used for the immunoblotting experiments.

\section{Immunocytochemistry}

Immunofluorescence staining was performed and sample was imaged on Zeiss LSM 800 confocal microscope. Cells were washed in pre-warmed PBS and fixed in $4 \%$ paraformaldehyde (PFA) containing $1 \%$ sucrose in PBS at $4^{\circ} \mathrm{C}$ overnight or RT for 1 hour. The free aldehyde was quenched with $50 \mathrm{mM} \mathrm{NH}_{4} \mathrm{Cl}$ for 20 minutes at RT. Further, the cells were permeabilized $(0.1 \%$ triton $x-100)$ and blocked for 1 hour in blocking solution (3\%BSA, 0.1\% cold fish gelatin, $1 \%$ goat serum in PBS) to prevent unspecific binding. The neurons were labeled with primary antibody in blocking buffer at $4^{\circ} \mathrm{C}$ overnight. After washing $3 \times 10$ minutes in PBS, the coverslips were treated with corresponding species secondary antibodies for 1 hour at room temperature in dark conditions. The samples were washed 3 X 10 minutes in PBS to remove non-bound signal and embedded on glass slides using Mowiol ${ }^{\circledR} 4-88$ mounting medium.

\section{Electron microscopy}

Electron microscopy experiments and analysis was done as previously described in Raimondi et al., 2011. Hippocampal neurons were fixed in $1.3 \%$ glutaraldehyde prepared in $66 \mathrm{mM}$ sodium cacodylate buffer and the samples were processed for TEM imaging. Data from 4 individual experiments were quantified using imageJ.

\section{Electrophysiology}

Spontaneous activity was measured from dissociated hippocampal neurons plated at a density of 75000-90000 cells /coverslip as described in Milosevic et al., 2011. Whole-cell patch clamp recordings from the neurons were acquired using EPC10 amplifier (HEKA Electronik, Germany) with a holding potential of $-70 \mathrm{mV}$. All the recordings were done at room temperature and patch master software was used for data acquisition. The extracellular medium used contained the following: $140 \mathrm{mM} \mathrm{NaCl}, 2.4 \mathrm{mM} \mathrm{KCl}, 2 \mathrm{mM} \mathrm{MgCl}$, 2 mM CaCl $2,14 \mathrm{mM}$ glucose, $10 \mathrm{mM} \mathrm{HEPES}$, 
pH 7.4 (300-310 mOsm) and additionally $20 \mu \mathrm{M}$ bicuculline, $2 \mu \mathrm{M}$ strychnine, $1 \mu \mathrm{M}$ TTX and 50 $\mu \mathrm{M}$ d-AP5 were added to isolate mini events (lack of evoked potential was verified after each recording by eliciting an EPSC with a brief $2 \mathrm{~ms}$ depolarization to $0 \mathrm{mV}$ ). Patch pipette resistance was between 2.5-5M $\Omega$ and the resistance was compensated up to $60 \%$. The intrapipette solution contained the following ( $136 \mathrm{mM} \mathrm{KCl}, 18 \mathrm{mM}$ HEPES, $15 \mathrm{mM}$ creatine phosphate, $4 \mathrm{mM} \mathrm{Na}$-ATP, 4.6 $\mathrm{mM} \mathrm{MgCl}, 1 \mathrm{mM} \mathrm{EGTA}$, and $50 \mathrm{U} / \mathrm{mL}$ phospocreatine kinase (300 mOsm), pH 7.3). Spontaneous events were identified and analyzed with Mini Analysis program (Synaptosoft).

\section{SV recycling by FM styryl dyes}

Styryl dye FM 1-43 was used to measure vesicle endocytosis; following the protocol described in (Mani et al., 2007). Briefly, synaptic boutons were labeled with $15 \mu \mathrm{M}$ FM1-43 in Tyrode buffer by $600 \mathrm{AP}$ to label the releasable vesicle pool. After a 10 minute washout, the neurons were stimulated again with 1200AP to measure the total amount of dye internalized. Images were analyzed using ImageJ, where 40-50 ROIs were selected per repetition and the intensity changes were noted over time. The intensity at any time point was normalized to $F_{0}$ and the average intensity was calculated from the first 10 frames. The rate of decay of fluorescence was fitted to a single exponential function to measure the endocytosis rate.

\section{SV recycling with vGLUT1-pHluorin}

Optical imaging of vesicle dynamics was performed using PerkinElmer UltraVIEW VoX system (Perkin Elmer, Waltham, Massachusetts, USA). SV dynamics were measured using a $\mathrm{pH}$ sensitive pHluorin probe, tagged with a luminal domain of an integral SV protein (e.g. vGLUT1) as described in Milosevic et al., 2011. vGLUT1-pHluorin under chicken beta actin promoter was expressed in neurons and imaged between DIV 14-18. Coverslips were mounted on imaging chamber, maintained in a temperature controlled chamber at $34-37^{\circ} \mathrm{C}$ and perfused with Tyrode buffer (119 mM NaCl, $5 \mathrm{mM} \mathrm{KCl}, 25 \mathrm{mM}$ HEPES buffer, $2 \mathrm{mM} \mathrm{CaCl}_{2}, 2 \mathrm{mM} \mathrm{MgCl}_{2}, 6 \mathrm{~g} / \mathrm{L}$ glucose, pH 7.4 with $\mathrm{NaOH}$ (50 $\mu \mathrm{M}$ d-AP5 was added)) using a gravity flow in-house built perfusion head. Neurons were stimulated using a platinum-iridium electrode at $10 \mathrm{~Hz}$ with $1 \mathrm{~ms}$ pulses. Tyrode buffer was modified with MES ( $\mathrm{pH} 5.5$ ) instead of HEPES for acid quenching of surface fluorescence. The alkalization of pHluorin-tagged vesicles was done by substituting Tyrode buffer with $50 \mathrm{mM} \mathrm{NH}_{4} \mathrm{Cl}$ and $69 \mathrm{mM} \mathrm{NaCl}$. Image analysis was done using ImageJ. 30-40 ROIs were marked in each repetition and the intensity change over time during the various treatments was 
measured. The fluorescence at any given time point was normalized to $F_{0}$ (average of first 10 frames). Time constants of recovery were measured by fitting the fluorescence decay after stimulation with a single exponential function. For measuring exocytic time constants, neurons were incubated with $5 \mu \mathrm{M}$ bafilomycin $\mathrm{A} 1$ in the bath for 2 minutes before proceeding with the stimulation.

\section{Single synaptic vesicle functional imaging}

Synaptic vesicles were isolated by immuno-isolation technique from cortical neurons at days in vitro (DIV) $15-17$ as detailed in supplementary data. The eluted SVs were immobilized on coverslips and used for functional imaging as described in Farsi et al., 2016. Briefly, the immobilized vesicles were labeled with $100 \mathrm{nM} \mathrm{VF2.1.Cl} \mathrm{for} 20$ minutes at room temperature. After incubating with the dye, the coverslips were washed to remove excess dye before proceeding with imaging with a TIRF microscope (Zeiss Laser TIRF 3). The vesicles were imaged on $100 \mathrm{X}$ objective (numerical aperture 1.45 ) at $1 \mathrm{~Hz}$ for 60 seconds. After acquiring the baseline measurement for $10 \mathrm{~s}$, same concentration of $\mathrm{Mg}^{2+}$-ATP was added to the coverslip and the fluorescence changes were recorded over time and corrected for photo-bleaching of the fluorophore over the same time scale before calculating membrane potential.

\section{Image analysis}

ImageJ was used for 2D and time series analysis as well as image processing for presentation. Imaris 8.0.2 software was used for 3D image analysis. Neuronal processes were marked with MAP2 staining channel with 'Surface' function in Imaris 8.0.2 and masked in the other channels (e.g. Bassoon, Rab3) to measure the intensity and number of synapses using 'Spot' function. The parameters of the algorithm were kept the same between the genotypes in each experiment. The statistics was exported and computed in excel.

\section{Statistical analysis}

Unless otherwise specified, statistical analysis was done in Microsoft excel 2010 and GraphPad Prism version 6 . The data are usually represented as mean values and error bars signify the standard error of mean (unless specifically stated in the graph). Significant difference between mean values of conditions was represented by ${ }^{*},{ }^{* *},{ }^{* * *}$ for $p$-values less than $0.05,0.01,0.001$ respectively. Unless otherwise stated, statistical significance was measured by an unpaired t-test. The biological and technical replicate numbers are mentioned in the figure legends. 


\section{SUPPLEMENTAL DATA}

\section{Supplementary methods}

\section{Sodium dodecyl sulfate polyacrylamide gel electrophoresis (SDS-PAGE)}

Standard SDS-PAGE was used to check for protein levels. SDS-PAGE was carried out to separate denatured proteins based on electrophoretic mobility, which is a function of their polypeptide chain length. Sodium dodecyl sulfate, an anionic detergent was used to denature the proteins and impart negative charge to the linearized polypeptide chain. The BIO-RAD electrophoresis system was used to perform the separation using a homemade gel $(10 \%, \mathrm{pH} 8.8)$ or $4-15 \%$ gradient gels (Bio-Rad), depending on the protein analyzed by immunoblotting. For the homemade gels, $5 \%$ stacking gel $(\mathrm{pH} \mathrm{6.8)}$ was used to allow proteins of varying size to enter at the same rate and thereby improve the resolution of electrophoresis. Protein samples were prepared by mixing the lysates with $6 \mathrm{X}$ Laemmli buffer (4.16M SDS, $47 \mathrm{~mL}$ Glycerol, 0.9mM bromophenol blue, $12 \mathrm{~mL} 0.5 \mathrm{M}$ Tris pH6.8, $5 \mu \mathrm{L} \beta$-mercaptoethanol in $100 \mathrm{~mL} \mathrm{dH}_{2} \mathrm{O}$ ) and denatured for 5 minutes at $95{ }^{\circ} \mathrm{C}$. The samples were allowed to cool down to room temperature and $40-75 \mu \mathrm{g}$ was loaded to each well in the gel. Electrophoresis was carried out at $80 \mathrm{~V}$ until the samples entered the separating gel, and then the voltage was increased to $110 \mathrm{~V}$ in the presence of $1 \mathrm{X}$ running buffer (12mM Tris base, $96 \mathrm{mM}$ glycine, $5 \mathrm{~mL} 10 \% \mathrm{SDS}$ in $1000 \mathrm{~mL} \mathrm{dH}_{2} \mathrm{O}$ ).

\section{Immunoblotting}

After electrophoresis, the proteins were transferred onto a nitrocellulose membrane using the BIO-RAD transfer system filled with $1 \mathrm{X}$ transfer buffer (25 mM Tris-Base, $0.2 \mathrm{M}$ Glycine, 20\% $(\mathrm{v} / \mathrm{v})$ Methanol in $\left.\mathrm{dH}_{2} \mathrm{O}\right)$. The transfer was done at a constant voltage $(25 \mathrm{~V})$ overnight at $4{ }^{\circ} \mathrm{C}$ or $100 \mathrm{~V}$ for 90 minutes at room temperature. The membrane was blocked in $5 \%$ milk prepared in 1X Tris-Buffered Saline and 0.1\% Tween 20 (TBS-T) at room temperature for 1 hour. It was followed by overnight incubation with primary antibody diluted in milk according to manufacturer's recommendation. The membrane was washed well to remove any non-specific bound antibody ( 3 short washes followed by $3 X$ wash for 5 minutes). Afterwards, the membrane was incubated with secondary antibody (Li-Cor), diluted 1:10000 for 1 hour at room temperature, followed by washing steps similar to the primary antibody treatment. The bands were then detected using the 
Odyssey infrared imaging system (Li-Cor). The band intensity was normalized to loading control or compared to experimental control when different cell/tissue types samples were compared.

\section{Immuno-isolation of synaptic vesicles from cultured neurons}

Synaptic vesicles were isolated from days in vitro (DIV) 15-17 cultured cortical neurons. The buffers used for isolation were prepared in advance and the $\mathrm{pH}$ of the solutions was set after they cooled down to $4^{\circ} \mathrm{C}$. Neurons were scraped in homogenization buffer (Sucrose $320 \mathrm{mM}, 5 \mathrm{mM}$ HEPES ( $\mathrm{pH} 7.4)$ ) and sample were centrifuged at $2000 \mathrm{rpm}$ for 10 minutes to collect the material. The pellet was re-suspended in $1 \mathrm{~mL}$ homogenization buffer containing $200 \mu \mathrm{M}$ PMSF and $1 \mu \mathrm{g} / \mathrm{ml}$ Pepstatin A. Cells were further homogenized by passing the sample with an $1 \mathrm{~mL}$ syringes through a cell cracker (10 up and down cycles) and the cell debris was removed by spinning down at 2000 rpm for 10 minutes at $4^{\circ} \mathrm{C}$. The supernatant was ultra-centrifuged at $80000 \mathrm{rpm}$ for $1 \mathrm{~h}$ (S140 AT rotor). The pellet was re-suspended in $200 \mu \mathrm{L}$ potassium gluconate buffer, homogenized at 900 rpm (10 strokes) and further passed through a 27 gauge syringe. Protein concentration of this sample was measured using Pierce BCA protein assay kit before proceeding with the immunoisolation.

$500 \mu \mathrm{g}$ of protein was incubated with VAMP2 monoclonal antibody at a ratio of 100:1 (protein: antibody ratio) in the presence of $0.5 \%$ (w/v) BSA in ice-cold potassium gluconate buffer overnight at $4^{\circ} \mathrm{C}$. The mixture was then incubated with protein G-coupled magnetic beads (Invitrogen) in the ratio 10:1 (beads: protein), which were washed 3 times before adding the protein/antibody complex. Subsequently, the beads were washed another 3 times, with intermittent vortexing and resuspended in suitable buffer depending on the next analysis. For single vesicle functional assays, the vesicles were eluted from the beads using VAMP2 control peptide in potassium gluconate buffer (Synthetic peptide corresponding to AA 2 to 17 from rat Synaptobrevin2, 104-2P SySy) at antibody: peptide ratio 1:5-1:10 for 1 hour (tubes with the mixture we intermittently tapped, in 3 steps of 15 minutes each followed by finally pooling the eluate together). The eluted SVs were immobilized on coverslips and used for functional imaging.

For the bulk acidification assay, the SVs were eluted in potassium gluconate buffer containing $100 \mathrm{mM}$ Choline-chloride and $10 \mu \mathrm{M}$ acridine orange in a quartz cuvette and acidification assay was performed using Fluolog III spectrofluorometer. The excitation and emission wavelengths 
were set at $491 \mathrm{~nm}$ and 530 $\mathrm{nm}$ according to the spectral characteristics of acridine orange. $\mathrm{Mg}^{2+}$ ATP was added to induce acidification of the SVs and the fluorescence change was recorded.

\section{Immobilization of vesicles on coverslips for functional assay}

Glass coverslips were cleaned by bath sonication in 2\% (v/v) Hellamanex -Il solution followed by sonication in filtered $\mathrm{ddH}_{2} \mathrm{O}$ for another 20 minutes. The coverslips were kept in $70 \%$ ethanol (filtered) until further use. The coverslips were rinsed in filtered water before using for vesicle imaging and then mounted in custom-built imaging chambers. They were then coated with $0.1 \%$ $(\mathrm{w} / \mathrm{v})$ poly-L-lysine(PLL) for 20 minutes and washed 3 times with filtered water and finally with assay buffer. Calculated quantity of SVs were diluted in assay buffer and incubated on PLL coated coverslips for 1 hour at $4^{\circ} \mathrm{C}$. After this, the non-adsorbed vesicles were washed away before proceeding with the functional assay.

\section{Lentivirus production}

293FT cells were plated at $10^{7}$ per $10 \mathrm{~cm}$ dish. The cells were transfected with lentivirus transfer plasmid containing cDNA along with envelop and packaging plasmids (3rd generation lentivirus system) using lipofectamine 2000, following manufacturer's protocol. The cells were maintained in S2 bio-safety lab henceforth and the medium was exchanged $14 \mathrm{~h}$ post transfection. The medium containing lentivirus suspension was collected, centrifuged at $3000 \mathrm{rpm}$ for 20 minutes to get rid of cell debris and concentrated. The concentrated particles were diluted in TBS $(\mathrm{pH} \mathrm{7.4)}$ and stored in $\quad-80^{\circ} \mathrm{C}$ until further use. The efficiency of the lentivirus was estimated by western blot and /or imaging when there was a fluorescent reporter.

\section{Primary neuronal cell culture}

Hippocampal and cortical primary neuron cultures were prepared from wild-type (WT) C57BL/6J or DMXL2 $2^{\text {loxp/loxp }}$ P0/P1 mice brains. The enzyme solution (in HBSS medium, $1.5 \mathrm{mM}$ cysteine, 20 $\mathrm{U} / \mathrm{mL}$ papain, $0.75 \mathrm{mM}$ EDTA, $1.5 \mathrm{mM} \mathrm{CaCl}_{2}$ and $10 \mu \mathrm{l} / \mathrm{ml}$ DNase), was prepared approximately 
30 minutes before dissection and incubated at room temperature (RT) to activate the papain. The dissection was performed under a laminar hood to maintain sterile condition.

The sterilized mice were decapitated and the hippocampus/cortex was isolated. After dissection, the tissue was digested using 2.5-3 mL of enzyme solution for $20-30$ minutes at $37^{\circ} \mathrm{C}$ on a shaker. The digestion was arrested by removing the enzyme and incubating with $10 \mathrm{~mL}$ of plating medium (Neurobasal medium with 5\% fetal bovine serum, 1\% glutamax, $2 \%$ supplement B27, 0.5\% penicillin/streptomycin) for 5 minutes. The serum in the plating medium helps to arrest the enzyme reaction. Further, the neuronal tissue was washed twice in $10 \mathrm{~mL}$ HBSS medium to remove any residual DNase. The tissue was gently triturated and plated on a poly-D-Lysine coated coverslip in a 12 well plate or $10 \mathrm{~cm}$ dish filled with pre-warmed plating medium. 75,000 - 150,000 cells were plated per coverslip depending on the viability and experimental requirement. The cells were maintained in a humidified incubator at $37^{\circ} \mathrm{C}$ with $5 \% \mathrm{CO}_{2}$. The plating medium was exchanged for neuronal medium (Neurobasal medium with 1\% glutamax, 2\% supplement B27, 0.5\% penicillin/streptomycin) after 12-16 hours. Sterile $\mathrm{dH}_{2} \mathrm{O}$ was used to fill the space in between the wells to maintain humidity and avoid unequal evaporation of medium from the wells. The neurons were used between DIV 14-20 for all the experiments.

\section{Transfection of primary neurons and cultured cells}

Plasmids were introduced into the primary neuronal cell and non-neuronal cells by electroporation (Amaxa TM 4D-Nucleofector X Unit, Lonza, Switzerland) or Lipofectamine 2000 (Invitrogen), by following the manufacturer's instructions. For electroporation, neurons were re-suspended as described previously in primary neuronal culture preparation and the cells were pelleted by centrifugation at $1000 \mathrm{xg}$ for 5 minutes. The supernatant was carefully removed and the cells were re-suspended in $100 \mu \mathrm{L}$ supplemented 4D-Nucleofector TM solution (Lonza). Calculated amount of plasmid DNA was added, and the mixture was transferred to the Nucleocuvette. Electroporation was performed with the $X$ unit of the Amaxa Nucleofector (Lonza) and the cells transferred to $2 \mathrm{~mL}$ of pre-warmed HBSS in a $5 \mathrm{~mL}$ tube and plated subsequently. 


\section{Microscopy}

\section{Immunofluorescence analysis by confocal microscopy}

Zeiss laser scanning confocal microscope (LSM800) with Airyscan, fitted with an inverted stand Axio Observer 7 was used for image acquisition from fixed samples. Plan Apo-Chromat DIC 63x/1,4 Oil and Plan APO 100x /1,4 OIL DIC M27 $\infty / 0,17$ objectives were used, and the sample was illuminated by transmitted light (Halogen) with manual differential interference contrast (DIC), fluorescence (HBO 100) and diode lasers (405, 488, 561 and 647nm). Cascadable non descanned detectors (NDD) with photo multiplier tube (PMT) were used for detection of 3 to 4 fluorescence channels simultaneously across the complete wavelength range. Microscope was operated by ZEN software (blue version, Zeiss) and post-acquisition image analysis and processing was done using ZEN black and ImageJ software.

\section{Live imaging by spinning disk confocal microscopy}

Spinning disc confocal microscopy was performed using the PerkinElmer UltraVIEW VoX system (Perkin Elmer, Waltham, Massachusetts, USA) with a spinning disk confocal scan head (CSUX1, Yokogawa) and a Nikon Eclipse Ti-E microscope with a, Plan APO $\wedge$ 60x / 1.40 Oil DIC N2 $\infty$ / 0,17 WD 0.13 OFN 25 and APO TIRF 100x / 1.49 DIC N2 $\infty$ / 0,13-0,20 WD 0.12 objectives, operated by Volocity (Improvision) software. Images were obtained using an EMCCD iXon 897 (Andor Technologies) camera. The samples were illuminated using a diode pumped solid state (DPSS) laser $488 \mathrm{~nm} / 50 \mathrm{~mW}$ diode and cobalt solid state $561 \mathrm{~nm} / 50 \mathrm{~mW}$ diode laser. Nikon eye piece filters (DAPI (Em: 450nm); GFP (Em: 520nm); TxRed (630nm)) were used for inspection with fluorescent light. 405/488/561/640 dichroic mirror was used for acquisition of images using multi-color channels. Cells were imaged at 23-37 ${ }^{\circ} \mathrm{C}$ temperature, depending on the experiment. Imaging buffer(136 mM NaCl, $2.5 \mathrm{mM} \mathrm{KCL}, 2 \mathrm{mM} \mathrm{CaCl}_{2}, 1 \mathrm{mM} \mathrm{MgCl}_{2}, 10 \mathrm{mM} \mathrm{HEPES}$, in dH $\mathrm{d}_{2} \mathrm{O}$, $\mathrm{pH} 7.4,300 \mathrm{mOsM} / \mathrm{kg}$ ) was used for live imaging of fibroblasts.

\section{Total internal reflection fluorescence (TIRF) microscope}

TIRF was used to image the isolated synaptic vesicles for functional analysis. Carl Zeiss motorized Laser TIRF 3 integrated in the AxioObserver research platform, and operated by ZEN blue software was used for used for this purpose. $488 \mathrm{~nm}(100 \mathrm{~mW} / 20 \mathrm{~mW})$ laser guided through a broad band fiber into the microscope and a single pass GFP filter facilitated the optimal 
illumination of samples. aPlan-Apochromatic 100x/1.46 Oil objective was used. The motorized zFocus of AxioObserver Z1 allowed for fine focusing. An EM CCD camera quant EM from Roper scientific with $13 \mu \mathrm{m}$ pixel size and a $512 \times 512$ chip enables fast detection and is integrated completely in AxioVision. 


\section{Supplemental figures}

A

Rbcn-3a-EGFP
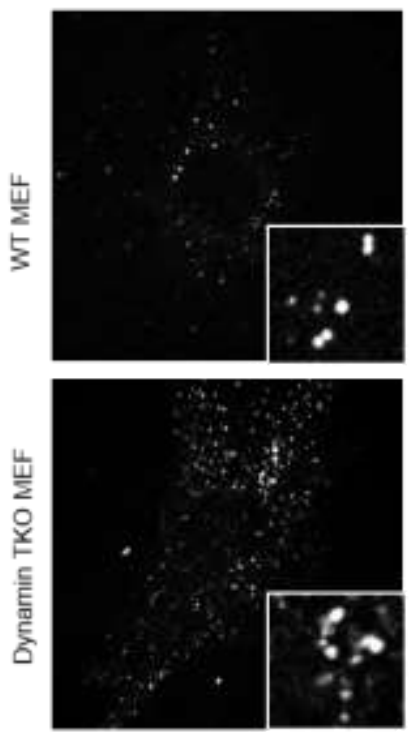

B

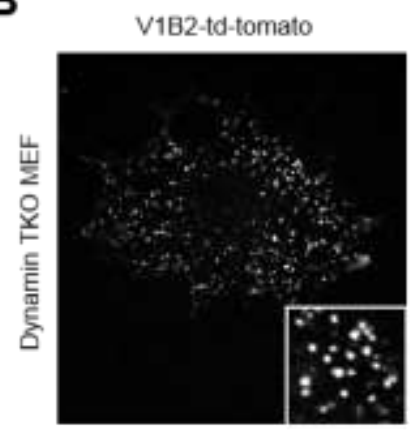

C

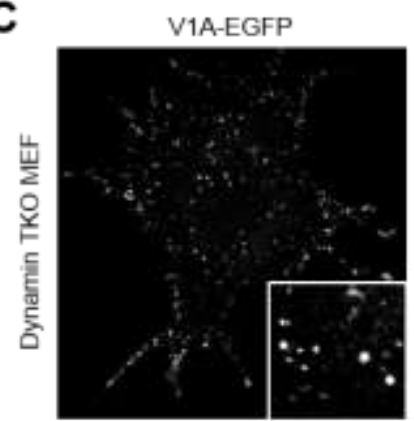

mRFP-o2
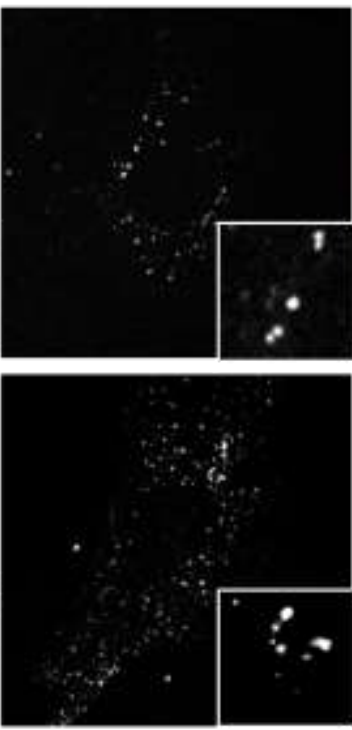

12-EGFP

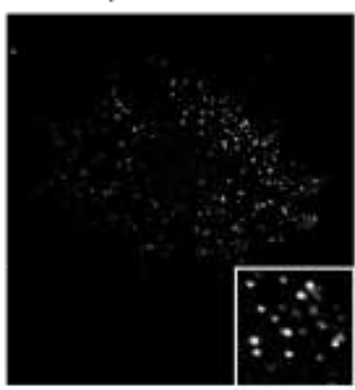

mRFP-CLC

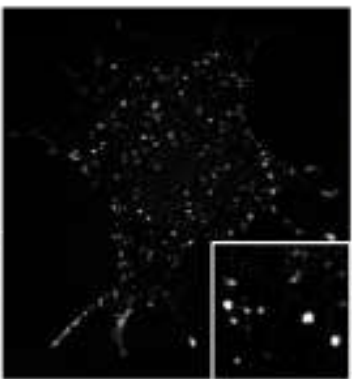

overlay
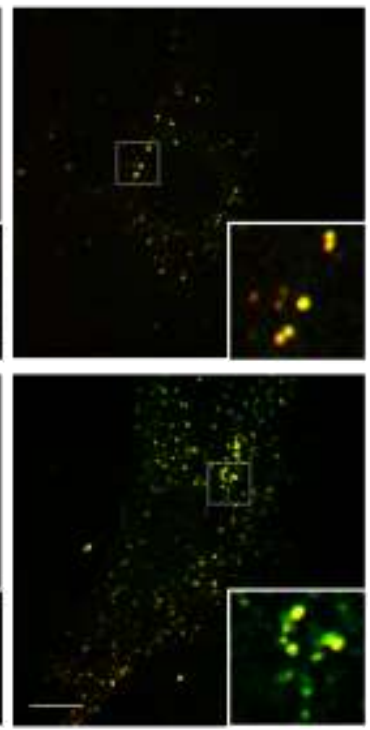

overlay

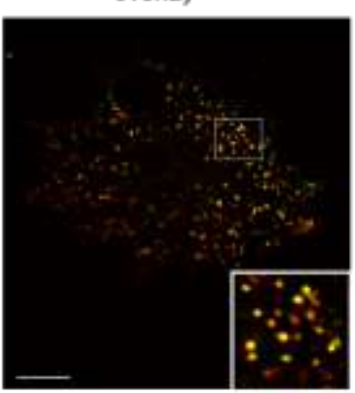

overlay

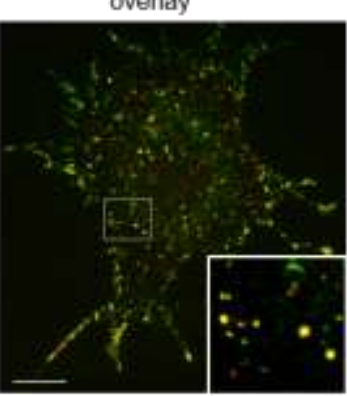

Figure S1. Rbcn-3a and v-ATPase are localized to clathrin coated pits (CCP) in fibroblasts 
(A) Rbcn-3a signal overlapped with $\sigma$ subunit of AP2 adaptor complex in control fibroblasts. In the Dynamin TKO MEFs with arrested CCPs, numerous puncta with Rbcn-3a signal overlapping with $\sigma$ subunit was observed.

(B) V-ATPase complex subunit V1B in the Dynamin TKO MEFs was found on the arrested CCPs, together with $\mu 2$ subunit of AP2 complex.

(C) V1A subunit of the V-ATPase complex is also found together with C-LC at the CCPs.

Scale bar $10 \mu \mathrm{m}$. (3 independent repetitions with at least 30 cells per condition).

A

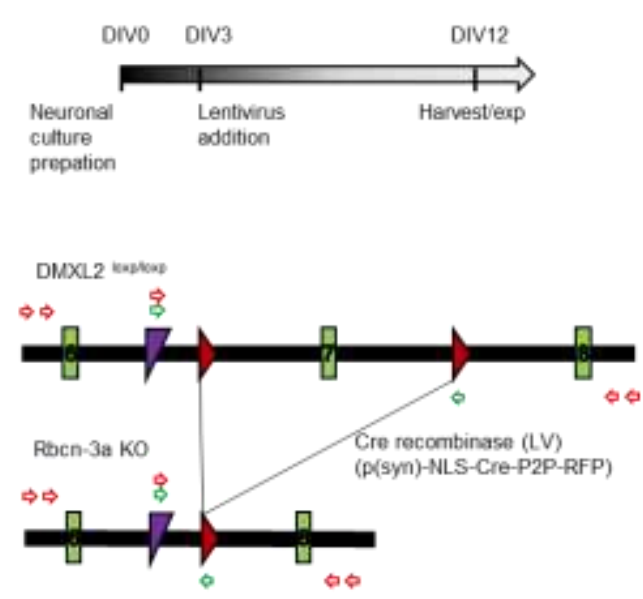

B
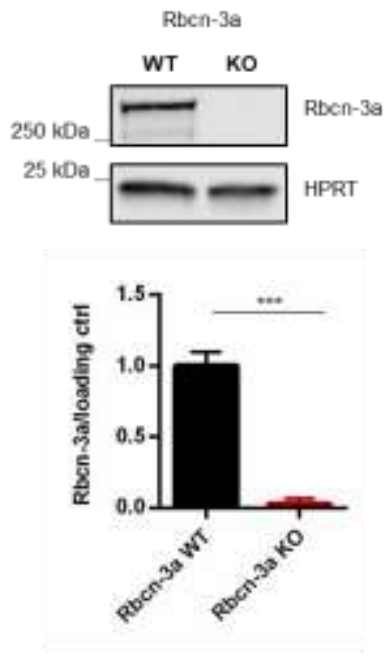

C

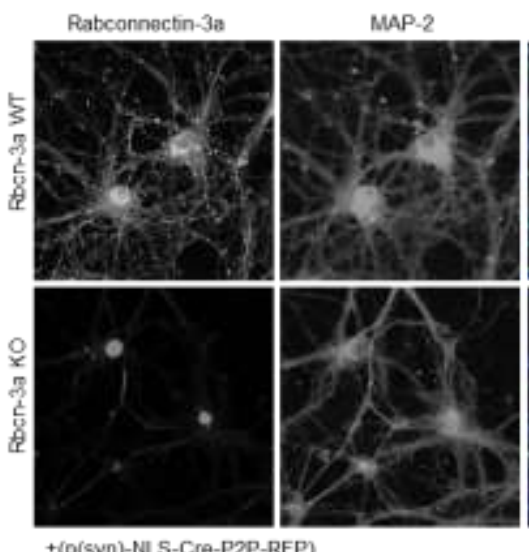

D
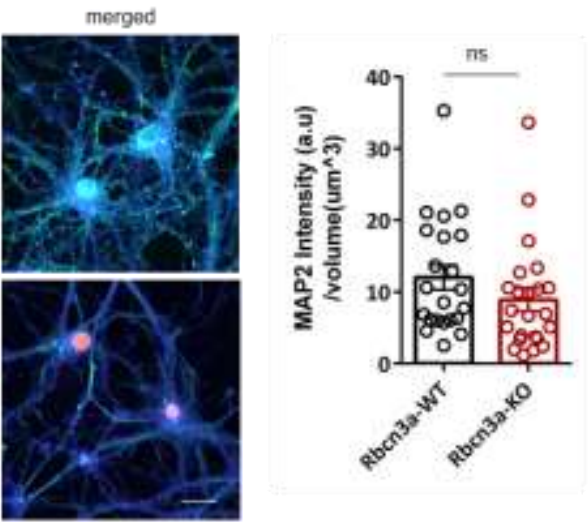

Figure S2. Rbcn-3a KO strategy and validation in neurons

(A) Strategy to obtain KO of Rbcn-3a in neuronal culture from DMXL2 loxp/loxp mice using lentiviral mediated cre recombination. The expression of Cre ( $p($ Syn)NLS-Cre-P2P-RFP) was driven by a synapsin promoter and RFP targeted to the nucleus was used a marker to select $\mathrm{KO}$ neurons.

(B) Representative Western blots to indicate the efficient removal of Rbcn-3a from neurons. HPRT was used as a loading control.

(C) Immunofluorescence in Rbcn-3a WT and KO neuron against Rbcn-3a and MAP2 shows that $\mathrm{KO}$ is effective and the synaptic signal for Rbcn-3 is not present anymore. The KO neurons were identified by the presence of RFP in the nucleus. Scale bar $15 \mu \mathrm{m}$.

(D) The intensity of MAP2 signal in the volume of the processes in Rbcn-3a KO neurons was not altered compared to the controls. (3 experiments, 30 cells per condition). 
A

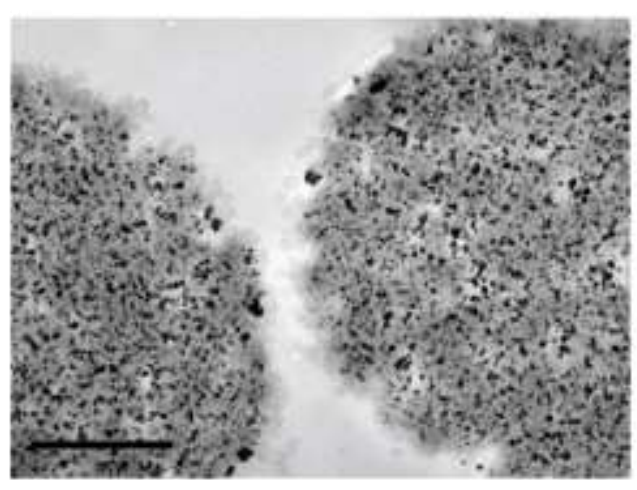

C

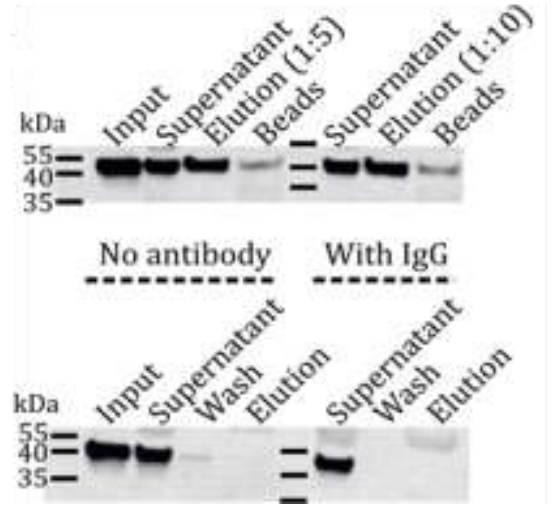

B

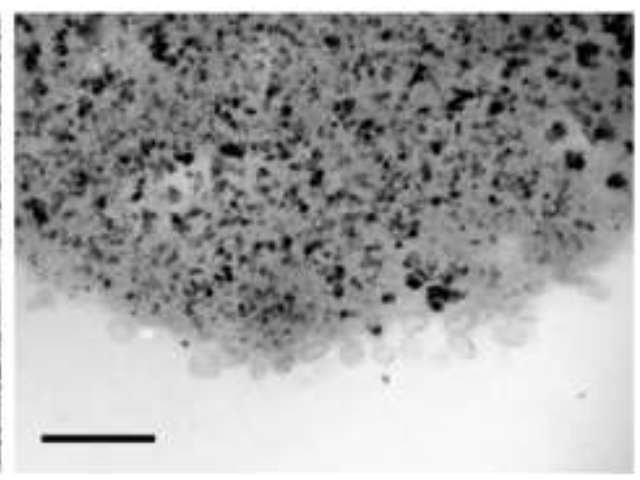

D

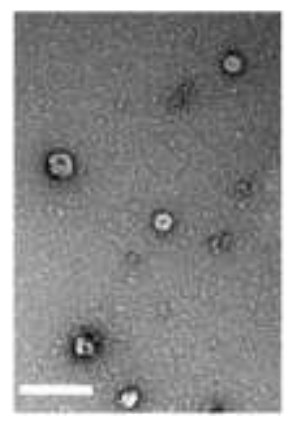

E

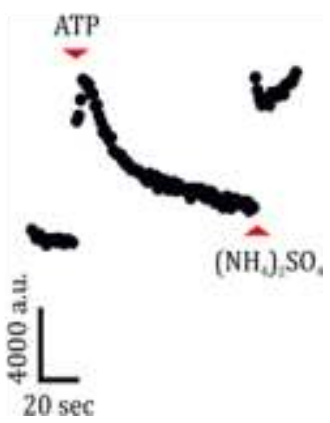

\section{Figure S3. Synaptic vesicles isolated by immuno-isolation were functionally active}

(A) EM image of magnetic beads after incubation with the neuronal cell suspension containing SVs. Scale bar 500nm.

(B) Non-specific binding of large organelles was not detected on the beads. Scale bar: $200 \mathrm{~nm}$.

(C) WB on the immuno-isolation samples against synaptophysin. Blot in the top panel shows the enrichment of synaptophysin signal in the eluted sample fraction. (Input: neuronal homogenate, supernatant: Sample before eluting with the peptide and the beads: sample after elution, boiled with loading buffer). Non-specific binding of SVs to the beads (no antibody) and to the antibody (with IgG) was not detected, as seen in the bottom blot. In both the control reactions, the material remained in the supernatant.

(D) Electron microscopy image confirmed the presence of SVs in the eluted sample. Scale bar: $200 \mathrm{~nm}$.

(E) The changes in emission intensity of acridine orange, which was used as a $\mathrm{pH}$ sensitive probe to label isolated SVs. The vesicles were functional: upon adding $1.8 \mathrm{mM} \mathrm{Mg}^{2+}$-ATP, the fluorescence of the sample decreased dramatically and this was recovered by adding $50 \mathrm{mM}$ ammonium sulfate $\left(\mathrm{NH}_{4}\right)_{2} \mathrm{SO}_{4}$, which induces alkalization of the vesicle lumen. 


\section{Discussion}

SV recycling include a sequence of regulated processes like exocytosis (fusion process), endocytosis (fission process), SV acidification, NT refilling and maturation of SV into a fusioncompetent vesicle. It is attributed as one of the finely controlled process and many key players involved have been characterized. Particular steps like exocytosis and endocytosis have been extensively researched in the last 30-40 years. The intermediate steps in the SV recycling process like coupling of exo- and endocytosis, how and when the vesicles proceed with acidification, refilling and become fusion-competent after endocytic reformation are not yet fully understood. The goal of my thesis was to address some of the open questions in SV recycling process.

The small size of SVs, the high speed of recycling process and the transient nature of the recycling intermediates pose challenges to decipher the molecular mechanism of SV cycle completely. The steps in SV recycling have been investigated in various model organisms, using a plethora of techniques depending on the spatial and temporal resolution needed to address the particular question. Cultured neurons as a model system are a good starting point to understand basic presynaptic mechanisms and address the function of molecular player in quest. However, the presence of local recycling makes it difficult to uncouple exocytic effect from endocytosis. In this case, neurosecretory adrenal chromaffin cells are useful, since they contain LDCV which undergo regulated fusion and the process of endocytosis involves recycling of the retrieved proteins and membrane to near Golgi area, to be reused for the next cycle of events. The existence of local recycling step is not well established in chromaffin cells. Hence, I used the neurosecretory cells from endophilin mice mutants (mice described in (Milosevic et al., 2011)) to answer the question whether endocytic proteins, endophilins $A$ have a direct role in vesicle exocytosis. In the next step, to uncover a potential link between endocytosis, acidification and SV maturation process, I focused on the role of Rbcn-3a, a putative V-ATPase regulator in SV recycling process. Since the genetic ablation of v-ATPase itself or its regulator is embryonically lethal, I used conditional mice (described in (Tata et al., 2014)) and induced KO in culture (using Cre lentivirus as reported in (Vardar et al., 2016)) to characterize the function of Rbcn-3a in SV recycling. I used a combination of physiological, morphological as well as biochemical techniques to address these two questions, and the main results are listed below. 
The first study explored a potential direct role for endophilin in exocytosis using the adrenal chromaffin cell model system. We found an unanticipated direct role for endophilin in LDCV mobilization, priming and fusion, independent of its role in endocytosis. The lack of endophilin altered the LDCV distribution in the cell, but did not affect the LDCV number or protein machinery for fusion and fission processes. The effect in exocytosis was mediated through its $\mathrm{SH} 3$ domain, at least in part through its interaction with intersectin-1.

In the second study, the link between endocytosis, acidification and fusion-competent SV formation was investigated with a focus on a novel v-ATPase regulator called Rbcn-3a. Rbcn-3a is a large $340 \mathrm{kDa}$ protein with unknown function at the synapse. I found it to be present on many organelles that acidify; including SVs and CCVs. Loss of Rbcn-3a in neurons in culture resulted in impaired synaptic activity, altered SV recycling and defective acidification of vesicles. Rab3 recruitment to SVs and their stability on the vesicles was affected due to the absence of Rbcn3a. Additionally, endosome-like and lysosome-like-structures accumulated in the neurons without Rbcn-3a. This study presented a novel link between several key steps in the vesicle life cycle and cellular trafficking pathways.

These two studies addressed some questions related to the missing links in SV recycling process. The main findings and their implications are discussed in the following sections in detail.

\subsection{Role of endophilin in exocytosis}

Several studies pointed to role(s) for endophilin in processes beyond endocytosis at the synapse. Curiously, the lack of endophilin protein in Drosophila, or all three endophilins in mice, resulted in impaired SV recycling process, with accumulation of CCVs at the synapse. Synaptic transmission was compromised in the KOs, but was not completely abolished (Milosevic et al., 2011; Verstreken et al., 2003). It was difficult to decipher if this was due to an indirect effect of defective endocytosis, or a possible direct role for endophilin in vesicle exocytosis, or both. To address this question, we used neurosecretory model, adrenal chromaffin cell in culture from endophilin mice mutants. Chromaffin cells are well established model to study regulated exocytosis (briefed in section 1.6.1, aims and experimental model). A fast local recycling pathway has not been reported in chromaffin cells, so they present a good system to dissect the role in exocytosis from 
endocytosis. They further allow precise spatial and temporal regulation of vesicle release, so we employed this neurosecretory model system to understand the function of endophilin in the fusion process. Fast capacitance and amperometry recordings were used to check for a role for endophilin in LDCV fusion and the morphological correlate of the findings were verified using classical EM analysis and confocal microscopy. The study showed that endophilin A1 and A2 have a role in vesicle exocytosis, independently of their function in endocytosis.

\subsubsection{Endophilin A has a direct role in vesicle exocytosis}

The study revealed that endophilin $\mathrm{A} 1$ and $\mathrm{A} 2$ have a role in vesicle exocytosis, independently of their function in endocytosis. Flash experiments, which stimulated chromaffin cells by uncaging calcium, showed that lack of endophilin impairs LDCV exocytosis by affecting vesicle mobilization, priming and fusion. Analysis of the different release components showed that the RRP, SRP and the sustained pool were reduced in the absence of endophilin. This could be due to an alteration in the number of LDCVs available for release, or, an unknown function for endophilin in exocytosis process itself. Removal of endophilin from Drosophila or mice neurons resulted in a reduction of vesicle number due to defective recycling (Verstreken, et al., 2002, Milosevic et al., 2011). Studies of other BAR domain-containing proteins, like Protein Interacting with C Kinase 1 (PICK1), in chromaffin cells revealed a role in LDCV biogenesis (Pinheiro et al., 2014). Surprisingly, the lack of endophilin did not affect the overall number of LDCV in the cell or per cell area, as revealed by both ultrastructural and light microscopy experiments. Although the number of vesicles was not altered, their distribution in the proximity of PM was changed in endophilin TKO chromaffin cells. This suggested a role for endophilin in vesicle recruitment, through yet unknown mechanism, possibly linked to actin and intersectins. Further, the defects in exocytosis could be rescued by expressing endophilin A1 or A2 alone in TKO cells. This suggested an overlapping role for the different endophilins in exocytosis in chromaffin cells.

\subsubsection{Endophilin's role in exocytosis is endocytosis independent}

Endophilin is a well-studied endocytic protein, known to be involved in various membrane and protein retrieval pathways (Milosevic, 2018). Two major recycling pathways were identified in chromaffin cells: a fast recycling pathway, dependent on dynamin and GTP, and a slow endocytic pathway, which is clathrin and dynamin dependent (Artalejo et al., 1995, 2002; Smith and Neher, 
1997). The various endocytic pathways tested at 8-10 minutes were not majorly affected in the endophilin TKO chromaffin cells: the recycling of proteins (e.g. transferrin) and membranes (fluorescently-labelled membrane probe mCling (Revelo et al., 2014). In the case of $\mathrm{mCling}$, it is possible that the kinetics of endocytosis is delayed in the first minutes of the process in the absence of endophilin, but this remains to be tested in future.

LDCV generation/maturation steps takes several minutes to hours, thus the initial endocytic delay may not be relevant for the observed effects in exocytosis directly. However, endophilin can inflict an indirect effect on the LDCV composition and/or membrane and protein recycling processes in chromaffin cells. Additionally, perturbation of endocytic process was postulated to affect vesicle release by occluding the release site and re-priming of vesicle (Kawasaki et al., 2000). Although the process of endocytosis is relatively slow in comparison to the exocytic process, the effect on exocytosis developed much faster when components of endocytic mechanism was perturbed (Hosoi et al., 2009). It is possible that the lack of endophilin affects this intermediate step of site clearance between exo- and endocytosis, wherein the vesicles are available, but are not able to fuse to the PM upon stimulation. To explore these possibilities, we checked for the levels and distribution of several key exocytic factors as well as endocytic proteins. In short, none of the tested exocytosis factors (SNAREs, synaptotagmin-1, Muncs) as well as key endocytic proteins dynamins, AP2 and AP180 was noticeably changed in endophilin TKO cells. Further, analysis of CgA-positive LDCVs for levels of an exemplary protein synaptotagmin-1 showed no alterations in the TKOs compared to controls. Taken together, endophilin loss did not change the level or distribution of key exocytic factors, suggesting that the effect is probably direct and not due to alteration in LDCV composition.

\subsubsection{Mechanism of endophilin's action in exocytosis: BAR domain vs SH3 domain}

Two models have been proposed to elucidate function of endophilin in endocytosis, namely the scaffolding model and membrane bending model (Bai et al., 2010). In the scaffolding model, endophilin mediates its role through protein-protein interaction by $\mathrm{SH} 3$ domain. For example, it is involved in recruiting dynamin for membrane fission and synaptojanin-1 to facilitate uncoating of coated vesicles in endocytosis (Milosevic et al., 2011; Ringstad et al., 1997; Schuske et al., 2003; Verstreken et al., 2003). Alternatively, according to the membrane bending model, BAR domain 
of endophilin participates in membrane bending process and influence endocytosis. Endophilin can undergo homo-/hetero-dimerization and promote positive membrane curvature. The dimers form a concave membrane-binding surface. Hence they act as membrane curvature sensors as well as participate in curvature generation (Bai et al., 2010; Farsad et al., 2001; Gallop et al., 2006; Itoh et al., 2005; Masuda et al., 2006). All these studies showed that both the BAR domain as well as the $\mathrm{SH} 3$ domain is important for endocytic functions. To understand which domain of endophilin is important for exocytosis, we performed the rescue experiments with BAR domain only constructs. Surprisingly, the release was further reduced, suggesting that there is a dominant negative effect and indicated that endophilin's SH3 domain is needed for exocytosis. Many proteins participating in SV recycling (e.g. dynamin, synaptojanin, intersectin, VGLUT1) interact with endophilin through its $\mathrm{SH} 3$ domain as discussed earlier. Among the known interactions, dynamin and intersectin have been implicated in LDCV exocytosis process and we explored a potential mechanism of action through these interaction partners.

\subsubsection{Role of endophilin and dynamin in neurosecretory vesicle fusion}

In chromaffin cells, a dynamic fusion pore behavior was recently reported to regulate the cargo release and vesicle retrieval. This was shown to be controlled by a competition between expansion (mediated by F-actin dependent tension) and constriction (governed by calcium and dynamin) (Shin et al., 2018). A prevalent view is that fusion involves formation of a narrow pore that is closed rapidly, which determines the speed and extent of content released ('Kiss'-and'run'), or, alternatively the pore expands irreversibly and results in full collapse (Alabi and Tsien, 2012; Lindau and Alvarez de Toledo, 2003). Dynamin is known to form a collar-like structure at the neck of a clathrin-coated pit and support fission by its GTPase activity (Ferguson and De Camilli, 2012). Several observations on pore dynamics regulation by dynamin lead to a model wherein dynamin surrounds the fusion pore and participates in constriction, thereby regulating the content released (Anantharam et al., 2011; Fulop et al., 2008; Graham et al., 2002). It is not yet known how dynamin is recruited to the site of fusion to regulate the amount of content released by the vesicles. Some studies have suggested that the interaction of dynamin with SNARE proteins could be playing a role in this (Galas et al., 2000; Okamoto et al., 1999). On the other hand, dynamin is recruited to the CCPs, the site for its fission activity by endophilin and amphiphysin, BAR proteins that sense, bind and/or generate curved membranes (Ferguson and 
De Camilli, 2012). Since the time constants of release of RRP vesicle is altered in the endophilin TKO chromaffin cells, it is tempting to postulate that endophilin's impact on exocytosis is mediated through its interaction with dynamin and fusion pore dynamics. However, basic single spike amperometry analysis from the TKO chromaffin cells did not show changes in the foot properties, arguing against this hypothesis. Further thorough analysis should be done along this line to test if endophilin has a role in vesicular content released and fusion pore dynamics.

\subsubsection{Coupling mechanisms of exo- and endocytosis}

The process of how exocytosis is coupled to endocytosis is one of the open questions in SV recycling process and several mechanisms have been proposed. Calcium, membrane lipids composition and metabolism, interactions between SV proteins and endocytic protein machinery and active/periactive zone adaptors are some of the suggested links between the processes (Haucke et al., 2011; Maritzen and Haucke, 2018). Regulation of membrane lipid composition and metabolism during SV recycling could also be a potential mechanism to couple exocytosis with endocytosis (Di Paolo and De Camilli, 2006). Several proteins from the endocytic machinery can bind to membranes (e.g. adaptor proteins, BAR domain containing proteins). BAR proteins can enhance the curvature and function as molecular scaffold (Gallop et al., 2006). Through the membrane binding properties, endophilin could participate in the fusion process itself directly or through interaction with other proteins (say dynamin). It is not clear how vesicle exocytosis triggers endocytic retrieval process. The newly added SV proteins in the PM could act as a platform to recruit the endocytic factors to the site of endocytosis from the cytosol. Also, the membrane tension changes upon full fusion of vesicles and this could be sensed by BAR proteins (e.g. endophilin), which can then organize the site for endocytosis through their SH3 interaction hub. Although it is a tempting hypothesis to explain endophilin's role in exocytosis, we have to do further experiments along this line to conclude about the influence of lipids on endophilin's role in exocytosis.

Calcium is a core component required for regulated exocytosis as well as influence endocytic retrieval process (reviewed in (Neher and Sakaba, 2008)). Calmodulin, a calcium binding protein was suggested to be the major calcium sensor for endocytosis (Wu et al., 2009). Further, a 
phosphatase protein calcineurin, a downstream target of calmodulin, has been shown to dephosphorylate numerous endocytic proteins collectively termed as dephosphins (like dynamin, synaptojanin-1, AP180, among others) to facilitate endocytosis and retrieval process (Cousin and Robinson, 2001; Lee et al., 2005, 2004; Murthy and Camilli, 2003). Further analysis of the direct influence of calcium on endophilin's function in exocytosis is needed.

In the resting state, endocytic proteins were found to exist in a matrix along with vesicles in the terminal and mobilized towards the endocytic sites upon calcium induced dephosphorylation (Shupliakov, 2009). Interestingly, synapsin, a peripheral SV protein implicated in vesicle clustering was found at sites of endocytosis upon stimulation (Bloom et al., 2003; Tao-Cheng, 2006). The dynamics of synapsin re-clustering and SV re-clustering at the terminal was comparable (Li and Murthy, 2001). Further, synapsin interacts with several endocytic proteins, for example endophilin, amphiphysin and intersectin (Evergren et al., 2007; Onofri et al., 2000; Ringstad et al., 1997, 1999). Additionally, yet another peripheral SV protein Rab3 regulated SV exocytosis mediated delivery of endophilin to the site of endocytosis (Bai et al., 2010). Taking all these findings together, it seems that the protein-protein interaction between peripheral SV factors and endocytic machinery is important to couple exocytosis with retrieval mechanisms and these proteins have additional role in SV recycling apart from their primary described functions. Lack of endophilin could impact this protein-protein network and change the distribution of some of these factors during the recycling process. To check this possibility, we tested the levels and distribution of several endocytic proteins. Interestingly, the protein distribution of intersectin-1 in endophilin TKO chromaffin cells was altered (discussed in the next section) in resting condition. We further explored a possible mechanism to explain endophilin's role in exocytosis through its interaction with intersectin.

\subsubsection{Role of endophilin-intersectin interaction in LDCV exocytosis}

Intersectins (ITSN) are adaptor proteins with multiple SH3 domains that interacts with proteins form endocytic machinery (dynamin, amphiphysin, endophilin) as well exocytic SNARE protein (SNAP-25) and suggested as a molecular link between exo- and endocytosis (Pechstein et al., 2010; Sakaba et al., 2013). Actin cytoskeletal dynamics is another important process that 
regulates vesicle recycling. Intersectin-1 is a GEF for activating cdc42, which accelerates actin assembly (Hussain et al., 2001). Intersectin-1L was found at sites of exocytosis, activate cdc42 mediated stimulation of actin assembly at docking sites and thereby increases release in secretory cells (Malacombe et al., 2006).

Analysis of intersectin in control and endophilin mutant chromaffin cells under resting and stimulated conditions revealed a change in distribution and dynamics of these proteins. Intersectin-1 is generally localized in the cytosol during resting state, but translocate near PM upon stimulation in control chromaffin cells (Malacombe et al., 2006). In contrast, ITSN-1 was already enriched near the PM in the endophilin TKO cells and stimulation did not induce changes in the distribution. In an attempt to understand the molecular mechanism of action of endophilin in exocytosis, we tried to rescue the defects in endophilin TKO cells with endophilin that cannot bind intersectin-1 (endophilin 1-E329K+S366K, reported in (Pechstein et al., 2015)). The altered distribution of intersectin-1 could be rescued by expressing full-length endophilin $A 1$, but not by endophilin $A 1$ unable to bind intersectin-1 (endophilin 1-E329K+S366K). This suggests that endophilin acts as a repressor of intersectin by keeping it away from the PM and controls vesicle availability for fusion.

Taken together, our data suggests that endophilin regulates vesicle recruitment and exocytosis directly, at least partially through its interaction with intersectin and independent of its function in endocytosis. This newly found role for endophilin in exocytosis is particularly relevant in the context of exo-endocytosis coupling mechanisms.

\subsection{Rbcn-3a is a putative v-ATPase regulator that links endocytosis, acidification and SV maturation}

\subsubsection{Rbcn-3a is an evolutionarily conserved v-ATPase regulator}

Regulators of v-ATPase in various cellular processes are not well studied and only few works report on proteins or mechanism of its regulation. V-ATPase has evolved to perform a wide range of function, centered on its proton pumping activity. It has also been attributed to perform some non-canonical functions, apart from being an electrogenic pump (Maxson and Grinstein, 2014). 
Rbcn-3 is a putative v-ATPase regulator and its function is evolutionarily conserved from yeast to mammals. However, the cellular process affected as a result of acidification is different depending on the cell type, organelle at quest and complexity of the organism. One of the key questions of my thesis was to address the role of Rbcn-3a in the brain and specifically on SVs at synapses? Before elaborating on main findings, I have briefly compared its function across species in the context of v-ATPase activity regulation.

One of first reported mechanism of regulation of v-ATPase was assembly/disassembly cycle of the v-ATPase complex (Sumner et al., 1995). The re-assembly of the complete v-ATPase holoenzyme was mediated by RAVE (regulator of $\mathrm{H}^{+}$-ATPase of vacuolar and endosomal membrane) complex. This process also required phosphatidylinositol 3-kinase signaling (Seol et al., 2001; Smardon et al., 2002). The RAVE complex comprises of Rav1, Rav2 and Skp1 proteins. Rav1 is the yeast homolog of Rbcn-3a, which in complex with Rav2 regulates the glucose dependent assembly/disassembly in yeast. Although Rav2 is not found in mammals, Rbcn-3a exerts its function possibly through a complex formed with a different protein called WD-repeat 7 (referred as Rbcn-3b) (Kawabe et al., 2003). Alternatively, in follicle cells and imaginal disc cells in Drosophila, Rbcn-3a and $3 b$ homologs were identified to play a role in endocytic trafficking and impaired Notch signaling. The lack of Drosophila homologs resulted in the accumulation of Notch and other membrane proteins in the late endosome compartment (Sethi et al., 2010; Yan et al., 2009). Despite being in complex with different proteins, its v-ATPase regulator function seems to be preserved. The exact mechanism of how Rbcn-3 complex regulates v-ATPase activity is not yet known. It is not known if Rbcn-3a and $3 b$ complex is present on every organelle that acidifies in mammals and the localization as well as the site of action remains to be tested. In my thesis, I primarily focused on Rbcn-3a, characterized its cellular localization in the trafficking organelles and explored its function in SV recycling.

\subsubsection{Links between endocytosis and vesicle acidification}

In the SV cycle, electrochemical gradient generated by the v-ATPase drives NT refilling step and the role of the proton pump is studied in this context. During the fusion process, the integral SV proteins, including V-ATPase are added to the PM and retrieved by endocytic pathways. The 
stage at which acidification happens during/after the retrieval process from the PM or endocytic intermediate is not yet known. The proton pumps being functional throughout the SV recycling process may not be energetically favorable for the synapse, so question arises if the pump is active throughout the SV cycle or is actively regulated.

A pH-based sensor pHluorin, tagged to the luminal domain of SV proteins are a well-established tool to follow presynaptic activity in neurons (Miesenböck et al., 1998; Sankaranarayanan et al., 2000). During stimulation, the fluorescence observed is a balance between exocytosis and recovery (endocytosis and re-acidification) at all times. The use of v-ATPase blocker bafilomycin A1 prevents the re-acidification of a newly formed vesicle by endocytosis and helps to uncouple exocytosis from endocytosis. The study on WT neurons with bafilomycin A1 showed that the vesicles could be trapped in an alkaline state without affecting the kinetics of endocytosis (Sankaranarayanan and Ryan, 2000). The kinetics of recovery after stimulation in such assays reflects the combined rate of endocytosis and re-acidification (Atluri and Ryan, 2006; Leitz and Kavalali, 2011). Using a short acid pulse application following series of action potential in hippocampal neurons, the re-acidification kinetics was suggested to be $\sim 4-5$ s (Atluri and Ryan, 2006). Additionally, the time taken for endocytic retrieval was suggested to be $\sim 4 s$ (Leitz and Kavalali, 2011). Recently, SV proteins were shown to undergo endocytosis on multiple time scales at physiological temperature (Soykan et al., 2017). Adapting these strategies, pHluorins were used to study the recycling defects in endocytic mutants (Mani et al., 2007; Milosevic et al., 2011). However, the delayed post-stimulus recovery observed was suggested to be due to the delay in endocytosis, but the effect of re-acidification was not excluded. It was difficult to separate the two components of recovery in neurons of these mutants (or in general in cultured neurons) since uncoating was only delayed, but not blocked and we cannot distinguish if the fluorescence from an internalized endocytic intermediate exists because of an acidification problem, or defects in steps of endocytosis.

In an attempt to address the debate on whether endocytic coated vesicle undergoes acidification or not, CCVs were isolated from mice brain and $\Delta \mu_{H_{+}}$was characterized the single vesicle level by imaging. Previously establish single vesicle imaging technique was adapted (Farsi et al., 2016) to determine the membrane potential (using VF.Cl.2.1) and pH (using synaptopHluorin) of CCVs. The results of this recent work showed that the clathrin coat blocks the v-ATPase activity and can be a potential v-ATPase regulatory mechanism at the synapse (Farsi et al., 2018). Future 
experiments focused on performing imaging of single vesicles derived from mutant animals will be crucial to further understand the role of other endocytic factors in v-ATPase function and give clarity on whether clathrin coat alone is sufficient to block the pump, or other accessory factors are involved in this process, and when exactly the regulation by the coat is released in the endocytic pathway.

A fully functional v-ATPase with $\mathrm{V}_{0}$ and $\mathrm{V}_{1}$ subunits was detected in these isolated CCVs (Farsi et al., 2018). Interestingly, I found Rbcn-3a, the putative v-ATPase regulator on both isolated SV as well as CCV. Rbcn-3a was initially shown to be enriched in crude SV fraction from rat brains (Nagano et al., 2002). To check if Rbcn-3a is present only on coated vesicles, or also recruited to site of formation of the coated structure (CCP), I capitalized on mouse embryonic fibroblasts (MEFs) lacking the fission protein dynamin TKO (cells without all three dynamins). Rbcn-3a localized to the CCP in dynamin TKOs along with endocytic markers like clathrin-LC and sigma2 (subunit of AP2 adaptor complex). Additionally, v-ATPase also localized to CCPs, overlapping with above mentioned endocytic marker proteins. These results suggested that the v-ATPase and its regulator Rbcn-3a are present on forming endocytic vesicle and potentially the activity is kept under check by the clathrin coat. Clathrin coat mediated regulation of v-ATPAse and the presence of Rbcn-3a on CCVs and coated pits add new links between endocytosis and acidification process at the synapse.

\subsubsection{Building tools to study the role of Rbcn-3a at the synapse}

After the initial report on identification of Rbcn-3 complex, further studies were limited by the lack of tools and large size of the protein to characterize its function at the synapse (Kawabe et al., 2003; Nagano et al., 2002). In a search for novel regulators of v-ATPase, we cloned the full-length gene DMXL2 encoding for Rbcn-3a and characterized its expression pattern in the non-neuronal cell and synapse. A complete knock-out in mice was embryonically lethal (Tata et al., 2014). vATPase function is important for several basic life processes, thus Rbcn-3a loss could be detrimental. Besides, Rbcn-3a is involved in the Notch and Wnt receptor signaling pathways and has a developmental function (Tuttle et al., 2014; Yan et al., 2009). To by-pass this, I used DMXL2 floxed mice and induced KO in culture. For studying SV recycling, I used cultured cortical and 
hippocampal neurons and combination of physiology, morphological and biochemical techniques. Neurons survived in culture and formed synapses; however, detailed developmental characterization is pending to test for potential problems with dendritic branching, given its importance in developmental pathways in other model organisms (the Cre-recombinase virus was added between 2-5 days after culturing).

\subsubsection{Role of Rbcn-3a in SV recycling}

A reduction in total integral SV protein in Rbcn-3a suggested a problem with presynaptic pathways or vesicle proteins being marked for degradation. Rbcn-3a KO synapse had a reduced number of vesicles observed by EM analysis, suggesting that the reduced integral proteins correlated with the reduction in the number of SVs. A reduced number of SVs could be due to defective recycling and accumulation of intermediates at the synapse.

Presynaptic activity monitored using VGLUT-pHluorin showed delayed recovery after stimulation in the absence of Rbcn-3a. As discussed in the section before, this could be due to a defective endocytosis or an influence on vesicle re-acidification. Interestingly, the kinetics of recovery was similar to that of WT neurons with half maximal concentration of v-ATPase inhibitor bafilomycin A1 (Sankaranarayanan and Ryan, 2000). On the other hand, complete block of the v-ATPase $(5 \mu \mathrm{M})$ did not affect the endocytic retrieval rate as well as the amount of FM styryl dye internalized upon stimulation (Sankaranarayanan and Ryan, 2000). This was in line with the observation in Rbcn-3a KO neurons, where the rate of endocytosis and the amount of FM dye uptake was not altered. Also, the fraction of surface stranded VGLUT-pHluorin moieties was not significantly different in Rbcn-3a KO neurons, and the total protein levels of tested endocytic factors were comparable between the genotypes. All of this suggested that the observed difference in recovery could be an acidification problem, but not due to retrieval, and that the vesicles are trapped in an alkaline state.

Acidification kinetics from single vesicle imaging suggested that SVs acidify in less than a second, but the kinetics measured from cultured neurons appeared much slower using the same probe (Atluri and Ryan, 2006; Farsi et al., 2016). Thus, it is difficult to separate endocytosis from acidification in bulk experiments with pHluorin in culture. The two components of electrochemical 
gradient that govern NT filling can be measured at the single vesicle level precisely (Farsi et al., 2016), but this had to be adapted to Rbcn-3a cultured neuronal system. Measurement of $\Delta \mathrm{pH}$ using isolated vesicles will give a concrete idea about the defects in acidification caused by Rbcn3a KO; however, this was technically challenging and should be addressed in the future. $\Delta \Psi$ of isolated SVs measured from neuronal culture using potentiometric probe VF2.1.Cl (Farsi et al., 2016; Miller et al., 2012) was smaller in Rbcn-3a KO. Given the defects in recovery after stimulation, without change in endocytosis and reduced membrane potential in Rbcn-3a, we can conclude that the defects are due to defective acidification in Rbcn-3a KO neurons.

The electrochemical gradient influences the vesicular NT fill state and this can affect transmission process in acidification mutants. Spontaneous vesicle fusion analysis is commonly used to interpret about vesicle fill state, which is affected by the transporters as well as the v-ATPase, although this is an indirect readout and can be influenced by post-synaptic receptors. For example, the quantal release is reduced in neurons from VGLUT1-deficient mice (Wojcik et al., 2004). mEPSC and mIPSC measured in cultured neurons as well as slices treated with bafilomycin A1 presented a reduced amplitude and a drastically reduced frequency, showing that blocking v-ATPase can possibly alter the vesicular content (Zhou et al., 2000). Optogenetically controlled acidification studies showed that partially filled vesicles had a reduced amplitude and frequency compared to fully acidified vesicles. Additionally, reduced filling of vesicle lowered the vesicular release probability (Rost et al., 2015). Rbcn-3a KO neurons showed a reduction in membrane potential and concomitantly the mEPSC frequency and amplitude was also reduced. It would be important to test if the vesicular release probability is affected in the absence of Rbcn3a KO. A similar phenotype was observed in spontaneous activity measured from lateral line organ in zebra-fish Rbcn-3a mutant (Einhorn et al., 2012). In this study, the effect of bafilomycin A1 impacted firing rates more than loss of Rbcn-3a, suggesting that Rbcn-3a loss leads to a partial filling defect.

The defect in SV recycling in Rbcn-3a KO seems to emerge from defective regulation of vesicle acidification. The direct consequence would be NT (re)-filling in the newly formed SVs at the synapse. Apart from extended electrophysiological analysis, it would be interesting to use GFP. based glutamate sensor, a novel probe developed recently to measure the glutamate level (Marvin et al., 2013). By targeting to the SV lumen through an integral protein and adapting the 
sensitivity of the probe to high concentration of luminal glutamate (Hori and Takahashi, 2012), such a probe can be used to measure vesicular content. Additionally, analysis of vesicle shape in electron micrograms can be used as a proxy to get a clue about vesicle fill state. Such an analysis coupled with further electrophysiological recordings from isolated neurons in culture and NT filling measurement will give a concrete idea about the role of Rbcn-3a in regulation of vesicle acidification and thereby NT refilling.

\subsubsection{Rben-3a links endocytosis, acidification and SV maturation}

Rab GTPases Rab3 and Rab27 play a role in SV exocytosis and co-ordinate the release of vesicles at the synapse (reviewed in (Pavlos and Jahn, 2011)). It is not yet known when Rab3GAP and Rab3-GEF are activated/recruited during the SV cycle to actively regulate Rab3. Rbcn3 complex was initially identified as an adaptor that regulates Rab3 cycle, by mediating GTP/GDP conversion through its interaction with Rab3 regulators in SV recycling (Kawabe et al., 2003; Nagano et al., 2002). In the absence of Rbcn-3a, I found that the overall protein level is reduced in the neurons and Rab3 recruitment on SVs is impaired severely. Thus, a putative v-ATPase regulator Rbcn-3a seems to be linking SV maturation step (activating Rab3), vesicle acidification and endocytosis.

Hypothetical model of rbcn-3a functions in SV recycling:

Based on the current knowledge of v-ATPase regulation, identified roles of Rbcn-3a in SV recycling and its molecular interaction with proteins of different machineries in the SV cycle, I hypothesize the following model (Figure 10). Upon stimulation, vesicles are mobilized to the release site to get prepared for the fusion process. It was shown that fully filled vesicle, or, in an independent study, SVs without V1 complex are favored for fusion (Bodzęta et al., 2017; Rost et al., 2015). V1 complex along with Rbcn-3a could be disassociated temporarily from SVs that are ready to fuse at the release sites and Rbcn-3 in the cytosol could mediate hydrolysis of Rab3 bound to GTP through activation/recruitment of Rab3-GAP through yet unknown intermediate interactor. Once the vesicle undergoes fusion, the excessive membrane and protein added are retrieved by one of the several endocytic pathways. My data shows that v-ATPase as well as Rbcn-3a can come to the CCP on the PM or CCV, generated from PM or endosomal 
intermediates. The clathrin coat on the endocytic vesicle blocks the v-ATPase activity (Farsi et al., 2018) and the uncoating of this vesicle releases this inhibition, thereby acting as a checkpoint between retrieval step and acidification/NT refilling. Rbcn-3a promotes the stability of the vATPase complex and its absence results in impaired SV acidification and a delay in recycling process. Once the vesicle undergoes acidification and is filled with NT, it can be ready for the next fusion event. Such vesicles also recruit peripheral protein like Rab3, bound to GTP. Rab3GDP in the cytosol could be activated through Rbcn-3b interaction with Rab3-GEF (Rbcn-3b interacts directly with Rab3-GEF, (Kawabe et al., 2003)) and this can help in GDP-GTP exchange of Rab3. In C. elegans, endophilin was targeted to SVs by a mechanism regulated by Rab3 and Rab3-GEF and predominantly found in the matrix around SV clusters before being targeted to PM by exocytosis (Bai et al., 2010). Endophilin A1 binding to SV membrane enhanced their release efficiency, either transiently after the vesicle is formed by endocytosis or even at the CCV/CCP level and this positive effect was inhibited by interaction with VGLUT1 (Weston et al., 2011). This sequence of events could favor the release of a properly acidified, fully filled SV formed from endocytosis, with the required accessory factors. The model also suggests that individual proteins previously attributed a role in particular step of SV recycling participates in regulating other connected events. This model is speculative, based on available data and links from other studies. Further experiments have to be conducted to test this hypothesis. 


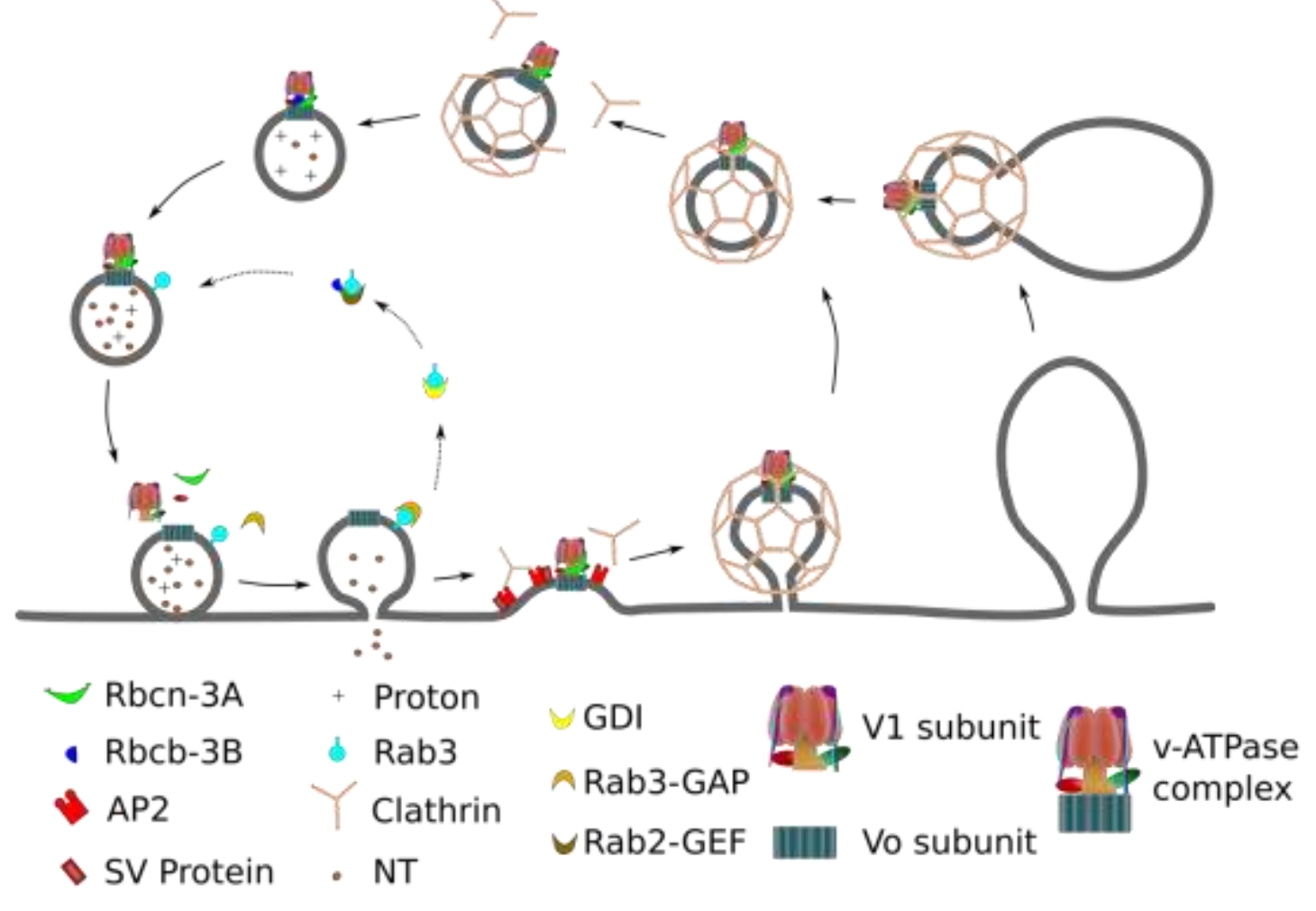

Figure 10 Hypothetical model proposed to explain the role of Rbcn-3a in SV recycling pathway

Rbcn-3a potentially stabilizes the v-ATPase complex on the vesicle during the SV recycling process. Endocytic retrieval from the PM by clathrin dependent or independent mechanism results in the formation of clathrin coated vesicles, which has a fully assembled v-ATPase but is not functional due to the block by clathrin coat. Rbcn-3a may stabilize the v-ATPase assembled already at the coated pits on the plasma membrane. Uncoating reaction releases the block on acidification of vesicles. In the absence of $\mathrm{Rbcn}-3 \mathrm{a}$, SV (re)acidification is impaired and delays the recycling process in the neurons. Once the vesicle is filled with neurotransmitter, Rbcn-3 complex, through the Rbcn-3b subunit can activate the Rab3 recruitment on the vesicle through its interaction with Rab3-GEF. The vesicle with all the required accessory proteins recruited can participate in the next cycle of events. A fully filled vesicle is also favored for release and the disassembly of $V_{1}$ from $V_{0}$ further might be helping this process. The disassembly of $\mathrm{V}$ ATPase on the release ready vesicles along with translocation of Rbcn-3a to the cytosol can mediate the Rab3-GTP hydrolysis on the vesicle before/during fusion through Rab3-GAP. Rbcn-3 complex could thus act as a scaffold to orchestrate the steps in the vesicle recycling pathway. 


\subsubsection{V-ATPase and its regulator at the cross road between cellular trafficking and metabolic pathways}

Analysis of ultrastructure by EM lead to an interesting observation that Rbcn-3a KO neurons accumulated endosome-like-structures, as well as lysosome-like-structures (observed as electron-dense structures in the EM images). It is not clear if the accumulated endosome-likestructures are synaptic endosomes or intermediates formed by the recycling pathway. v-ATPase is not only essential for neurotransmission, but it has a housekeeping role in the general trafficking pathway and Rbcn-3a could have an independent role along with v-ATPase on the trafficking organelle besides SVs at the terminal. Also, function in endosomal maturation process has been suggested by several studies on Rbcn-3a in the other model systems analyzed (Sethi et al., 2010; Tuttle et al., 2014; Yan et al., 2009). Further, other small GTPases (for example, Arf6) have been implicated in re-routing vesicles between the trafficking pathways and silencing of Arf6 leads to an accumulation of endosome-like structure at the expense of reduced vesicles (Tagliatti et al., 2016).

The presence of lysosome-like-structure in the neuronal process by EM was supported by an increase in LAMP1 (lysosome-associated membrane protein 1)-positive structures and an increase in the total protein levels. Since there is a reduction in the integral vesicle protein level and an increase in lysosomal markers, it would be important to check if the recycling defects induced by the absence of Rbcn-3a results in targeting of the proteins for degradation and/or alter the recycling routes in the neuron. Lysosomes are central to macromolecule degradation and nutrient recycling in a cell in general and it is becoming more evident that they are more than a mere garbage accumulation center of the cell. Although much is not yet known about the role of lysosomes in neurons beyond being the degradation pathway target in the cellular trafficking event (reviewed in (Ferguson, 2018)), its role as a signaling station to integrate cues from metabolic and signaling pathways in the cell is clear from several studies (reviewed in (Perera and Zoncu, 2016)). New ideas are emerging about how lysosomes are redistributed between different compartments in a neuron depending on the demand during activity, besides upholding the catabolic function to yield basic building blocks of the cell, especially in times of stress. vATPase on lysosomes has an important role in this metabolic and signaling pathway integration, for example amino acid sensing mTORC1 signaling pathway (Zoncu et al., 2011). Further, 
homolog of Rbcn-3a has a role in glucose dependent assembly/disassembly of v-ATPase on vacuoles (Smardon and Kane, 2007). The additional phenotype observed in Rbcn-3a KO neurons suggests that its v-ATPase regulation activity on lysosomes could be implicated in the metabolic signaling pathway in the cell. Synaptic vesicle cycle has high metabolic demand during activity and this was shown to match the activity-driven stimulation of ATP production (Rangaraju et al., 2014). Activity driven intracellular calcium was proposed to be one of the likely mechanisms that links the two processes; however, the locus of this control and other questions on synaptic metabolism are unanswered. v-ATPase and its regulator might have a role in this coupling between synaptic transmission and metabolism.

\subsubsection{Implication of Rbcn-3a in human physiology}

Homozygous in-frame deletion of 15 nucleotides in DMXL2 (gene encoding for Rbcn-3a) caused a new syndrome, later named as PEPNS. Low levels of DMXL2 in these patients caused complex neurological phenotype, profound abnormalities in glucose metabolism and gonodanotropic axis deficiency due to loss of $\mathrm{GnRH}$ (gonadotropin-releasing hormone) neurons. Reduction in the levels of Rbcn-3a lowered both the constitutive as well as glucose induced secretion of insulin (Tata et al., 2014). In a follow-up study, the defects in maturation of $\mathrm{GnRH}$ neuron was attributed to diminished response by the receptor to the hormone kisspeptin, which controls $\mathrm{GnRH}$ secretion at puberty (Tata et al., 2017). One of patients reported by Tata and colleagues also developed hearing loss during early adulthood. Interestingly, sequencing a large Chinese family with several members affected by an autosomal-dominant nonsyndromic hearing loss revealed DMXL2 as deafness-associated gene in humans (Chen et al., 2017). This is in line with the deafness phenotype reported in VGLUT3 KO mice, caused by the absence of glutamate release (Seal et al., 2008). Taking the results from previous work on homologs of Rbcn-3a, together with hints from my thesis work, Rbcn-3a affects reactivation of the $\mathrm{GnRH}$ neuronal network as well as the age-related deafness, mostly by controlling processes like SV recycling and receptor trafficking, while the metabolic phenotype in the patients could be attributed to effects mediated through lysosome and signaling pathway regulation. 


\section{Summary and Outlook}

Synaptic vesicle cycle is one of the finely controlled cell biological processes. Molecular steps in this pathway have been studied intensively, especially the steps of exo- and endocytosis and the overall mechanism of how it proceeds is largely understood. The goal of my thesis was to further the understanding of some of the missing links in the SV recycling mechanism. The intermediate steps like coupling of exocytosis to endocytosis, how and when re-acidification of a newly endocytosed vesicle occurs and how a fusion competent SV is made after local endocytic reformation of the vesicle at the synapse are some of the open questions that remain unanswered. Moreover, different protein machinery has been studied in the context of specific steps in the SV cycle, but how the proteins are made available at the right time in defined location is not fully understood.

A wide range of model systems has been used to study SV recycling and many innovative tools have been developed over years to dissect the complete recycling mechanism at the synapse. Although some of these questions are difficult to resolve within the scope of the available tools and resources, the aims of this thesis were crafted with these questions in mind as the bigger picture. The main outcomes of the thesis-related research include finding a new role for an endocytic protein in exocytosis as well as how a regulator of v-ATPase links endocytosis to vesicle refilling/acidification and forming fusion competent vesicles. The conclusions derived from the obtained results are summarized in the next paragraphs.

The first study reports a new role for endophilin in exocytosis, independent of its role in endocytic retrieval mechanisms. The lack of endophilin reduces the LDCV exocytosis in adrenal chromaffin cells, a neurosecretory model system commonly employed to study regulated exocytosis. Specifically, absence of endophilin affects the vesicle recruitment, priming and fusion of vesicles during exocytosis. This was independent of membrane and protein recycling by endocytic mechanisms. Additionally, loss of endophilin did not affect the LDCV number per cell or area in chromaffin cells, in contrast to neurons, where the number of SVs is reduced due to defective recycling and accumulation of coated intermediates. Further, none of the major proteins involved in fusion reaction or the retrieval process were affected by the absence of endophilin in chromaffin cells. The regulatory role of endophilin in exocytosis was mediated through its $\mathrm{SH} 3$ domain, potentially by protein-protein interaction of the $\mathrm{SH} 3$ domain. The role in vesicle recruitment and 
release, at least in part was mediated through endophilin's interaction with an adaptor protein intersectin, which is involved in LDCV exocytosis and implicated in exo-endocytosis coupling mechanisms. This study showed that endophilin has a direct role in vesicle exocytosis in chromaffin cells as well as uncovered how protein-protein interaction helps to compartmentalize endocytic proteins and make them available at the right time for the events in vesicle trafficking pathways. One possible mechanism of action of the endophilin-intersectin interaction could be through regulation of actin cytoskeleton, which plays important role in vesicle mobilization and making release sites available. The results may be extrapolated to neuronal synapses, although the mechanism of action in neurons might differ to a certain extent due to increased complexity imposed by fast local recycling mechanisms.

In the second study, I focused on understanding how the process of v-ATPase regulation is linked to endocytosis and vesicle maturation steps in SV recycling process. We considered Rbcn-3a to be a putative v-ATPase regulator, and I characterized its role in SV recycling in neurons. Rbcn$3 a$ is a vesicle-associated protein, found on both SV and CCV at the synapse, as well as organelles in trafficking pathways, e.g. endosomes and lysosomes, which rely on organelle acidification for their function. Loss of Rbcn-3a caused a reduction in number of SVs without affecting the overall synapse morphology, reduced the total integral vesicle protein levels in the neurons and finally impaired its activity. Presynaptic activity monitored using $\mathrm{pH}$ sensitive pHluorin tagged to VGLUT1 luminal domain showed that the recovery after stimulation was delayed in the absence of Rbcn-3a KO neurons. This was not due to a defect in endocytic retrieval mechanism; since FM dye uptake was unchanged as well the levels of endocytic factors were not altered in the KO neurons. Thus, the delayed recovery could be due to defective re-acidification of vesicles and accumulation in an 'alkaline trapped' state. As a consequence, recruitment of Rab3 on SVs was reduced and its stability on SV was impaired in Rbcn-3a KO neurons. Interestingly, blocking vesicle acidification with v-ATPase blocker (bafilomycin A1) also resulted in reduced Rab3 recruitment on vesicles. This suggests that improper vesicle acidification and NT refilling will affect the recruitment of peripheral factors, thereby affecting the formation of fusion competent vesicles. Additionally, synapses also accumulated endosome-like-structures and lysosomes, suggesting that loss of Rbcn-3a affected constitutive cellular trafficking pathways as well. The results from the second study suggest that Rbcn-3a affects several trafficking organelles in the neurons through regulation of $v$-ATPase function. 
The first study adds to the mechanism of coupling between exocytosis and endocytosis steps and a new role for endophilin, classically assumed an endocytic protein. The second study on the role of Rbcn-3a adds an interesting link between various cellular processes including acidification, endocytosis, SV maturation and endo-lysosomal trafficking pathway. Both the studies reinstate how a particular protein may impact more than one mechanism depending on its distribution in the cell, which organelle is the site of its action and the local interaction partners, apart from cellular environmental factors.

\subsection{Future Perspective}

In cellular trafficking processes, most reactions occurring in a cell are spatially and temporally controlled, which raises the question about how the proteins and factors required are made available for this reaction sufficiently at the right time and in correct location (Rizzoli, 2014). The idea that one protein has one function or alternatively, a process is carried out only by a set of proteins is then limiting. Rather, recent studies have been revealing a new-found role for proteins with set primary function. For example, endophilin was considered to be an endocytic adaptor for over 30 years now, but more recent studies are revealing unexpected role in autophagy (Murdoch et al., 2016), association with SV cluster (Bai et al., 2010), vesicle release modulation (Weston et al., 2011) and the findings from my thesis add a direct role for endophilin in exocytosis. This argues in favor of an idea that the large pool of vesicles at the terminal help to buffer the proteins, so that they are available for cellular reactions abundantly at the right time. Instead of having one main function, it is likely that the proteins participate in multiple steps of vesicle recycling at the synapse. A lot of well-studied synaptic proteins are being revisited to understand their secondary roles in the cellular mechanisms, since the already defined properties are not entirely sufficient to explain the phenotype manifested when their levels are altered in the model systems or human patients.

Although we have reported a new direct role for endophilin in exocytosis, the mechanism of action remains to be worked out in detail in the future. It would be interesting to test if the effect of endophilin is mediated only through its interaction with intersectin. Additionally, it can also involve the interaction with dynamin, which is already known to have a role in fusion pore dynamics 
regulation. Dynamin is recruited to the membrane through BAR domain containing proteins; therefore, detailed fusion pore dynamics studies will shed light on the involvement of endophilin in this process (similar to (Shin et al., 2018)). Further, endocytic mechanisms are not well understood in chromaffin cells. Using mouse models of endocytosis like endophilin TKO and dynamin KOs, among others, in combination with capacitance measurement using perforated patch will help to work out these pathways in the chromaffin cells (as discussed in (Smith and Neher, 1997)). It is also known that calcium regulates endocytosis and the availability of proteins involved by activity dependent phosphorylation. Additionally, endophilin interacts directly with voltage-gated $\mathrm{Ca}^{2+}$ channels (Chen et al., 2003). So it would be good to check the influence of calcium levels on the function of endophilin in exocytosis, for example by measuring the calcium dependence of secretion in the absence of endophilins (Pinheiro et al., 2014). Lastly, membrane lipids can affect the function of endophilin as well, since it would affect the BAR domain mediated roles of the protein in exo- and endocytosis. Further research will be required to answer some of these aspects as well as to verify if the role of endophilin in exocytosis is limited to chromaffin cells or can be extended to neurons in the central nervous system.

The second study reaffirmed the evolutionarily established role for Rbcn-3a in v-ATPase regulation. Interestingly, while the protein is involved in coupling $V_{0}$ and $V_{1}$ on vacuoles in yeast in response to nutrient availability, it has a more complex function in the SV recycling process, where it has an additional role to link multiple steps in the recycling pathway. The initial characterization done as a part of this thesis sheds some light on role of Rbcn-3a in recycling mechanisms; however, a lot work remains to be done. Although my results support a role for regulation of $v$-ATPase on SVs, the precise mechanism of how this is done needs to be experimentally verified in the neurons. The idea that $v$-ATPase regulation occurs by disassembly/assembly cycle in the synapse is being debated and Rbcn-3a could regulate $v$ ATPase through alternative unknown mechanisms. Studying the v-ATPase assembly status on the vesicles and their activity will help to understand the regulatory mechanism of action of Rbcn3a. Along this line, fluorescence recovery after photo bleaching was used to explain certain aspects of v-ATPase functioning at the synapse (Bodzęta et al., 2017) and the technique could be employed to understand the dynamics of Rbcn-3a together with v-ATPase in the recycling pathway. Measuring the vesicular NT content will further support the idea that the protein regulates vesicle acidification and thereby co-ordinates NT refilling step with maturation process. 
Strikingly, the link between acidification and maturation through Rbcn-3a provides an elegant mechanism to link Rab3 cycle to SV recycling process; however, the hypothesis needs to be investigated further. It is interesting to note that patients with alterations in the gene encoding for Rbcn-3a and Rab-GAP have overlapping phenotypes (unpublished data from De Roux lab, Paris), which reinstates the link between the role of Rbcn-3a in acidification regulation as well as trafficking. Almost nothing is known about structure and domain organization of Rbcn-3a and it would be an important piece of the puzzle in the attempt to get a complete picture of the protein's role in cellular processes. Apart from SV recycling, it would be crucial to study the role of Rbcn$3 a$ in LDCV recycling pathway in the neurons, since the patients with PEPNS have neuroendocrine manifestations and impaired glucose metabolism.

The focus of my study for this thesis was limited to SV recycling; however, role of Rbcn-3a in the constitutive membrane trafficking pathway through the endo-lysosomal system needs to be explored in the future. v-ATPase is central to the functioning of this pathway, so the regulatory role of Rbcn-3a in specific cell-types and receptor trafficking should be investigated, especially since the human patients with altered DMXL2 gene dosage have reduced response to ligand (kisspeptin) that controls GnRH neuron secretion (Tata et al., 2017).

Another curious observation from my study on Rbcn-3a involves changes in the lysosomes, which are the center where endocytic, secretory and autophagy pathways converge. Also, impaired glucose metabolism could be a consequence of changes in the metabolic signaling pathways and nutrient sensing pathways centered on the lysosomes. Some preliminary analyses of lysosomes in the absence of Rbcn-3a point towards their accumulation and an impairment of their acidification. Further analysis of signaling pathways will help us to gain insight into the role of Rbcn-3a in lysosome centered signaling pathways. The outcome of the signaling pathway analysis will be crucial to explain some of the observed phenotypes in PEPNS patients, and potentially suggest treatments, at least for some of their symptoms.

Taking all discussed points together, this study opened many interesting questions, which are fundamental and relevant to physiology. The study also brings up a point as to how an evolutionarily conserved protein evolves its role with complexity of the organism, organelle at quest and interaction partners involved in the cellular processes. Thus, understanding the 
mechanism of v-ATPase regulation, along with the role of Rbcn-3 complex will hopefully link some fundamental concepts of cell biology to neuroscience and physiology.

Scientific research endeavors have broadened our knowledge and understanding of the brain, yet we have not fully understood some of its fundamental concepts. This thesis was carried out with a desire to learn some of these fundamental concepts in neuronal cell biology, which are important stepping stones to understand the brain function in health and disease.

\section{References}

Alabi, A.A., and Tsien, R.W. (2012). Synaptic Vesicle Pools and Dynamics. Cold Spring Harb. Perspect. Biol. 4, a013680.

Anantharam, A., Bittner, M.A., Aikman, R.L., Stuenkel, E.L., Schmid, S.L., Axelrod, D., and Holz, R.W. (2011). A new role for the dynamin GTPase in the regulation of fusion pore expansion. Mol. Biol. Cell 22, 1907-1918.

Anderson, R.G., and Orci, L. (1988). A view of acidic intracellular compartments. J. Cell Biol. 106, 539-543.

Anderson, R.G., Falck, J.R., Goldstein, J.L., and Brown, M.S. (1984). Visualization of acidic organelles in intact cells by electron microscopy. Proc. Natl. Acad. Sci. U. S. A. 81, 4838-4842.

Arata, Y., Baleja, J.D., and Forgac, M. (2002). Localization of Subunits D, E, and G in the Yeast V-ATPase Complex Using Cysteine-Mediated Cross-Linking to Subunit B. Biochemistry 41, 11301-11307.

Armbrüster, A., Hohn, C., Hermesdorf, A., Schumacher, K., Börsch, M., and Grüber, G. (2005). Evidence for major structural changes in subunit $C$ of the vacuolar ATPase due to nucleotide binding. FEBS Lett. 579, 1961-1967.

Artalejo, C.R., Henley, J.R., McNiven, M.A., and Palfrey, H.C. (1995). Rapid endocytosis coupled to exocytosis in adrenal chromaffin cells involves $\mathrm{Ca2}+$, GTP, and dynamin but not clathrin. Proc. Natl. Acad. Sci. 92, 8328-8332.

Artalejo, C.R., Elhamdani, A., and Palfrey, H.C. (2002). Sustained stimulation shifts the mechanism of endocytosis from dynamin-1-dependent rapid endocytosis to clathrin- and dynamin-2-mediated slow endocytosis in chromaffin cells. Proc. Natl. Acad. Sci. U. S. A. 99, 6358-6363.

Ashery, U., Varoqueaux, F., Voets, T., Betz, A., Thakur, P., Koch, H., Neher, E., Brose, N., and Rettig, J. (2000). Munc13-1 acts as a priming factor for large dense-core vesicles in bovine chromaffin cells. EMBO J. 19, 3586-3596. 
Atluri, P.P., and Ryan, T.A. (2006). The Kinetics of Synaptic Vesicle Reacidification at Hippocampal Nerve Terminals. J. Neurosci. 26, 2313-2320.

Augustin, I., Rosenmund, C., Südhof, T.C., and Brose, N. (1999). Munc13-1 is essential for fusion competence of glutamatergic synaptic vesicles. Nature 400, 457-461.

Azarnia Tehran, D., Kuijpers, M., and Haucke, V. (2018). Presynaptic endocytic factors in autophagy and neurodegeneration. Curr. Opin. Neurobiol. 48, 153-159.

Bai, J., Hu, Z., Dittman, J.S., Pym, E.C.G., and Kaplan, J.M. (2010). Endophilin Functions as a Membrane-Bending Molecule and Is Delivered to Endocytic Zones by Exocytosis. Cell 143, 430441.

Betz, A., Thakur, P., Junge, H.J., Ashery, U., Rhee, J.S., Scheuss, V., Rosenmund, C., Rettig, J., and Brose, N. (2001). Functional interaction of the active zone proteins Munc13-1 and RIM1 in synaptic vesicle priming. Neuron 30,183-196.

Beyenbach, K.W., and Wieczorek, H. (2006). The V-type H+ ATPase: molecular structure and function, physiological roles and regulation. J. Exp. Biol. 209, 577-589.

Blakely, R.D., and Edwards, R.H. (2012). Vesicular and plasma membrane transporters for neurotransmitters. Cold Spring Harb. Perspect. Biol. 4.

Blasi, J., Chapman, E.R., Link, E., Binz, T., Yamasaki, S., De Camilli, P., Südhof, T.C., Niemann, H., and Jahn, R. (1993a). Botulinum neurotoxin A selectively cleaves the synaptic protein SNAP25. Nature 365, 160-163.

Blasi, J., Chapman, E.R., Yamasaki, S., Binz, T., Niemann, H., and Jahn, R. (1993b). Botulinum neurotoxin $\mathrm{C} 1$ blocks neurotransmitter release by means of cleaving HPC-1/syntaxin. EMBO J. 12, 4821-4828.

Bloom, O., Evergren, E., Tomilin, N., Kjaerulff, O., Löw, P., Brodin, L., Pieribone, V.A., Greengard, P., and Shupliakov, O. (2003). Colocalization of synapsin and actin during synaptic vesicle recycling. J. Cell Biol. 161, 737-747.

Bodzęta, A., Kahms, M., and Klingauf, J. (2017). The Presynaptic v-ATPase Reversibly Disassembles and Thereby Modulates Exocytosis but Is Not Part of the Fusion Machinery. Cell Rep. 20, 1348-1359.

Boucrot, E., Ferreira, A.P.A., Almeida-Souza, L., Debard, S., Vallis, Y., Howard, G., Bertot, L., Sauvonnet, N., and McMahon, H.T. (2015). Endophilin marks and controls a clathrin-independent endocytic pathway. Nature 517, 460-465.

Brodal, P. (2010). The Central Nervous System (New York: Oxford University Press).

Brose, N., Hofmann, K., Hata, Y., and Südhof, T.C. (1995). Mammalian homologues of Caenorhabditis elegans unc-13 gene define novel family of C2-domain proteins. J. Biol. Chem. 270, 25273-25280. 
Cao, M., Milosevic, I., Giovedi, S., and De Camilli, P. (2014). Upregulation of Parkin in endophilin mutant mice. J. Neurosci. Off. J. Soc. Neurosci. 34, 16544-16549.

Chapman, E.R. (2008). How does synaptotagmin trigger neurotransmitter release? Annu. Rev. Biochem. 77, 615-641.

Chen, D.-Y., Liu, X.-F., Lin, X.-J., Zhang, D., Chai, Y.-C., Yu, D.-H., Sun, C.-L., Wang, X.-L., Zhu, W.-D., Chen, Y., et al. (2017). A dominant variant in DMXL2 is linked to nonsyndromic hearing loss. Genet. Med. Off. J. Am. Coll. Med. Genet. 19, 553-558.

Chen, Y., Deng, L., Maeno-Hikichi, Y., Lai, M., Chang, S., Chen, G., and Zhang, J. (2003). Formation of an Endophilin-Ca2+ Channel Complex Is Critical for Clathrin-Mediated Synaptic Vesicle Endocytosis. Cell 115, 37-48.

Clary, D.O., Griff, I.C., and Rothman, J.E. (1990). SNAPs, a family of NSF attachment proteins involved in intracellular membrane fusion in animals and yeast. Cell 61, 709-721.

Clayton, E.L., Evans, G.J.O., and Cousin, M.A. (2008). Bulk Synaptic Vesicle Endocytosis Is Rapidly Triggered during Strong Stimulation. J. Neurosci. Off. J. Soc. Neurosci. 28, 6627-6632.

Coppola, T., Magnin-Luthi, S., Perret-Menoud, V., Gattesco, S., Schiavo, G., and Regazzi, R. (2001). Direct interaction of the Rab3 effector RIM with Ca2+ channels, SNAP-25, and synaptotagmin. J. Biol. Chem. 276, 32756-32762.

Cousin, M.A., and Robinson, P.J. (2001). The dephosphins: dephosphorylation by calcineurin triggers synaptic vesicle endocytosis. Trends Neurosci. 24, 659-665.

Cremona, O., Di Paolo, G., Wenk, M.R., Lüthi, A., Kim, W.T., Takei, K., Daniell, L., Nemoto, Y., Shears, S.B., Flavell, R.A., et al. (1999). Essential Role of Phosphoinositide Metabolism in Synaptic Vesicle Recycling. Cell 99, 179-188.

Daumke, O., Roux, A., and Haucke, V. (2014). BAR domain scaffolds in dynamin-mediated membrane fission. Cell 156, 882-892.

De Camilli, P., and Jahn, R. (1990). Pathways to regulated exocytosis in neurons. Annu. Rev. Physiol. 52, 625-645.

Di Paolo, G., and De Camilli, P. (2006). Phosphoinositides in cell regulation and membrane dynamics. Nature 443, 651-657.

Diril, M.K., Wienisch, M., Jung, N., Klingauf, J., and Haucke, V. (2006). Stonin 2 is an AP-2dependent endocytic sorting adaptor for synaptotagmin internalization and recycling. Dev. Cell 10, 233-244.

Dulubova, I., Sugita, S., Hill, S., Hosaka, M., Fernandez, I., Südhof, T.C., and Rizo, J. (1999). A conformational switch in syntaxin during exocytosis: role of munc18. EMBO J. 18, 4372-4382. 
Dulubova, I., Lou, X., Lu, J., Huryeva, I., Alam, A., Schneggenburger, R., Südhof, T.C., and Rizo, J. (2005). A Munc13/RIM/Rab3 tripartite complex: from priming to plasticity? EMBO J. 24, 28392850.

Edeling, M.A., Smith, C., and Owen, D. (2006). Life of a clathrin coat: insights from clathrin and AP structures. Nat. Rev. Mol. Cell Biol. 7, 32-44.

Edwards, R.H. (2007). The neurotransmitter cycle and quantal size. Neuron 55, 835-858.

Egashira, Y., Takase, M., and Takamori, S. (2015). Monitoring of Vacuolar-Type H+ ATPaseMediated Proton Influx into Synaptic Vesicles. J. Neurosci. 35, 3701-3710.

Einhorn, Z., Trapani, J.G., Liu, Q., and Nicolson, T. (2012). Rabconnectin3a promotes stable activity of the $\mathrm{H}+$-pump on synaptic vesicles in hair cells. J. Neurosci. Off. J. Soc. Neurosci. 32, 11144-11156.

Evergren, E., Benfenati, F., and Shupliakov, O. (2007). The synapsin cycle: A view from the synaptic endocytic zone. J. Neurosci. Res. 85, 2648-2656.

Farsad, K., Ringstad, N., Takei, K., Floyd, S.R., Rose, K., and Camilli, P.D. (2001). Generation of high curvature membranes mediated by direct endophilin bilayer interactions. J Cell Biol 155, 193-200.

Farsi, Z., Preobraschenski, J., Bogaart, G. van den, Riedel, D., Jahn, R., and Woehler, A. (2016). Single-vesicle imaging reveals different transport mechanisms between glutamatergic and GABAergic vesicles. Science 351, 981-984.

Farsi, Z., Jahn, R., and Woehler, A. (2017). Proton electrochemical gradient: Driving and regulating neurotransmitter uptake. BioEssays 39, 1600240.

Farsi, Z., Gowrisankaran, S., Krunic, M., Rammner, B., Woehler, A., Lafer, E.M., Mim, C., Jahn, R., and Milosevic, I. (2018). Clathrin coat controls synaptic vesicle acidification by blocking vacuolar ATPase activity.

Ferguson, S.M. (2018). Neuronal lysosomes. Neurosci. Lett.

Ferguson, S.M., and Camilli, P.D. (2012). Dynamin, a membrane-remodelling GTPase. Nat. Rev. Mol. Cell Biol. 13, 75-88.

Ferguson, S., Raimondi, A., Paradise, S., Shen, H., Mesaki, K., Ferguson, A., Destaing, O., Ko, G., Takasaki, J., Cremona, O., et al. (2009). Coordinated Actions of Actin and BAR Proteins Upstream of Dynamin at Endocytic Clathrin-Coated Pits. Dev. Cell 17, 811-822.

Ferguson, S.M., Brasnjo, G., Hayashi, M., Wolfel, M., Collesi, C., Giovedi, S., Raimondi, A., Gong, L.-W., Ariel, P., Paradise, S., et al. (2007). A Selective Activity-Dependent Requirement for Dynamin 1 in Synaptic Vesicle Endocytosis. Science 316, 570-574. 
Fischer von Mollard, G., Mignery, G.A., Baumert, M., Perin, M.S., Hanson, T.J., Burger, P.M., Jahn, R., and Sudhof, T.C. (1990). rab3 is a small GTP-binding protein exclusively localized to synaptic vesicles. Proc. Natl. Acad. Sci. 87, 1988-1992.

Ford, M.G., Pearse, B.M., Higgins, M.K., Vallis, Y., Owen, D.J., Gibson, A., Hopkins, C.R., Evans, P.R., and McMahon, H.T. (2001). Simultaneous binding of Ptdlns(4,5)P2 and clathrin by AP180 in the nucleation of clathrin lattices on membranes. Science 291, 1051-1055.

Ford, M.G.J., Mills, I.G., Peter, B.J., Vallis, Y., Praefcke, G.J.K., Evans, P.R., and McMahon, H.T. (2002). Curvature of clathrin-coated pits driven by epsin. Nature 419, 361-366.

Forgac, M. (2007). Vacuolar ATPases: rotary proton pumps in physiology and pathophysiology. Nat. Rev. Mol. Cell Biol. 8, 917-929.

Forgac, M., Cantley, L., Wiedenmann, B., Altstiel, L., and Branton, D. (1983). Clathrin-coated vesicles contain an ATP-dependent proton pump. Proc. Natl. Acad. Sci. U. S. A. 80, 1300-1303.

Frost, A., Unger, V.M., and De Camilli, P. (2009). The BAR Domain Superfamily: MembraneMolding Macromolecules. Cell 137, 191-196.

Fulop, T., Doreian, B., and Smith, C. (2008). Dynamin I plays dual roles in the activity-dependent shift in exocytic mode in mouse adrenal chromaffin cells. Arch. Biochem. Biophys. 477, 146-154.

Galas, M.C., Chasserot-Golaz, S., Dirrig-Grosch, S., and Bader, M.F. (2000). Presence of dynamin-syntaxin complexes associated with secretory granules in adrenal chromaffin cells. J. Neurochem. 75, 1511-1519.

Gallop, J.L., Jao, C.C., Kent, H.M., Butler, P.J.G., Evans, P.R., Langen, R., and McMahon, H.T. (2006). Mechanism of endophilin N-BAR domain-mediated membrane curvature. EMBO J. 25, 2898-2910.

Gandhi, S.P., and Stevens, C.F. (2003). Three modes of synaptic vesicular recycling revealed by single-vesicle imaging. Nature 423, 607-613.

Geppert, M., Archer, B.T., and Südhof, T.C. (1991). Synaptotagmin II. A novel differentially distributed form of synaptotagmin. J. Biol. Chem. 266, 13548-13552.

Geppert, M., Goda, Y., Hammer, R.E., Li, C., Rosahl, T.W., Stevens, C.F., and Südhof, T.C. (1994). Synaptotagmin I: a major Ca2+ sensor for transmitter release at a central synapse. Cell 79, 717-727.

von Gersdorff, H., and Matthews, G. (1994). Inhibition of endocytosis by elevated internal calcium in a synaptic terminal. Nature 370, 652-655.

Giachino, C., Lantelme, E., Lanzetti, L., Saccone, S., Bella Valle, G., and Migone, N. (1997). A novel SH3-containing human gene family preferentially expressed in the central nervous system. Genomics 41, 427-434. 
Gil, A., Rueda, J., Viniegra, S., and Gutiérrez, L.M. (2000). The F-actin cytoskeleton modulates slow secretory components rather than readily releasable vesicle pools in bovine chromaffin cells. Neuroscience 98, 605-614.

Giovanni, J.D., Boudkkazi, S., Mochida, S., Bialowas, A., Samari, N., Lévêque, C., Youssouf, F., Brechet, A., Iborra, C., Maulet, Y., et al. (2010). V-ATPase Membrane Sector Associates with Synaptobrevin to Modulate Neurotransmitter Release. Neuron 67, 268-279.

Giovedì, S., Darchen, F., Valtorta, F., Greengard, P., and Benfenati, F. (2004). Synapsin Is a Novel Rab3 Effector Protein on Small Synaptic Vesicles. J. Biol. Chem. 279, 43769-43779.

Glick, B.S., and Rothman, J.E. (1987). Possible role for fatty acyl-coenzyme A in intracellular protein transport. Nature 326, 309-312.

Graham, M.E., O'Callaghan, D.W., McMahon, H.T., and Burgoyne, R.D. (2002). Dynamindependent and dynamin-independent processes contribute to the regulation of single vesicle release kinetics and quantal size. Proc. Natl. Acad. Sci. 99, 7124-7129.

Greengard, P., Valtorta, F., Czernik, A., and Benfenati, F. (1993). Synaptic vesicle phosphoproteins and regulation of synaptic function. Science 259, 780-785.

Grønborg, M., Pavlos, N.J., Brunk, I., Chua, J.J.E., Münster-Wandowski, A., Riedel, D., AhnertHilger, G., Urlaub, H., and Jahn, R. (2010). Quantitative comparison of glutamatergic and GABAergic synaptic vesicles unveils selectivity for few proteins including MAL2, a novel synaptic vesicle protein. J. Neurosci. Off. J. Soc. Neurosci. 30, 2-12.

Guan, R., Han, D., Harrison, S.C., and Kirchhausen, T. (2010). Structure of the PTEN-like region of auxilin, a detector of clathrin-coated vesicle budding. Struct. Lond. Engl. 1993 18, 1191-1198.

Hallermann, S., Fejtova, A., Schmidt, H., Weyhersmüller, A., Silver, R.A., Gundelfinger, E.D., and Eilers, J. (2010). Bassoon speeds vesicle reloading at a central excitatory synapse. Neuron 68, 710-723.

Hata, Y., Slaughter, C.A., and Südhof, T.C. (1993). Synaptic vesicle fusion complex contains unc18 homologue bound to syntaxin. Nature 366, 347-351.

Haucke, V., Neher, E., and Sigrist, S.J. (2011). Protein scaffolds in the coupling of synaptic exocytosis and endocytosis. Nat. Rev. Neurosci. 12, 127-138.

Hayashi, M., Raimondi, A., O'Toole, E., Paradise, S., Collesi, C., Cremona, O., Ferguson, S.M., and De Camilli, P. (2008). Cell- and stimulus-dependent heterogeneity of synaptic vesicle endocytic recycling mechanisms revealed by studies of dynamin 1-null neurons. Proc. Natl. Acad. Sci. U. S. A. 105, 2175-2180.

Helenius, A., Mellman, I., Wall, D., and Hubbard, A. (1983). Endosomes. Trends Biochem. Sci. 8, 245-250. 
Henne, W.M., Boucrot, E., Meinecke, M., Evergren, E., Vallis, Y., Mittal, R., and McMahon, H.T. (2010). FCHo proteins are nucleators of clathrin-mediated endocytosis. Science 328, 1281-1284.

Heuser, J.E. (1973). EVIDENCE FOR RECYCLING OF SYNAPTIC VESICLE MEMBRANE DURING TRANSMITTER RELEASE AT THE FROG NEUROMUSCULAR JUNCTION. J. Cell Biol. 57, 315-344.

Heuser, J.E., and Reese, T.S. (1973). Evidence for recycling of synaptic vesicle membrane during transmitter release at the frog neuromuscular junction. J. Cell Biol. 57, 315-344.

de Heuvel, E., Bell, A.W., Ramjaun, A.R., Wong, K., Sossin, W.S., and McPherson, P.S. (1997). Identification of the Major Synaptojanin-binding Proteins in Brain. J. Biol. Chem. 272, 8710-8716.

Hiesinger, P.R., Fayyazuddin, A., Mehta, S.Q., Rosenmund, T., Schulze, K.L., Zhai, R.G., Verstreken, P., Cao, Y., Zhou, Y., Kunz, J., et al. (2005). The v-ATPase V0 Subunit a1 Is Required for a Late Step in Synaptic Vesicle Exocytosis in Drosophila. Cell 121, 607-620.

Hnasko, T.S., and Edwards, R.H. (2012). Neurotransmitter Corelease: Mechanism and Physiological Role. Annu. Rev. Physiol. 74, 225-243.

Holt, M., Cooke, A., Wu, M.M., and Lagnado, L. (2003). Bulk membrane retrieval in the synaptic terminal of retinal bipolar cells. J. Neurosci. Off. J. Soc. Neurosci. 23, 1329-1339.

de Hoop, M.J., Huber, L.A., Stenmark, H., Williamson, E., Zerial, M., Parton, R.G., and Dotti, C.G. (1994). The involvement of the small GTP-binding protein Rab5a in neuronal endocytosis. Neuron 13, 11-22.

Hori, T., and Takahashi, T. (2012). Kinetics of Synaptic Vesicle Refilling with Neurotransmitter Glutamate. Neuron 76, 511-517.

Hosoi, N., Holt, M., and Sakaba, T. (2009). Calcium Dependence of Exo- and Endocytotic Coupling at a Glutamatergic Synapse. Neuron 63, 216-229.

Huotari, J., and Helenius, A. (2011). Endosome maturation. EMBO J. 30, 3481-3500.

Hurtado-Lorenzo, A., Skinner, M., Annan, J.E., Futai, M., Sun-Wada, G.-H., Bourgoin, S., Casanova, J., Wildeman, A., Bechoua, S., Ausiello, D.A., et al. (2006). V-ATPase interacts with ARNO and Arf6 in early endosomes and regulates the protein degradative pathway. Nat. Cell Biol. 8, 124-136.

Hussain, N.K., Jenna, S., Glogauer, M., Quinn, C.C., Wasiak, S., Guipponi, M., Antonarakis, S.E., Kay, B.K., Stossel, T.P., Lamarche-Vane, N., et al. (2001). Endocytic protein intersectin-I regulates actin assembly via Cdc42 and N-WASP. Nat. Cell Biol. 3, 927-932.

Inoue, T., and Forgac, M. (2005). Cysteine-mediated Cross-linking Indicates That Subunit C of the V-ATPase Is in Close Proximity to Subunits E and G of the V1 Domain and Subunit a of the V0 Domain. J. Biol. Chem. 280, 27896-27903. 
Itoh, T., and De Camilli, P. (2006). BAR, F-BAR (EFC) and ENTH/ANTH domains in the regulation of membrane-cytosol interfaces and membrane curvature. Biochim. Biophys. Acta 1761, 897912.

Itoh, T., Erdmann, K.S., Roux, A., Habermann, B., Werner, H., and De Camilli, P. (2005). Dynamin and the actin cytoskeleton cooperatively regulate plasma membrane invagination by BAR and FBAR proteins. Dev. Cell 9, 791-804.

Iwata, M., Imamura, H., Stambouli, E., Ikeda, C., Tamakoshi, M., Nagata, K., Makyio, H., Hankamer, B., Barber, J., Yoshida, M., et al. (2004). Crystal structure of a central stalk subunit C and reversible association/dissociation of vacuole-type ATPase. Proc. Natl. Acad. Sci. U. S. A. 101, 59-64.

Jahn, R., and Fasshauer, D. (2012). Molecular machines governing exocytosis of synaptic vesicles. Nature 490, 201-207.

Jahn, R., Lang, T., and Südhof, T.C. (2003). Membrane Fusion. Cell 112, 519-533.

Jähne, S., Rizzoli, S.O., and Helm, M.S. (2015). The structure and function of presynaptic endosomes. Exp. Cell Res. 335, 172-179.

James, D.J., and Martin, T.F.J. (2013). CAPS and Munc13: CATCHRs that SNARE Vesicles. Front. Endocrinol. 4, 187.

Jefferies, K.C., Cipriano, D.J., and Forgac, M. (2008). Function, structure and regulation of the vacuolar $(\mathrm{H}+)$-ATPases. Arch. Biochem. Biophys. 476, 33-42.

Johnson, R.G. (1988). Accumulation of biological amines into chromaffin granules: a model for hormone and neurotransmitter transport. Physiol. Rev. 68, 232-307.

Kaeser, P.S., Deng, L., Wang, Y., Dulubova, I., Liu, X., Rizo, J., and Südhof, T.C. (2011). RIM proteins tether $\mathrm{Ca} 2+$ channels to presynaptic active zones via a direct PDZ-domain interaction. Cell 144, 282-295.

Kane, P.M. (1995). Disassembly and reassembly of the yeast vacuolar $\mathrm{H}(+)$-ATPase in vivo. J. Biol. Chem. 270, 17025-17032.

Kawabe, H., Sakisaka, T., Yasumi, M., Shingai, T., Izumi, G., Nagano, F., Deguchi-Tawarada, M., Takeuchi, M., Nakanishi, H., and Takai, Y. (2003). A novel rabconnectin-3-binding protein that directly binds a GDP/GTP exchange protein for Rab3A small G protein implicated in Ca2+dependent exocytosis of neurotransmitter. Genes Cells 8, 537-546.

Kawasaki, F., Hazen, M., and Ordway, R.W. (2000). Fast synaptic fatigue in shibire mutants reveals a rapid requirement for dynamin in synaptic vesicle membrane trafficking. Nat. Neurosci. 3, 859-860. 
Kettner, C., Bertl, A., Obermeyer, G., Slayman, C., and Bihler, H. (2003). Electrophysiological Analysis of the Yeast V-Type Proton Pump: Variable Coupling Ratio and Proton Shunt. Biophys. J. 85, 3730-3738.

Khodthong, C., Kabachinski, G., James, D.J., and Martin, T.F.J. (2011). Munc13 homology domain-1 in CAPS/UNC31 mediates SNARE binding required for priming vesicle exocytosis. Cell Metab. 14, 254-263.

Kirchhausen, T. (2000). Clathrin. Annu. Rev. Biochem. 69, 699-727.

Kjaerulff, O., Brodin, L., and Jung, A. (2011). The Structure and Function of Endophilin Proteins. Cell Biochem. Biophys. 60, 137-154.

Kloepper, T.H., Kienle, C.N., and Fasshauer, D. (2007). An Elaborate Classification of SNARE Proteins Sheds Light on the Conservation of the Eukaryotic Endomembrane System. Mol. Biol. Cell 18, 3463-3471.

Klumperman, J., and Raposo, G. (2014). The Complex Ultrastructure of the Endolysosomal System. Cold Spring Harb. Perspect. Biol. 6.

Kraemer, C., Enklaar, T., Zabel, B., and Schmidt, E.R. (2000). Mapping and structure of DMXL1, a human homologue of the DmX gene from Drosophila melanogaster coding for a WD repeat protein. Genomics 64, 97-101.

Lee, H.J., Song, J.Y., Kim, J.W., Jin, S.-Y., Hong, M.S., Park, J.K., Chung, J.-H., Shibata, H., and Fukumaki, Y. (2005). Association study of polymorphisms in synaptic vesicle-associated genes, SYN2 and CPLX2, with schizophrenia. Behav. Brain Funct. 1, 15.

Lee, S.Y., Wenk, M.R., Kim, Y., Nairn, A.C., and Camilli, P.D. (2004). Regulation of synaptojanin 1 by cyclin-dependent kinase 5 at synapses. Proc. Natl. Acad. Sci. 101, 546-551.

Leitz, J., and Kavalali, E.T. (2011). Ca ${ }^{2+}$ influx slows single synaptic vesicle endocytosis. J. Neurosci. Off. J. Soc. Neurosci. 31, 16318-16326.

Li, Z., and Murthy, V.N. (2001). Visualizing Postendocytic Traffic of Synaptic Vesicles at Hippocampal Synapses. Neuron 31, 593-605.

Li, K.W., Chen, N., Klemmer, P., Koopmans, F., Karupothula, R., and Smit, A.B. (2012). Identifying true protein complex constituents in interaction proteomics: The example of the DMXL2 protein complex. PROTEOMICS 12, 2428-2432.

Liégeois, S., Benedetto, A., Garnier, J.-M., Schwab, Y., and Labouesse, M. (2006). The V0ATPase mediates apical secretion of exosomes containing Hedgehog-related proteins in Caenorhabditis elegans. J. Cell Biol. 173, 949-961.

Lindau, M., and Alvarez de Toledo, G. (2003). The fusion pore. Biochim. Biophys. Acta 1641, 167-173. 
Littleton, J.T., Barnard, R.J.O., Titus, S.A., Slind, J., Chapman, E.R., and Ganetzky, B. (2001). SNARE-complex disassembly by NSF follows synaptic-vesicle fusion. Proc. Natl. Acad. Sci. U. S. A. $98,12233-12238$.

Loll, P.J., Swain, E., Chen, Y., Turner, B.T., and Zhang, J. (2008). Structure of the SH3 domain of rat endophilin A2. Acta Crystallograph. Sect. F Struct. Biol. Cryst. Commun. 64, 243-246.

López-Font, I., Torregrosa-Hetland, C.J., Villanueva, J., and Gutiérrez, L.M. (2010). t-SNARE cluster organization and dynamics in chromaffin cells. J. Neurochem. 114, 1550-1556.

Malacombe, M., Ceridono, M., Calco, V., Chasserot-Golaz, S., McPherson, P.S., Bader, M.-F., and Gasman, S. (2006). Intersectin-1L nucleotide exchange factor regulates secretory granule exocytosis by activating Cdc42. EMBO J. 25, 3494-3503.

Mani, M., Lee, S.Y., Lucast, L., Cremona, O., Di Paolo, G., De Camilli, P., and Ryan, T.A. (2007). The dual phosphatase activity of synaptojanin1 is required for both efficient synaptic vesicle endocytosis and reavailability at nerve terminals. Neuron 56, 1004-1018.

Marina, N., Turovsky, E., Christie, I.N., Hosford, P.S., Hadjihambi, A., Korsak, A., Ang, R., Mastitskaya, S., Sheikhbahaei, S., Theparambil, S.M., et al. (2018). Brain metabolic sensing and metabolic signaling at the level of an astrocyte. Glia 66, 1185-1199.

Maritzen, T., and Haucke, V. (2018). Coupling of exocytosis and endocytosis at the presynaptic active zone. Neurosci. Res. 127, 45-52.

Marshansky, V., Rubinstein, J.L., and Grüber, G. (2014). Eukaryotic V-ATPase: Novel structural findings and functional insights. Biochim. Biophys. Acta BBA - Bioenerg. 1837, 857-879.

Marvin, J.S., Borghuis, B.G., Tian, L., Cichon, J., Harnett, M.T., Akerboom, J., Gordus, A., Renninger, S.L., Chen, T.-W., Bargmann, C.I., et al. (2013). An optimized fluorescent probe for visualizing glutamate neurotransmission. Nat. Methods 10, 162-170.

Masuda, M., Takeda, S., Sone, M., Ohki, T., Mori, H., Kamioka, Y., and Mochizuki, N. (2006). Endophilin BAR domain drives membrane curvature by two newly identified structure-based mechanisms. EMBO J. 25, 2889-2897.

Matteoli, M., Takei, K., Cameron, R., Hurlbut, P., Johnston, P.A., Südhof, T.C., Jahn, R., and De Camilli, P. (1991). Association of Rab3A with synaptic vesicles at late stages of the secretory pathway. J. Cell Biol. 115, 625-633.

Maxson, M.E., and Grinstein, S. (2014). The vacuolar-type H+-ATPase at a glance - more than a proton pump. J Cell Sci 127, 4987-4993.

Maycox, P.R., Link, E., Reetz, A., Morris, S.A., and Jahn, R. (1992). Clathrin-coated vesicles in nervous tissue are involved primarily in synaptic vesicle recycling. J. Cell Biol. 118, 1379-1388.

McMahon, H.T., Missler, M., Li, C., and Südhof, T.C. (1995). Complexins: cytosolic proteins that regulate SNAP receptor function. Cell 83, 111-119. 
Merkulova, M., Păunescu, T.G., Azroyan, A., Marshansky, V., Breton, S., and Brown, D. (2015). Mapping the $\mathrm{H}(+)(V)$-ATPase interactome: identification of proteins involved in trafficking, folding, assembly and phosphorylation. Sci. Rep. 5, 14827.

Miesenböck, G., De Angelis, D.A., and Rothman, J.E. (1998). Visualizing secretion and synaptic transmission with $\mathrm{pH}$-sensitive green fluorescent proteins. Nature 394, 192-195.

Miller, E.W., Lin, J.Y., Frady, E.P., Steinbach, P.A., Kristan, W.B., and Tsien, R.Y. (2012). Optically monitoring voltage in neurons by photo-induced electron transfer through molecular wires. Proc. Natl. Acad. Sci. 109, 2114-2119.

Milosevic, I. (2018). Revisiting the Role of Clathrin-Mediated Endoytosis in Synaptic Vesicle Recycling. Front. Cell. Neurosci. 12.

Milosevic, I., Giovedi, S., Lou, X., Raimondi, A., Collesi, C., Shen, H., Paradise, S., O'Toole, E., Ferguson, S., Cremona, O., et al. (2011). Recruitment of Endophilin to Clathrin-Coated Pit Necks Is Required for Efficient Vesicle Uncoating after Fission. Neuron 72, 587-601.

Milovanovic, D., and De Camilli, P. (2017). Synaptic vesicle clusters at synapses: a distinct liquid phase? Neuron 93, 995-1002.

Milovanovic, D., Wu, Y., Bian, X., and Camilli, P.D. (2018). A liquid phase of synapsin and lipid vesicles. Science 361, 604-607.

Misura, K.M.S., Scheller, R.H., and Weis, W.I. (2000). Three-dimensional structure of the neuronal-Sec1-syntaxin 1a complex. Nature 404, 355-362.

Mollard, G.F. von, Südhof, T.C., and Jahn, R. (1991). A small GTP-binding protein dissociates from synaptic vesicles during exocytosis. Nature 349, 79-81.

Muench, S.P., Huss, M., Song, C.F., Phillips, C., Wieczorek, H., Trinick, J., and Harrison, M.A. (2009). Cryo-electron microscopy of the vacuolar ATPase motor reveals its mechanical and regulatory complexity. J. Mol. Biol. 386, 989-999.

Murdoch, J.D., Rostosky, C.M., Gowrisankaran, S., Arora, A.S., Soukup, S.-F., Vidal, R., Capece, V., Freytag, S., Fischer, A., Verstreken, P., et al. (2016). Endophilin-A Deficiency Induces the Foxo3a-Fbxo32 Network in the Brain and Causes Dysregulation of Autophagy and the UbiquitinProteasome System. Cell Rep. 17, 1071-1086.

Murthy, V.N., and Camilli, P.D. (2003). CELLBIOLOGY OF THE PRESYNAPTICTERMINAL. Annu. Rev. Neurosci. 26, 701-728.

Nagano, F., Kawabe, H., Nakanishi, H., Shinohara, M., Deguchi-Tawarada, M., Takeuchi, M., Sasaki, T., and Takai, Y. (2002). Rabconnectin-3, a Novel Protein That Binds Both GDP/GTP Exchange Protein and GTPase-activating Protein for Rab3 Small G Protein Family. J. Biol. Chem. 277, 9629-9632. 
Neher, E. (2006). A comparison between exocytic control mechanisms in adrenal chromaffin cells and a glutamatergic synapse. Pflüg. Arch. 453, 261-268.

Neher, E. (2010). What is Rate-Limiting during Sustained Synaptic Activity: Vesicle Supply or the Availability of Release Sites. Front. Synaptic Neurosci. 2.

Neher, E., and Sakaba, T. (2008). Multiple Roles of Calcium lons in the Regulation of Neurotransmitter Release. Neuron 59, 861-872.

Nelson, N. (2003). A journey from mammals to yeast with vacuolar H+-ATPase (V-ATPase). J. Bioenerg. Biomembr. 35, 281-289.

Nuckels, R.J., Ng, A., Darland, T., and Gross, J.M. (2009). The vacuolar-ATPase complex regulates retinoblast proliferation and survival, photoreceptor morphogenesis, and pigmentation in the zebrafish eye. Invest. Ophthalmol. Vis. Sci. 50, 893-905.

Okamoto, M., Schoch, S., and Südhof, T.C. (1999). EHSH1/intersectin, a protein that contains $\mathrm{EH}$ and $\mathrm{SH} 3$ domains and binds to dynamin and SNAP-25. A protein connection between exocytosis and endocytosis? J. Biol. Chem. 274, 18446-18454.

Onofri, F., Giovedi, S., Kao, H.T., Valtorta, F., Bongiorno Borbone, L., De Camilli, P., Greengard, P., and Benfenati, F. (2000). Specificity of the binding of synapsin I to Src homology 3 domains. J. Biol. Chem. 275, 29857-29867.

Owen, D.J., Collins, B.M., and Evans, P.R. (2004). Adaptors for clathrin coats: structure and function. Annu. Rev. Cell Dev. Biol. 20, 153-191.

Paillart, C., Li, J., Matthews, G., and Sterling, P. (2003). Endocytosis and vesicle recycling at a ribbon synapse. J. Neurosci. Off. J. Soc. Neurosci. 23, 4092-4099.

Parsons, T.D., Coorssen, J.R., Horstmann, H., and Almers, W. (1995). Docked granules, the exocytic burst, and the need for ATP hydrolysis in endocrine cells. Neuron 15, 1085-1096.

Pavlos, N.J., and Jahn, R. (2011). Distinct yet overlapping roles of Rab GTPases on synaptic vesicles. Small GTPases 2, 77-81.

Pechstein, A., and Shupliakov, O. (2010). Taking a back seat: synaptic vesicle clustering in presynaptic terminals. Front. Synaptic Neurosci. 2, 143.

Pechstein, A., Bacetic, J., Vahedi-Faridi, A., Gromova, K., Sundborger, A., Tomlin, N., Krainer, G., Vorontsova, O., Schäfer, J.G., Owe, S.G., et al. (2010). Regulation of synaptic vesicle recycling by complex formation between intersectin 1 and the clathrin adaptor complex AP2. Proc. Natl. Acad. Sci. 107, 4206-4211.

Pechstein, A., Gerth, F., Milosevic, I., Jäpel, M., Eichhorn-Grünig, M., Vorontsova, O., Bacetic, J., Maritzen, T., Shupliakov, O., Freund, C., et al. (2015). Vesicle uncoating regulated by SH3-SH3 domain-mediated complex formation between endophilin and intersectin at synapses. EMBO Rep. 16, 232-239. 
Perera, R.M., and Zoncu, R. (2016). The Lysosome as a Regulatory Hub. Annu. Rev. Cell Dev. Biol. 32, 223-253.

Perera, R.M., Zoncu, R., Lucast, L., Camilli, P.D., and Toomre, D. (2006). Two synaptojanin 1 isoforms are recruited to clathrin-coated pits at different stages. Proc. Natl. Acad. Sci. 103, $19332-19337$.

Perin, M.S., Fried, V.A., Mignery, G.A., Jahn, R., and Südhof, T.C. (1990). Phospholipid binding by a synaptic vesicle protein homologous to the regulatory region of protein kinase C. Nature 345 , 260-263.

Peters, C., Bayer, M.J., Bühler, S., Andersen, J.S., Mann, M., and Mayer, A. (2001). Transcomplex formation by proteolipid channels in the terminal phase of membrane fusion. Nature 409, 581-588.

Pinheiro, P.S., Jansen, A.M., de Wit, H., Tawfik, B., Madsen, K.L., Verhage, M., Gether, U., and Sørensen, J.B. (2014). The BAR domain protein PICK1 controls vesicle number and size in adrenal chromaffin cells. J. Neurosci. Off. J. Soc. Neurosci. 34, 10688-10700.

Plattner, H., Artalejo, A.R., and Neher, E. (1997). Ultrastructural Organization of Bovine Chromaffin Cell Cortex-Analysis by Cryofixation and Morphometry of Aspects Pertinent to Exocytosis. J. Cell Biol. 139, 1709-1717.

Poëa-Guyon, S., Ammar, M.R., Erard, M., Amar, M., Moreau, A.W., Fossier, P., Gleize, V., Vitale, N., and Morel, N. (2013). The V-ATPase membrane domain is a sensor of granular $\mathrm{pH}$ that controls the exocytotic machinery. J. Cell Biol. 203, 283-298.

Raimondi, A., Ferguson, S.M., Lou, X., Armbruster, M., Paradise, S., Giovedi, S., Messa, M., Kono, N., Takasaki, J., Cappello, V., et al. (2011). Overlapping Role of Dynamin Isoforms in Synaptic Vesicle Endocytosis. Neuron 70, 1100-1114.

Rangaraju, V., Calloway, N., and Ryan, T.A. (2014). Activity-Driven Local ATP Synthesis Is Required for Synaptic Function. Cell 156, 825-835.

Renard, H.-F., Simunovic, M., Lemière, J., Boucrot, E., Garcia-Castillo, M.D., Arumugam, S., Chambon, V., Lamaze, C., Wunder, C., Kenworthy, A.K., et al. (2015). Endophilin-A2 functions in membrane scission in clathrin-independent endocytosis. Nature 517, 493-496.

Rettig, J., and Neher, E. (2002). Emerging roles of presynaptic proteins in Ca++-triggered exocytosis. Science 298, 781-785.

Revelo, N.H., Kamin, D., Truckenbrodt, S., Wong, A.B., Reuter-Jessen, K., Reisinger, E., Moser, T., and Rizzoli, S.O. (2014). A new probe for super-resolution imaging of membranes elucidates trafficking pathways. J Cell Biol 205, 591-606.

Ringstad, N., Nemoto, Y., and De Camilli, P. (1997). The SH3p4/Sh3p8/SH3p13 protein family: Binding partners for synaptojanin and dynamin via a Grb2-like Src homology 3 domain. Proc. Natl. Acad. Sci. 94, 8569-8574. 
Ringstad, N., Gad, H., Löw, P., Di Paolo, G., Brodin, L., Shupliakov, O., and De Camilli, P. (1999). Endophilin/SH3p4 is required for the transition from early to late stages in clathrin-mediated synaptic vesicle endocytosis. Neuron 24, 143-154.

Rizzoli, S.O. (2014). Synaptic vesicle recycling: steps and principles. EMBO J. 33, 788-822.

Rizzoli, S.O., and Betz, W.J. (2005). Synaptic vesicle pools. Nat. Rev. Neurosci. 6, 57-69.

Rizzoli, S.O., Bethani, I., Zwilling, D., Wenzel, D., Siddiqui, T.J., Brandhorst, D., and Jahn, R. (2006). Evidence for early endosome-like fusion of recently endocytosed synaptic vesicles. Traffic Cph. Den. 7, 1163-1176.

Rosenmund, C., and Stevens, C.F. (1996). Definition of the readily releasable pool of vesicles at hippocampal synapses. Neuron 16, 1197-1207.

Rost, B.R., Schneider, F., Grauel, M.K., Wozny, C., Bentz, C., Blessing, A., Rosenmund, T., Jentsch, T.J., Schmitz, D., Hegemann, P., et al. (2015). Optogenetic acidification of synaptic vesicles and lysosomes. Nat. Neurosci. 18, 1845-1852.

Saheki, Y., and De Camilli, P. (2012). Synaptic vesicle endocytosis. Cold Spring Harb. Perspect. Biol. 4, a005645.

Sakaba, T., Kononenko, N.L., Bacetic, J., Pechstein, A., Schmoranzer, J., Yao, L., Barth, H., Shupliakov, O., Kobler, O., Aktories, K., et al. (2013). Fast neurotransmitter release regulated by the endocytic scaffold intersectin. Proc. Natl. Acad. Sci. U. S. A. 110, 8266-8271.

Sankaranarayanan, S., and Ryan, T.A. (2000). Real-time measurements of vesicle-SNARE recycling in synapses of the central nervous system. Nat. Cell Biol. 2, 197-204.

Sankaranarayanan, R., Nene, B.M., Dinshaw, K., Rajkumar, R., Shastri, S., Wesley, R., Basu, P., Sharma, R., Thara, S., Budukh, A., et al. (2003). Early detection of cervical cancer with visual inspection methods: a summary of completed and on-going studies in India. Salud Publica Mex. 45 Suppl 3, S399-407.

Sankaranarayanan, S., De Angelis, D., Rothman, J.E., and Ryan, T.A. (2000). The use of pHluorins for optical measurements of presynaptic activity. Biophys. J. 79, 2199-2208.

Sautin, Y.Y., Lu, M., Gaugler, A., Zhang, L., and Gluck, S.L. (2005). Phosphatidylinositol 3-kinasemediated effects of glucose on vacuolar $\mathrm{H}+$-ATPase assembly, translocation, and acidification of intracellular compartments in renal epithelial cells. Mol. Cell. Biol. 25, 575-589.

Schuske, K.R., Richmond, J.E., Matthies, D.S., Davis, W.S., Runz, S., Rube, D.A., van der Bliek, A.M., and Jorgensen, E.M. (2003). Endophilin Is Required for Synaptic Vesicle Endocytosis by Localizing Synaptojanin. Neuron 40, 749-762.

Seal, R.P., Akil, O., Yi, E., Weber, C.M., Grant, L., Yoo, J., Clause, A., Kandler, K., Noebels, J.L., Glowatzki, E., et al. (2008). Sensorineural Deafness and Seizures in Mice Lacking Vesicular Glutamate Transporter 3. Neuron 57, 263-275. 
Seol, J.H., Shevchenko, A., Shevchenko, A., and Deshaies, R.J. (2001). Skp1 forms multiple protein complexes, including RAVE, a regulator of V-ATPase assembly. Nat. Cell Biol. 3, 384391.

Sethi, N., Yan, Y., Quek, D., Schupbach, T., and Kang, Y. (2010). Rabconnectin-3 Is a Functional Regulator of Mammalian Notch Signaling. J. Biol. Chem. 285, 34757-34764.

Shin, W., Ge, L., Arpino, G., Villarreal, S.A., Hamid, E., Liu, H., Zhao, W.-D., Wen, P.J., Chiang, H.-C., and Wu, L.-G. (2018). Visualization of Membrane Pore in Live Cells Reveals a DynamicPore Theory Governing Fusion and Endocytosis. Cell 173, 934-945.e12.

Shupliakov, O. (2009). The synaptic vesicle cluster: a source of endocytic proteins during neurotransmitter release. Neuroscience 158, 204-210.

Shupliakov, O., Haucke, V., and Pechstein, A. (2011). How synapsin I may cluster synaptic vesicles. Semin. Cell Dev. Biol. 22, 393-399.

Sipos, G., Brickner, J.H., Brace, E. j., Chen, L., Rambourg, A., Kepes, F., and Fuller, R.S. (2004). Soi3p/Rav1p Functions at the Early Endosome to Regulate Endocytic Trafficking to the Vacuole and Localization of Trans-Golgi Network Transmembrane Proteins. Mol. Biol. Cell 15, 3196-3209.

Smardon, A.M., and Kane, P.M. (2007). RAVE Is Essential for the Efficient Assembly of the C Subunit with the Vacuolar H+-ATPase. J. Biol. Chem. 282, 26185-26194.

Smardon, A.M., Tarsio, M., and Kane, P.M. (2002). The RAVE Complex Is Essential for Stable Assembly of the Yeast V-ATPase. J. Biol. Chem. 277, 13831-13839.

Smith, C., and Neher, E. (1997). Multiple Forms of Endocytosis In Bovine Adrenal Chromaffin Cells. J. Cell Biol. 139, 885-894.

Smith, S.M., Renden, R., and von Gersdorff, H. (2008). Synaptic vesicle endocytosis: fast and slow modes of membrane retrieval. Trends Neurosci. 31, 559-568.

So, C.W., Sham, M.H., Chew, S.L., Cheung, N., So, C.K., Chung, S.K., Caldas, C., Wiedemann, L.M., and Chan, L.C. (2000). Expression and protein-binding studies of the EEN gene family, new interacting partners for dynamin, synaptojanin and huntingtin proteins. Biochem. J. 348 Pt 2, 447458.

Söllner, T., Whiteheart, S.W., Brunner, M., Erdjument-Bromage, H., Geromanos, S., Tempst, P., and Rothman, J.E. (1993). SNAP receptors implicated in vesicle targeting and fusion. Nature 362, 318-324.

Sørensen, J.B. (2004). Formation, stabilisation and fusion of the readily releasable pool of secretory vesicles. Pflüg. Arch. 448, 347-362.

Sørensen, J.B., Nagy, G., Varoqueaux, F., Nehring, R.B., Brose, N., Wilson, M.C., and Neher, E. (2003). Differential Control of the Releasable Vesicle Pools by SNAP-25 Splice Variants and SNAP-23. Cell 114, 75-86. 
Soukup, S.-F., and Verstreken, P. (2017). EndoA/Endophilin-A creates docking stations for autophagic proteins at synapses. Autophagy 13, 971-972.

Soykan, T., Maritzen, T., and Haucke, V. (2016). Modes and mechanisms of synaptic vesicle recycling. Curr. Opin. Neurobiol. 39, 17-23.

Soykan, T., Kaempf, N., Sakaba, T., Vollweiter, D., Goerdeler, F., Puchkov, D., Kononenko, N.L., and Haucke, V. (2017). Synaptic Vesicle Endocytosis Occurs on Multiple Timescales and Is Mediated by Formin-Dependent Actin Assembly. Neuron 93, 854-866.e4.

Stein, A., Weber, G., Wahl, M.C., and Jahn, R. (2009). Helical extension of the neuronal SNARE complex into the membrane. Nature 460, 525-528.

Strasser, B., Iwaszkiewicz, J., Michielin, O., and Mayer, A. (2011). The V-ATPase proteolipid cylinder promotes the lipid-mixing stage of SNARE-dependent fusion of yeast vacuoles. EMBO J. 30, 4126-4141.

Südhof, T.C. (2012). The presynaptic active zone. Neuron 75, 11-25.

Südhof, T.C. (2013). Neurotransmitter release: the last millisecond in the life of a synaptic vesicle. Neuron 80, 675-690.

Südhof, T.C., and Rizo, J. (2011). Synaptic vesicle exocytosis. Cold Spring Harb. Perspect. Biol. 3.

Südhof, T.C., and Rothman, J.E. (2009). Membrane fusion: grappling with SNARE and SM proteins. Science 323, 474-477.

Sumner, J.P., Dow, J.A., Earley, F.G., Klein, U., Jäger, D., and Wieczorek, H. (1995). Regulation of plasma membrane V-ATPase activity by dissociation of peripheral subunits. J. Biol. Chem. 270, 5649-5653.

Tagliatti, E., Fadda, M., Falace, A., Benfenati, F., and Fassio, A. (2016). Arf6 regulates the cycling and the readily releasable pool of synaptic vesicles at hippocampal synapse.

Takamori, S., Rhee, J.S., Rosenmund, C., and Jahn, R. (2000). Identification of a vesicular glutamate transporter that defines a glutamatergic phenotype in neurons. Nature 407, 189-194.

Takamori, S., Holt, M., Stenius, K., Lemke, E.A., Grønborg, M., Riedel, D., Urlaub, H., Schenck, S., Brügger, B., Ringler, P., et al. (2006). Molecular anatomy of a trafficking organelle. Cell 127, 831-846.

Takei, K. (1996). The synaptic vesicle cycle: a single vesicle budding step involving clathrin and dynamin. J. Cell Biol. 133, 1237-1250.

Tao-Cheng, J.-H. (2006). Activity-related redistribution of presynaptic proteins at the active zone. Neuroscience 141, 1217-1224. 
Tata, B., Huijbregts, L., Jacquier, S., Csaba, Z., Genin, E., Meyer, V., Leka, S., Dupont, J., Charles, P., Chevenne, D., et al. (2014). Haploinsufficiency of Dmxl2, Encoding a Synaptic Protein, Causes Infertility Associated with a Loss of GnRH Neurons in Mouse. PLoS Biol. 12.

Tata, B.K., Harbulot, C., Csaba, Z., Peineau, S., Jacquier, S., and Roux, N. de (2017). Rabconnectin-3 $\alpha$ is required for the morphological maturation of $\mathrm{GnRH}$ neurons and kisspeptin responsiveness. Sci. Rep. 7, 42463.

Toei, M., Saum, R., and Forgac, M. (2010). Regulation and isoform function of the V-ATPases. Biochemistry 49, 4715-4723.

Trempe, J.-F., Chen, C.X.-Q., Grenier, K., Camacho, E.M., Kozlov, G., McPherson, P.S., Gehring, K., and Fon, E.A. (2009). SH3 Domains from a Subset of BAR Proteins Define a Ubl-Binding Domain and Implicate Parkin in Synaptic Ubiquitination. Mol. Cell 36, 1034-1047.

Trimbuch, T., and Rosenmund, C. (2016). Should I stop or should I go? The role of complexin in neurotransmitter release. Nat. Rev. Neurosci. 17, 118-125.

Tuttle, A.M., Hoffman, T.L., and Schilling, T.F. (2014). Rabconnectin-3a regulates vesicle endocytosis and canonical Wnt signaling in zebrafish neural crest migration. PLoS Biol. 12, e1001852.

Uytterhoeven, V., Lauwers, E., Maes, I., Miskiewicz, K., Melo, M.N., Swerts, J., Kuenen, S., Wittocx, R., Corthout, N., Marrink, S.-J., et al. (2015). Hsc70-4 Deforms Membranes to Promote Synaptic Protein Turnover by Endosomal Microautophagy. Neuron 88, 735-748.

Van Dyke, R.W., and Belcher, J.D. (1994). Acidification of three types of liver endocytic vesicles: similarities and differences. Am. J. Physiol. 266, C81-94.

Vardar, G., Chang, S., Arancillo, M., Wu, Y.-J., Trimbuch, T., and Rosenmund, C. (2016). Distinct Functions of Syntaxin-1 in Neuronal Maintenance, Synaptic Vesicle Docking, and Fusion in Mouse Neurons. J. Neurosci. 36, 7911-7924.

Vargas, K.J., Schrod, N., Davis, T., Fernandez-Busnadiego, R., Taguchi, Y.V., Laugks, U., Lucic, V., and Chandra, S.S. (2017). Synucleins Have Multiple Effects on Presynaptic Architecture. Cell Rep. 18, 161-173.

Veit, M., Söllner, T.H., and Rothman, J.E. (1996). Multiple palmitoylation of synaptotagmin and the t-SNARE SNAP-25. FEBS Lett. 385, 119-123.

Verhage, M., and Sørensen, J.B. (2008). Vesicle Docking in Regulated Exocytosis. Traffic 9, 1414-1424.

Verhage, M., Maia, A.S., Plomp, J.J., Brussaard, A.B., Heeroma, J.H., Vermeer, H., Toonen, R.F., Hammer, R.E., van den Berg, T.K., Missler, M., et al. (2000). Synaptic assembly of the brain in the absence of neurotransmitter secretion. Science 287, 864-869. 
Verstreken, P., Koh, T.-W., Schulze, K.L., Zhai, R.G., Hiesinger, P.R., Zhou, Y., Mehta, S.Q., Cao, Y., Roos, J., and Bellen, H.J. (2003). Synaptojanin Is Recruited by Endophilin to Promote Synaptic Vesicle Uncoating. Neuron 40, 733-748.

Vinatier, J., Herzog, E., Plamont, M.-A., Wojcik, S.M., Schmidt, A., Brose, N., Daviet, L., El Mestikawy, S., and Giros, B. (2006). Interaction between the vesicular glutamate transporter type 1 and endophilin A1, a protein essential for endocytosis. J. Neurochem. 97, 1111-1125.

Vitavska, O., Merzendorfer, H., and Wieczorek, H. (2005). The V-ATPase subunit C binds to polymeric $\mathrm{F}$-actin as well as to monomeric $\mathrm{G}$-actin and induces cross-linking of actin filaments. J. Biol. Chem. 280, 1070-1076.

Voets, T. (2000). Dissection of three Ca2+-dependent steps leading to secretion in chromaffin cells from mouse adrenal slices. Neuron 28, 537-545.

Voets, T., Neher, E., and Moser, T. (1999). Mechanisms Underlying Phasic and Sustained Secretion in Chromaffin Cells from Mouse Adrenal Slices. Neuron 23, 607-615.

Wandinger-Ness, A., and Zerial, M. (2014). Rab proteins and the compartmentalization of the endosomal system. Cold Spring Harb. Perspect. Biol. 6, a022616.

Wang, Y., and Südhof, T.C. (2003). Genomic definition of RIM proteins: evolutionary amplification of a family of synaptic regulatory proteins. Genomics 81, 126-137.

Wang, Y., Okamoto, M., Schmitz, F., Hofmann, K., and Südhof, T.C. (1997). Rim is a putative Rab3 effector in regulating synaptic-vesicle fusion. Nature 388, 593-598.

Watanabe, S., and Boucrot, E. (2017). Fast and ultrafast endocytosis. Curr. Opin. Cell Biol. 47, 64-71.

Watanabe, S., Liu, Q., Davis, M.W., Hollopeter, G., Thomas, N., Jorgensen, N.B., and Jorgensen, E.M. (2013a). Ultrafast endocytosis at Caenorhabditis elegans neuromuscular junctions. ELife 2, e00723.

Watanabe, S., Rost, B.R., Camacho-Pérez, M., Davis, M.W., Söhl-Kielczynski, B., Rosenmund, C., and Jorgensen, E.M. (2013b). Ultrafast endocytosis at mouse hippocampal synapses. Nature 504, 242-247.

Watanabe, S., Trimbuch, T., Camacho-Pérez, M., Rost, B.R., Brokowski, B., Söhl-Kielczynski, B., Felies, A., Davis, M.W., Rosenmund, C., and Jorgensen, E.M. (2014). Clathrin regenerates synaptic vesicles from endosomes. Nature 515, 228-233.

Watanabe, S., Mamer, L.E., Raychaudhuri, S., Luvsanjav, D., Eisen, J., Trimbuch, T., SöhlKielczynski, B., Fenske, P., Milosevic, I., Rosenmund, C., et al. (2018). Synaptojanin and Endophilin Mediate Neck Formation during Ultrafast Endocytosis. Neuron 98, 1184-1197.e6. 
Weber, T., Zemelman, B.V., McNew, J.A., Westermann, B., Gmachl, M., Parlati, F., Söllner, T.H., and Rothman, J.E. (1998). SNAREpins: minimal machinery for membrane fusion. Cell 92, 759772.

Weston, M.C., Nehring, R.B., Wojcik, S.M., and Rosenmund, C. (2011). Interplay between VGLUT Isoforms and Endophilin A1 Regulates Neurotransmitter Release and Short-Term Plasticity. Neuron 69, 1147-1159.

Wojcik, S.M., and Brose, N. (2007). Regulation of membrane fusion in synaptic excitationsecretion coupling: speed and accuracy matter. Neuron 55, 11-24.

Wojcik, S.M., Rhee, J.S., Herzog, E., Sigler, A., Jahn, R., Takamori, S., Brose, N., and Rosenmund, C. (2004). An essential role for vesicular glutamate transporter 1 (VGLUT1) in postnatal development and control of quantal size. Proc. Natl. Acad. Sci. U. S. A. 101, 71587163.

Wu, W., and Wu, L.-G. (2007). Rapid bulk endocytosis and its kinetics of fission pore closure at a central synapse. Proc. Natl. Acad. Sci. U. S. A. 104, 10234-10239.

Wu, X.-S., McNeil, B.D., Xu, J., Fan, J., Xue, L., Melicoff, E., Adachi, R., Bai, L., and Wu, L.-G. (2009). $\mathrm{Ca}(2+)$ and calmodulin initiate all forms of endocytosis during depolarization at a nerve terminal. Nat. Neurosci. 12, 1003-1010.

Xing, Y., Böcking, T., Wolf, M., Grigorieff, N., Kirchhausen, T., and Harrison, S.C. (2010). Structure of clathrin coat with bound Hsc70 and auxilin: mechanism of Hsc70-facilitated disassembly. EMBO J. 29, 655-665.

Yan, Y., Denef, N., and Schüpbach, T. (2009). The vacuolar proton pump (V-ATPase) is required for Notch signaling and endosomal trafficking in Drosophila. Dev. Cell 17, 387-402.

Zerial, M., and McBride, H. (2001). Rab proteins as membrane organizers. Nat. Rev. Mol. Cell Biol. 2, 107-117.

Zhang, Q., Li, Y., and Tsien, R.W. (2009). The dynamic control of kiss-and-run and vesicular reuse probed with single nanoparticles. Science 323, 1448-1453.

Zhao, J., Benlekbir, S., and Rubinstein, J.L. (2015). Electron cryomicroscopy observation of rotational states in a eukaryotic V-ATPase. Nature 521, 241-245.

Zhou, Q., Petersen, C.C., and Nicoll, R.A. (2000). Effects of reduced vesicular filling on synaptic transmission in rat hippocampal neurones. J. Physiol. 525 Pt 1, 195-206.

Zoncu, R., Bar-Peled, L., Efeyan, A., Wang, S., Sancak, Y., and Sabatini, D.M. (2011). mTORC1 Senses Lysosomal Amino Acids Through an Inside-Out Mechanism That Requires the Vacuolar H+-ATPase. Science 334, 678-683. 


\section{Acknowledgements}

I would like to express my sincere gratitude and thanks to all the people who have been the pillars of support through the years of my PhD thesis.

Dr. Ira Milosevic, for providing me the opportunity to pursue PhD thesis, her supervision and sharing the enthusiasm for science. It was a great learning experience. I am thankful for the freedom I had to explore many techniques and try new things consistently; I thoroughly enjoyed pursuing my projects and hope to have learnt something from this.

My thesis committee members Prof. Reinhard Jahn and Prof. Nils Brose. It was inspiring to present my progress work to the committee and the feedbacks were very crucial for this thesis work. I am also thankful for their support to use resources from their respective departments and perform experiments there. 
Prof. Jakob Sorensen, for providing the chance to learn electrophysiology and sharing his passion for work. I learnt a lot about work ethics in my 3 months at Copenhagen. I am also thankful to all the lab members of his lab, especially Sebastien, Bassam, Melanie and Anne-Marie for making it a productive and memorable time.

Dr. Nuno Raimundo, who provided great motivation, constant support, all the help and nice discussion in the lab meetings. I hope to learn more about cellular organelle signaling someday.

All the former and current lab members of the Milosevic lab and Raimundo lab. I enjoyed sharing the office with llona, Lupita and Anita, for all the stupidity, laughter and of course the scientific discussions. You guys made my second home comfortable. I would like to extend my sincere thanks to Christine for the help with mouse work, King for all the help with experiments and trouble shooting discussions and Dr.Krisko for the motivation. Also, thank you so much for reading my thesis chapters and support during the last phase of the PhD. A special mention about Melanie König and Dirk Schwitters, our former and current lab technician for their awesome support during the years. I specially enjoyed the company and support of lab members Angela, Sanda, Marina, King, Lorena, Leo, Renata, Kasia, Vicky, Sofia, Tiago, Jana, Aman, Udhay and all the former members of both the labs. I would like to mention the help from Zohreh, am grateful to her for sharing her knowledge and always answering my questions. Also, am grateful for the help from friends in Jahn department during my prep days. Thanks to Ankit, Bassam and Brett Carter for the help with ephys set-up.

I am extremely grateful to the Neuroscience Program, co-ordinators Prof Micheal Hörner, Sandra Drube and Franziska for making the last 6 years in Germany such a pleasant experience and being there always when needed. I would also like to thank the support staff at ENI for the administrative support, IT team, Nils, workshop team and the animial facility staffs (also my mice colony) for enabling the research.

I am grateful to have met some awesome people through the IMPRS Master's program and their friendship made Goettingen my home in the last few years. I discovered my ramuness with Pratibha, Shrutee, Luis and Özge, during the times in the student dorm and after that, for which a simple thank you will not be enough. I enjoyed the company of Julio, Sharlen, Diego, Luis, Özge, Mari, Aarti, Sabitha, Sneha, Anika, Lina and all the others, thanks for the great memories and friendship. Thanks to the neuroball teammates as well for all the fun we had playing volleyball. Also, a big thanks to all the friends back home and in Wageningen who have been supportive through the years. It was such a pleasure to have met all these people in and outside of the lab and I will cherish the friendship always.

Lastly, I would like to thank my family, who made all of this possible because of their constant support and love and I dedicate this thesis to them. Thank you Amma and Appa for believing in me and supporting me always and grand parents for the love and care. Girish for being the best brother and looking after me, Bhargavi for being such a great friend and chinmin for bringing so much joy in all our lives. Thanks Girish and Bee for reading my thesis. I am very grateful for having such an awesome bunch of cousins who made life so much fun (you guys are the best). I made through this PhD because of you Balaji, I can't thank you enough for everything you have done so far and being so patient with me, I am looking forward to our life together. Finally, I would like to conclude my acknowledgements with the two lines which carried me forward all these years:

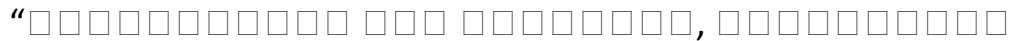


(Even if fate or God doesn't aid, perseverance will pay the wages for one's efforts) 